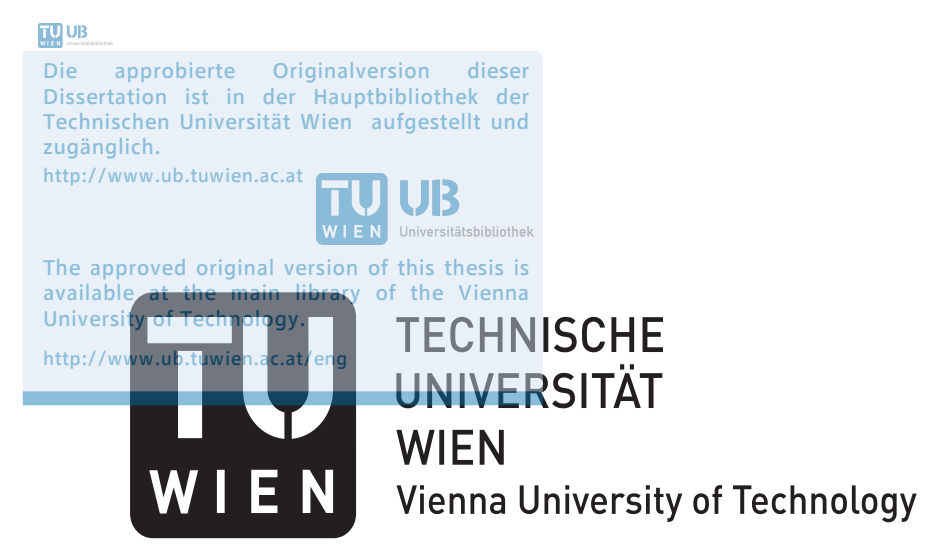

\title{
Dissertation
}

\section{Energy optimal control of thermal comfort in trams}

carried out for the purpose of obtaining the degree of Doctor technicae (Dr. techn.), submitted at TU Wien, Faculty of Mechanical and Industrial Engineering, by

\section{Raphael N. HOFSTÄDTER}

Mat. Nr.: 0525010

\author{
under the supervision of \\ Ao. Prof. Dr. DI Martin KOZEK \\ Institute of Mechanics and Mechatronics
}

reviewed by

Univ. Prof. Dr. DI Stefan JAKUBEK

Vienna University of Technology

Institute of Mechanics and Mechatronics

Getreidemarkt 9/325, 1060 Vienna, Austria
Prof. Dr. Jorge AMAYA

University of Chile

Center for Mathematical Modeling

Av. Beauchef 851, North Building,

Santiago, Chile 
I confirm, that going to press of this thesis needs the confirmation of the examination committee.

\section{Affidavit}

I declare in lieu of oath, that I wrote this thesis and performed the associated research myself, using only literature cited in this volume. If text passages from sources are used literally, they are marked as such. I confirm that this work is original and has not been submitted elsewhere for any examination, nor is it currently under consideration for a thesis elsewhere.

Salzgitter, September 2018

R.N. Hofstädter 


\section{Summary}

In this thesis, a thermal comfort control for trams is developed, which minimises the power consumption of the heating, air conditioning and ventilation system. The objective of this thesis is to achieve a satisfying indoor climate in a tram with the smallest electrical effort possible, by using methods of control engineering and closely related sciences.

In this work, mathematical models - based on the fundamental laws and balance laws of thermodynamics - of all thermally relevant parts are stated. Individual parts modelled are, the interior of the tram, the heating, ventilation and air conditioning system (HVAC), and the controller to calculate the temperature, relative humidity, and carbon dioxide concentration in the interior of the tram. For the model of the interior, a macroscopic modelling approach is chosen. For the HVAC two different approaches will be presented: The first approach is an analytical modelling of the subsystems (mixing chamber, cooling cycle and ventilators etc.) to depict the entire system. The model of the entire system is non-linear. For the second approach, various simplifications are used during the modelling to gain a linearised model. The controller will be set up as a finite state machine.

In the next step, the numerical values of the parameters for the created models will be estimated. For the interior model of the tram, parameters are determined by a least squares approach from measurement data. Necessary data are gained from experiments carried out in the climatic wind tunnel (CWT). If expert knowledge about the parameters is available, it will be used as a basis for further adaptation. In the Heating, Ventilation and Air-Condition Unit, the parameters will be derived from design sketches and similar documents. If there is no or too little information, parameters will be estimated by grey-box approaches. For the controller model, the states and transition conditions of the finite state machine will be made available by the manufacturer. The estimated parameters will be validated by data, which will be collected during CWT and on-site experiments. From literature, a common model of thermal comfort is taken and numerically linearised.

With the linearised models of the plant (interior of the tram and HVAC unit) cascaded control loops are established. The master control loop, whose controller is a model-predictive controller, regulates the thermal comfort of the tram. The controller of the slave control loop will be realised with a mixed-integer optimisation, which converts the set point of the auxiliary control variable in an energy-optimal way. For the mixed-integer problem, a heuristic solution is used.

For confirming the solution approach, the algorithms were implemented on a rapid controller prototyping platform. First, the control concept was tested during several CWT tests. The annual energy consumption was extrapolated using CWT measurements. 


\section{Kurzfassung}

In dieser Dissertation wird eine Regelung des thermischen Komforts für Straßenbahnen entwickelt, welche die Leistungsaufnahme der Heizungs-, Klima- und Lüftungsanlage (HKL) minimiert. Das Ziel dieser Arbeit ist es, ein befriedigendes Innenraumklima in einer Straßenbahn mit der kleinstmöglichen elektrischen Energie zu erreichen, in dem Methoden der Regelungstechnik und eng verwandten Wissenschaften angewendet werden.

In der Arbeit werden mathematische Modelle - die auf den Grundgesetzen und Bilanzgleichungen der Thermodynamik beruhen - aller thermisch relevanten Komponenten aufgestellt. Als einzelne Komponenten werden modelliert: der Innenraum der Straßenbahn, die HKL und der Regler, um die Temperatur, relative Luftfeuchtigkeit und die Kohlenstoffdioxidkonzentration im Inneren berechnen zu können. Für das Modell des Innenraums wird ein makroskopischer Modellansatz gewählt. Für die HKL werden zwei verschiedene Ansätze vorgestellt: Beim ersten Ansatz wird durch analytische Modellierung der Subsysteme (Mischkammer, Kältekreislauf und Ventilatoren etc.) das Gesamtsystem dargestellt. Das Modell des Gesamtsystems ist nicht-linear. Für den zweiten Ansatz werden mehrere Vereinfachungen bei der Modellierung verwendet um ein linearisiertes Modell zu erhalten. Der Regler wird als endlicher Zustandsautomat abgebildet.

Im nächsten Schritt werden für die erstellten Modelle die Zahlenwerte der Parameter bestimmt. Für das Innenraummodell der Straßenbahn werden die Parameter durch einen Least-Squares Ansatz aus Messdaten bestimmt, welche aus im Klima-Wind-Kanal (KWK) durchgeführten Versuchen gewonnen werden. Ist Expertenwissen über die Parameter vorhanden, so wird es als Basis für weitere Anpassungen verwendet. Bei der HKL werden die Parameter aus Konstruktionsskizzen oder ähnlichen Unterlagen abgeleitet. Gibt es keine oder zu wenige Informationen, so werden die Parameter durch Grey-Box Ansätze geschätzt. Für das Reglermodell werden die Zustände und Übergangsbedingungen des endlichen Zustandsautomaten durch den Hersteller zugänglich gemacht. Die bestimmten Parameter werden anhand von Daten validiert, welche im KWK und auf der Strecke aufgezeichnet werden. Aus der Literatur wird ein gängiges Modell des thermischen Komforts entnommen und numerisch linearisiert.

Mit den linearisierten Modellen der Strecke (des Innenraums der Straßenbahn und der HKL Anlage) werden ineinandergeschachtelte Regelkreise eingesetzt. Der Führungsregelkreis, dessen Regler als modelprädiktiver Regler ausgeführt ist, regelt den thermischen Komfort der Straßenbahn. Der Regler des Folgeregelkreis wird durch eine gemischt ganzzahlige Optimierung realisiert, welche den Sollwert der Hilfsregelgröße energie-optimal umsetzt. Für das gemischt ganzzahlige Problem wird eine Lösungsheuristik verwendet.

Für die Validierung des Lösungsansatzes wurden die Algorithmen auf einer Rapid Controller Prototyping Platform implementiert. Das Regelungskonzept wurde zuerst in mehreren Versuchen im KWK getestet. Der Jahresenergieverbrauch wurden anhand der KWK Messungen hochgerechnet. 


\section{contents}

$\begin{array}{ll}\text { Summary } & \text { iii }\end{array}$

$\begin{array}{ll}\text { Kurzfassung } & \text { iv }\end{array}$

$\begin{array}{ll}\text { 1. Introduction } & 1\end{array}$

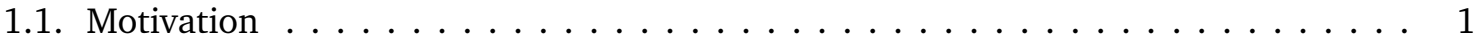

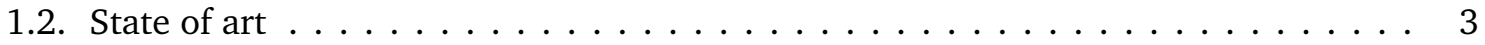

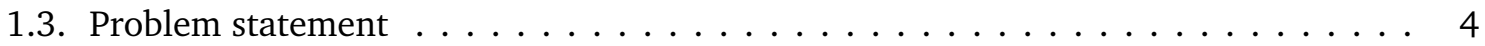

2. Fundamentals $\quad \mathbf{5}$

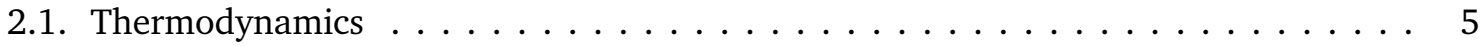

2.1.1. Laws of thermodynamics ................... 5

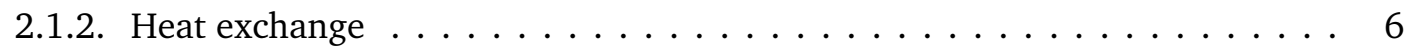

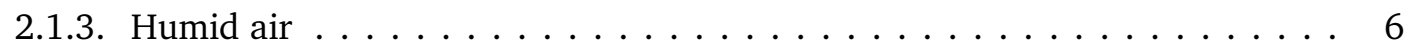

2.1.4. Heating, ventilation and air conditioning system . . . . . . . . . . 8

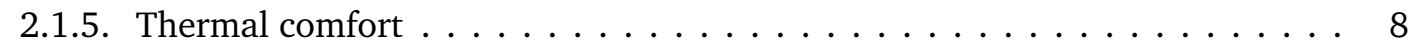

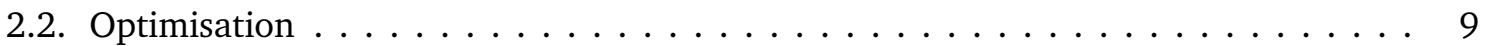

2.2.1. Convex optimisation . . . . . . . . . . . . . . . . . 9

2.2.2. Mixed integer optimisation . . . . . . . . . . . . . . . . . . . . . . . . . . . .

2.2.3. Multi-criteria optimisation . . . . . . . . . . . . . . . 10

2.3. Control engineering . . . . . . . . . . . . . . . . . . . . 11

2.3.1. General state space system . . . . . . . . . . . . . . . . . . . . . . . . . . . . . . . . .

2.3.2. Cascaded control . . . . . . . . . . . . . . . . . . . 12

2.3.3. Model predictive control . . . . . . . . . . . . . . . . . 12

3. Modelling $r$

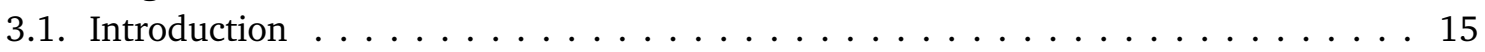

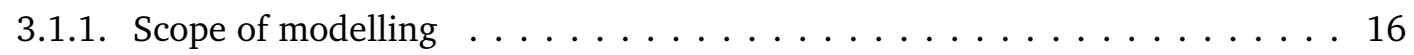

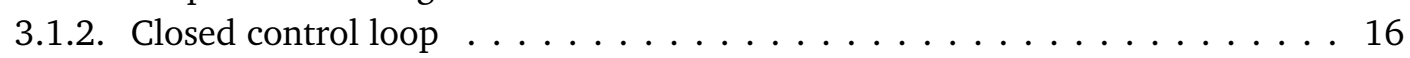

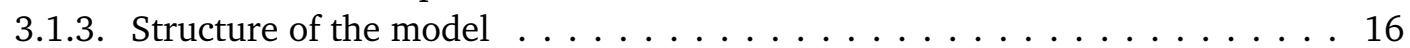

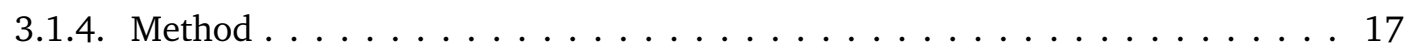

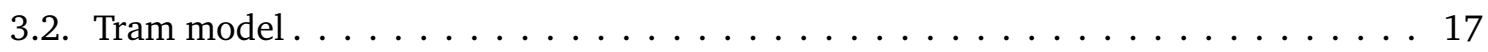

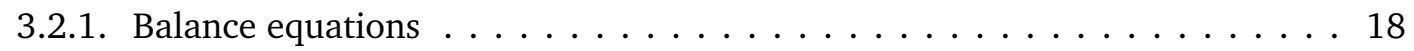

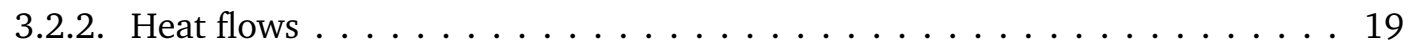

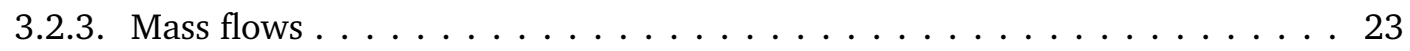

3.3. HVAC model . . . . . . . . . . . . . . . . . . . . . . . 24

3.3.1. Electric heater . . . . . . . . . . . . . . . . . 25

3.3.2. Compression refrigeration machine ................ 26

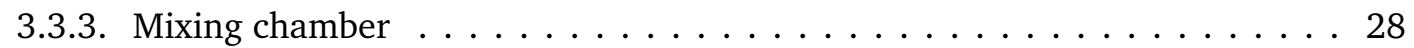


3.3.4. Fans . . . . . . . . . . . . . . . . . . . . . . . . . . . . 29

3.4. Controller model of the current controller . . . . . . . . . . . . . . . . . 29

3.4.1. Global finite state machine $(\mathrm{FSM}) \ldots \ldots \ldots$. . . . . . . . . . . . . . . . . 30

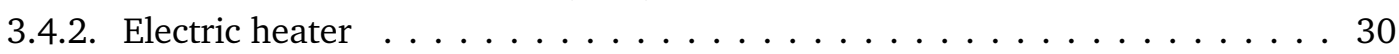

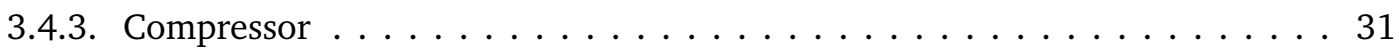

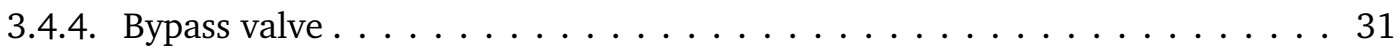

3.4.5. Supply air fan . . . . . . . . . . . . . . . . 31

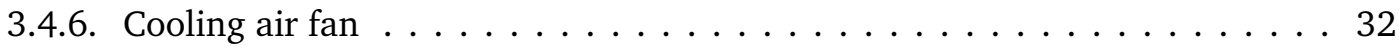

4. Grey-Box Modelling 33

4.1. Heat capacity and heat transfer coefficient of the tram model . . . . . . . . 33

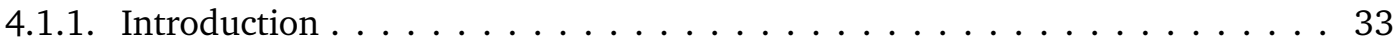

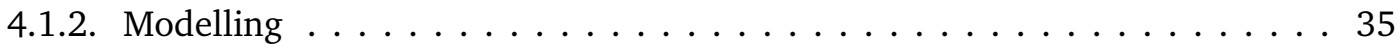

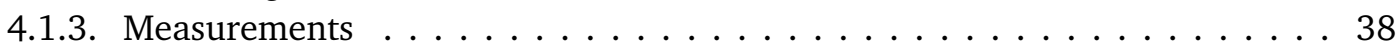

4.1.4. Assumptions . . . . . . . . . . . . . . . . . . . 40

4.1.5. Parameter estimation . . . . . . . . . . . . . . . 40

4.2. Further parameters of the tram model . . . . . . . . . . . . . 44

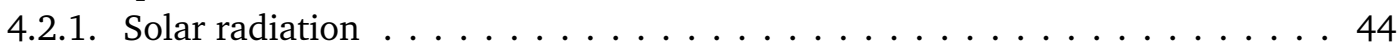

4.2.2. Passengers . . . . . . . . . . . . . . . . . . . 44

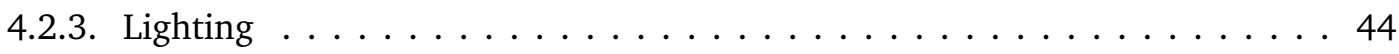

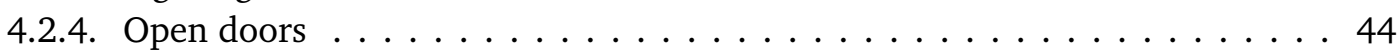

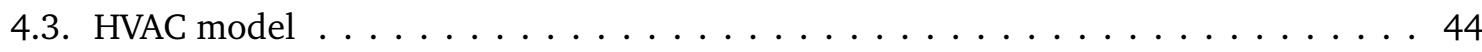

4.3.1. Electric heater . . . . . . . . . . . . . . . . . . . . 45

4.3.2. Compression refrigeration machine $(\mathrm{CRM}) \ldots \ldots \ldots \ldots \ldots$. . . . . . 45

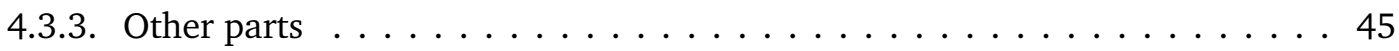

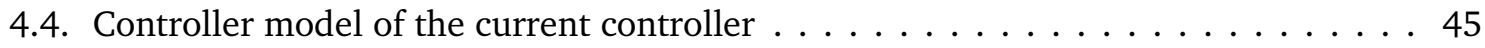

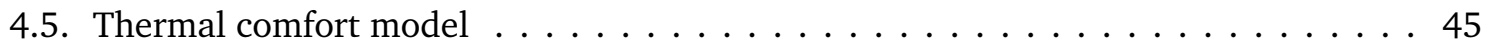

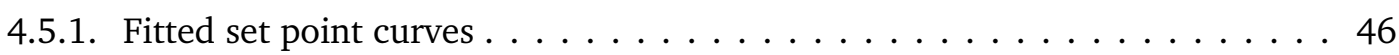

4.5.2. Predicted mean vote . . . . . . . . . . . . . . . . . . . . 49

5. Controller Design 53

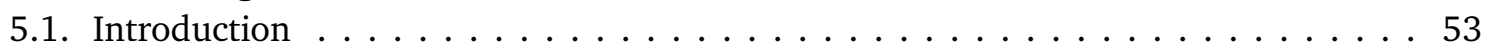

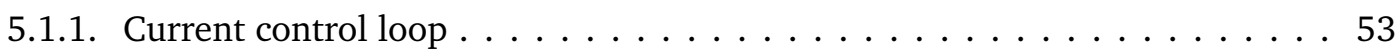

5.1.2. Improvement measures . . . . . . . . . . . . . . . . . . . 54

5.1.3. Control objectives . . . . . . . . . . . . . . . . . . . . 54

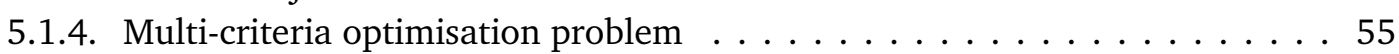

5.2. New control loop . . . . . . . . . . . . . . . . . . . . 56

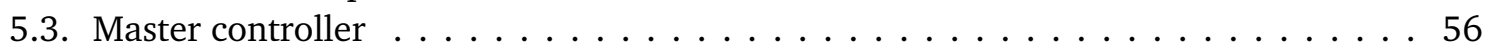

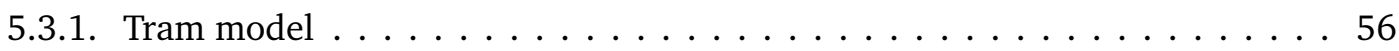

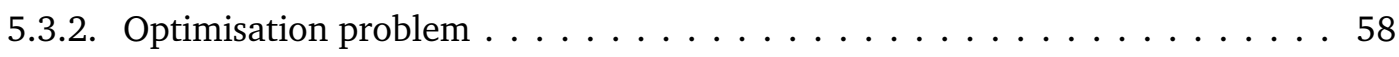

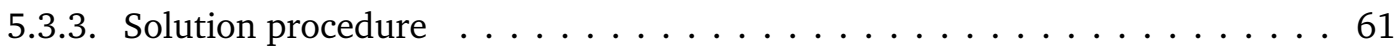

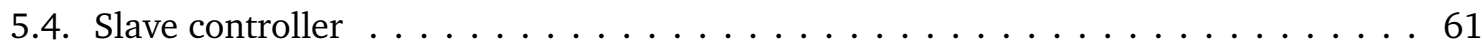

5.4.1. A linear HVAC model . . . . . . . . . . . . . . . . . . . . 62

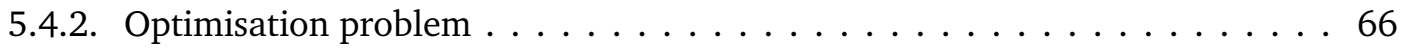

5.4.3. Simplified optimisation problem . . . . . . . . . . . 76 
5.4.4. HVAC model . . . . . . . . . . . . . . . . . . . . 80

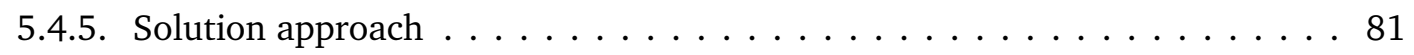

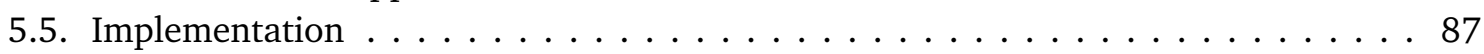

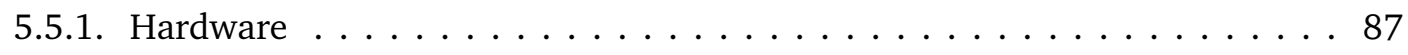

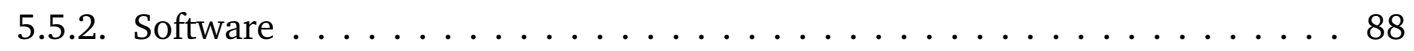

6. Results 91

6.1. Model Validation . . . . . . . . . . . . . . . . . . . . 91

6.1.1. Validation using Climatic Wind Tunnel Measurement Data . . . . . . . . . 91

6.1.2. Validation using On-Site Measurement Data . . . . . . . . . . . . . . . 92

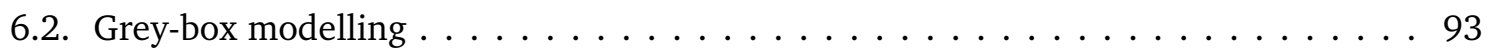

6.2.1. Model order . . . . . . . . . . . . . . . . . . . . . 93

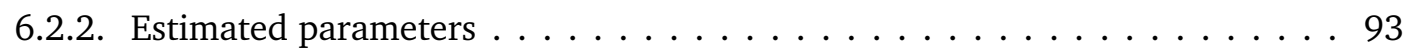

6.2.3. Generalized parameters . . . . . . . . . . . . . . . . . . . . . 94

6.2.4. Plausibility check . . . . . . . . . . . . . . . . . . . . . . 95

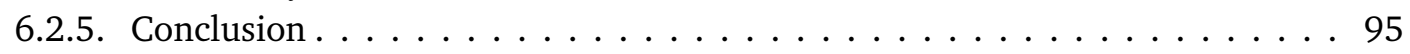

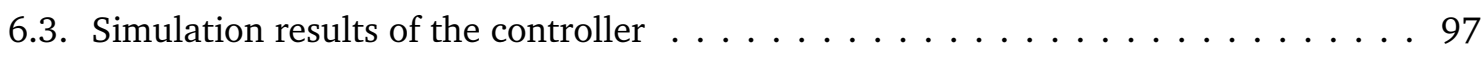

6.3.1. Master controller . . . . . . . . . . . . . . . . . . . . . 98

6.3.2. Slave controller . . . . . . . . . . . . . . . . . . . . . . . . . 99

6.4. Controller Validation . . . . . . . . . . . . . . . . . . . . . . . . . . 104

6.4.1. Individual tests . . . . . . . . . . . . . . . . . . . . . 104

6.4.2. Annual energy consumption . . . . . . . . . . . . . . . . . . 108

6.4.3. Conclusions of the controller validation . . . . . . . . . . . . . . . 108

$\begin{array}{lll}\text { 7. Outlook } & 113\end{array}$

A. Parameters $\quad 115$

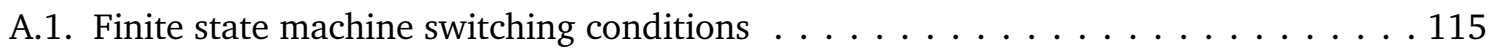

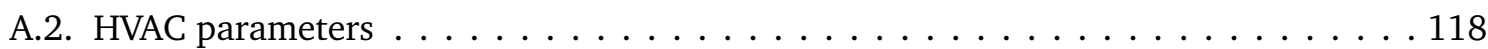

A.3. Parameters of the tram . . . . . . . . . . . . . . . . . . . . . . . 120

B. More results 121

B.1. Daily report . . . . . . . . . . . . . . . . . . . . . . . . . . . 121

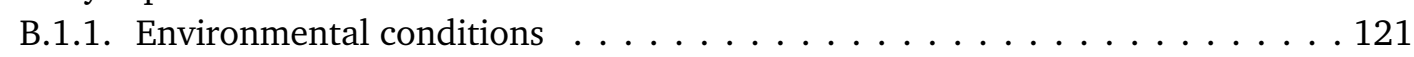

B.1.2. Tram . . . . . . . . . . . . . . . . . . . . . . . 124

B.1.3. HVAC . . . . . . . . . . . . . . . . . . . . . 126

B.2. Performance maps of the CRM . . . . . . . . . . . . . . . . . 129

C. Lists 137

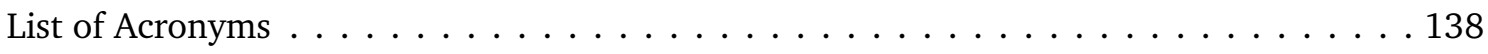

List of figures . . . . . . . . . . . . . . . . . . . . . . 140

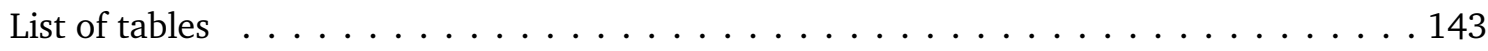

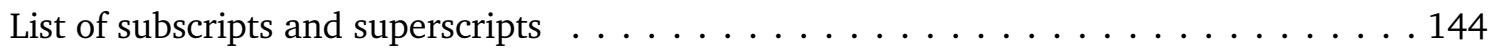

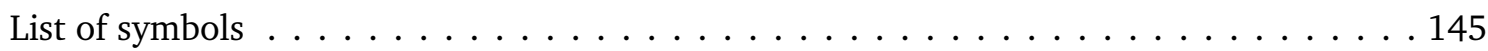

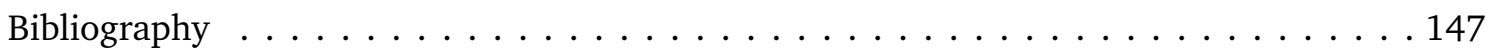


Curriculum vitae 


\section{1}

\section{Introduction}

In this chapter the motivation for this thesis is described first. Then the state of art in air conditioning of rail vehicles is discussed, from which the problem statement of this work is derived. At the end of the chapter, the most important points of the problem statement are summarised at a glance.

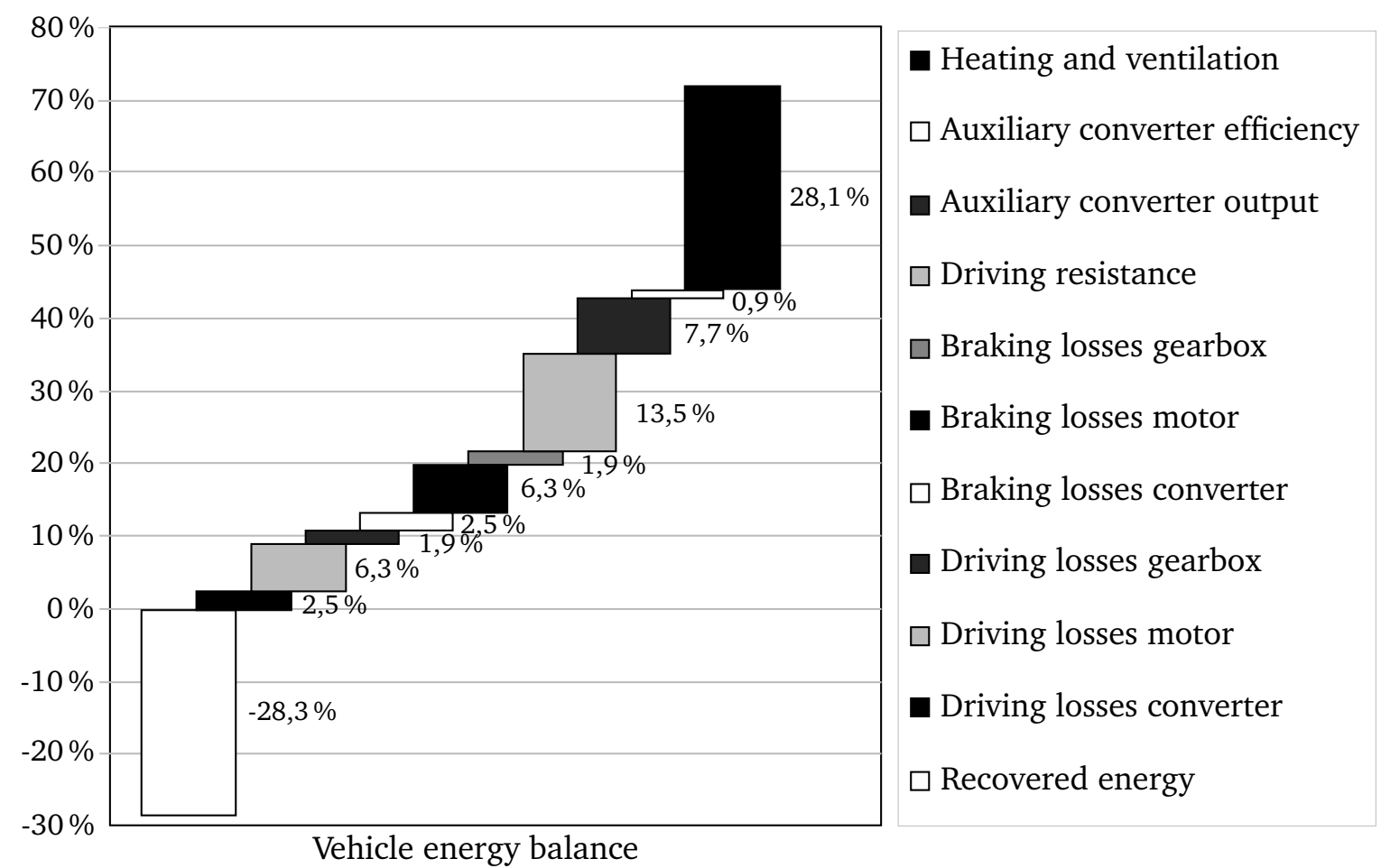

Figure 1.1. - Vehicle energy balance of a driving cycle of the Oslo metro [106, Figure 4.53]

\subsection{Motivation}

This motivation is also published in Hofstadter et al. [57]. 
Already in 1990, the Intergovernmental Panel on Climate Change announced that emissions resulting from humans would enhance the natural greenhouse effect, resulting on average in an extra warming of the Earth's surface [62]. The European Union thus follows the objective of increasing energy efficiency of products and services [34].

Energy efficiency of transport is important for the overall energy consumption. Many German researchers point out that transportation consumes $30 \%$ of the final energy consumption [81]. The modal split specifies (in percent) which means of transport travellers use. Percentages for public transport range from (almost) 0 to $60 \%$, depending on the region. Even though railway transport is already more energy efficient than other means of transport [98] and its efficiency was increased significantly in the past decade [70], a further increase is still possible. Researchers achieved many energy-saving measures for propulsion in the past years, by consistent lightweight design [46], driver assistance systems and hybridisation [47], for example.

Struckl [106] showed energy saving potential for rail vehicles using Oslo's subway as an example. HVACs consume about $30 \%$ of the total energy during driving cycles [106, see Figure 4.53]. Figure 1.1 shows the energy balance of a driving cycle of a metro, in which the proportion of heating and ventilation stands out during operation. So, they are the second largest energy consumer. Later research confirmed this extent. Isenschmid et al. [63] found that $33 \%$ of the total energy consumption is consumed by heating, ventilation and air conditioning systems (HVACs) in a Swiss train.

One approach to increase the energy efficiency of HVACs is to improve the ventilation efficiency. Air displacement ventilation can achieve this. Researchers have already used it successfully in buildings, aeroplanes [123] and recently in rail vehicles, too [13].

Another approach is to increase the energy efficiency of the heating, ventilation and air conditioning (HVAC) unit itself. Energy-saving measures known from other HVAC applications also apply to rail vehicles, such as demand-based ventilation, heating by a heat pump, variable frequency drive of the compressor and fine-grained control of the electric heater. These measures allow the HVAC unit to closely follow the demand, but with extra parts and fine-grained control, the complexity of the control problem rises. Model predictive control (MPC, also known as receding horizon control, rolling horizon planning and dynamic programming) is a proven method of control. The control action at each time step is obtained by solving an optimisation problem for the given time horizon. The resulting optimisation problem can often be formulated as a quadratic program [17] with real decision variables. Combinatorial optimization problems can sometimes be solved by (mixed) integer linear programming (MILP), which seeks the best result from a finite set of solutions is sought. Decision variables can be real, integer, binary or a combination thereof. Hybrid systems have both continuous and discrete dynamic behaviour. A vapour-compression refrigeration system can be described as a hybrid system, for example. In [74] modelling and optimal control of supermarket refrigeration systems are presented. Elliott at al. [31] use cascaded control for a multi-evaporator vapour compression cooling cycle, in which they set up the outer controller as a model predictive controller (MPC). Bejarano et. al. [9] use a suboptimal hierarchical control strategy to improve the energy efficiency of vapour-compression refrigeration systems. Also, [75] uses a binary variable (on-off) for temperature control in refrigerated transport systems. The authors of [16] introduce a distributed model predictive control scheme for supermarket refrigeration systems and [58] takes daily variations of i.a. electricity prices into account. Hybrid systems sometimes describe the building heating control problem, in which, for example, a mixed integer MPC is used for the integer variables as in [68] and [86]. Hybrid systems and their control also occur in energy 
management [67] and powertrains of (hybrid) vehicles [64].

The trend for improving thermal comfort and reducing the necessary energy consumption is set by the automotive industry. Muhammad et al. [87] studied the influence of thermostat level setting on fuel consumption and thermal comfort for the passenger compartment of a car. A new personal thermal comfort model is developed by Kim et al. [69] predicting an individual's thermal comfort response instead of the average response of a large population. Mathematical models and their validation for thermal comfort, air quality, and energy use are proposed by Kristanto et al. [73] for a car cabin. Since thermal comfort in the car influences the buying decision in the automotive industry, all concerns of this comfort are examined in detail. Bode et al. [14] researched the influence of the inlet angle of an air diffuser on the thermal comfort of passengers in a car. Velivelli et. al [114] examined the seat cooling distribution to further improve human thermal comfort in automotive environments.

The field of air conditioning for rail vehicles offers a considerable energy saving potential.

\subsection{State of art}

The manufacturing's work flow of a HVAC for rail vehicles looked like the following in 2010:

First, the later operator and the manufacturer of the vehicle together define thermal comfort for the vehicle in a specification sheet. The European Standards provide a general practice [60], from which one can deviate in an individual case. In which the minimum scope of functions for the HVAC (heating, cooling and ventilation) and a design point for the heating and air conditioning is defined. To the knowledge of the author, it is (currently) not common to specify the energy consumption of the HVAC in any way. With these requirements, the manufacturer of the HVAC writes a specification sheet, based on this paper, where the individual components are dimensioned. It is good engineering practice to design individual components rather a little bit too large than too small to meet the requirements in any case with a buffer. This is necessary, as the components are only available in distinct output sizes. The resulting partial load behaviour of the unit is usually not calculated. A controller for the HVAC is programmed, mostly based on empirical expert knowledge. The main focus is to meet the thermal comfort requirements in the vehicle. The controller does not consider the energy consumption of the HVAC, not even for internal purposes.

The overall system is usually tested based on a prototype in a climatic wind tunnel. And according to the specification, delivery will be accepted or rejected by the operator. At this point of time, changes to the configuration of the HVAC can still be made. Thereby only the thermal comfort is rated, even though energy consumption is usually measured in the climatic wind tunnel, too. Afterwards, further vehicles of the series are manufactured and delivered with the changed configuration. A further surveillance or logging of the vehicle will not take place during operation.

To the knowledge of the author, the operators do not have any or very little information which part consumes how much energy and which part of the public transport network consume how much energy and in which operating costs this energy consumption results. These figures are only partially available and they are not sufficient to allocate energy consumption to individual components and derive improvement measures for them. 


\subsection{Problem statement}

From the state of the art one can already formulate concrete questions, which need to be addressed in a wider project team. That is why not only the objectives of this work, but also the objectives of the projects are explained because they strongly interact with each other.

The objective of the project EcoTram is an optimisation of the thermal vehicle components in rail vehicles for urban transport while maintaining the thermal passenger comfort. Thus, improvement measures for the vehicle and its components are developed. Using climatic wind tunnel measurement, on-site measurements and simulations relationships between all relevant parameters are represented [29].

For that purpose, a simulation model is developed in this thesis. With that model, it should be possible to calculate the thermal comfort of passengers and the energy consumption of the HVAC dependent on environmental conditions (outdoor air (ODA), radiation, passenger load, etc.). Further, the effects of changes to the vehicle components and the operation conditions should be evaluated. For that purpose, model requirements have to be defined, the model needs to be split into submodels and these submodels have to be set up. Afterwards, it is necessary to fit the model to measurement data by estimating the parameters of the model.

The economically viable and technically feasible improvement measures are implemented on a non-commercial prototype in EcoTram II. Again, the energy consumption is measured in the climatic wind tunnel and on-site during passenger operation. New results are compared to existing measurement data and the achieved savings are shown [30].

From the suggested improvement measures follows that a new controller needs to be designed. It should use the previously created model to calculate smarter controlled variables for the HVAC. In doing so, it has to take both into consideration: thermal comfort and energy consumption. In addition various constraints of the HVAC and of the vehicle have to be taken into account, which are defined in the controller requirements. Based on the mathematical design, the controller needs to be ported to a Rapid Controller Prototyping Platform (RCPP). For this purpose, one has to define interfaces to the vehicle and to the HVAC.

\section{At a glance}

- Design a dynamic analytical model for the thermal vehicle components.

- Estimate parameters of this model.

- Validate the model with the estimated parameters.

- Develop a controller for the new HVAC.

- Show simulation results for the new controller.

- Implement the new controller onto a RCPP.

- Show measurement results from climatic wind tunnel measurements.

- Summarise the total energy saving. 


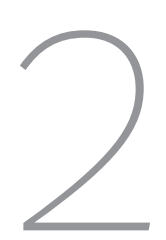

\section{Fundamentals}

This chapter briefly introduces the theoretical basis of this work. The modelling (see Chapter 3) needs knowledge of thermodynamics. Chapter 4 (Grey-box modelling) uses different optimisation methods and Chapter 5 needs knowledge of control engineering. For further information, the cited literature provides a good starting point.

\subsection{Thermodynamics}

The analytical model of the tram is formulated with energy and mass balance equations, which are based on the Laws of Thermodynamics. Heat exchange between various parts of the tram and between the tram and the environment represents the main influence. The HVAC or air conditioning system treats the humid air before it enters the tram.

\subsubsection{Laws of thermodynamics}

The energy balances stated in this thesis are based on the first law of thermodynamics. The second law allows calculating the cooling cycle of the compression refrigeration machine (CRM). So the following section cites both laws from [102, p. 63 and p. 122].

$1^{\text {st }}$ Law of thermodynamics The first law of thermodynamics represents the principle of energy conservation in its most generic form....

1. The energy $E$ of a thermodynamic system is an extensive state variable.

2. The energy balance

$$
d E=d_{e} W+d_{e} Q+d_{e}^{(m)} E
$$

defines heat $Q$. In which: $d E$ is the change of energy of the system, $d_{e} W$ the work performed, $d_{e} Q$ the supplied heat and $d_{e}^{(m)} E$ the energy input, which is linked to the supplied mass $d_{e} m$ ('material' energy input).

With respect to time, the energy balance is

$$
\frac{d E}{d t}=\dot{W}+\dot{Q}+\dot{E}^{(m)}
$$


in which $\dot{W}$ is work by time (power), $\dot{Q}$ is heat flow and $\dot{E}^{(m)}$ is material energy flow.

$2^{\text {nd }}$ Law of thermodynamics With the use of the term entropy, the second law follows from the following two partial statements:

1. For a closed system, which performs a reversible process, the entropy $S$ is defined by

$$
S_{2}-S_{1}=\int_{1 \rightarrow 2} \frac{d_{e} Q}{T}
$$

It is an extensive state variable (physical unit: $\mathrm{J} / \mathrm{K}$ ).

2. For a closed system, which performs an irreversible process, $S$ is

$$
S_{2}-S_{1}>\int_{1 \rightarrow 2} \frac{d_{e} Q}{T} .
$$

$T$ is the absolute temperature of energy storage, which introduces heat $d_{e} Q$ to the system, and integration is done over the process with initial state 1 and final state 2 .

\subsubsection{Heat exchange}

There are three types of heat exchange. These are

- heat conduction,

- convective heat transfer and

- heat radiation.

The authors of $[6,116]$ describe the different types.

\subsubsection{Humid air}

This subsection briefly summarises the topic of humid air from literature. The text follows the script of Glaninger [39]. [5, 41, 94, 102] provide extra information about humid air. According to Glaninger [39, p. 18], dry air is already a gas mixture consisting of about $21 \%$ of oxygen, $78 \%$ of nitrogen and $1 \%$ of argon. It does not change its composition in air conditioning systems and follows the laws of ideal gases. Humid air is a mixture of dry air and water vapour. Air conditioning technology (usually) considers a temperature range from $-30^{\circ} \mathrm{C}$ to $50^{\circ} \mathrm{C}$. The partial pressure of water vapour is small compared to the partial pressure of dry air. The mixture may also contain water droplets and ice particles. [39, p.18]

Dalton's law Dalton's law applies in a gas mixture. It states that the total pressure $p$ of a gas mixture consists of the partial pressures of the individual gases. Thus, it applies that: [39, p.18]

$$
p=p_{\text {air }}+p_{\text {vapour }},
$$

in which $p_{\text {air }}$ is the partial pressure of dry air and $p_{\text {vapour }}$ is the partial pressure of water vapour. [39, p.18] 
Relative humidity Relative humidity $\varphi$ is the ratio of the existing partial pressure of water vapour $p_{\text {vapour }}$ to the saturated vapour pressure of water $p_{S}$ at a given temperature $[39, \mathrm{p} .18 \mathrm{f}]$.

$$
\varphi=\frac{p_{\text {vapour }}}{p_{\mathrm{S}}(T)} \quad p_{\text {vapour }}=\frac{p \cdot Y}{0.622+Y}
$$

Absolute humidity The so-called moisture ratio $Y$, absolute humidity or humidity ratio [is] the ratio of the water vapour mass $m_{\text {vapour }}$ to the mass of dry air $m_{\text {air }}[39$, p.18f].

$$
Y=\frac{m_{\text {vapour }}}{m_{\text {air }}}
$$

Enthalpy of humid air For super-heated vapour follows [for the enthalpy $h$ of humid air] [39, p.19]:

$$
h_{1+Y}=c_{\text {air }} \cdot T+Y \cdot\left(c_{\text {vapour }} \cdot T+\Delta h_{\mathrm{V}}\right)
$$

In which $c$ is the heat capacity, in this case the specific heat capacity of dry air $c_{\text {air }}$ and of water vapour $c_{\text {vapour. }} \Delta h_{\mathrm{V}}$ is the heat of water vaporisation. [39, p.19]

Linearisation for the enthalpy of humid air One gets Equation (2.9) from resolving Equation (2.8)

$$
h_{1+Y}=c_{\text {air }} \cdot T+c_{\text {vapour }} \cdot T \cdot Y+Y \cdot \Delta h_{\mathrm{V}} .
$$

From the equation follows that the relation is nonlinear in $T$ and $Y$. Neglecting the term $c_{\text {vapour }} \cdot T \cdot Y$ for calculating the enthalpy of humid air results in an error of about 1 percent. The simplified relation for the enthalpy is now linear

$$
h_{1+Y}=c_{\text {air }} \cdot T+\Delta h_{\mathrm{V}} \cdot Y .
$$

This work uses the linear relation from Equation (2.10) unless clearly stated otherwise.

Energy input for heating and cooling ... the energy needed for heating or cooling [can be] easily calculated by multiplying the mass flow of dry air with the enthalpy difference between entry and exit. [39, p. 19]

$$
\dot{Q}=\dot{m}_{\text {air }} \cdot\left(h_{1}-h_{2}\right),
$$

Thus, $\dot{Q}$ describes the heat flow in $\mathrm{J} / \mathrm{s}$.

Rate of water vapour The rate of water vapour can be calculated by [39, p. 19f].

$$
\dot{E}^{\mathrm{W}}=\dot{m}_{\mathrm{air}} \cdot\left(Y_{1}-Y_{2}\right) .
$$

Thus, $\dot{E}^{\mathrm{W}}$ describes a mass flow in $\mathrm{kg} / \mathrm{s} .[39, \mathrm{p} .19 \mathrm{f}]$ 


\subsubsection{Heating, ventilation and air conditioning system}

Glaninger [39, p.8] defines an HVAC, in short an air conditioning system or more generally an air treatment plant as:

These are plants influencing the dust content, the temperature and the humidity of the indoor air as well as the outside air supply and the air movement in the room in such a way that a required indoor climate is achieved and kept [39, p. 8].

This work often uses the term thermal comfort instead of the required expression of indoor climate. The use of air conditioning covers all fields of engineering which need a high air quality. It covers classic application in buildings (residential, office and industrial buildings) and transport (automobiles, buses and trains), but also include special applications like clean room technologies. Experts classify air conditioning systems into different groups by design and capacities associated with that design. One distinguishes between full and partial designs. A fully designed air conditioning has the capacity to [39, p. 10]:

- heat,

- cool,

- ventilate,

- dehumidify and

- humidify.

Moreover, air cleaning is another capacity and other special capacities are possible, too. While the size and the weight of an HVAC plays a minor role in the building technology, it is important in trams. Thus, not all energy saving measures for buildings apply to trains as well.

For the building technology, there is comprehensive literature to calculate the energy consumption during the year. To the knowledge of the author this literature does not exist for trains. [94] is the reference book in air conditioning. European Norm (EN) 12831 [111] and VDI 2078 [8] show (design) calculations of heating and cooling loads. The course material of Glaninger's class [39] also covers these topics.

\subsubsection{Thermal comfort}

Air conditioning systems achieve environmental conditions that occupants consider as comfortable. Building engineers use an approach to calculate how occupants sense temperature [61]. They calculate the value of the so-called predicted mean vote (PMV) from air temperature, mean radiation temperature, relative humidity, airspeed, metabolic rate and clothing insulation of occupants. The PMV ranges from -3 (very cold) over 0 (neutral) to +3 (very hot), in which a range between -0.5 and 0.5 is comfortable. Percentage persons dissatisfied (PPD) is a value that follows from PMV. It shows the percentage of dissatisfied persons, dissatisfied with the thermal comfort. Formulas for calculating both values are nonlinear.

The state of the art for climate control in trams uses simpler approaches. The standard for urban and suburban trains [60] provides a set point curve for the indoor air temperature as a function of the outdoor air temperature. In addition, constraints apply to supply air temperatures, airspeeds, surface temperatures and the temperature distribution in the tram apply. Something similar applies to mainline trains [59]. 
Usually, one of both standards [59] or [60] is the contractual basis between train producers and train operators. However, it is common that contracts deviate in some points from the relevant standard. The Viennese tram operator has the possibility to adapt the set point curve, for example.

\subsection{Optimisation}

There are often different interpretations of the term optimisation so that this subsection defines it. Most of the optimisation problems are unsolvable, but there exists a sound basis for convex optimisation. One usually assumes the optimisation variable to be $x \in \mathbb{R}$. If this assumption does not hold, one speaks of (mixed) integer optimisation.

Boyd and Vandenberghe [17] define an optimisation problem as follows: A mathematical optimisation problem, or just optimisation problem, has the form

$$
\begin{array}{ll}
\text { minimize } & f_{0}(x) \\
\text { subject to } & f_{i}(x) \leq b_{i}, i=1, \ldots, m
\end{array}
$$

Here the vector $x=\left(x_{1}, \ldots, x_{n}\right)$ is the optimisation variable of the problem, the function $f_{0}: \mathbb{R}^{n} \rightarrow$ $\mathbb{R}$ is the objective function, the functions $f_{i}: \mathbb{R}^{n} \rightarrow \mathbb{R}, i=1, \ldots, m$ are the (inequality) constraint functions, and the constants $b_{1}, \ldots, b_{m}$ are the limits, or bounds, for the constraints. We call a vector $x^{\star}$ optimal, or a solution of the problem defined in Equation (2.13), if it has the smallest objective value among all vectors that satisfy the constraints: for any $z$ with $f_{1}(z) \leq b_{1}, \ldots, f_{m}(z) \leq b_{m}$, we have $f_{o}(z) \geq f_{0}\left(x^{\star}\right)[17$, p.1].

\subsubsection{Convex optimisation}

Using convex optimization is, at least conceptually, very much like using least-squares or linear programming. If we can formulate a problem as a convex optimization problem, then we can solve it efficiently, just as we can solve a least-squares problem efficiently. With only a bit of exaggeration, we can say that, if you formulate a practical problem as a convex optimization problem, then you have solved the original problem. [17, see Section 1.3.2]

Boyd and Vandenberghe [17] define convex optimisation in their fundamental book as follows: A convex optimisation problem is one of the forms

$$
\begin{array}{ll}
\text { minimize } & f_{0}(x) \\
\text { subject to } & f_{i}(x) \leq b_{i}, \quad i=1, \ldots, m,
\end{array}
$$

where the functions $f_{0}, \ldots, f_{m}: \mathbb{R}^{n} \rightarrow \mathbb{R}$ are convex, i.e. satisfy

$$
f_{i}(\alpha x+\beta y) \leq \alpha f_{i}(x)+\beta f_{i}(y)
$$

for all $x, y \in \mathbb{R}^{n}$ and all $\alpha, \beta \in \mathbb{R}$ with $\alpha+\beta=1, \alpha \geq 0, \beta \geq 0$. The least-squares problem and the linear programming problem are both special cases of the general convex optimisation problem. $[17, \mathrm{p} .7]$ 


\subsubsection{Mixed integer optimisation}

The section above assumes that $x \in \mathbb{R}$ holds for the optimisation variable $x$. This section shows what applies if this assumption is false.

For the given vectors $x^{T}=\left(x_{1}, \ldots, x_{n_{c}}\right)$ and $y^{T}=\left(y_{1}, \ldots, y_{n_{d}}\right)$, which represent $n_{c}$ continuous and $n_{d}$ integer variables, the contiguous subset $X \subseteq \mathbb{R}^{n_{c}}$, discrete and bounded subset $U \subseteq \mathbb{Z}^{n_{d}}$, the composite vector $\mathbf{x}_{\oplus}^{T}=\mathbf{x}^{T} \oplus \mathbf{y}^{T}$, an objective function $f(x, y), n_{e}$ equality constraints and $n_{i}$ inequality constraints $g(x, y)$, the optimisation problem

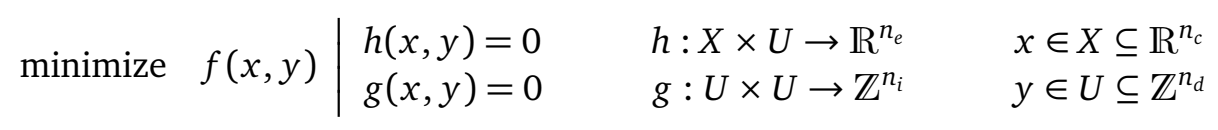

is a mixed integer nonlinear optimisation problem (MINLP) if at least one of the functions $f(x, y)$, $g(x, y)$ or $h(x, y)$ are nonlinear and $n_{c}>0$ and $n_{d}>0$ apply. With $n_{c}=0$ and $n_{d}>0$ the problem is an integer nonlinear optimisation problem. [65, p.14]

\subsubsection{Multi-criteria optimisation}

Kallrath [65] wrote about multi-criteria optimisation:

Optimisation treats a problem statement with a clearly defined objective function. In practice, however, one often meets questions where a model considers conflicting objectives....

An approach to solve such multi-criteria optimisation problems ... is to map all objectives to a common measurable variable. ... A useful approach for multi-criteria optimisation is to consider pareto-optimal solutions, which these are as follows: In a multi-criteria optimisation problem with $n$ to maximising objective criteria $Z-i(x), i=1, \ldots, n$ is a feasible solution $x^{\star}$ pareto-optimal solution if there is no other feasible solution $x$ with $Z_{i}(x) \geq Z_{i}\left(x^{\star}\right)$ for all $i$ and $\max _{i} \operatorname{sign}\left\{Z_{i}(x)-Z_{i}\left(x^{\star}\right)\right\}=1$, i.e. there is no other feasible solution which, in all objectives criteria, it at least as good as the solution $x^{\star}$ and is not better in at least one objective criterion [65, p.160].

Various fields are looking for pareto-optimal solutions. One field of application is the modern portfolio theory according to Markowitz [105, p.177] and the Risk-Return-Diagram.

A so-called Risk-Return-Diagram contains all individual investments ... from which portfolios can be formed. ...Risk is shown on the x-axis and return on the y-axis [105, p.177]. Figure 2.1 shows this context.

A special solution approach for multi-criteria optimisation problems is that all objectives must meet certain targets ... as good as possible. ... The formed objective function seeks to minimise all deviations of the targets from their goals. The solutions resulting from the goal programming approach are pareto-optimal. [65, p.160]

One distinguishes two approaches:

1. the Archimedean approach and

2. the pre-emptive ... approach [65, p.160].

The Archimedean approach penalises deviations from the target with weights or penalty terms, thus using a weighted sum of the objective functions.... The pre-emptive approach sorts goals by significance and priority, in which the goal $i$ is infinitely more important than goal $i+1$. [65, p.161] 


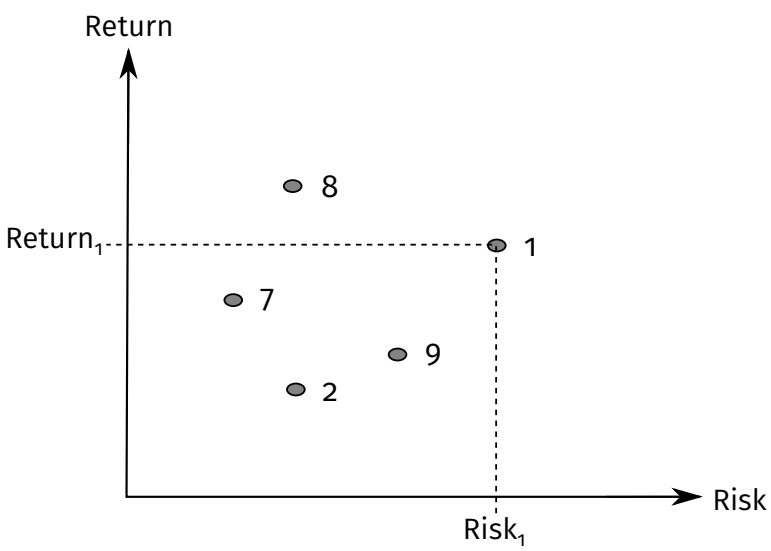

Figure 2.1. - Risk-Return-Diagram. 7 and 8 are efficient portfolios. 1, 2 and 9 are not efficient [105, Fig. 7.1].

\subsection{Control engineering}

This thesis assumes basic knowledge of control engineering as taught in [15, 109, 110]. This chapter summarises some of the basics used: General State Space System, Cascaded Control and model predictive controller (MPC).

\subsubsection{General state space system}

Thus, the general form of the state space representation of a linear, time-invariant dynamic system of order $n$ is

$$
\begin{aligned}
& \dot{x}(t)=\mathbf{A} x(t)+\mathbf{B} u(t), \quad x\left(t_{0}\right)=x_{0} \\
& y(t)=\mathbf{C} x(t)+\mathbf{D} u(t) .
\end{aligned}
$$

In which

$$
\begin{gathered}
x(t)=\left[\begin{array}{c}
x_{1}(t) \\
\vdots \\
x_{n}(t)
\end{array}\right] \in \mathbb{R}^{n} \quad \text { state vector } \\
u(t)=\left[\begin{array}{c}
u_{1}(t) \\
\vdots \\
u_{r}(t)
\end{array}\right] \quad \in \mathbb{R}^{m} \quad \text { input vector } \\
y(t)=\left[\begin{array}{c}
y_{1}(t) \\
\vdots \\
y_{m}(t)
\end{array}\right] \quad \in \mathbb{R}^{o} \quad \text { output vector }
\end{gathered}
$$




$$
\begin{gathered}
\mathbf{A}=\left[\begin{array}{ccc}
a_{11} & \ldots & a_{1 n} \\
\vdots & & \vdots \\
a_{n 1} & \ldots & a_{n n}
\end{array}\right] \in \mathbb{R}^{n \times n} \text { state matrix } \\
\mathbf{B}=\left[\begin{array}{ccc}
b_{11} & \ldots & b_{1 m} \\
\vdots & & \vdots \\
b_{m 1} & \ldots & b_{m m}
\end{array}\right] \in \mathbb{R}^{n \times m} \text { input-to-state matrix } \\
\mathbf{C}=\left[\begin{array}{ccc}
c_{11} & \ldots & c_{1 n} \\
\vdots & & \vdots \\
c_{o 1} & \ldots & c_{o n}
\end{array}\right] \in \mathbb{R}^{o \times n} \text { state-to-output matrix } \\
\mathbf{D}=\left[\begin{array}{ccc}
d_{11} & \ldots & d_{1 m} \\
\vdots & & \vdots \\
d_{o 1} & \ldots & d_{o m}
\end{array}\right] \in \mathbb{R}^{o \times m} \text { feed-through matrix }
\end{gathered}
$$

Equation (2.17) is the (vectorial) state differential equation or short state equation. It describes the dynamic of the system....

The state matrix $\mathbf{A}$ also contains the complete information about the Eigen behaviour and therefore about on the stability of the system, for example. So the input matrix B describes how external excitations (the inputs) affect the system.

Equation (2.18) is described as output or observer equation. It connects states to the outputs by the matrix $\mathbf{C}$, a (purely static) linear combination of the states. Sometimes there is a directly proportional effect of inputs on outputs by the feed-through matrix B. Those are bi-proper systems $[110$, p.3ff].

\subsubsection{Cascaded control}

In many control systems one or more variables, except for the controlled variable, can be measured as auxiliary control, which can support the control by feed-forward signals or by auxiliary controllers. The control loop is a cascaded controller because of the sequence of controllers. [15, p.157]

The slave control loop should compensate the influence of disturbance $z_{2}$ as fast as possible so that it has hardly influence on the master controller. ... Process engineers and electric drive engineers mainly use cascaded control. According to Wikipedia [121], an example application of cascaded control is the room temperature control, in which the supply air temperature is used as an auxiliary control variable.

\subsubsection{Model predictive control}

Conventional control engineering methods, such as PID control, responds to deviations of the actual value from the setpoint. The advanced model predictive control uses available knowledge about the control task to find the best solution. Mathematicians express the MPC problem as a quadratic problem, a subfield of convex optimisation.

This section explains the difference between conventional and advanced control methods for an everyday control task and it summarises the most important theory. Then the section provides a 
brief look into MPC's development history and introduces the two most important MPC classes.

\section{A motivating example}

To understand the difference between the control methods, it is helpful to understand how humans solve an everyday control task. Many people go to work by car. It is such a daily exercise the the outstanding control performance behind this task does not need much attention. You develop a feeling for the car through frequent practice. You know how the car reacts in common situations and you know the car's limits. On often travelled routes one knows the dangerous spots, spots where strong crosswind occur or where snow and ice collect, most likely in winter. Obviously, one will reduce the car's speed early before an effect is noticeable. MPC tries to reproduce this control. For that purpose, the controller needs a model of the plant (like a feeling for the car) and can consider occurring constraints. Extra knowledge about disturbances (crosswinds) can be considered as well. In contrast, a PID controller only reacts to current deviations of the set point. Kozek [72] gave this example during his speech to clearly explain the MPC idea.

\section{MPC algorithm formulation}

Garcia et al. [38] explain the MPC algorithm as follows: The name "Model Predictive Control" arises from the manner in which the control law is computed. At the present time $t$ the behavior of the process over a horizon $\tau$ is considered, Using a model the process response to changes in the manipulated variable is predicted. The moves of the manipulated variables are selected such that the predicted response has certain desirable characteristics. Only the first computed change in the manipulated variable is implemented. At time $t+1$ the computation is repeated with the horizon moved by one time interval. [38, The names of the variables have been adjusted.]

\section{History of development}

Grüne et al. [42] show a detailed explanation of MPC's development.

\section{Rough classification}

Experts classify MPC into several categories. The most important classification is by problem type: linear or nonlinear. Linear MPC is a thoroughly proven and tested method. In the past years, important progress was also made in nonlinear MPC so far but only a few special problems are solvable. The computational effort is usually higher than for linear MPC. Multiple tools exist for solving the MPC problem on modern architectures (x86) in several minutes. Challenges arise where these modern architectures are not available because embedded systems of much lower performance need to be used. Another challenge comes from the short calculation time, which must not exceed $50 \mathrm{~ms}$ even in the worst case. 



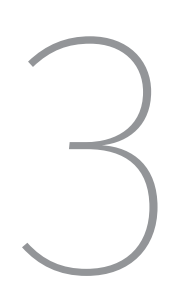

\section{Modelling}

This chapter describes the modelling of tram parts that are relevant for the thermal comfort. It is based on prior publications of the author, which are listed in the first part of this chapter. Then an introduction explains the objectives, the closed control loop, the resulting structure of the model and the method. The following sections explain the individual submodels: the model of the vehicle, the air conditioning and the controller. Chapter 4 explains the method for estimating missing parameters of the model.

References Section 1.2 lists general modelling references. The scientific fundamentals (e.g. thermodynamics) can be found in Section 1.3.

Prior publications This chapter describes information already published by the author. It contains the following scientific contribution

- Hofstädter et al. [54],

- Hofstädter and Kozek [51],

- Hofstädter and Kozek [52] and

- Hofstadter et al. [57].

This chapter contains extra information created for internal purposes or as project documentation of similar or overlapping content. It also includes the following documents:

- Deliverable for work package (WP) 1.5.1 [48]

- Deliverable for WP 1.5.2 and 1.5.3 [49]

- Thermal Vehicle Model Report [53].

This chapter summarises all published and unpublished contributions on the thermal modelling of rail vehicles. It extends the models in some matters, supplements the model descriptions and corrects some previous errors. It also introduces a consistent nomenclature for the following chapters of this work.

\subsection{Introduction}

Before starting with the modelling, this section defines the basis. It describes the scope of modelling and the modelled train. Then, it discusses the closed control loop, which leads to the (main) parts of the model. The last section of this section describes the method used. 


\subsubsection{Scope of modelling}

The scope of the simulation model follows from the objective of this work (see Chapter 1), which leads to the model's requirements.

\section{Modelling objective}

The modelling objective is calculating the energy consumption of the HVAC and the thermal comfort of the passengers, depending on the environmental conditions of the tram.

\section{Modelling requirements}

Work package 1.5.1 of the EcoTram project defines the requirements of the model [48]. The following paragraph summarises them briefly. Besides the modelling objective, the main objectives are a satisfying accuracy and robustness of the model. This means for example, that the simulation can reproduce the temperature measurements and that the temperature deviation is smaller than $2{ }^{\circ} \mathrm{C}$. A robust simulation model produces true results even if certain measurement errors of the simulation inputs (environmental conditions) occur. Optional objectives might later play an important role. The modelling describes a tram, but it should be possible to generalise the results for other trains, that are trains are metros, light rail, regional or mainline vehicles, for example. The list does not include locomotives as some modelling requirements are not met. The requirements also list some non-objectives like no platform independent simulation software and no simulation of fast transients. Pressure drops inside the tram, for example, which result form opening and closing doors, are considered as fast transients.

\subsubsection{Closed control loop}

Train, HVAC and controller form a closed control loop of climiatisation. In the train a specific state (that is a temperature) of indoor air $y$ exists, also called actual value. Various sensors (that are thermocouples) make measurements and send them to the controller. The controller produces a control error $\boldsymbol{e}$ from comparing set points $w$ and measurements. Then the controller calculates a control signal $u$ and sends it to the HVAC. The HVAC properly conditions the supply airflow to the train to minimize the control error. This conditioning of the air needs electrical energy. Various disturbances $z$ act on the train and on the HVAC so that the indoor air reaches a new state.

Figure 3.1 shows a schematic representation of the closed control loop of the climate control of a train. Section 4.5.2 discusses the closed control loop in more detail.

\subsubsection{Structure of the model}

Figure 3.1 shows the three major parts of the entire model. It consists of models for

- one section of the tram,

- the HVAC and

- the controller.

All parts of the model use a similar modelling approach. The following subsection describes it. 


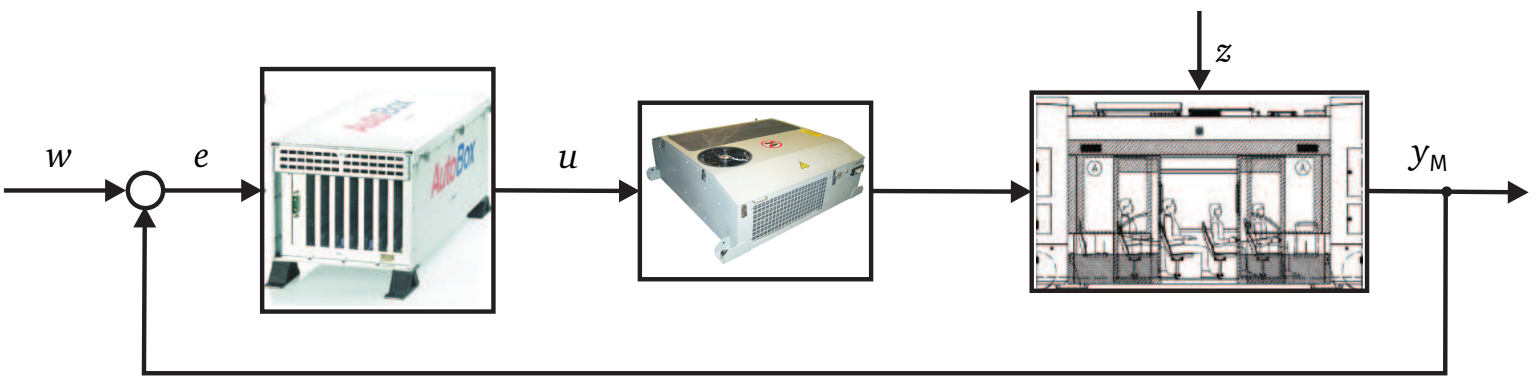

Figure 3.1. - Closed control loop of the climatisation of the vehicle

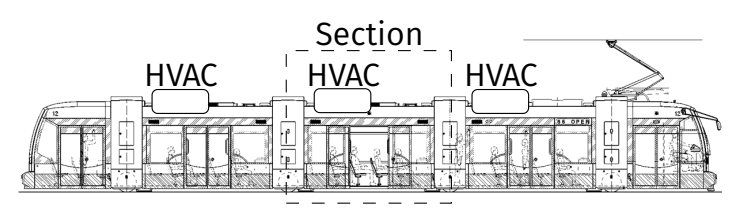

(a) The ULF 151 A1 with three HVACs and one highlighted section.

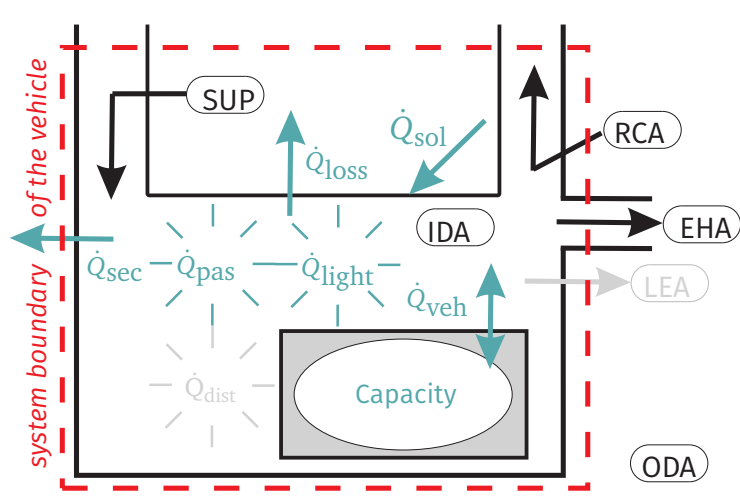

(b) Heat and mass flows are plotted for one section of the tram

Figure 3.2. - Schematic sketches of the tram

\subsubsection{Method}

The underlying physical or logical relations were established for every part of the model (e.g. heat transfer in the vehicle and switching conditions of the controller).

When preparing the basic equations, an approach of low complexity was chosen that quantitatively reflects the correlations. The measurements should allow estimating the needed parameters. The model must not need more parameters than measurements can provide. The mathematical relations were implemented in the simulation environment Matlab ${ }^{\circledR}$.

The following three sections describe the analytical model of a tram section, the HVAC and the controller.

\subsection{Tram model}

The studied rail vehicle is the Ultra Low Floor tram A1 made by Siemens AG Österreich and managed by the Wiener Linien GmbH \& Co KG in Vienna. The tram is about $24 \mathrm{~m}$ long, $2.4 \mathrm{~m}$ wide and $3 \mathrm{~m}$ high with an unloaded weight of $30 \mathrm{t}$. It is split into three similar sections with an added bow and tail section. Figure 3.2a shows a schematic of the tram. There are twelve seats in every section, five more seats in the tail section and a wheelchair space in the bow, resulting in a 
total of 42 seats. There is also space for 94 standing passengers. All three main sections of the tram contain their own HVAC unit. Each HVAC can heat, ventilate, cool and dehumidify the tram. Vossloh-Kiepe GmbH designed the HVACs.

So the studied tram consists of three sections. Since each of the sections has an identical structure, the modelling of only one section is explained below. Simulating the entire tram works by calculating three sections. The modular design has the advantage that it can easily be generalised to other trains. The modelling only considers the passenger area.

The driver's cab is spatially separated from it and has its own HVAC. In each of these sections, the following variables are to be calculated

- indoor air temperature $T_{\text {IDA, }}$,

- temperature of the carbody $T_{\text {veh}}$,

- absolute humidity of indoor air $Y_{\mathrm{IDA}}$ and

- carbon dioxide concentration of indoor air $\sigma_{\text {IDA }}$.

They all affect the thermal passenger comfort and/or the energy consumption of the HVAC. The following sections explain both the balance equations used for each of these quantities and the heat and mass flows occurring in these balances.

\subsubsection{Balance equations}

In the tram, or in each section of the tram, the four variables mentioned above are calculated. Figure $3.2 \mathrm{~b}$ shows a schematic sketch of the heat and mass flows of a tram section as well as the balance boundaries of the model.

\section{Indoor air temperature}

Calculating the indoor air temperature $\dot{T}_{\text {IDA }}$ uses the first law of thermodynamics [5, chap. 2] and applies it as

$$
C_{\mathrm{IDA}} \cdot \dot{T}_{\mathrm{IDA}}=\sum \dot{Q}_{i}+\sum \dot{E}_{i}
$$

$C_{\text {IDA }}$ is the heat capacity of the interior. $\dot{Q}$ is a heat flow and $\dot{E}$ is an enthalpy flow. Any work performed in the interior is neglected $(\dot{W}=0)$. The following subsections explain calculating itemised heat $\dot{Q}_{i}$ and mass flows $\dot{E}_{i}$.

\section{Temperature of the carbody}

Equation (3.2) describes the temperature of the carbody

$$
C_{\text {veh }} \cdot \dot{T}_{\text {veh }}=\dot{Q}_{\text {veh }} \text {. }
$$

$C_{\text {veh }}$ is the heat capacity of the carbody and $\dot{T}_{\text {veh }}$ is the temperature of this heat capacity. Hence, the modelling assumes that heat is only transferred between the heat capacity of the tram and indoor air (see Figure 3.2b). 


\section{Absolute humidity}

The absolute humidity of the indoor air $Y$ can be calculated from the partial mass balance of water. From this Equation (3.3) follows,

$$
V_{\mathrm{IDA}} \cdot \rho_{\mathrm{IDA}} \cdot \dot{Y}_{\mathrm{IDA}}=\sum \dot{E}_{\text {in }, i}^{\mathrm{W}}-\sum \dot{E}_{\text {out }, i}^{\mathrm{W}}
$$

where $V$ is the volume of the interior and $\rho$ the density of the indoor air. $\dot{E}_{i n, i}$ describes the various incoming material flows of water and $\dot{E}_{\text {out }, i}$ the outflowing material flows.

\section{Carbon dioxide concentration of the interior}

The indoor carbon dioxide concentration can be calculated from the partial mass balance of carbon dioxide. From this Equation (3.4) follows

$$
V_{\mathrm{IDA}} \cdot \rho_{\mathrm{IDA}} \cdot \dot{\sigma}_{\mathrm{IDA}}=\sum \dot{E}_{\mathrm{in}, i}^{\mathrm{CO} 2}-\sum \dot{E}_{\mathrm{out}, i}^{\mathrm{CO} 2}
$$

Again, $\dot{E}_{\text {in }, i}$ describes the incoming material flows and $\dot{E}_{\text {out }, i}$ the outflowing material flows. In this case, superscript ${ }^{\mathrm{CO} 2}$ shows the material flows of $\mathrm{CO} 2$. This is an identical approach to the approach of the absolute humidity calculation.

\section{Mass balance of the air}

The interior room satisfies the mass balance of air.

$$
\dot{m}_{\mathrm{SUP}}=\dot{m}_{\mathrm{IDA}}+\dot{m}_{\mathrm{ODA}}
$$

Where $\dot{m}$ represents a mass flow. Table 3.1 shows the abbreviations of the types of air. Equation (3.5) describes a stationary balance; transient conditions (e.g. pressure drops through open doors) are not considered.

\subsubsection{Heat flows}

Figure $3.2 \mathrm{~b}$ shows a schematic diagram of the tram and depicts the occurring heat and mass flows in a section. The following is a list of the used descriptions:

- Outer shell of the tram $\dot{Q}_{\text {loss }}$

- Interior room and carbody $\dot{Q}_{\mathrm{veh}}$

- Section $\dot{Q}_{\text {sec }}$

- Solar radiation $\dot{Q}_{\text {sol }}$

- Passengers $\dot{Q}_{\text {pas }}$

- Electric lighting $\dot{Q}_{\text {light }}$

Table 3.1 lists the abbreviations of the mass flows of air; it uses the English abbreviations from EN 13779 [88]. 


\begin{tabular}{lll}
\hline & Table 3.1. - Description of air flows in the tram (according to [88]) \\
\cline { 2 - 3 } Abbreviation (Engl.) & German & \multicolumn{2}{c}{ Description } \\
\hline SUP & Zuluft & supply air \\
IDA & Innenraumluft & indoor air \\
ODA & Außenluft & outdoor air \\
RCA & Umluft & recirculation air \\
EHA & Fortluft & exhausted air \\
LEA & Leckageluft & leakage air \\
\hline
\end{tabular}

\section{Outer shell of the tram}

For the heat flow through a flat wall under stationary conditions [5, Eq. 2.12] (like those assumed here) it is

$$
\dot{Q}_{\text {loss }}=k \cdot O_{\text {loss }} \cdot\left(T_{\text {IDA }}-T_{\text {ODA }}\right) \text {, }
$$

where $O_{\text {loss }}$ is the area of the outer shell of the tram and $T$ is the air temperature. For the heat transfer coefficient follows in general [116, Eq. 2.12] that

$$
\frac{1}{k}=\frac{1}{\alpha_{\text {in }}}+\frac{\lambda}{l}+\frac{1}{\alpha_{\text {out }}}
$$

$\lambda$ is the heat conductivity of the wall, $l$ the thickness of the wall and $k$ the heat transfer coefficient between the surface of the wall and air. The indices in stands for the inside and out for the outside. The values of $\lambda$ and $k$ are both material characteristics. While $\lambda$ is constant in this simplified context, this does not apply to $k$. $k$ depends on the flow conditions. The tram speed $v$ describe the flow conditions for $\alpha_{\text {out }}$. The resulting relation is usually non-linear.

\section{Interior room and carbody}

To better describe the non-linear temperature behaviour of the tram, the model includes an extra heat capacity of the tram. It interacts only with the interior room. The heat transfer between this capacity and the interior room is

$$
\dot{Q}_{\mathrm{veh}}=k_{\mathrm{veh}} \cdot\left(T_{\mathrm{IDA}}-T_{\mathrm{veh}}\right) .
$$

$k_{\mathrm{veh}}$ is the heat transfer coefficient between the interior and the surface of the modelled heat capacity of the vehicle body $C_{\text {veh. }}$. However, a split-up into two factors, as done in Equation (3.6), is not reasonable since neither the surface nor the heat transfer coefficient can be chosen distinctively. [56] 


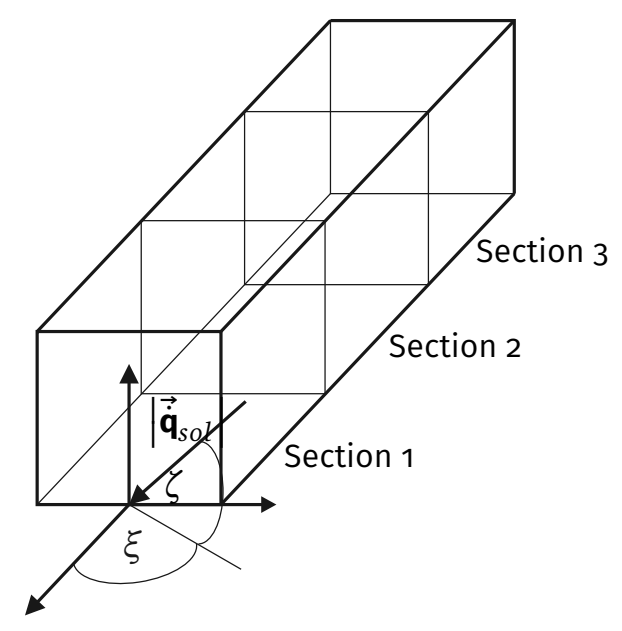

Figure 3.3. - Drawing of solar radiation into the vehicle

Section

For the heat flow between the sections of the tram, a weak static coupling, depending on the temperature of the connected sections, was assumed.

$$
\dot{Q}_{\mathrm{sec}}=\operatorname{sign}\left(T_{\mathrm{IDA}, 1}-T_{\mathrm{IDA}, 2}\right) \cdot k_{\mathrm{sec}}
$$

At a positive leading sign, the heat transfer takes place from section 1 to section 2, between section 2 and 3 the relation is analogous. If the heat exchange is neglected, instabilities occur in the model. There exists perfect isolation between the separate sections. If the measured airspeed between the modules is used to calculate the heat flow, the unsigned measurement yields a too big coupling because the leading sign can only be assumed. One will also obtain a too large coupling if $\left(T_{\mathrm{IDA}, 1}-T_{\mathrm{IDA}, 2}\right)$ is used instead of $\operatorname{sign}\left(T_{\mathrm{IDA}, 1}-T_{\mathrm{IDA}, 2}\right)$.

\section{Solar radiation}

In the model the sun is a parallel black emitter, which radiates a specific heat flow. Thus, solar radiation is a vector in spherical coordinates, in which the length $\left|\overrightarrow{\mathbf{q}}_{\text {sol }}\right|$ and both angles of the vector are known. Figure 3.3 shows this graphically. Equation (3.10) applies for solar radiation to the tram.

$$
\dot{Q}_{\mathrm{sol}}=k_{\mathrm{sol}} \overrightarrow{\dot{\mathbf{q}}}_{\text {sol }} \mathbf{O}_{\mathrm{sol}} \text {. }
$$


The values of $\overrightarrow{\mathbf{q}}_{\text {sol }}$ and $\mathbf{O}_{\text {sol }}$ follow after transforming the solar radiation vector into Cartesian coordinates. Equation (3.11) and Equation (3.12) show this transformation.

$$
\begin{aligned}
\overrightarrow{\mathbf{q}}_{\text {sol }} & =\left|\overrightarrow{\mathbf{q}}_{\text {sol }}\right|(|\cos (\xi) \sin (\zeta)| \quad|\sin (\xi) \sin (\zeta)||\cos (\zeta)|) \\
\mathbf{O}_{\text {sol }} & =\left(\begin{array}{l}
l_{\text {height }} \cdot l_{\text {width }} \\
l_{\text {length }} \cdot l_{\text {height }} \\
l_{\text {length }} \cdot l_{\text {width }}
\end{array}\right)
\end{aligned}
$$

The dimensions of the tram are: width $l_{\text {width }}=2.4 \mathrm{~m}$, height $l_{\text {height }}=3 \mathrm{~m}$, length for the $1^{\text {st }}$ and $3^{\text {rd }}$ section $l_{\text {length }}=9.29 \mathrm{~m}$, and length of the $2^{\text {nd }}$ section $l_{\text {length }}=5.63 \mathrm{~m}$

\section{Passengers}

EN 14750 part 1 [60, Appendix D] shows the amount of heat introduced by one passenger. This work assumes the total amount of waste heat by a person as independent of the temperature as

$$
\dot{q}_{\text {pas }}=120 \mathrm{~W}=\text { const. }
$$

The waste heat of a person consists of a sensible (increases the temperature) and a latent (increases the humidity) part. A piece-wise linear function (see Equation (3.14)) estimates the latent amount.

$$
\dot{q}_{\text {pas,lat }}\left(T_{\text {IDA }}\right)=\left\{\begin{array}{lr}
20 & T_{\text {IDA }}<18 \\
\frac{10}{3} T_{\text {IDA }}-40 & 18 \leq T_{\text {IDA }}<24 \\
5 T_{\text {IDA }}-80 & 24 \leq T_{\text {IDA }}<35 \\
95 & T_{\text {IDA }} \geq 35
\end{array}\right.
$$

This yields a sensible amount by a person of

$$
\dot{q}_{\text {pas,sen }}=\dot{q}_{\text {pas }}-\dot{q}_{\text {pas,lat }} .
$$

The equations do not consider the effect of a person's activity (e.g. standing or sitting) on heat emission. The total heat emitted by passengers follows from multiplying the specific heat flow by the number of passengers.

$$
\dot{Q}_{\text {pas,sen }}=\dot{q}_{\text {pas,sen }} \cdot n_{\text {pas }} \text {, }
$$

in which $n_{\mathrm{pas}}$ is the number of passengers. Remark: Equation (3.1) applies only the sensible part $\dot{Q}_{\text {pas,sen }}$ for the temperature calculation.

\section{Electric lighting}

The installed electric lighting emits heat into the tram

$$
\dot{Q}_{\text {light }}=\delta_{\text {light }} \cdot n_{\text {light }} \cdot \dot{q}_{\text {light }} \text {, }
$$


where $n_{\text {light }}$ is the number of lamps and $\dot{q}_{\text {light }}$ the electric power by a lamp. $\delta_{\text {light }}$ shows the state of lighting. It is

$$
\delta_{\text {light }}=\left\{\begin{array}{ll}
1 & \text { On } \\
0 & \text { Off }
\end{array} .\right.
$$

\subsubsection{Mass flows}

The mass flows are split by their type, into mass flows

- of dry air,

- of water and

- of $\mathrm{CO}_{2}$.

The following paragraphs explain them in detail.

Mass flows of (dry) air

Figure $3.2 \mathrm{~b}$ shows that mass flows of supply and exhaust air must be considered.

Supply air The heat flow that is introduced into the interior through the supply air is as follows

$$
\dot{E}_{\mathrm{SUP}}=\dot{m}_{\mathrm{SUP}} \cdot c_{\mathrm{air}} \cdot T_{\mathrm{SUP}}
$$

whereby $\dot{m}$ means the mass flow, $c$ the heat capacity and $T$ the temperature of supply air (SUP).

Exhaust air The heat flow leaving the tram as exhaust air is similar to Equation (3.19)

$$
\dot{E}_{\mathrm{EHA}}=\dot{m}_{\mathrm{SUP}} \cdot c_{\mathrm{air}} \cdot T_{\mathrm{IDA}} \cdot
$$

The mass flow of supplied air agrees with the mass flow of dissipated air (see Equation (3.5)). A distinction of mass flows into recirculation air (RCA) and exhausted air (EHA) is not necessary at this point because it is not important for the interior where the mass flow goes to.

\section{Mass flows of water}

The mass flows of water (superscript w) consists of supply air, exhaust air and the mass flow introduced by the passengers (e.g. sweat). The model does not consider extra water sources (e.g. rain) because they are not measurable.

Supply air For the supply air follows

$$
\dot{E}_{\mathrm{SUP}}^{\mathrm{W}}=\dot{m}_{\mathrm{SUP}} Y_{\mathrm{SUP}}
$$

Passengers The previous subsection splits the heat flow by the passengers into two parts: a sensible part that effects the temperature and a latent part. The latent part converts into a mass 
flow of water by dividing it by the heat of evaporation of water.

$$
\dot{E}_{\mathrm{pas}}^{\mathrm{W}}=\frac{\dot{q}_{\mathrm{pas}, \mathrm{lat}}}{\Delta h_{\mathrm{V}}} \cdot n_{\mathrm{pas}}
$$

Exhaust air For the exhaust air follows

$$
\dot{E}_{\mathrm{EHA}}^{\mathrm{W}}=\dot{m}_{\mathrm{SUP}} Y_{\mathrm{IDA}} .
$$

Equation (3.19) explains the assumptions for exhaust air. They are also valid here.

\section{Mass flows of carbon dioxide}

The mass flows of $\mathrm{CO}_{2}$ are calculated similarly to the mass flows of water. The section explains the supply air, exhaust air and the amount introduced by passengers.

Supply air For the supply air follows

$$
\dot{E}_{\mathrm{SUP}}^{\mathrm{CO} 2}=\dot{m}_{\mathrm{SUP}} \cdot c_{\mathrm{SUP}} \cdot
$$

Passengers For the passengers

$$
\dot{E}_{\mathrm{pas}}^{\mathrm{CO} 2}=k_{\mathrm{pas}, \mathrm{CO} 2} \cdot n_{\text {pas }}
$$

applies, in which $k_{\mathrm{pas}, \mathrm{CO} 2}$ describes how much $\mathrm{CO}_{2}$ each person emits.

Exhaust air For the exhaust air follows

$$
\dot{E}_{\mathrm{EHA}}^{\mathrm{CO} 2}=\dot{m}_{\mathrm{IDA}} \cdot \sigma_{\mathrm{IDA}} .
$$

\subsection{HVAC model}

The objective of the HVAC calculation results from the scope of the overall model. The model design is similar to the tram and is gained from the HVAC design. This section describes the HVAC parts by individual analytical models.

\section{Calculation objective}

The objective of the HVAC's calculation is to calculate the resulting heat flows, the resulting state of the supply air and the total electrical power. Inputs are the conditions of the tram, the manipulated variables (modes of the finite state machine (FSM), see Section 3.4) and the environmental conditions. 


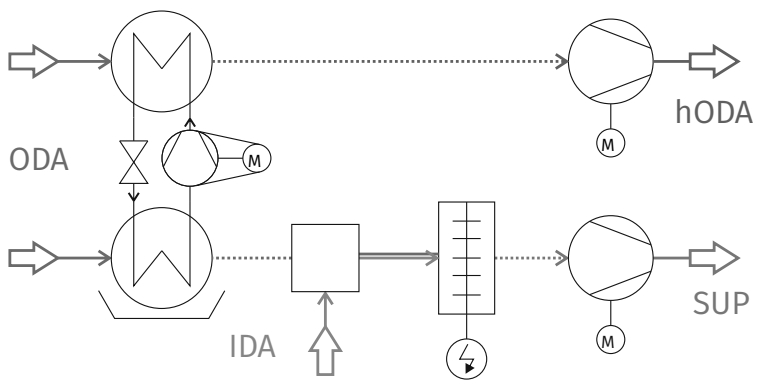

(a) Schematic diagram of the HVAC

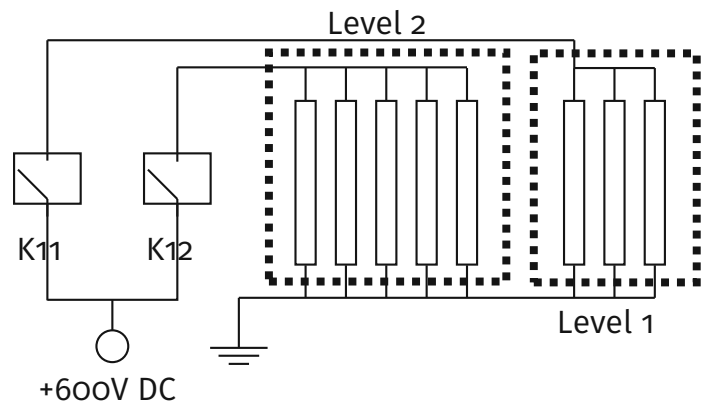

(b) Electrical cabling of the electric heater

Figure 3.4. - Schematic representation of the HVAC

\section{HVAC design}

Figure 3.4a shows a schematic diagram of the HVAC. It shows the individual parts of the HVAC by symbols and the mass flows by coloured arrows. The symbols are from [90, Annex C]. The HVAC sucks in the ODA (green, [88]) and cools it by the CRM (green dotted). Depending on the global mode of the FSM, the (cooled) ODA mixes (green-orange) with the indoor air (IDA) (orange). The electric heater heats the IDA (blue dotted) and blows it into the tram's interior by the supply air fan as SUP (blue). In addition, the cooling fan delivers a mass flow through the condenser of the CRM to spread the heat. The HVAC consists of the following parts (see Figure 3.4a)

- an electric heater,

- a CRM (incl. heat exchanger, compressor, choke and bypass),

- a mixing chamber and

- fan(s).

The following subsections describe the individual parts in detail.

\subsubsection{Electric heater}

The electric heater (also heating element) of the HVAC heats the mixing air (MIA), which in turn heats the interior of the vehicle as SUP. A non-stationary energy balance is stated for the electric heater. The differential equation is

$$
C_{\mathrm{eh}} \cdot \dot{T}_{\mathrm{eh}}=P_{\mathrm{eh}}-\dot{Q}_{\mathrm{eh}},
$$

in which $C$ describes the heat capacity, $P$ the supplied electrical power and $\dot{Q}$ the heat flow emitted by the electric heater. The following two paragraphs describe the calculation of the electrical power and the emitted heat flow. The weight of the electric heater and the used materials allow to estimate the heater's heat capacity.

\section{Electric calculations}

The manufacturer has designed the electric parts of the HVAC for $600 \mathrm{~V}$ direct-current voltage. Tolerance of the voltage is $-20 \%$ and $+30 \%$. Currently, the voltage of the catenary wire is $710 \mathrm{~V}$, 
Table 3.2. - Data of the electric heater at $25^{\circ} \mathrm{C}$

\begin{tabular}{lrrr}
\hline & \multicolumn{2}{c}{$P$ in W } & \multirow{2}{*}{ R in $\Omega$} \\
\cline { 2 - 3 } & $600 \mathrm{~V}$ & $710 \mathrm{~V}$ & \\
\hline 1 bar & - & - & $490.8 \Omega$ \\
level 1 & $7335 \mathrm{~W}$ & $10270 \mathrm{~W}$ & $49.08 \Omega$ \\
level 2 & $14670 \mathrm{~W}$ & $20540 \mathrm{~W}$ & $24.54 \Omega$ \\
level 3 & $22000 \mathrm{~W}$ & $30810 \mathrm{~W}$ & $16.36 \Omega$ \\
\hline
\end{tabular}

the modelling considers this deviation. The electrical power of a resistive load is

$$
P_{\mathrm{eh}}=\frac{U^{2}}{R}
$$

The electric heater is considered as such. $U$ is the supply voltage of the tram and $R$ is the electrical resistance. Equation (3.28) shows that the voltage affects the electrical power quadratically. Due to the deviation between design point and operation point, a potential for errors of $30 \%$ occurs for static modelling. The modelling therefore considers the electrical connection of the electric heater.

Equation (3.28) and the measured values in the climatic wind tunnel determine the wiring of the electric heater, as shown in Figure 3.4b. According to Equation (3.28), the electrical power is calculated as a function of setting and voltage. Table 3.2 lists the resistance of the settings as well as the resulting electrical power at $600 \mathrm{~V}$ and $710 \mathrm{~V}$.

\section{Air-side calculation}

The calculation of the emitted heat flow uses [101], which is dissipated to the MIA. For the heat flow follows

$$
\dot{Q}_{\mathrm{eh}}=k_{\mathrm{eh}} \cdot O_{\mathrm{eh}} \cdot \Delta T_{\mathrm{med}}
$$

Based on the design drawing of the electric heater [117], the necessary parameters (e. g. cross section, ribs number and area, etc.) are estimated for calculating the heat transfer coefficient and the surface $O . \Delta T_{\text {med }}$ is the thermodynamic medium temperature. Table A.6 (see Appendix A.2) lists the used parameters.

\subsubsection{Compression refrigeration machine}

Calculating the cooling power needs the pressures of the cooling cycles. This and other input variables (e.g. state of ODA and the states of the fans) are calculated for the air condition after the CRM is calculated (air-side calculation). Figure 3.5a shows the CRM with its parts in detail.

The next paragraph explains how to calculate the cooling cycle. 


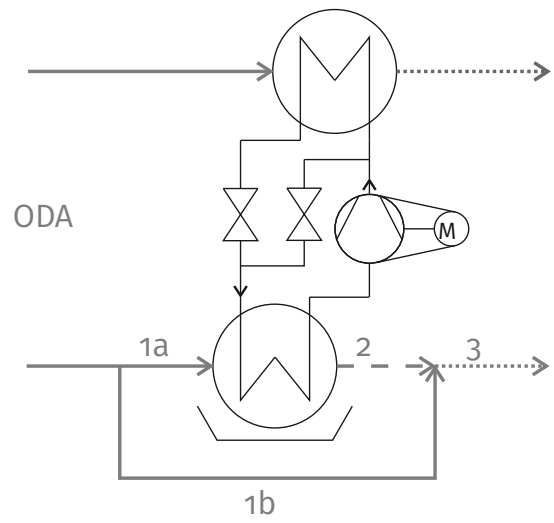

(a) Detailed sketch of the CRM

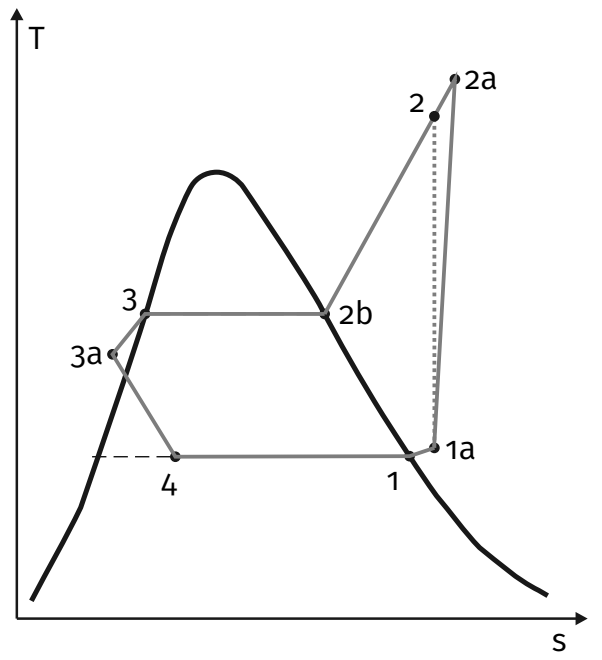

(b) T,S - diagram of the cooling cycle (schematic)

Figure 3.5. - CRM: detailed sketches

\section{Cooling cycle}

The cooling cycle is calculated according to a standard work of thermodynamics [5, p. 594ff]. The following paragraph describes the simplified states of the refrigerant and the state changes in the cooling cycle leading to these states. The numbering of states is identical with Figure 3.5b.

- State 1: The refrigerant is in a thermodynamic state known as saturated vapour.

- Change of state (CS) $1 \rightarrow$ 1a: The refrigerant is heated isobaric from state 1 to 1a (superheating).

- CS 1a $\rightarrow$ 2: An ideal reversible compressor would compress the refrigerant from state 1a to 2.

- CS 1a $\rightarrow$ 2a: However, the used irreversible compressor compresses the refrigerant from state 1 a to $2 a$.

- CS $2 \mathbf{a} \rightarrow \mathbf{2 b}$ : From the previous state, the refrigerant is cooled isobaric and starts to condense in state $2 \mathrm{~b}$.

- CS $\mathbf{2 b} \rightarrow \mathbf{b}$ : In state 3 the refrigerant is condensed completely (saturated liquid).

- CS $3 \rightarrow 3 a$ : The refrigerant is further cooled isobaric at the condensation line (subcooling).

- CS 3a $\rightarrow$ 4: From state 3a, the refrigerant undergoes a pressure drop and evaporates at the expansion valve.

- CS $4 \rightarrow 1$ : To close the cycle, the refrigerant is evaporated isobaric.

Empirical equations [7, S. 32] allow calculating the various states of the refrigerant. This approach simplifies a good generalisation for the refrigerant. The cited literature contains equations for the refrigerants of ammonia, R 22, R 152a, R 134a and R 123.

The compressor's data sheet [22] specifies its mass flow $\left(\dot{m}_{\mathrm{KM}}\right)$. This results in the absorbed heat 
flow $\dot{Q}_{\mathrm{KM}, \text { cool }}$, the rejected heat flow $\dot{Q}_{\mathrm{KM} \text {,exheat }}$, and the consumed power of the compressor $P_{\mathrm{KM}}$

$$
\begin{aligned}
\dot{Q}_{\mathrm{KM}, \text { cool }} & =\dot{m}_{\mathrm{KM}} \cdot\left(h_{1 a}-h_{4}\right) \\
\dot{Q}_{\mathrm{KM}, \text { exheat }} & =\dot{m}_{\mathrm{KM}} \cdot\left(h_{2 a}-h_{3 a}\right) \\
P_{\mathrm{KM}} & =\dot{m}_{\mathrm{KM}} \cdot\left(h_{2 a}-h_{1 a}\right) .
\end{aligned}
$$

To achieve better matching between simulation and measurement, the characteristic diagram of the compressor was adapted, which was necessary to meet the various uncertainties made during the modelling of the cooling cycle. This correction includes various doubts (e.g. compressor efficiency) and assumptions (e.g. subcooling temperature). For the compensated mass flow of the compressor follows

$$
\dot{m}_{\mathrm{KM}}=\dot{m}_{\mathrm{KM}}\left(T_{\text {evap }}, T_{\text {cond }}\right)+\dot{m}_{\mathrm{KM}, \text { corr }},
$$

in which $T_{\text {evap }}$ is the evaporation temperature and $T_{\text {cond }}$ the condensation temperature of the refrigerant. The correcting summand was estimated as a function of the bypass valve by the least squares method to better consider the effect of the bypass. Table A.7 (see Appendix A.2) lists the assumptions of the cooling cycle.

The author found out during modelling that the bypass valve affects the energy consumption. Especially at low outdoor air temperatures, the deviation is big. Currently, there is no information about the exact effect of the bypass valve available.

\section{Air-side calculation}

The mass flow of ODA, which enters the CRM (see Figure 3.5a), splits into two subflows. One subflow (1a) enters the CRM. This subflow cools down to the dew point. If the available cooling power is bigger than the absorbed energy, water is removed from the subflow 1a by condensation $(\varphi=1)$ until the whole cooling power is used (subflow 2). The authors of [122] describe this approach. The cooled and dehumidified subflow 2 mixes adiabatically with the bypassed subflow $1 b$, which results in subflow 3 .

The cooling air fan produces a mass flow and the calculation uses this mass flow of the cooling air (COA) (see Figure 3.4a). The fan sucks this mass flow through the condenser and the ODA absorbs the waste heat of the CRM. The cooling air fan spreads the COA to the environment, it is (currently) not used for further calculations. This approach allows simulating a heat pump with moderate changes to the model.

\subsubsection{Mixing chamber}

The mixing chamber mixes the two air flows isobaric (1a, 1b) if the chamber is in a working state. Otherwise, the mixing air equals (cooled) outdoor air. 


\begin{tabular}{ccccc} 
Table 3.3. - Volume flow of the supply air fan in $\mathrm{m}^{3} \mathrm{~h}^{-1}$ \\
\cline { 2 - 5 } & M1 & M2 & M3 & M4 \\
\hline Heating 1 & 440 & 330 & 340 & 375 \\
Heating 2 & 660 & 440 & 450 & 515 \\
Heating 3 & 800 & - & 620 & - \\
Cooling 1 & 950 & - & 850 & 965 \\
Cooling 2 & 1245 & 1230 & 1150 & 1260 \\
Cooling 3 & 1630 & 1650 & 1550 & 1740 \\
\hline
\end{tabular}

\subsubsection{Fans}

The fans produce a volume flow $\dot{V}_{\text {meas }}$ by overcoming the back pressure as a function of the supply voltage.

\section{Supply air fan}

The aim of the analytical calculation is to find one (more or less) consistent back pressure for the global modes of Heating and Cooling. The author did not find a valid back pressure with the known characteristic curve of the supply air. This is because the air ducts in Heating and Cooling are too different.

The M3 measurements of the volume flow were the basis for a data-driven adaptation. Table 3.3 lists various measurements of the volume flow. A correcting factor $\dot{V}_{\text {corr }}=1.1$ adapts the simulations to the measurements. The model is very sensitive to changes in the volume flow because the volume flow of the HVAC has a big impact on the tram model (see Equation (3.19) and Equation (3.20)). For the corrected volume

$$
\dot{V}_{\text {SUP }}=\dot{V}_{\text {corr }} \cdot \dot{V}_{\text {meas }}
$$

applies. Measurement data allow to discover the power consumption for individual levels.

\section{Cooling air fan}

The author realised the cooling air fan according to the statements of the manufacturer.

\subsection{Controller model of the current controller}

This section models the currently used controller. The figures given below are schematic representations of various FSM. The ellipses contain the mode of the FSM. The arrows between the ellipses depict the switching conditions of the FSM and the label is Bxx, in which $\mathrm{xx}$ is a number. The mode of the FSM will change if a switching condition becomes logically true. If no switching condition is true, the FSM will stay in the current mode. Appendix A.1 lists the switching conditions. 


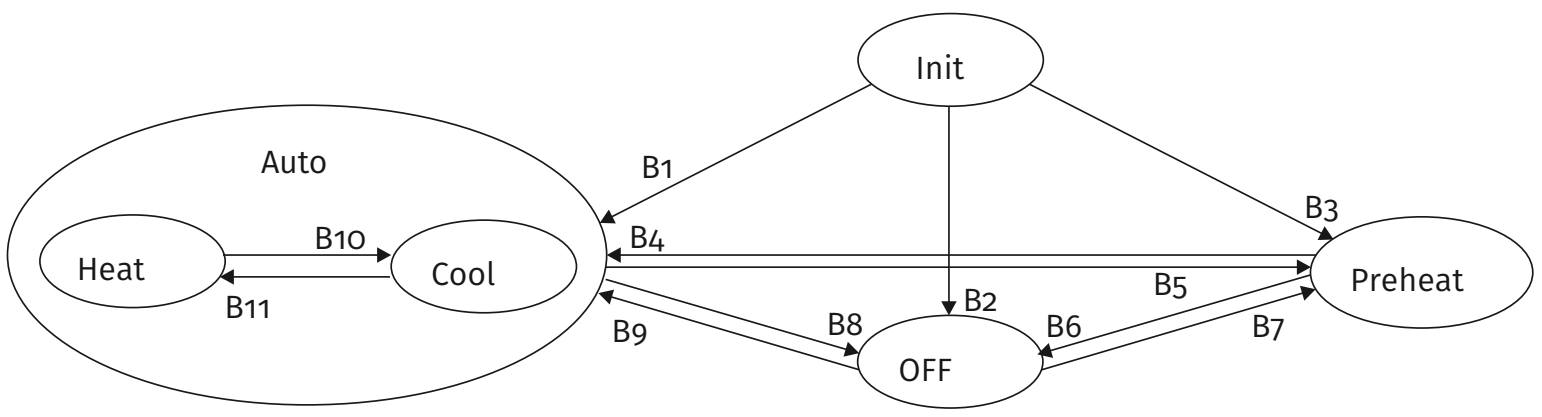

Figure 3.6. - Schematic diagram of the global FSM of the HVAC

The controller is implemented as FSM, in which the individual parts of the HVAC are FSMs for themselves. However, the switching conditions of the part's FSM depend on the global FSM. The following subsections explain the individual parts of the FSM, comprising

- Global FSM,

- Electric heater,

- Compressor,

- Bypass valve,

- Supply air fan and

- Cooling air fan.

\subsubsection{Global FSM}

Figure 3.6 shows the global FSM of the HVAC. There is not enough space in the illustrations to write out the switching conditions, so that the figures only show abbreviations. Various tables of Appendix A.1 list all the switching conditions. Table A.1 shows the switching conditions of the global FSM. The driver manually chooses the mode of the HVAC by a switch: Off, Preheating or Auto. Adding an Init mode simplifies the modelling because it allows simulating the FSM to start from a defined starting point. In Auto mode, extra switching conditions allow controlling the chosen mode (Heating or Cooling).

The global mode affects the mode of the mixing chamber (recirculation air On/Off). In the global Heating mode the recirculation air is On and in Cooling the recirculation air is Off. The mode of the mixing chamber changes directly by changing the global mode.

\subsubsection{Electric heater}

Figure 3.7a pictures an FSM with five modes. Table 3.2 lists three levels (1, 2 and 3), these levels agree with the FSM modes 1, 2 and 3. Depending on the mode of the global FSM, the switching conditions of the FSM of the electric heater change. Of course, it is not possible to use the electric heater during the global mode of Cooling - the mode of the electric heater is then always switched Off. It is not possible to use mode 3 of the electric heater during Preheating because electrical constraints of the catenary apply. (The driver gives the tram time to preheat itself before it is put in regular service. Usually, several trains enter service at a given time. So, generally, several trains 


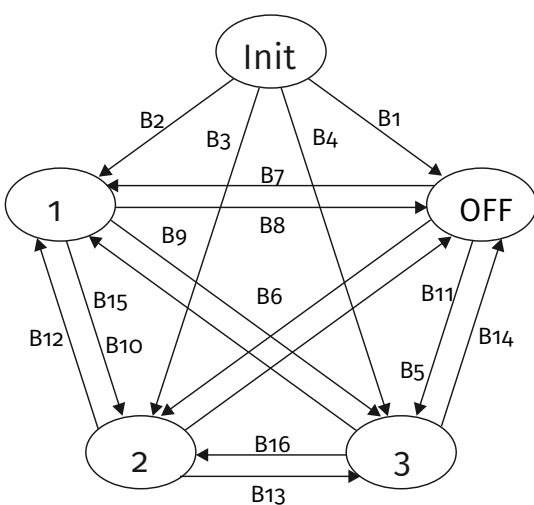

(a) FSM with five modes, e.g. the electric(b) heater

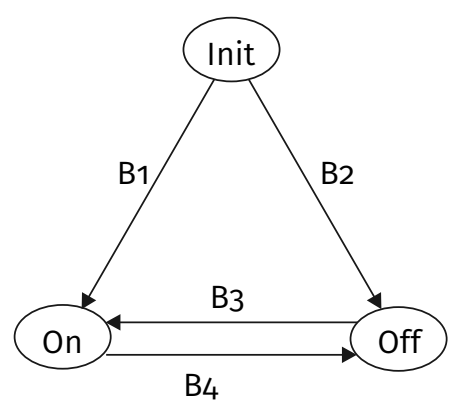

compressor

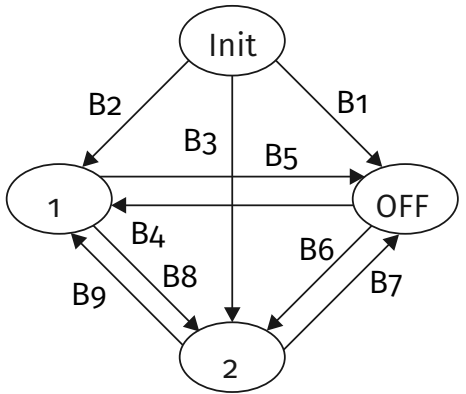

(c) FSM with four modes, e.g. of the fan

Figure 3.7. - Schematic diagrams of various finite state machine

preheat in parallel, As preheating the cooled down trains needs a high continuous power, which would overload the catenary, especially when several trains are preheated at the same time. As this is normally the case, the heating performance during preheating has been regulated. Table A.2 (see Appendix A.1) lists the switching conditions.

\subsubsection{Compressor}

The compressor is a part of the CRM. Figure 3.7b shows the FSM of the compressor with its switching conditions The mode changes only between On and Off. Table A.4 (see Appendix A.1) lists the switching conditions.

\subsubsection{Bypass valve}

The bypass valve prevents icing of the evaporator heat exchanger of the CRM (see Section 3.3.2). Although the flow is continuously controlled by the valve, the FSM has only two modes: On and Off. The HVAC's controller only switches between On and Off. The exact coolant mass flow results from the control of the bypass valve, which is realised in hardware. Figure 3.7b shows the FSM of the bypass valve with its switching conditions. Table A.4 (see Appendix A.1) lists the switching conditions.

\subsubsection{Supply air fan}

The supply air fan blows a volume flow through the HVAC (see Section 3.3.4). The fan has three levels. Figure 3.7a graphically explains the FSM of the supply air fan. Table A.5 (see Appendix A.1) lists the switching conditions. 


\subsubsection{Cooling air fan}

The cooling air fan removes heat from the condenser of the CRM, it has two levels. Figure 3.7c sketches the FSM of the cooling air fan. 


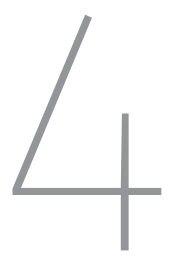

\section{Grey-Box Modelling}

This chapter exemplarily explains the grey-box modelling of several parts of the overall system. It discusses estimating the heat capacity and heat transfer coefficient in detail. Some parts of the HVAC are modelled analytically, like the electric heater discussed in Chapter 3, other parts are discussed here. For the controller of the HVAC, a current or true set point curve is estimated based on all available measurement data.

\subsection{Heat capacity and heat transfer coefficient of the tram model}

This section describes in detail estimating parameters with the help of the heat capacities and the $\mathrm{k}$-value. Further estimated parameters, such as the influence of solar radiation and the heat transfer through open doors, are briefly discussed afterwards. This section and the matching section in Section 5.5.2 were published as [56].

\subsubsection{Introduction}

Rail vehicles are the back bone of short-distance public transport (SDPT). In a large city like Vienna, the percentage of SDPT (including buses) is already at $35 \%$ [10]. As a result, the local operator serves, after own states, 2.5 million passengers per day [120].

Like every other industrial sector, the rail vehicle industry is subject to constant change. Since the 1950s one of the key trends is the occurring urbanisation which leads to a growth of cities and urban centres. By $205084 \%$ of the European population will live in urban areas [33].

As a result, the requirements of the population for mobility will increase in number and complexity. In order to sustain this growth, transport should mainly be covered by SDPT. The increased energy costs and the effort to reduce $\mathrm{CO}_{2}$ emissions support the use of rail vehicles. Thus, Vienna clearly prioritises public transport, walking and cycling over car traffic [99, p. 25].

In his seminal work Struckl [106] made a life cycle analysis of the metro Oslo to demonstrate energy saving potential for rail vehicles. About $30 \%$ of the total energy consumed is used for temperature control of the vehicle during driving cycles of the considered metro [106, see Fig. 4.53]. This magnitude is confirmed in [63]. In [93] it was measured that $11 \%$ of the total energy is 
consumed during stabling hours. The significant energy consumption of HVAC systems calls for an efficient design of the related components. A fundamental element of this task is a dynamic thermal model of rail vehicles.

For the calculation of the annual energy consumption of the HVAC [97] or other optimisation measures $[2,12]$, a mathematical description of the considered system needs to be found and parameters of this system need to be identified.

\section{Literature}

Vehicles are usually air-conditioned by vapour compression refrigerators, although other methods exist [18] and are applied in practise [124]. According to [1], there are three possibilities to model HVAC systems: physics based, data driven or grey box. The very same principles can be applied to thermal models of (rail) vehicles.

Marcos et al. [84] developed and validated an analytical (physics based) model of a car with two heat capacities. In [108] an analytical thermal model for an automotive vehicle is developed and validated. Parameters were estimated analytically (physics based), e.g. the heat capacity of the car was multiplied by the mass and the average specific heat capacity. Marachlian et al. [83] used an exergy based numerical model to simulate the cars HVAC operations. Today a thermal simulation software for automotive vehicles is already available [82] and used for the simulation of combined cooling loops [100]. The thermal behaviour of buildings can be simulated using a modular Modelica library [37].

In [77] dynamic cooling loads were studied using an analytical thermal model of a Chinese mainline train. Another dynamic thermal model of an urban rail vehicle is part of previous works of some of the authors and can be found in [54]. An analytical simulation model was also used by Li and Sun [76] to simulate and analyse the entire system of air conditioning unit and passenger room. All thermal vehicle simulation approaches are almost exclusively used for only one specific vehicle. The models that use an analytical approach are purposeful, but it is time consuming to determine every necessary parameter. For industrial practices, it would be advantageous if benchmarks or reference values for a dynamic thermal simulation model were available. Measurement data should be sufficiently available, because thermal tests of rail vehicles can look back on a long history,

Function tests have been conducted for rail vehicles in Vienna since 1958. Especially refrigerated wagons were examined back then and their heat transfer coefficient ( $k$ value) was measured. Over the years testing was expanded to passenger trains and metros. Nowadays function tests in a climatic wind tunnel (CWT) are part of standard commissioning. Since these tests are considered during construction of the vehicle, lower $k$ values are intended. The $k$ value of the vehicles is usually measured during these tests [44], according to European Standards [23, 24]. These tests are done to keep operational costs low and availability high [41, 45]. Although unpublished records about the identification of the heat capacity can be traced back until the 60s, no generally accepted procedures or regulations exists for the estimation.

In building technologies an obligatory holistic examination of buildings, in terms of energy efficiency, was introduced [25] by a new EU directive [35] and national law [115]. However, no standards exist for model structure and parameter choice of dynamic thermal models of rail vehicles' eigen behaviour. This paper is a step forward and provides a compact yet versatile model structure and reference values of the thermal behaviour of the vehicle for different analytical-thermal models and for various types of rail vehicles (tram, metro, regional and main-line). 


\section{Contents}

Different models that are describing the vehicles' behaviour are derived from a general dynamic model in Section 4.1.2. Afterwards the theoretical fundamentals and the practical requirements for the conducted and available measurements are discussed in Section 4.1.3. All assumptions are summarised in Section 4.1.4, the used simulation model and the estimation method are described in Section 4.1.5. In Section 6.2 the obtained results are shown and discussed.

\subsubsection{Modelling}

The modelling section summarises the state of art for thermal modelling for rail vehicles and presents a low-order dynamic model structure which can be efficiently parametrised.

\section{Generic analytic-dynamic thermal model}

Relevant variables for the thermal comfort inside the a rail vehicle are $T_{\text {IDA }}$ the temperature of the indoor air and $Y_{\text {IDA }}$ the absolute humidity of the indoor air, according to the current standards $[23,24]$. The air velocity is just defined as a constraint that must be satisfied and is usually only considered during design of the air distribution system.

The entire rail vehicle consists of $N$ thermal system. Every system $i$ of the rail vehicle can be described individually. Equations for $T_{\text {IDA }}$ and $Y_{\text {IDA }}$ can be stated based on energy and mass balances for every system $i$.

$$
\begin{aligned}
C_{i} \cdot \frac{\mathrm{d} T_{\mathrm{IDA}, i}}{\mathrm{~d} t} & =-\dot{Q}_{\mathrm{loss}, i}-\dot{Q}_{i, i+1}+\dot{Q}_{\mathrm{sol}, i}+\dot{Q}_{\mathrm{pas}, i}+\dot{Q}_{\mathrm{aux}, i}+\dot{E}_{\mathrm{SUP}, i}-\dot{E}_{\mathrm{EHA}, i} \\
m_{i} \cdot \frac{\mathrm{d} Y_{i}}{\mathrm{~d} t} & =\dot{E}_{\mathrm{SUP}, i}^{\mathrm{W}}-\dot{E}_{\mathrm{EHA}, i}^{\mathrm{W}}+\dot{E}_{\mathrm{pas}, i}^{\mathrm{W}}+\dot{E}_{\mathrm{aux}, i}^{\mathrm{W}}
\end{aligned}
$$

In Equation (4.1), $C_{i}$ is the heat capacity associated with temperature $T_{\text {IDAi }} \cdot \dot{Q}_{\text {loss }, i}$ describes the dissipated heat from the component of the system with the temperature $T_{i}$ to the environment. $\dot{Q}_{i, i+1}$ is the energy flow between the components with temperatures $T_{\mathrm{IDA}, i}$ and $T_{\mathrm{IDA}, i+1}$, respectively. $\dot{Q}_{\mathrm{sol}, i}$ takes the solar radiation (direct and indirect) and $\dot{Q}_{\mathrm{pas}, i}$ the dissipated sensible heat by passengers into account. $\dot{Q}_{\mathrm{aux}, i}$ is the heat dissipated by auxiliaries, i. e. electrical lighting and passenger information system. $\dot{E}_{\mathrm{SUP}, i}$ is the mass flow of supply air and $\dot{E}_{\mathrm{EHA}, i}$ the mass flow of exhaust air. In Equation (4.2) $m_{i}$ denotes the mass of the indoor air, $\dot{E}_{\mathrm{SUP}, i}^{\mathrm{W}}$ is the mass flow of water in the supply air and $\dot{E}_{\mathrm{EHA}, i}^{\mathrm{W}}$ in the exhaust air, $\dot{E}_{\mathrm{pas}, i}^{\mathrm{W}}$ is the dissipated water due to sensible heat of passengers and $\dot{E}_{\mathrm{aux}, i}^{\mathrm{W}}$ is any further mass flow of water in Equation (4.2).

Only parameters $C_{i}, m_{i}$ and terms $\dot{Q}_{\text {loss }, i}$ and $\dot{Q}_{i, i+1}$ are necessary to describe the vehicles' behaviour. Since the initial analytical guess of $m_{i}$, sufficed for the calculations of the absolute humidity $Y$ in previous works of some of the authors [28], this paper will focus on $C_{i}$ and the terms $\dot{Q}_{\text {loss }, i}$ and $\dot{Q}_{i, i+1}$ for the calculations of the temperature(s) $T_{\text {IDA }, i}$. The air volume of system $i$ was multiplied by the air density to obtain the mass of indoor air $m_{i}$.

Following subsections derive three vehicle models from the generic analytic-dynamic thermal model to describe the vehicles' behaviour. 


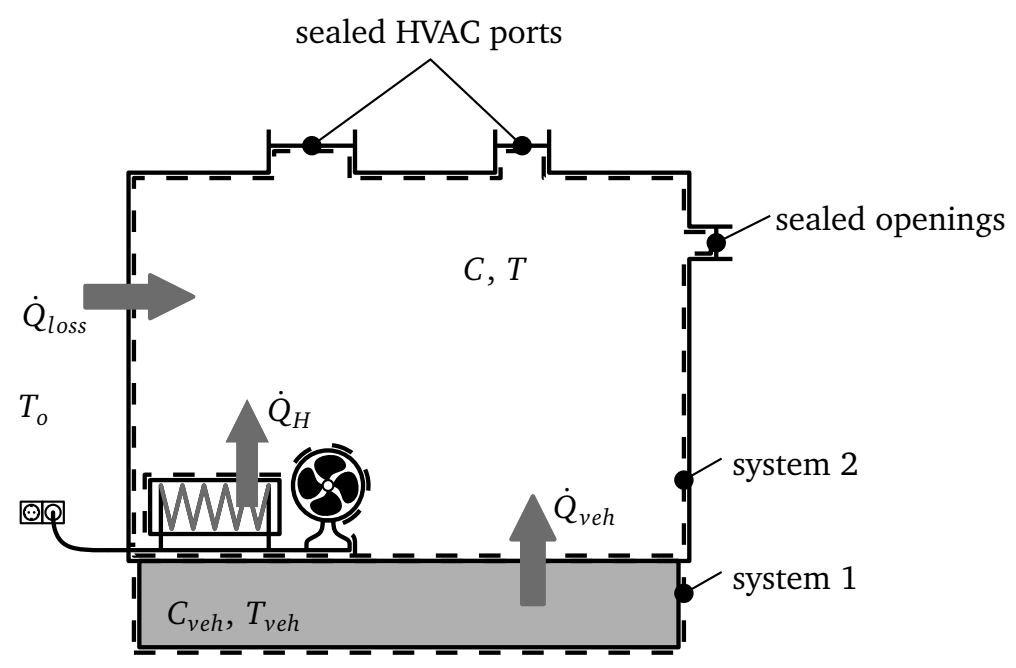

Figure 4.1. - Schematic illustration of the second-order thermal vehicle model

\section{Second-order thermal vehicle model}

The vehicle is assumed to consist of two systems $(N=2)$ with two heat capacities and two temperatures, see Figure 4.1. The equation for system $1(i=1)$ follows from Equation (4.1) by keeping only terms relevant to the vehicles' behaviour.

$$
C_{1} \cdot \frac{\mathrm{d} T_{\mathrm{IDA}, 1}}{\mathrm{~d} t}=-\dot{Q}_{\mathrm{loss}, 1}-\dot{Q}_{1,2}+\dot{Q}_{\mathrm{sol}, 1}+\dot{Q}_{\mathrm{pas}, 1}+\dot{Q}_{\mathrm{aux}, 1}+\underbrace{\dot{E}_{\mathrm{SUP}, 1}-\dot{E}_{\mathrm{EHA}, 1}}_{\dot{Q}_{\mathrm{tot}}}
$$

In Figure 4.1 it is shown that HVAC ports are sealed. So, $\dot{E}_{\mathrm{SUP}, 1}$ and $\dot{E}_{\mathrm{EHA}, 1}$ need to be replaced by $\dot{Q}_{\text {tot }}$ during measurements (see Section 4.1.3) which holds the sum of all introduced heat flows. Equation (4.3) is obtained by renaming $\dot{Q}_{1,2}$ to $\dot{Q}_{\mathrm{veh}}$ and omitting index 1 .

$$
C_{\mathrm{IDA}} \cdot \frac{\mathrm{d} T_{\mathrm{IDA}}}{\mathrm{d} t}=\dot{Q}_{\mathrm{tot}}-\dot{Q}_{\mathrm{loss}}-\dot{\mathrm{Q}}_{\mathrm{veh}}
$$

in which $T_{\text {IDA }}$ is the indoor air temperature and $C_{\text {IDA }}$ the heat capacity of the interior. $\dot{Q}_{\text {tot }}$ is the sum of all introduced heat flows, $\dot{Q}_{\text {loss }}$ indicates the emitted heat flow to the environment and $\dot{Q}_{\text {veh }}$ is the heat flow to the car body (system 2).

For system 2 the described steps for system 1 are repeated. Additionally, it was assumed that $\dot{Q}_{\text {loss }, 2}$ is zero for system 2 . With this design choice the model stays compatible to the current standardised model, see Section 4.1.2, and all (analytical) knowledge for the $k$ value is sustained. Then, $C_{2}$ is renamed to $C_{\text {veh }}$ and $T_{\mathrm{IDA}, 2}$ is renamed to $T_{\mathrm{veh}}$. The temperature of the vehicle body $T_{\text {veh }}$ is calculated using

$$
C_{\mathrm{veh}} \cdot \frac{\mathrm{d} T_{\mathrm{veh}}}{\mathrm{d} t}=\dot{Q}_{\mathrm{veh}}
$$


$C_{\text {veh }}$ is the heat capacity of the vehicle body. Heat flow through surface $O$ is assumed as heat flow through a flat vertical wall $\dot{Q}_{\text {loss }}$, in which

$$
\dot{Q}_{\text {loss }}=k \cdot O \cdot\left(T_{\mathrm{IDA}}-T_{\mathrm{ODA}}\right)
$$

applies. $O$ is the overall uncoiled surface of the vehicle. $k$ is the heat transfer coefficient and $T_{\mathrm{ODA}}$ is the outdoor air temperature.

To complete the model, the heat transfer between the interior room (system 1) and the capacity (system 2) is described as

$$
\dot{Q}_{\mathrm{veh}}=k_{\mathrm{veh}} \cdot\left(T_{\mathrm{IDA}}-T_{\mathrm{veh}}\right) .
$$

$k_{\mathrm{veh}}$ is the heat transfer coefficient between the interior and the surface of the modelled heat capacity of the vehicle body $C_{\text {veh. }}$. However, a split-up into two factors, as done in Equation (4.5), is not reasonable since neither the surface nor the heat transfer coefficient can be chosen distinctively. It is important to note, that $C_{\text {veh }}, k_{\text {veh }}$, and $T_{\text {veh }}$ are purely fictitious quantities, and a strictly physical interpretation is not possible.

The two heat capacities ( $C_{\text {IDA }}$ and $C_{\text {veh }}$ ) can be interpreted in the following way: The heat capacity $C_{\text {IDA }}$ not only consists of the heat capacity of the indoor air, but also of the heat capacity of those parts of the passenger cabin which are in thermal equilibrium with the indoor air (e.g. seats, passenger handles, etc.). The heat capacity of the air inside the interior alone is not large enough to describe the dynamic behaviour adequately. Consequently, the heat capacity $C_{\text {veh }}$ consists of those parts of the vehicle that exchange heat with the heat capacity $C_{\text {IDA }}$.

The equations of the second-order model can be summarised to an equation for the indoor air temperature $T_{\text {IDA }}$ and an equation for the vehicle temperature $T_{\text {veh }}$ :

$$
\begin{aligned}
& C_{\mathrm{IDA}} \cdot \frac{\mathrm{d} T_{\mathrm{IDA}}}{\mathrm{d} t}=\dot{Q}_{\mathrm{tot}}-k \cdot O \cdot\left(T_{\mathrm{IDA}}-T_{\mathrm{ODA}}\right)-k_{\mathrm{veh}} \cdot\left(T_{\mathrm{IDA}}-T_{\mathrm{veh}}\right), \\
& C_{\mathrm{veh}} \cdot \frac{\mathrm{d} T_{\mathrm{veh}}}{\mathrm{d} t}=k_{\mathrm{veh}} \cdot\left(T_{\mathrm{IDA}}-T_{\mathrm{veh}}\right) .
\end{aligned}
$$

A schematic illustration of the second-order thermal vehicle model can be found in Figure 4.1.

\section{First-order thermal vehicle model}

In this model the vehicles' behaviour is described by only one system. In addition to assumptions made in Section 4.1.2, $\dot{Q}_{\text {veh }}$ is also assumed to be zero. Hence,

$$
\bar{C} \frac{\mathrm{d} T_{\text {IDA }}}{\mathrm{d} t}=\dot{Q}_{\mathrm{tot}}-k \cdot O \cdot\left(T_{\mathrm{IDA}}-T_{\mathrm{ODA}}\right)
$$

can be obtained, in which $\bar{C}$ is the heat capacity of the vehicle. 


\section{Standardised static model}

The static model can be obtained by introducing the additional assumption that $\frac{d T_{\mathrm{IDA}}}{d t}=0$ to the already made assumptions in Section 4.1.2. As a result the energy balance simplifies to

$$
0=\dot{Q}_{\mathrm{tot}}-k \cdot O \cdot\left(T_{\mathrm{IDA}}-T_{\mathrm{ODA}}\right),
$$

resulting in the standardised model $[23,24]$.

It is important to note that both proposed dynamic models are consistent with the standardised static model.

Equation (4.3) may be derived from the total energy balance (assuming that the temperature variations in the rail vehicle are small and that the specific heat capacity is constant). However, it is beyond the scope of this paper to show mathematically that the second law of thermodynamics is always guaranteed for the presented models. But since Equation (4.3) is frequently used, the standard model is applied in this study [20, cf. Example 11.4].

\subsubsection{Measurements}

Parameters of the proposed models are identified from measured data. First, general requirements for these measurements must be specified. In addition, two measurement approaches can be distinguished:

- Special experiments (step responses)

- Existing experiments (data from standard commissioning)

Requirements for measurement data of both approaches are described in this Section.

\section{General Requirements}

To estimate parameters $C_{\text {IDA }}, k, C_{\mathrm{veh}}, k_{\mathrm{veh}}$ of the model from CWT measurement data, measurements of the model inputs (sum of all introduced heat flows $\dot{Q}_{\text {tot }}$ and the temperature inside the CWT $T_{\text {ODA }}$ ) and model outputs (indoor air temperature $T_{\text {IDA }}$, vehicle temperature $T_{\text {veh }}$ ) are necessary. The indoor air temperature $T_{\text {IDA }}$ is considered to be the (weighted) average measurement of all temperature measurements inside of the vehicle. The vehicle temperature is also calculated as a split-up coverage from several measurements (see Section 4.1.4, Page 40). The sum of introduced heat flows $\dot{Q}_{\text {tot }}$ is calculated both from the absorbed electrical power of the heater coils and the absorbed electrical power of the fans.

Measurements need to be recorded with an adequately high sampling rate. The typically used sampling rate of $10 \mathrm{~s}$ to $1 \mathrm{~min}$ is sufficient. Since only the vehicles' behaviour should be measured, all other influences and disturbances, as shown in Equations (4.1) and (4.2), should be eliminated in the CWT as well as possible. The simulated wind speed should be kept constant, the simulated passenger load $\dot{Q}_{\text {pas }}$, the solar radiation $\dot{Q}_{\text {sol }}$ and additional auxiliaries $\dot{Q}_{\text {aux }}$ must be turned off. All openings $\dot{E}_{\mathrm{EHA}}$ and HVAC ports $\dot{E}_{\mathrm{SUP}}$ are sealed, as shown in Figure 4.1.

\section{Special Experiments}

Due to economical limitations, two step tests are conducted with different signs. Initial conditions should be well defined and known. Due to the fact, that the measurement of the vehicle temperature 


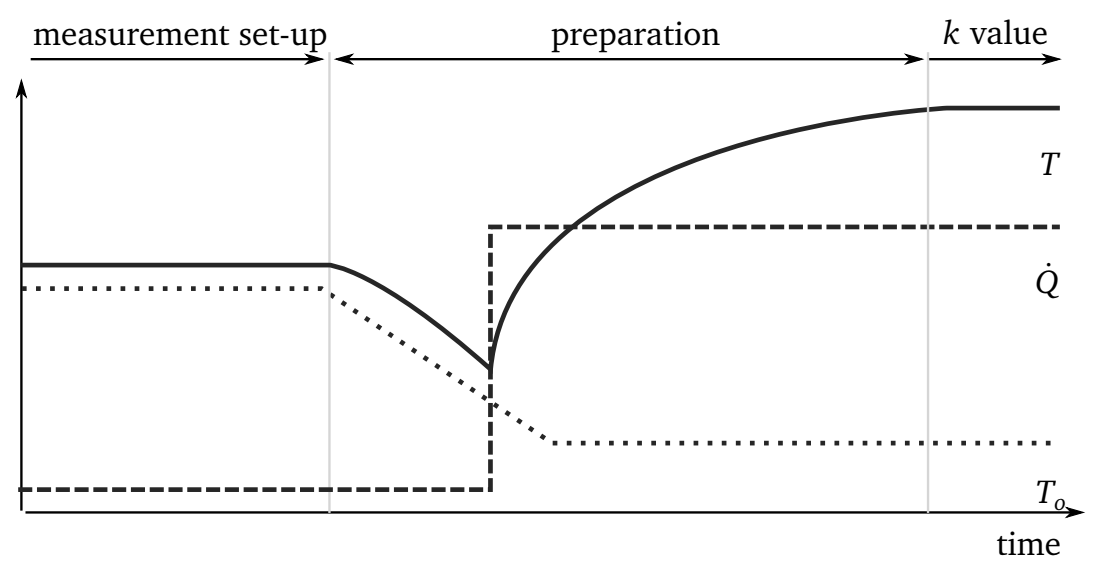

(a) Existing experiments

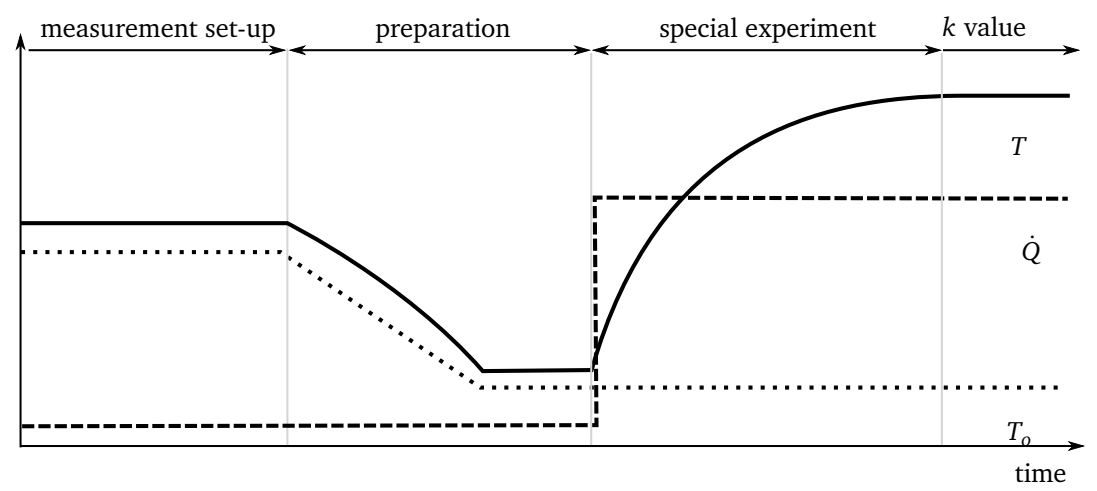

(b) Special experiments

Figure 4.2. - Chronological sequence of $x$ and $u$ for special and existing experiments (schematic diagram)

is difficult, it is desirable that the vehicle temperature $T_{\text {veh }}$ and the indoor air temperature $T_{\text {IDA }}$ are constant at the beginning of the experiment.

Based on this state, one input is changed stepwise. At time $t=0$ the supplied heat flow is switched to the value that follows from the expected $k$ value. Usually, a rough estimate is known, because it is analytically calculated by the manufacturer or a maximum permissible value can be found in the standards for the vehicle class $[23,24]$. The experiment is conducted until a new steady state follows, i. e. the derivative of the indoor air temperature $T_{\text {IDA }}$ and the deviations of the vehicle temperature $T_{\text {veh }}$ are almost zero. To reduce the necessary experiment time the experiment can be stopped earlier, if the final value can be estimated from measurement data and $95 \%$ of the step response has already been achieved. The step experiment is then repeated with reversed sign. After the experiment ends the initial conditions should be reached again.

\section{Existing measurements}

The procedure described for special experiments cannot be applied to historic measurements and they cannot be repeated at justifiable cost. However, in the conducted measurements similar 
sections to the proposed special experiments can usually be found. These can also be used for parameter estimation, but not with the same accuracy. This includes the following experiment types:

- Pre-heating experiments of the vehicle using HVAC (pre-heating)

- Measurement of the heat transfer coefficient ( $k$ value)

- Cool down experiment (Freezing test) The vehicle is allowed to cool down for $12 \mathrm{~h}$.

The pre-heating and cool down experiment have the disadvantage, that the introduced heat flow was not directly measured. Although it can be calculated from the measured air flow, the result is always subject to increased uncertainty. The measurement for estimating the heat transfer coefficient is almost always performed, though according to different standards.

This means that relevant parameters can typically be estimated with experiments for the measurement of the heat transfer coefficient, as well as the conducted preparation and post-processing experiments before and afterwards. Section 4.1.3 compares the existing measurements with the special experiments. In the special experiments only one input is changed at a time. First the temperature inside the CWT is set, a steady state is awaited and then $\dot{Q}_{\text {tot }}$ is changed.

\subsubsection{Assumptions}

The following assumptions have been made for the estimation of parameters:

- Ideal Mixing In the model it is assumed, that the air in the interior of the vehicle is ideally mixed. For the $k$ value experiments, this is ensured by temporary installed fans inside the vehicle.

- No heat transfer between outdoor air and heat capacity The heat transfer between outdoor air and modelled heat capacity of the vehicle is neglected. For all considered vehicles below this is satisfied.

- Measurement of the temperature of the vehicle's heat capacity As measurement value for the temperature of the vehicle's heat capacity the mean surface temperature of different surfaces inside the vehicle is used. All other considered metering points were not accessible for measurement.

- Identification An initial value must be given for identification. To estimate an accurate initial value a steady state is advantageous, unfortunately this state is not always available. Therefore, some experiments could not be used for identification (see Table 4.1).

- Linear Model

The proposed model for identification is linear in its parameters. Although non-linear behaviour is observed in measurement data, the linear model fit is considered satisfying.

The simulation model and the estimated parameters from the measured data are only valid, if these assumptions are met.

\subsubsection{Parameter estimation}

The dynamic models, described in Section 4.1.2, are transformed to an explicit linear state space representation. To ensure compliance with the current standardised method, the $k$ value is estimated 
Table 4.1. - Overview of the available and evaluated experiments for the estimation of heat capacities and heat transfer coefficient

\begin{tabular}{|c|c|c|c|c|c|c|}
\hline & క్ & $\begin{array}{l}\stackrel{0}{ \pm} \\
\stackrel{\Xi}{ \pm}\end{array}$ & 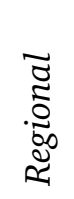 & 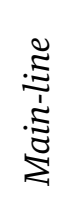 & 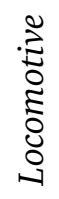 & $\underset{\omega}{\xi}$ \\
\hline$k$ value measured & 3 & 6 & 16 & 7 & 6 & 38 \\
\hline Experiment can be evaluated & 2 & 5 & 4 & 2 & 0 & 13 \\
\hline
\end{tabular}

accordingly. Afterwards, an assumption is made for the model structure by determining the noise model and the prediction error method (PEM) is used to estimate these parameters [78, 79].

\section{State-space representation}

A linear state space system is given by

$$
\begin{aligned}
& \dot{x}=\mathbf{A} x+\mathbf{B} u \text { and } \\
& y=\mathbf{C} x+\mathbf{D} u .
\end{aligned}
$$

The input vector $u$ is defined as $u^{T}=\left[\begin{array}{ll}T_{\text {ODA }} & \dot{Q}_{\text {tot }}\end{array}\right]$. C is always assumed to be the identity matrix of appropriate size and $\mathbf{D}=0$, so $y=x$.

For the first-order model $x=T_{\text {IDA }}$ is chosen, so

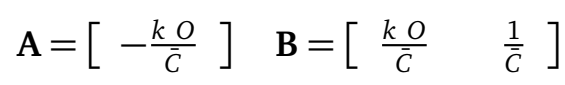

applies and for the second-order model $x^{T}=\left[\begin{array}{cc}T_{\text {IDA }} & T_{\text {veh }}\end{array}\right]$ is chosen, so

$$
\mathbf{A}=\left[\begin{array}{cc}
-\frac{k O+k_{\mathrm{veh}}}{C_{\mathrm{IDA}}} & \frac{k_{\mathrm{veh}}}{C_{\mathrm{IDA}}} \\
\frac{k_{\mathrm{veh}}}{C_{\mathrm{veh}}} & -\frac{k_{\mathrm{veh}}}{C_{\mathrm{veh}}}
\end{array}\right] \quad \mathbf{B}=\left[\begin{array}{cc}
\frac{k O}{C_{\mathrm{IDA}}} & \frac{1}{C_{\mathrm{IDA}}} \\
0 & 0
\end{array}\right]
$$

applies.

\section{Two-stage estimation approach}

To ensure compatibility with the current standardised static model, a two-stage approach is used for parameter estimation:

- Firstly, the $k$ value is estimated according to the standard method [24, p. 12]. Equations (4.11) and (4.12) are applied to appropriate measurement data.

- Secondly, missing parameters (first-order: $\bar{C}$ and second-order: $C_{\text {IDA }}, k_{\text {veh }}$ and $C_{\text {veh }}$ ) are additionally estimated using the PEM. 
Every system identification requires the assumption of a model structure, which is determined by the structure of the process and noise model. For linear systems the general linear discrete time state-space representation of a process and noise model is given by

$$
\begin{aligned}
x(n+1) & =\mathbf{A}(\boldsymbol{\theta}) \cdot x(n)+\mathbf{B}(\boldsymbol{\theta}) \cdot u(n)+\mathbf{K}(\boldsymbol{\theta}) \cdot \boldsymbol{e}(n) \\
y(n) & =\mathbf{C}(\boldsymbol{\theta}) \cdot x(n)+\mathbf{D}(\boldsymbol{\theta}) \cdot u(n)+\boldsymbol{e}(n)
\end{aligned}
$$

with the initial state vector $x(0)=x_{0}(\boldsymbol{\theta})$, the parameter vector $\boldsymbol{\theta}$ (cf. Equations (4.19) and (4.20)) and $\boldsymbol{e}(n)$ being a stochastic zero mean noise input [91]. The $\mathbf{K}$ matrix is fixed to zero (i. e. $\mathbf{K}=0$ ).

Using measurement data obtained by various experiments (see Section 4.1.3) in a CWT, parameters of the state-space system (cf. Equations (4.11) and (4.12)) can be identified by applying the PEM [78] according to the following criterion

$$
J(\boldsymbol{\theta})=\boldsymbol{e}^{\mathrm{T}} \boldsymbol{e} \longrightarrow \min _{\boldsymbol{\theta}},
$$

where

$$
\boldsymbol{e}=\left[\begin{array}{c}
\boldsymbol{\varepsilon}(1 \mid \boldsymbol{\theta}) \\
\vdots \\
\boldsymbol{\varepsilon}(N \mid \boldsymbol{\theta})
\end{array}\right] \text { with } \boldsymbol{\varepsilon}(n \mid \boldsymbol{\theta})=\boldsymbol{y}(n)-\hat{\boldsymbol{y}}(n \mid \boldsymbol{\theta}) .
$$

In Equation (4.18) $\varepsilon(n \mid \theta)$ is the prediction error (vector) (at time step $n$ ), which is the difference between the measured output (vector) $\boldsymbol{y}(n)$ and the predicted output (vector) of the model $\hat{\boldsymbol{y}}(n \mid \boldsymbol{\theta})$ and $N$ is the number of training data samples. As $k$ was already estimated during the first stage, the parameter vector is defined as

$$
\boldsymbol{\theta}=\bar{C}
$$

for the first-order model and

$$
\boldsymbol{\theta}=\left[\begin{array}{lll}
k_{\mathrm{veh}} & C_{\mathrm{IDA}} & C_{\mathrm{veh}}
\end{array}\right]^{T}
$$

for the second-order model. Other frequently used methods for estimation are the different leastsquares techniques [71]. The PEM has some advantages. It is applicable to a wide variety of model structures and it handles closed loop data in a direct fashion. A drawback is that it is labour intensive and requires good initial parameter values [79].

The implementation of the System Identification Toolbox of MATLAB was used. To begin with, the model with estimable parameters was defined using using the function idgrey of MATLAB and afterwards, parameters of this model were estimated using the function pem [85]. As initial values $C_{\mathrm{IDA}}=120480 \mathrm{~J} / \mathrm{K}, C_{\mathrm{veh}}=500000 \mathrm{~J} / \mathrm{K}$ and $k_{\mathrm{veh}}=20 \mathrm{~J} / \mathrm{K}$ were used. The calculation is performed on a contemporary Desktop PC (Intel Core i7 $860 @ 2.80 \mathrm{GHz}$ ) in a few seconds.

Table 4.2 lists the results of the identification. These estimated parameters were used to produce comparable time series plots of measurements and simulation (see Section 6.2). 


\begin{tabular}{|c|c|c|c|c|c|}
\hline Name & $\begin{array}{c}k \\
\mathrm{~W} /\left(\mathrm{m}^{2} \mathrm{~K}\right)\end{array}$ & $\begin{array}{l}C_{\text {IDA }} \\
\mathrm{J} / \mathrm{K}\end{array}$ & $\begin{array}{l}k_{\mathrm{veh}} \\
\mathrm{W} / \mathrm{K}\end{array}$ & $\begin{array}{l}C_{\mathrm{veh}} \\
\mathrm{J} / \mathrm{K}\end{array}$ & $\begin{array}{c}\mathbf{R}^{2} \\
1\end{array}$ \\
\hline Tram1 & 2.5 & $3.0 \cdot 10^{7}$ & 246 & $1.7 \cdot 10^{7}$ & 0.990 \\
\hline Tram2 & 3.2 & $1.8 \cdot 10^{7}$ & 974 & $1.0 \cdot 10^{7}$ & 0.995 \\
\hline Metro1 & 2.8 & $9.4 \cdot 10^{6}$ & 464 & $1.9 \cdot 10^{7}$ & 0.992 \\
\hline Metro2 & 3.1 & $9.2 \cdot 10^{6}$ & 199 & $9.6 \cdot 10^{6}$ & 0.949 \\
\hline Metro3 & 2.9 & $8.0 \cdot 10^{6}$ & 47,831 & $5.7 \cdot 10^{6}$ & 0.132 \\
\hline Metro4 & 2.4 & $4.6 \cdot 10^{6}$ & 230 & $1.3 \cdot 10^{7}$ & 0.628 \\
\hline Metro5 & 2.9 & $1.1 \cdot 10^{7}$ & 5,479 & $2.3 \cdot 10^{7}$ & 0.969 \\
\hline Regio1 & 1.7 & $4.3 \cdot 10^{7}$ & 484 & $4.6 \cdot 10^{6}$ & 0.820 \\
\hline Regio2 & 1.3 & $6.4 \cdot 10^{6}$ & 1,366 & $3.1 \cdot 10^{7}$ & 0.966 \\
\hline Regio3 & 2.0 & $3.6 \cdot 10^{6}$ & 217 & $1.1 \cdot 10^{7}$ & 0.126 \\
\hline Regio4 & 1.7 & $1.3 \cdot 10^{7}$ & 907 & $3.7 \cdot 10^{7}$ & 0.932 \\
\hline Main1 & 1.4 & $7.2 \cdot 10^{5}$ & 93,627 & $3.9 \cdot 10^{6}$ & 0.494 \\
\hline Main2 & 1.6 & $2.6 \cdot 10^{6}$ & 564 & $2.4 \cdot 10^{7}$ & 0.562 \\
\hline
\end{tabular}

Significance of estimated models

Let $y(n)$ denote the measured and $\hat{\boldsymbol{y}}(n)$ the simulated indoor air temperature at time step $n$ (for $n=1, \ldots, m)$, respectively. Then the coefficient of determination $\mathbf{R}^{2}$ of the model is given by

$$
\mathbf{R}^{2}=1-\frac{\mathrm{SS}_{\mathrm{res}}}{\mathrm{SS}_{\mathrm{tot}}}
$$

where $\mathrm{SS}_{\text {res }}$ is the sum of squares of residuals (also called the residual sum of squares) computed by

$$
\mathrm{SS}_{\mathrm{res}}=\sum_{n=1}^{m}(\underbrace{y(n)-\hat{\mathbf{y}}(n)}_{\boldsymbol{e}(n)})^{2}=\boldsymbol{e}^{\mathrm{T}} \boldsymbol{e}
$$

and $\mathrm{SS}_{\text {tot }}$ is the total sum of squares (proportional to the sample variance) obtained by

$$
\mathrm{SS}_{\mathrm{tot}}=\sum_{n=1}^{m}(y(n)-\bar{y})^{2},
$$

where $y=\frac{1}{m} \sum_{n=1}^{m} y(n)$ is the mean of the observed data [36].

The Coefficient of determination $\mathbf{R}^{2}$ is an indication that provides some information about the goodness of fit of a model. It describes how well measurements are reproduced by the thermal vehicle model. An $\mathbf{R}^{2}$ of 1 indicates that the simulated indoor air temperature perfectly fits the measured indoor air temperature. 


\subsection{Further parameters of the tram model}

\subsubsection{Solar radiation}

For current conditions inside the CWT $k_{\text {sol }}=0.27$ was determined using manual adjustment. This value agrees well with the magnitudes from [89].

\subsubsection{Passengers}

A constant heat dissipation by a passenger was assumed, as listed in Equation (3.13). This is justified for all CWT experiments, since the heat was introduced into the tram by heating mats and humidity by air humidifier. Further, it was assumed, that Equation (3.13), Equation (3.14) and Equation (3.16) are still valid for on-site experiments.

\subsubsection{Lighting}

There are 11 lamps $n_{\text {light }}$ with $36 \mathrm{~W}$ in each tram, according to verbal communication by Canori [19].

\subsubsection{Open doors}

Before passengers are able to get on or off the tram the doors have to open at a station. The open doors have an effect on the indoor air, because indoor air is replaced with outdoor air. Though the effect is generally known, it is hard to quantise. The effect lasts only for about 40-50 seconds, but indoor air temperature changes much slower. Explicit measurement, e.g. with an anemometer, is not possible during on-site operation of the tram due to security and vandalism concerns.

Although, the effect was observed by a higher energy consumption of the HVAC during on-site measurements compared to CWT tests. Therefore, a steady mass flow

$$
\dot{Q}_{\text {door }}=\delta_{\text {door }} \cdot \dot{m}_{\text {door }} \cdot c_{\text {air }} \cdot\left(T_{\mathrm{IDA}}-T_{\mathrm{ODA}}\right)
$$

is assumed during periods with open doors, in which $\delta_{\text {door }}$ represents the condition of the doors. 1 if the door is open, 0 otherwise.

The factor $\dot{m}_{\text {door }}$ was estimated using particle swarm optimisation. A value of $\dot{m}_{\text {door }}=0.0113 \mathrm{~kg} \mathrm{~s}^{-1}$ was found.

\subsection{HVAC model}

This section summarises estimated parameters for the HVAC model. 


\subsubsection{Electric heater}

In Section 3.3.1 an analytical modelling of the electric heater is described, which was used in the end. Used parameters were taken from data sheets or similar and are summarised in Table A.6.

In addition, several black box models were estimated by Euler-Rolle [32] as well. Because of the limited scope of models and not always given causality, the black box models were replaced by the described analytical model.

\subsubsection{CRM}

An airside calculation of the CRM needs only physical parameters listed in Table A.7. A satisfying modelling of the cooling cycle was only possible with extra measurements [118]. Correction summands were estimated for the refrigerant mass flow with and without operational bypass valve using least squares estimation (see Table A.7). Various non-linear approaches with matching estimated methods, such as simulated annealing [113] and particle swarm optimisation [66] did not give better results.

Please note that results are only valid for the measured HVAC. Since the bypass is tuned for every unit manually, results cannot be transferred to other units. That insight led to the fact, that controller design in Chapter 5 was done using a new, linear HVAC model (see Section 5.4.1).

\subsubsection{Other parts}

In addition, the HVAC consists of other parts, like for instance the mixing chamber or fans. Estimating parameters for these parts was not forgotten; it was simply not necessary. As discussed in Chapter 3, parts were modelled analytically. Sometimes no further parameters are needed, such as for the mixing chamber and sometimes parameters could be measured directly, such as for the fan. Therefore, an estimation of parameters was not necessary.

\subsection{Controller model of the current controller}

Since the controller model was derived from the original source code, it was not necessary to estimate parameters for the model. Deviations may occur because the controller operates with a frequency of $10 \mathrm{~Hz}$, thus, is executed every $100 \mathrm{~ms}$ and measurement data are sampled with $10 \mathrm{~s}$ or that the actually implemented set point curve for the $T_{\text {IDA }}^{\text {set }}$ is not known.

\subsection{Thermal comfort model}

After the thermal comfort definition in Section 2.1.5 a question remains: how to express thermal comfort in numbers. Three different models are used:

- the original set point curve provided by the operator

- adapted set point curves, which can be gained from measurement data and 
- a linearisation of predicted mean vote (PMV)/percentage persons dissatisfied (PPD), so they can be used as weighting matrix in the objective function of the model predictive controller (MPC).

The following describes thermal comfort models for the adapted set point curve as well as the linearised PMV/PPD. Figure 4.3 shows the original set point curve and Equation (4.25) gives the matching equation.

\subsubsection{Fitted set point curves}

During the simulation of data from on-site measurements, notable deviations occurred between measured and simulated indoor air. Several explanations are possible: the maximum power of the HVAC may not be sufficient to keep the indoor air temperature constant. It is also possible, the control algorithm implemented in simulation does not match to the current controller that is running in the tram or the current set point curve is unknown.

Since the deviations between set point and current value occurred also at moderate indoor air temperatures and the controller did not trigger maximum HVAC performance, it could be excluded that performance limits were reached. The responsible technician described the implemented controller algorithm and after becoming aware of the differences the controller algorithms were checked once more. There exists a simple adjustment possibility for the set point curve. Therefore, it was assumed that the actually coded set point curve was unknown.

\section{Available data}

All available data of the period 2010-09-01 - 2011-08-31 were used. In total, about 250 files containing about 1.8 million measurements were analysed. That equals in turn about 5200 measurement hours. Before the data analysis could start, it had to be checked that measurement data are representative or unbiased. For that purpose, it was determined how many hours a given outdoor air temperature occurred during the year. This is plotted in Figure 4.4. An expected distribution for the outdoor air can be seen in Figure 4.4. Temperatures in the range of $10^{\circ} \mathrm{C}$ to $20^{\circ} \mathrm{C}$ occur more often than extremal values $\left(0^{\circ} \mathrm{C}\right.$ and $\left.35^{\circ} \mathrm{C}\right)$. There was no measurement in the range from $-20^{\circ} \mathrm{C}$ to $0^{\circ} \mathrm{C}$, this range was not considered further.

\section{Measurement results}

Figure 4.3 plots the indoor air temperature against the outdoor air temperature. The figure also shows the original set point curve. If there is good agreement between the original set point curve and the actual set point curve there would be as many values below as above the curve. This is roughly the case for the outdoor air range from $10^{\circ} \mathrm{C}$ to $20^{\circ} \mathrm{C}$. For the temperature ranges below and above, one can recognise notable deviations. 


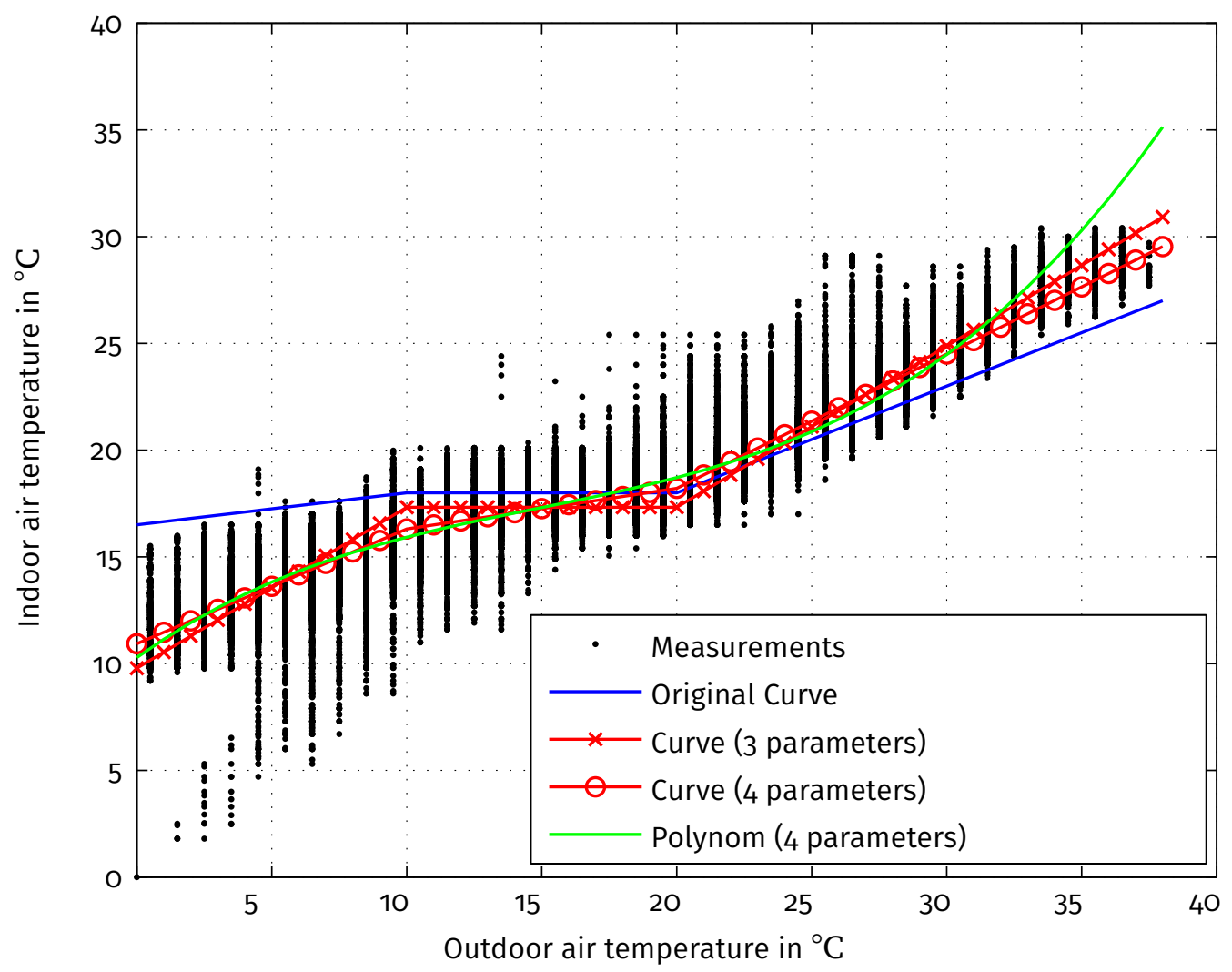

Figure 4.3. - Occurring indoor air temperature versus outdoor air temperature with original set-point curve and new calculated curve

\section{Set point curves}

According to the original set point curve, the indoor air temperature set point $T_{\text {IDA }}^{\text {set }}$ can be calculated by

$$
T_{\mathrm{IDA}}^{\mathrm{set}}=\left\{\begin{array}{lr}
8 & T_{\mathrm{ODA}}<-20 \\
0.7 \cdot T_{\mathrm{ODA}}+22 & -20 \leq T_{\mathrm{ODA}}<-10 \\
0.15 \cdot T_{\mathrm{ODA}}+16.5 & -10 \leq T_{\mathrm{ODA}}<10 \\
18 & -10 \leq T_{\mathrm{ODA}}<20 \\
0.5 \cdot T_{\mathrm{ODA}}+8 & T_{\mathrm{ODA}} \geq 20
\end{array} .\right.
$$

The set point curve is described by four parameters in the outdoor air temperature range from $0{ }^{\circ} \mathrm{C}$ to $35^{\circ} \mathrm{C}$ if the breakpoints of the function remain unchanged. If the $2^{\text {nd }}$ gradient is fixed at zero, one obtains a set point curve with 3 parameters.

Two different curves have been estimated by the least squares method, one with three parameters $\left(2^{\text {nd }}\right.$ gradient fixed $)$ and one with four parameters ( $2^{\text {nd }}$ gradient variable). Table 4.3 lists the gained parameters with the original values. In addition, a $3^{\text {rd }}$-degree polynomial curve was estimated. It 


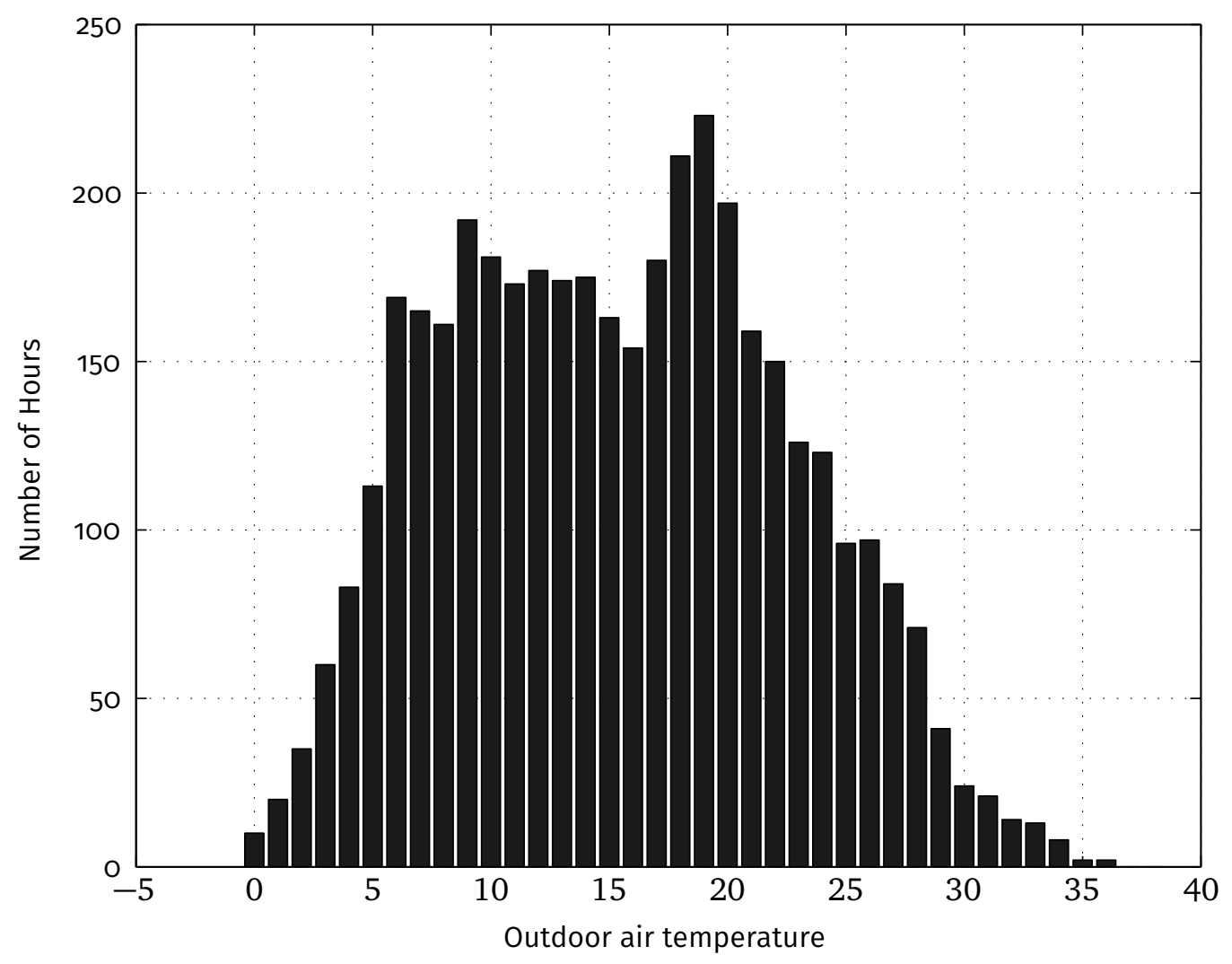

Figure 4.4. - Number of hours for a given outdoor air temperature

contains as many parameters as the piecewise linear set point curve with four parameters. Equation (1.2) shows the function in a generic form and Equation (1.3) lists the calculated numerical values. The function is

In addition, a $3^{\text {rd }}$ degree polynomial curve was estimated. It contains the same number of parameters as the piecewise linear set point curve (four parameters). The function is given in a generic form in Equation (4.26), calculated numerical values are listed in Equation (4.27). The function is

$$
T_{\mathrm{IDA}}^{\mathrm{set}}=f_{1} \cdot\left(T_{\mathrm{ODA}}\right)^{3}+f_{2} \cdot\left(T_{\mathrm{ODA}}\right)^{2}+f_{3} \cdot T_{\mathrm{ODA}}+f_{4},
$$

where

$$
f_{1}=0.00096954, \quad f_{2}=-0.043318, \quad f_{3}=0.90006, \quad f_{4}=10.2821,
$$

applies. 


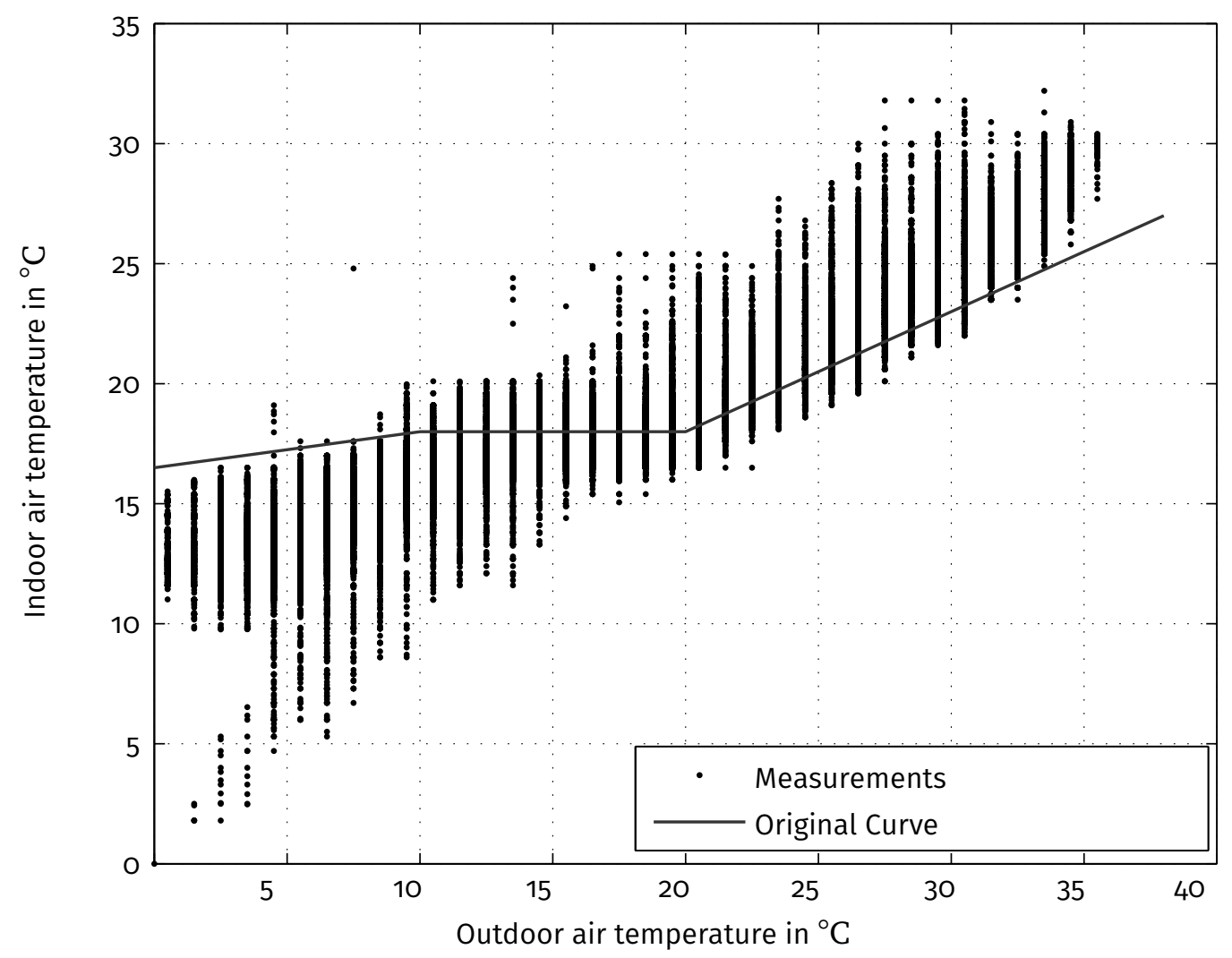

Figure 4.5. - Indoor air temperature versus outdoor air temperature, measurement points and set point curve

\subsubsection{Predicted mean vote}

The PMV or the derived PPD must be stated as a quadratic function of states to use them in the model predictive controller. [61] provides equations for calculating both values and it also shows an example implementation in Pascal. The implementation was transferred into Matlab. According to [61], PMV follows from the non-linear function

$$
\mathrm{PMV}=\mathrm{PMV}\left(\mathrm{MET}, \mathrm{WME}, \mathrm{CLO}, T_{A}, T_{R}, v_{a r}, \mathrm{RH}\right) .
$$

In which variables have the following meaning:

- MET - Metabolic rate in $\mathrm{W} / \mathrm{m}^{2}$

- WME - External work, normally around 0 in $\mathrm{W} / \mathrm{m}^{2}$

- CLO - Clothing, thermal isolation of clothing in $\mathrm{m}^{2} \mathrm{~K} / \mathrm{W}$

- $T_{A}$ - Air temperature in ${ }^{\circ} \mathrm{C}$

- $T_{R}$ - Radiation temperature in ${ }^{\circ} \mathrm{C}$

- $v_{a r}$ - Air velocity in $\mathrm{m} / \mathrm{s}$

- RH - Relative humidity in \% 
Table 4.3. - Parameter of various set point curves

\begin{tabular}{llll}
\hline & \multicolumn{3}{c}{ parameters } \\
\cline { 2 - 4 } Title & original & 3 estimated & 4 estimated \\
\hline intercept & 18 & 17.3 & 4.4 \\
$1^{\text {st }}$ gradient $\left(0{ }^{\circ} \mathrm{C}\right.$ to $\left.10{ }^{\circ} \mathrm{C}\right)$ & 0.15 & 0.75 & 0.54 \\
$2^{\text {nd }}$ gradient $\left(10^{\circ} \mathrm{C}\right.$ to $\left.20^{\circ} \mathrm{C}\right)$ & 0 & 0 & 0.19 \\
$3^{\text {rd }}$ gradient $\left(20^{\circ} \mathrm{C}\right.$ to $\left.35^{\circ} \mathrm{C}\right)$ & 0.5 & 0.76 & 0.63 \\
\hline
\end{tabular}

Table 4.4. - Parameters of the polynomial curve with 4 parameters

\begin{tabular}{ll}
\hline Description & Value \\
\hline$f_{1}$ & 0.00096954 \\
$f_{2}$ & -0.043318 \\
$f_{3}$ & 0.90006 \\
$f_{4}$ & 10.2821 \\
\hline
\end{tabular}

The air temperature $T_{A}$ matches the $T_{\text {IDA }}$. The radiation temperature is equal to the $T_{R}$. Thus, the modelling neglects the influence of direct solar radiation but without shading, no comfortable environment would be possible in direct sunlight. The absolute humidity $R H$ matches $Y_{\text {IDA }}$. Assumptions have to be made for the other variables. $v_{a r}$ is assumed to be $v_{a r}=0.1 \mathrm{~m} / \mathrm{s}$ because the airspeed was not measured during operation. A recommendation for MET can be found in [61], so, $M E T=1.2$ and $W M E=0$ was used. The clothing factor follows from worn clothing. For a summer day, $C L O=0.6$ was assumed. This value must be adapted to the season. The non-linear PMV and PPD function were calculated on grid points. Figure 4.6 shows a comparison between original and estimated function for the PPD. These points were used for a least squares estimation. The estimated function was of the following shape

$$
\begin{aligned}
\mathrm{PMV}^{e s t} & =x^{T} \cdot \mathbf{Q}_{\mathrm{PMV}} \cdot x+\mathbf{q}_{\mathrm{PMV}} \cdot x+\text { constant } \text { and } \\
\mathrm{PPD}^{\text {est }} & =x^{T} \cdot \mathbf{Q}_{\mathrm{PPD}} \cdot x+\mathbf{q}_{\mathrm{PPD}} \cdot x+\text { constant }
\end{aligned}
$$


where

$$
\begin{aligned}
\mathbf{Q}_{\mathrm{PMV}} & =\left[\begin{array}{cccc}
257.3186 & 0 & 0 & 0 \\
243.1137 & 243.8957 & 0 & 0 \\
119.8109 & 114.8446 & 60.4857 & 0 \\
0 & 0 & 0 & 0
\end{array}\right] \\
\mathbf{q}_{\mathrm{PMV}}= & {\left[\begin{array}{cccc}
6.3709 & 6.0507 & 2.5367 & 0
\end{array}\right] } \\
\mathbf{Q}_{\mathrm{PPD}}= & {\left[\begin{array}{cccc}
0.3267 & 0 & 0 & 0 \\
0.59594 & 0.26733 & 0 & 0 \\
0.16419 & 0.1458 & 0.020203 & 0 \\
0 & 0 & 0 & 0
\end{array}\right] } \\
\mathbf{q}_{\mathrm{PPD}}= & {\left[\begin{array}{cccc}
-32.2887 & -28.9624 & -7.9987 & 0
\end{array}\right] }
\end{aligned}
$$

applies. Since $\mathbf{Q}_{\mathrm{PPD}}$ is not positiv semidefinite, PPD must not be used as weighting matrix in the MPC. 


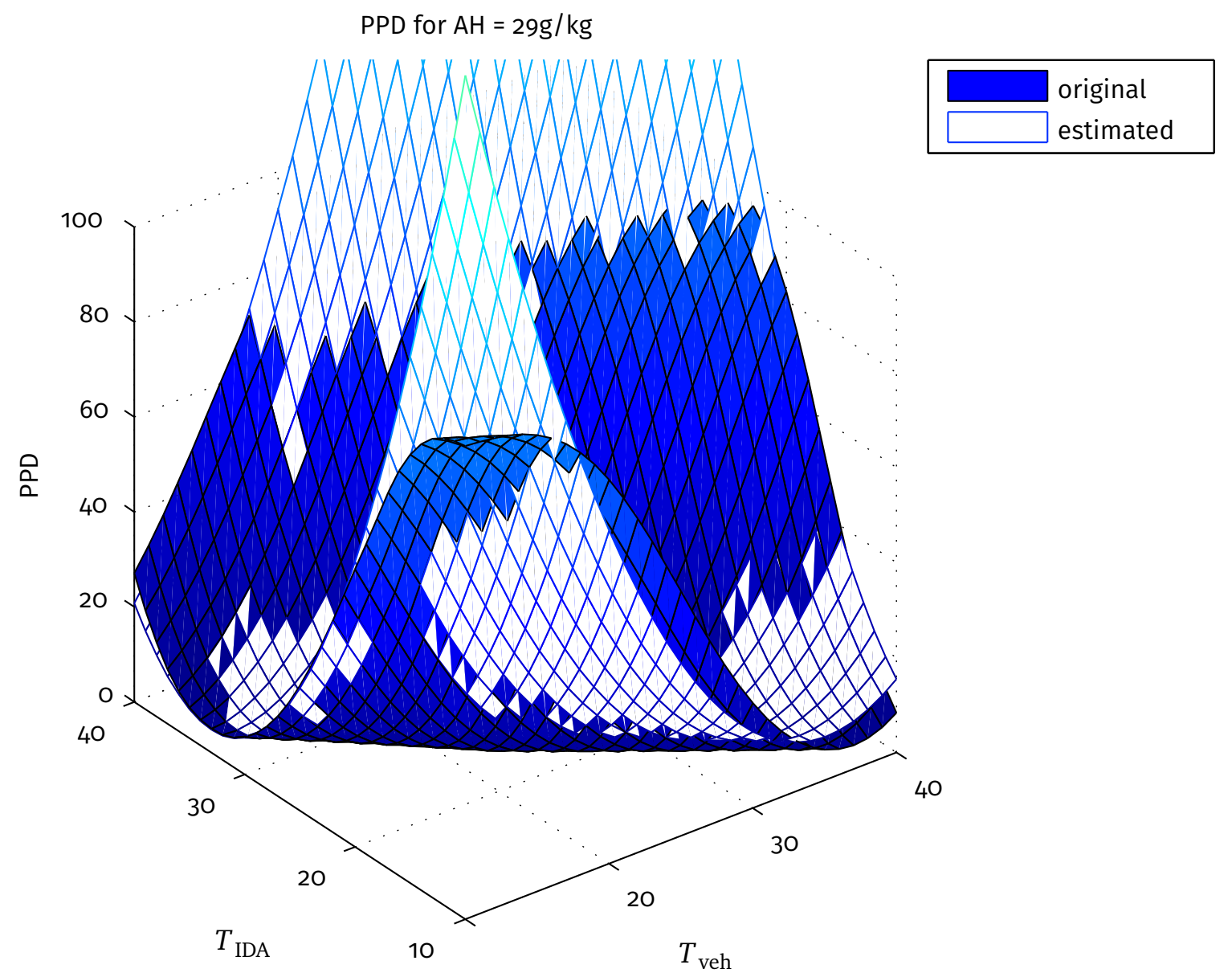

Figure 4.6. - Comparing estimated with original PPD for a $T_{\text {IDA }}$ and $T_{\text {veh }}$ temperature range with fixed humidity. 


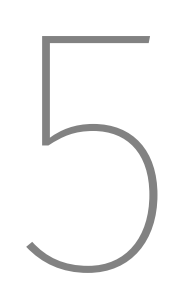

\section{Controller Design}

This chapter describes the design of the new controller, which is based on the developed models of the tram and the HVAC. The introduction presents the thermal comfort control problem and discusses various problem formulations. It uses one of these formulations to state the new control loop. The following sections design the controllers and describe their implementation on a RCPP.

\subsection{Introduction}

This section defines the requirements for the controller design and discusses the current control loop and its implementation. Then it lists various improvement measures for the HVAC in case they affect the controller. In addition, the sections explain the improvement measures of the controller and discuss the thermal comfort control problem, define the control objective and describe its mathematical formulation.

Section 5.2 creates a new control loop, based on the most suitable mathematical formulation.

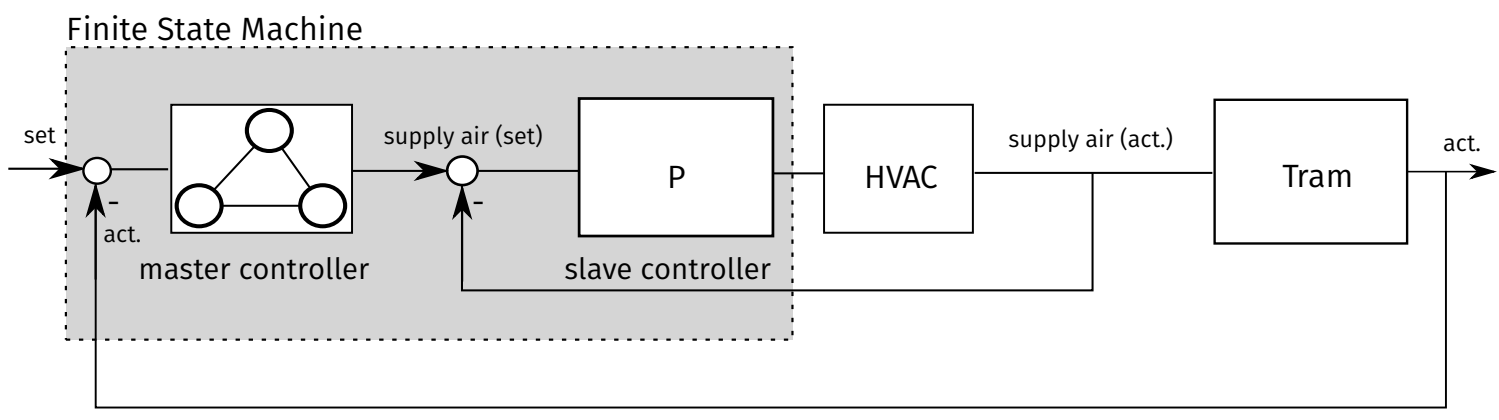

Figure 5.1. - Current control loop. The current control loop depends on the operational mode. It shows the control while in Heating mode with an operating electric heater.

\subsubsection{Current control loop}

Section 3.1.2 has already introduced the closed control loop and Section 3.2, Section 3.3 and Section 3.4 have discussed its individual parts. Figure 5.1 exemplary shows the currently used 
implementation as a block diagram. A switch (not shown) allows to choose the operational mode (Preheat, Auto, Off). When Auto is chosen, the FSM switches to heating mode and the electric heater is active, then a P-controller is realised through the transaction conditions of the FSM. In all other cases, the slave control loop does not apply. From the control engineering point of view, the master controller is a combination of several multi-point controllers.

\subsubsection{Improvement measures}

The new controller enables setting up the following measures planned for the HVAC. The document [112] describes the improvement measures and the following list summarises them:

- Variable frequency compressor The compressor of the CRM needs to be usable over a wide working range. Currently the compressor is On or Off only.

- Load-dependent fresh airflow The supply air fan must have as many control levels as possible. Currently, it has only three levels. It supplies an outdoor airflow into the tram to keep the carbon dioxide concentration low. The outdoor airflow should be as low as possible, but the carbon dioxide concentration must not exceed an upper limit.

- Heat pump Instead of introducing heat into the tram by an electric heater, an extra heat pump must be installed.

- Electric heater Instead of pulsing the electric heater in three levels, the new electric heater should be switchable in percentage levels.

The following measures are planned for the controller:

- Set point curve - passenger comfort The set point curve for the tram should be adapted or an alternative method should be used to rate the passenger comfort.

- Free cooling The controller should cool the tram as long as possible by increasing the supply airflow.

- Control of the fresh airflow depending on the passenger load

- Control performance

The new controller has to fulfil all the above-mentioned measures in a robust way.

\subsubsection{Control objectives}

The control problem of thermal comfort is: In the tram, a high thermal comfort has to be achieved for the passenger, whereas simultaneously the electrical power consumption of the HVAC needs to be as small as possible. Both objectives contradict each other. This and the next subsection describe how the conflict can be solved.

Figure 5.2 graphs both contradicting objectives. The objective is to find a solution in which the electrical energy consumption is as small as possible but creates a thermal comfort as high as possible. The abscissa shows the thermal comfort and the ordinate the electrical energy consumption. The grey area depicts the solution set. The target solution of this set is as near as possible to the 


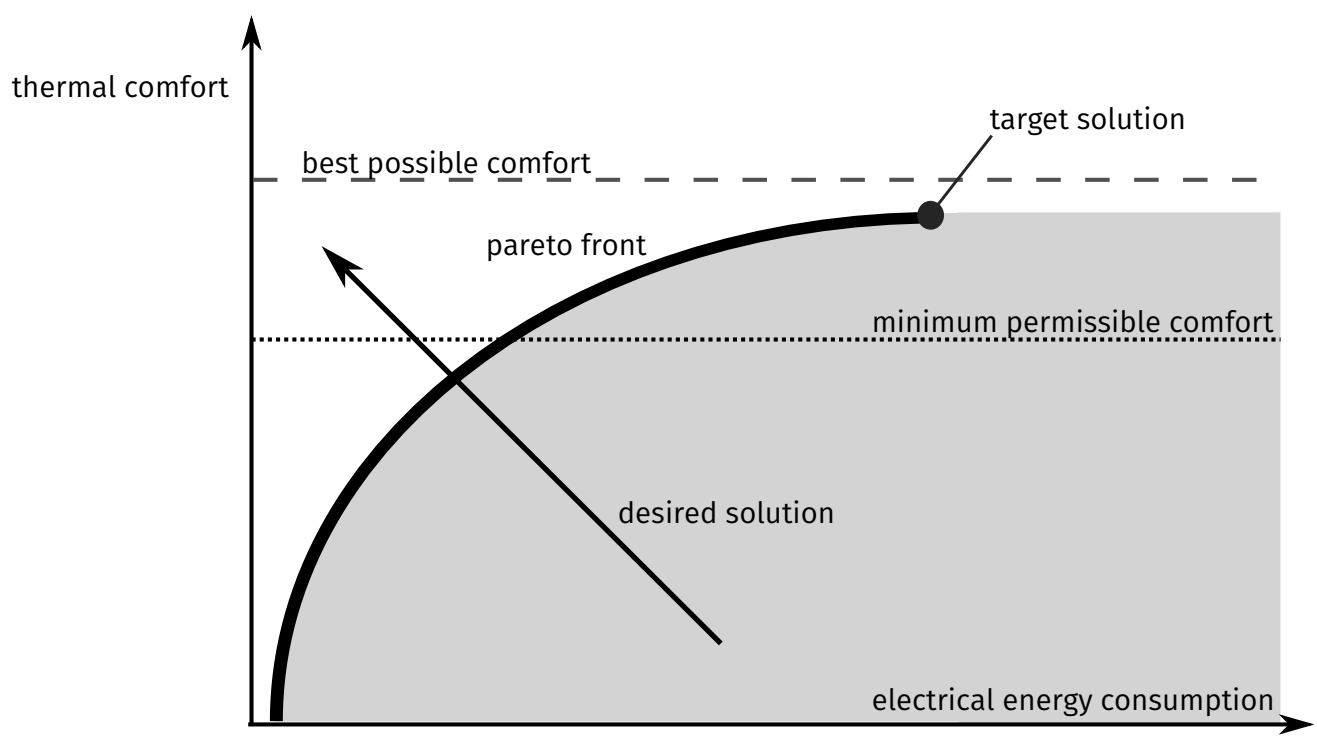

Figure 5.2. - Pareto optimality of the thermal comfort control problem

upper left corner. A black arrow points in this direction. The best possible comfort is constant (grey dashed) and cannot be fully achieved. The operator of the tram defines a minimum feasible comfort (grey dotted line). All Pareto-optimal solutions are on the upper left boundary of the solution set (black curve, Pareto front). The target solution (shown as a grey dot) is as near as possible to the best possible comfort and has a low electrical energy consumption (i.e. is an element of the Pareto front).

This chapter describes how these solutions can be found. A multi-criteria optimisation problem is an optimisation problem with two or more contradicting objectives.

\subsubsection{Multi-criteria optimisation problem}

The problem described above agrees exactly to a multi-criteria optimisation problem as Chapter 2 cites it. Kallrath [65, p. 160f] wrote that this problem can be solved with goal programming and distinguishes two variants:

1. the pre-emptive variant and

2. the Archimedean variant [65, p. 160].

The following paragraphs discuss applying those two variants to the thermal comfort problem.

\section{Pre-emptive variant}

The pre-emptive variant ranks the goals differently. First, the priorities of the goals must be specified for the tram.

When the controller sets the priority to the electrical energy consumption, it is easy to see that the optimal solution is identical with the trivial solution. The HVAC consumes the smallest amount 
of energy, it is switched off. This solution is usually not satisfying because the achieved thermal comfort is unacceptable. One possibility to calculate this variant would be to consider the thermal comfort as a constraint in the optimisation. This means that the electrical energy consumption is minimised subject to the thermal comfort. Then this would not be a multi-criteria optimisation problem any more.

The second possibility is to set a priority to the thermal comfort and afterwards to the electrical energy consumption. This chapter follows this approach further.

\section{Archimedean variant}

The Archimedean variant combines both goals into a new joint goal. For the tram, the monetary cost minimisation offers itself, expressed in the unit Euro. Acceptable factors must be found for converting both goals into the joint goal. For the electrical power consumption, this is given by the electricity rate. For converting the thermal comfort into Euro, transport services need to specify the factor. No rule of thumb exists for this conversion factor, which is a critical disadvantage of this variant.

\subsection{New control loop}

Based on the idea of the pre-emptive variant, the problem is split into two subproblems. First, auxiliary variables are calculated by solving the optimisation problem for the optimal thermal comfort. Then, the before calculated auxiliary variable provides a set point and searches an energyoptimal switching variation for the HVAC. The new controller is a cascaded controller like the current control loop. The master control loop sets the optimal thermal comfort and the slave control loop transforms this set point in an energy-optimal way into switching commands for the HVAC.

\subsection{Master controller}

This section describes the design of the master controller as a model predictive controller. For this design, the tram model stated in Section 3.2 needs to be linearised and transformed into a suitable representation (for example to a state-space representation). Afterwards, one can start with the controller design by expressing the underlying quadratic optimisation problem, which can be solved by a proper solution method. The following subsections describe linearising the tram model, the approach of the optimisation problem and the solution method.

\subsubsection{Tram model}

Chapter 3 describes the analytical thermal model of the tram in detail. This subsection summarises, linearises and transforms the model of the tram into a state-space representation. 


\section{Summarising the tram model}

The tram model needs to calculate the following state variables $x$ of the tram:

- indoor air temperature $T_{\text {IDA }}$

- temperature of the car body $T_{\text {veh }}$

- absolute humidity of indoor air $Y_{\text {IDA }}$

- carbon dioxide concentration of indoor air $\sigma_{\text {IDA }}$.

Non-linear differential equations result from the balance equations. The various variables can be calculated by inserting the proper terms into the differential equations. According to Equation (3.1), for the indoor air temperature follows

$$
\begin{aligned}
C_{\mathrm{IDA}} \cdot \dot{T}_{\mathrm{IDA}} & =k \cdot O \cdot\left(T_{\mathrm{ODA}}-T_{\mathrm{IDA}}\right)-k_{\mathrm{veh}} \cdot\left(T_{\mathrm{IDA}}-T_{\mathrm{veh}}\right) \ldots \\
& +\dot{m}_{\mathrm{SUP}} \cdot c_{\mathrm{air}} \cdot\left(T_{\mathrm{SUP}}-T_{\mathrm{IDA}}\right)+k_{\mathrm{pas}, \mathrm{CO} 2} \cdot n_{\mathrm{pas}}+k_{\mathrm{sol}} \cdot \dot{q}_{\mathrm{sol}} \cdot
\end{aligned}
$$

For the car temperature

$$
C_{\mathrm{veh}} \cdot \dot{T}_{\mathrm{veh}}=k_{\mathrm{veh}} \cdot\left(T_{\mathrm{IDA}}-T_{\mathrm{veh}}\right)
$$

applies. Equation (5.3) allows calculating the absolute humidity of the indoor air

$$
V_{\mathrm{IDA}} \cdot \rho_{\mathrm{IDA}} \cdot \dot{Y}_{\mathrm{IDA}}=\dot{m}_{\mathrm{SUP}} \cdot\left(Y_{\mathrm{SUP}}-Y_{\mathrm{IDA}}\right)+k_{\mathrm{pas}, \mathrm{lat}} \cdot n_{\mathrm{pas}} \cdot
$$

Equation (5.4) allows calculating the carbon dioxide concentration

$$
V_{\mathrm{IDA}} \cdot \rho_{\mathrm{IDA}} \cdot \dot{\sigma}_{\mathrm{IDA}}=\dot{m}_{\mathrm{SUP}} \cdot\left(\sigma_{\mathrm{SUP}}-\sigma_{\mathrm{IDA}}\right)+k_{\mathrm{pas}, \mathrm{CO} 2} \cdot n_{\mathrm{pas}} \cdot
$$

State, input and output variables As in previous sections, the state variables $x$ is

$$
x=\left[\begin{array}{llll}
T_{\text {IDA }} & T_{\text {veh }} & Y_{\text {IDA }} & \sigma_{\text {IDA }}
\end{array}\right]^{T} .
$$

The control variable $u$ is the part of the inputs manipulated by the controller. As known from Chapter 3, the HVAC changes the state of the supply air. The state of the supply air includes the temperature, the relative humidity and the carbon dioxide concentration. As the HVAC produces the mass flow of the supply air, it can also be changed.

When looking at Equation (5.1), one realises that the product is formed of both control variables. Thus, the expression $\dot{m}_{\mathrm{SUP}} \cdot c_{\text {air }} \cdot\left(T_{\mathrm{SUP}}-T_{\mathrm{IDA}}\right)$ is non-linear in the control variables. This fact is also true for the absolute humidity $\dot{m}_{\text {SUP }} \cdot\left(Y_{\text {SUP }}-Y_{\text {IDA }}\right)$ and the carbon dioxide concentration $\dot{m}_{\text {SUP }} \cdot\left(\sigma_{\text {SUP }}-\sigma_{\text {IDA }}\right)$.

Substitution To transform Equation (5.1), Equation (5.3) and Equation (5.4) into a linear representation, the supply air terms are substituted. The following relationships apply to the substitution

$$
\begin{aligned}
& \Delta h_{\mathrm{SUP}}=\dot{m}_{\mathrm{SUP}} \cdot c_{\mathrm{air}} \cdot\left(T_{\mathrm{SUP}}-T_{\mathrm{IDA}}\right), \\
& \Delta Y_{\mathrm{SUP}}=\dot{m}_{\mathrm{SUP}} \cdot\left(Y_{\mathrm{SUP}}-Y_{\mathrm{IDA}}\right) \text { and } \\
& \Delta \sigma_{\mathrm{SUP}}=\dot{m}_{\mathrm{SUP}} \cdot\left(\sigma_{\mathrm{SUP}}-\sigma_{\mathrm{IDA}}\right) .
\end{aligned}
$$


Thus, the linear control variables result in

$$
u=\left[\begin{array}{lll}
\Delta h_{\mathrm{SUP}} & \Delta Y_{\mathrm{SUP}} & \Delta \sigma_{\mathrm{SUP}}
\end{array}\right]^{T} .
$$

The disturbance $z$ is

$$
z=\left[\begin{array}{lll}
T_{\mathrm{ODA}} & n_{\mathrm{pas}} & \dot{q}_{\mathrm{sol}}
\end{array}\right]^{T} .
$$

The current values $y$ are equal to the states according to Equation (5.5) so that the state space system can be developed.

\section{State space representation}

The state space representation of a continuous system is, as known from Chapter 2,

$$
\begin{aligned}
\mathbf{F}_{\mathbf{c}} \dot{x} & =\mathbf{A}_{\mathbf{c}} x+\mathbf{B}_{\mathbf{c}} u+\mathbf{E}_{\mathbf{c}} z \\
y & =\mathbf{C}_{\mathbf{c}} x+\mathbf{D}_{\mathbf{c}} u .
\end{aligned}
$$

According to Equation (5.1) to Equation (5.4)

$$
\begin{aligned}
& \mathbf{A}_{\mathbf{c}}=\left[\begin{array}{rrrr}
-k-k_{\mathrm{veh}} & k_{\mathrm{veh}} & 0 & 0 \\
k_{\mathrm{veh}} & -k_{\mathrm{veh}} & 0 & 0 \\
0 & 0 & 0 & 0 \\
0 & 0 & 0 & 0
\end{array}\right] \\
& \mathbf{B}_{\mathbf{c}}=\left[\begin{array}{lll}
1 & 0 & 0 \\
0 & 0 & 0 \\
0 & 1 & 0 \\
0 & 0 & 1
\end{array}\right] \\
& \mathbf{E}_{\mathbf{c}}=\left[\begin{array}{rrr}
k & k_{\mathrm{pas}, \text { sen }} & k_{\mathrm{sol}} \\
0 & 0 & 0 \\
0 & k_{\mathrm{pas}, \mathrm{lat}} & 0 \\
0 & k_{\mathrm{pas}, \mathrm{CO} 2} & 0
\end{array}\right] \\
& \mathbf{F}_{\mathbf{c}}=\operatorname{diag}\left(\left[\begin{array}{llll}
C_{\mathrm{IDA}} & C_{\mathrm{veh}} & m_{\mathrm{IDA}} & m_{\mathrm{IDA}}
\end{array}\right]\right)
\end{aligned}
$$

applies to the matrices of the system.

\subsubsection{Optimisation problem}

For each of the three state variables $T_{\text {IDA }}, Y_{\text {IDA }}$ and $\sigma_{\text {IDA }}$ a set point $w$ exists which should be met by the controller as precisely as possible. The scope of work leads to the solution approach, which is based on the least squares method by minimising the sum of the squared control error $\boldsymbol{e}$ over the control horizon $T$. For this purpose, the sequence of the control variables $u$ is calculated for the horizon $T$. In addition, constraints for the absolute control variable $u$, the control variable change $\Delta u$ as well as for the state variable $x$ have to be considered. Section 2.3.3 contains a more detailed description of the MPC problem. 
The constraints in $x$ and $u$ can be easily considered by adding them to the optimisation problem. This is more challenging for $\boldsymbol{e}$ and $\Delta u$ because they are not present yet in the current formulation of the optimisation problem. The following paragraph extends the state vector and the state space system with these variables.

Augmentation

The state vector $x \in \mathbb{R}^{n}$ is extended with the control error vector $e \in \mathbb{R}^{p}$ and the vector of the absolute controller output $u \in \mathbb{R}^{m}$. Thus, one gets a new state vector and the new control variable

$$
\begin{aligned}
\tilde{x}=[x, \boldsymbol{e}, u]^{T} & \in \mathbb{R}^{\tilde{n}}, \\
\tilde{u}=\Delta u & \in \mathbb{R}^{m} .
\end{aligned}
$$

The absolute control variable at time $t$ can be calculated from the sum of the absolute control variable at time $t-1$ and the change $\Delta u$

$$
u(t)=u(t-1)+\Delta u(t)
$$

The control error at time $t$ is

$$
\boldsymbol{e}(t)=w(t)-x(t)
$$

So the new matrices $\widetilde{\mathbf{A}}$ and $\widetilde{\mathbf{B}}$ follow

$$
\widetilde{\mathbf{A}}=\left[\begin{array}{rrr}
\mathbf{A}_{\mathbf{d}} & \mathbf{0}^{n \times p} & \mathbf{B}_{\mathbf{d}} \\
\mathbf{A}_{\mathbf{d}}^{\text {ref }} & \mathbf{0}^{p \times p} & \mathbf{B}_{\mathbf{d}}^{\text {ref }} \\
\mathbf{0}^{m \times n} & \mathbf{0}^{m \times p} & \mathbb{1}^{m \times m}
\end{array}\right] \in \mathbb{R}^{\tilde{n} \times \tilde{n}} \quad \widetilde{\mathbf{B}}=\left[\begin{array}{r}
\mathbf{B}_{\mathbf{d}} \\
\mathbf{B}_{\mathbf{d}}^{\text {ref }} \\
\mathbb{1}^{m \times m}
\end{array}\right] \in \mathbb{R}^{\tilde{n} \times m}
$$

Thereby $\mathbf{A}_{\mathbf{d}}$ is the discretised product from $\mathbf{F}_{\mathbf{c}}{ }^{-1} \mathbf{A}_{\mathbf{c}}$, in which the product was discretised using a zero order hold and a sampling time of $10 \mathrm{~s}$. This was done analogously for $\mathbf{B}_{\mathbf{d}}$. In $\mathbf{A}_{\mathbf{d}}^{\text {ref }}$ and $\mathbf{B}_{\mathbf{d}}^{\text {ref }}$ the rows from $\mathbf{A}_{\mathbf{d}}$ respectively $\mathbf{B}_{\mathbf{d}}$ were taken, which matter for calculating the corresponding errors. The first row of $\mathbf{A}_{\mathbf{d}}$ matrix was used for calculating the control error of the first state $T_{\text {IDA }}^{\text {ref }}-T_{\text {IDA }}$.

An extra simplification was made for the disturbance matrix $\mathbf{E}_{\mathbf{d}}$. The disturbance $z$ must be known for the entire horizon $T$ to be considered. It therefore suffices to use the product $E z=\mathbf{E}_{\mathrm{d}} z$, in which $E z \in \mathbb{R}^{n}$ applies to further calculations. For the disturbance, the same augmentation as for $\widetilde{\mathbf{A}}$ and $\widetilde{\mathbf{B}}$ has to be done. One gets

$$
Z=\left[\begin{array}{r}
E z \\
E z^{\mathrm{ref}}-w \\
0^{m}
\end{array}\right] \quad \in \mathbb{R}^{\tilde{n}}
$$


Discrete, augmented state space system

With the augmented matrices, the discrete state space system can be formulated

$$
\begin{aligned}
x(t+1) & =\mathbf{A}(t) x(t)+\mathbf{B} u(t)+Z(t) \\
y & =x(t)
\end{aligned}
$$

$Z$ is a function of time. Only in this way, for example, set point steps can be correctly considered. In addition, the disturbance usually changes over the horizon because it is not constant. However, this circumstance is not obvious for A. The explanation of Equation (3.7) discusses the $k$ value. It is a function of the tram speed $v$. As trains operate on a defined schedule, $v(t)$ is known and therefore $k(t)$ applies. This is called a linear time-variant state space system. This relationship has to be considered during selecting the solution procedure and is an important requirement for the same. To express the constraints, an anticipation to Section 5.4.2 is necessary. The HVAC is described in a way causing the constraints for $x(t)$ are (non-linear) dependent on the current state and may change dramatically in every time step. The constraints must therefore be considered as time-varying. The weighting matrices are usually constant on the horizon, but one expects a better behaviour of time-varying weighting matrices in certain states. Thus, $\mathbf{R}$ and $\mathbf{Q}$ should also be implemented as time-varying.

\section{Problem formulation}

Equation (5.23) gives the discrete augmented state space system. Possibly occurring disturbances are random variables. The cost function of the MPC is, as a function of time $t$

$$
J(t)=\frac{1}{T} \sum_{t=0}^{T-1}\left(\widetilde{x}(t)^{T} \mathbf{Q}(t) \widetilde{x}(t)+\widetilde{u}(t)^{T} \mathbf{R}(t) \widetilde{u}(t)+q(t) \widetilde{x}(t)+r(t) \widetilde{u}(t)\right) .
$$

Both matrices $\mathbf{Q} \in \mathbb{R}^{\tilde{n} \times \tilde{n}}$ and $\mathbf{R} \in \mathbb{R}^{m \times m}$ must be positive semi-definite. In addition $\mathbf{q} \in \mathbb{R}^{\tilde{n}}$ and $\mathbf{R} \in \mathbb{R}^{m}$ apply. The mixed terms $\widetilde{x}(t)^{T} \mathbf{S} \widetilde{x}(t)$ and $\widetilde{u}(t)^{T} \mathbf{S}^{T} \widetilde{x}(t)$ are neglected because $\mathbf{S}=0$ is assumed. The constraints in $\widetilde{x}(t)$ and $\widetilde{u}(t)$

$$
\begin{aligned}
\tilde{x}^{\mathrm{lb}}(t) \leq \widetilde{x}(t) \leq \widetilde{x}^{\mathrm{ub}}(t), & t=1, \ldots, T \\
\widetilde{u}^{\mathrm{lb}}(t) \leq \widetilde{u}(t) \leq \widetilde{u}^{\mathrm{ub}}(t), & t=0,1, \ldots, T
\end{aligned}
$$

can be written as $l$ linear inequalities

$$
\mathbf{F}_{1} \tilde{x}(t)+\mathbf{F}_{2} \widetilde{u}(t) \leq f(t), \quad t=0,1, \ldots, T .
$$

In the inequality $\mathbf{F}_{1} \in \mathbb{R}^{l \times \tilde{n}}, \mathbf{F}_{2} \in \mathbb{R}^{l \times m}$ and $f \in \mathbb{R}^{l}$ are known. The MPC problem is solved by solving the underlying quadratic problem (QP)

$$
\begin{array}{cl}
\text { minimise } & J(t), \quad t=0, \ldots, T-1 \\
\text { subject to } & \widetilde{x}(t+1)=\widetilde{\mathbf{A}}(t) \tilde{x}(t)+\widetilde{\mathbf{B}} \widetilde{u}(t)+Z(t) \\
& \mathbf{F}_{1} \widetilde{x}(t)+\mathbf{F}_{2} \widetilde{u}(t) \leq f
\end{array}
$$


in the variables

$$
\begin{aligned}
& x(1), \ldots, x(T) \\
& u(0), \ldots, u(T-1)
\end{aligned}
$$

and parameters

$$
x(0), \mathbf{A}(t), \mathbf{B}, Z(t), \mathbf{Q}(t), \mathbf{R}(t), q(t), r(t), \mathbf{F}_{1}(t), \mathbf{F}_{2}(t), f(t),
$$

in every time instance [119]. A partition of the horizon into control horizon $T_{c}$ and prediction horizon $T_{p}$ is omitted.

\subsubsection{Solution procedure}

First, the requirements for the solution procedure are summarised following from the scope of work. With this specification, a proper method was searched in the literature. The most suitable method was chosen and fitted to the requirements.

\section{Requirements}

The solution procedure must solve the MPC problem (see Section 2.3.3) for a linear time-varying state space system (compare Equation (5.23)) with time-varying constraints and time-varying weighting matrices in the cost function on a RCPP in about 10 times real-time. The requirement for 10 times real-time results because other algorithms must also be executed in this time also. For the selected sampling time of $10 \mathrm{~s}$, this results in a needed execution time of about $1 \mathrm{~s}$.

\section{Literature}

Due to the fact that the MPC needs to run on an embedded system, some Matlab functions are not available. This includes, In particular, the function quadprog [107], which is used to solve the quadratic problem and is the core of many implementations found on Mathworks Exchange.

Most alternative MPC Frameworks like YALMIP [80] use an external solver. Gurobi [43], CVXopt [21] and many more are used as solvers. Porting an existing solver to an embedded platform would require too much effort. Developing an own solver was also excluded for the same reason. The solution proposed by Boyd and Wang is written in C and well documented. It can be adapted with moderate effort for the embedded system. The paper of Boyd and Wang [119] shows the calculation process in detail.

\subsection{Slave controller}

Before one can continue with the design of the slave controller, the initial situation and scope are discussed. 


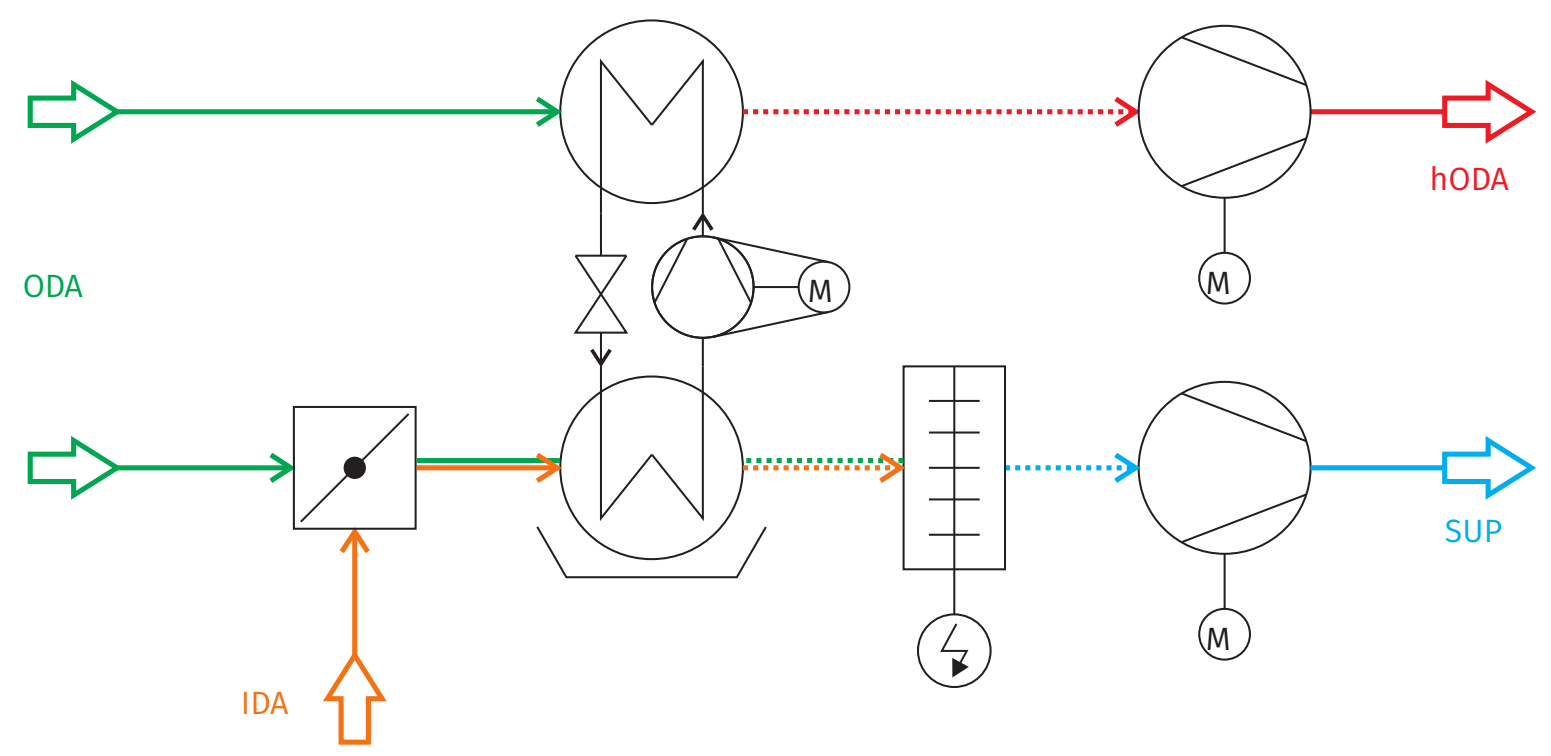

Figure 5.3. - Sketch of the new air conditioning

Initial situation The master controller provides set points for the slave controller. The slave controller meets these set points precisely by switching the parts of the air conditioning system.

Scope The scope of the slave controller includes, minimising the power consumption of the air conditioning system while satisfying the constraints. The following subsections present the basic analytical and thermal equations for the air conditioning system (see Section 5.4.1). The shown model in Chapter 3 is non-linear and, also because of the execution time it is not suitable for optimisation.

Linear equations are used for developing the mixed integer linear problem (MILP), in which proper variables have to be chosen. In addition, the problem formulation needs to consider various constraints (see Section 5.4.2). Section 5.4.3 reformulates the problem by a smarter variable choice so that the solution procedure can calculate solutions faster. With the chosen variables from the previous subsection, the actually applied manipulated variables can be calculated (see Section 5.4.4). Section 5.4.5 shows various solution methods for the presented optimisation problem.

\subsubsection{A linear HVAC model}

This subsection describes the air conditioning system and its parts by their physical relationships. It makes simplifications wherever possible to improve the efficiency of the calculation and to keep the accuracy as high as possible. For modelling the air conditioning system it is necessary to study the parts separately.

Parts It is obvious from the air conditioning design (see Figure 5.3) that it consists of the following parts (see Figure 5.4) 


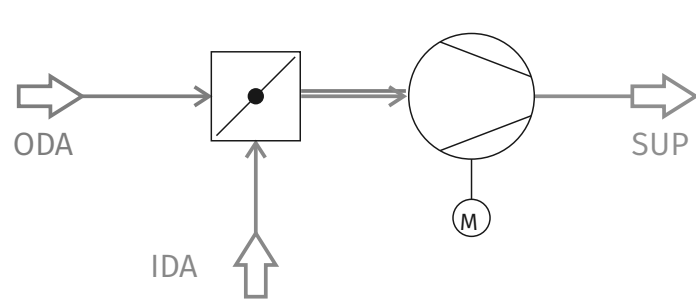

(a) Modelling of fan and mixing chamber

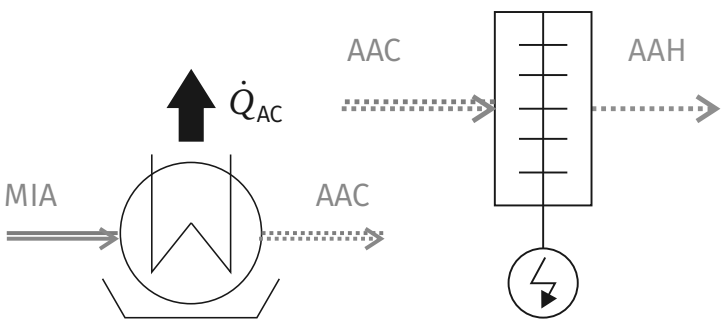

(b) Modelling of the (c) Schematic sketch of CRM the electric heater

Figure 5.4. - Schematic diagrams of different parts of the air conditioning system

- mixing chamber and supply air fan

- CRM

- heat pump and electric heater

The following section describes the individual parts of the air conditioning system in detail. It develops the overall mass balance, a partial mass balance of water, a partial mass balance carbon dioxide, and the energy balance for each part.

\section{Mixing chamber and supply air fan}

The mixing chamber and the supply air fan are summarised as a combined unit. Figure 5.4a shows a schematic diagram of the different parts. With those two parts, it is possible to control the demanded supply air mass flow and the needed outdoor air mass flow.

Mass balance The fundamental low of mass balances is the law of mass conservation, so that ingoing mass flows match outgoing mass flows. Thus, the mass balance for the mixing chamber and the fan is

$$
\dot{m}_{\mathrm{SUP}}=\dot{m}_{\mathrm{IDA}}+\dot{m}_{\mathrm{ODA}}
$$

This balance neglects condensing water at the evaporator of the CRM.

Energy balance The $1^{\text {st }}$ fundamental law of thermodynamics (see Section 2.1.1) states the energy conservation, which means that the ingoing energy flows must be equal to the outgoing energy flow

$$
\dot{m}_{\mathrm{SUP}} \cdot h_{\mathrm{MIA}}=\dot{m}_{\mathrm{IDA}} \cdot h_{\mathrm{IDA}}+\dot{m}_{\mathrm{ODA}} \cdot h_{\mathrm{ODA}} \text {, }
$$

The energy introduced by the fan does not change the temperature of the supply air. It is therefore neglected. 
Partial mass balance of water The mass preservation must also be valid for the water ratio of air. The partial mass balance of water follows from this

$$
\dot{m}_{\mathrm{SUP}} \cdot Y_{\mathrm{MIA}}=\dot{m}_{\mathrm{IDA}} \cdot Y_{\mathrm{IDA}}+\dot{m}_{\mathrm{ODA}} \cdot Y_{\mathrm{ODA}} \cdot
$$

Partial mass balance of carbon dioxide The partial mass balance of carbon dioxide is analogue to the partial mass balance of water. So it follows

$$
\dot{m}_{\mathrm{SUP}} \cdot \sigma_{\mathrm{SUP}}=\dot{m}_{\mathrm{IDA}} \cdot \sigma_{\mathrm{IDA}}+\dot{m}_{\mathrm{ODA}} \cdot \sigma_{\mathrm{ODA}} .
$$

Substitution It is usually an advantage to work with the individual mass flows $\dot{m}_{\mathrm{ODA}}$ and $\dot{m}_{\mathrm{IDA}}$ for the modelling. However, only the supply air mass flow $\dot{m}_{\text {SUP }}$ and the outdoor air ratio $\alpha$ can be controlled directly. The rotational speed of the fan controls $\dot{m}_{\text {SUP }}$ and the flap position of the mixing chamber controls $\alpha$. The outdoor air ratio of the supply air is

$$
\alpha=\frac{\dot{m}_{\mathrm{ODA}}}{\dot{m}_{\mathrm{SUP}}} .
$$

From the mass balance of the fan and the mixing chamber (see Equation (5.33)) the recirculation air ratio follows as

$$
\frac{\dot{m}_{\mathrm{IDA}}}{\dot{m}_{\mathrm{SUP}}}=1-\alpha
$$

Electrical power consumption The modelling neglects the power consumption for the flap control of the mixing chamber. According to the laws of proportionality [94, p. 1117],

$$
\dot{V}_{2}=\dot{V}_{1}\left(\frac{n_{2}}{n_{1}}\right)
$$

applies to the volume flow, and for the power consumption of the fan

$$
P_{2}=P_{1}\left(\frac{n_{2}}{n_{1}}\right)^{3}
$$

follows. The mass flow transported by the fan is directly proportional to the volume flow. The power consumption is proportional to the power of 3 of the mass flow. This law must be linearised for linear optimisation. A linear upper bound of the form

$$
P_{\text {fan }}=a_{\text {fan }} \cdot \dot{m}_{\mathrm{SUP}}-b_{\text {fan }}
$$

was assumed.

\section{Compression refrigeration machine}

The CRM in the built-in composition allows both heating and cooling. This subsection describes the cooling mode only. The subsection of the heat pump and electric heater (see below) describes the heating mode. Figure 5.4b shows a schematic sketch of the CRM. 
Mass balance of waters In the cooling mode, the condensing water on the cold heat exchanger surface must be considered for the water balance of the CRM. So, the mass balance of water considers it

$$
\dot{m}_{\mathrm{SUP}} \cdot Y_{\mathrm{MIA}}=\dot{m}_{\mathrm{ac}}+\dot{m}_{\mathrm{SUP}} \cdot Y_{\mathrm{AAC}}
$$

and reformulated to the mass of condensed water gives

$$
\dot{m}_{\mathrm{ac}}=\dot{m}_{\mathrm{SUP}} \cdot\left(Y_{\mathrm{MIA}}-Y_{\mathrm{AAC}}\right) .
$$

Energy balance According to the $1^{\text {st }}$ fundamental law of thermodynamics, for humid air follows,

$$
\dot{m}_{\mathrm{SUP}} \cdot h_{\mathrm{MIA}}=\dot{Q}_{\mathrm{ac}}+\dot{m}_{\mathrm{SUP}} \cdot h_{\mathrm{AAC}} \cdot
$$

The enthalpy flow of ingoing air $\dot{m}_{\text {SUP }} \cdot h_{\text {MIA }}$ is equal to the enthalpy flow of outgoing air and the heat flow removed by the CRM. If the enthalpy of humid air is used according to Equation (2.10), one gets

$$
\dot{m}_{\mathrm{SUP}} \cdot\left(c_{\mathrm{air}} \cdot T_{\mathrm{MIA}}+\Delta h_{\mathrm{V}} \cdot Y_{\mathrm{MIA}}\right)=\dot{Q}_{\mathrm{ac}}+\dot{m}_{\mathrm{SUP}} \cdot\left(c_{\mathrm{air}} \cdot T_{\mathrm{AAC}}+\Delta h_{\mathrm{V}} \cdot Y_{\mathrm{AAC}}\right) .
$$

Electrical power consumption With the known coefficient of performance (COP) for the CRM, the electrical power consumption follows as

$$
P_{\mathrm{ac}}=\frac{\dot{Q}_{\mathrm{ac}}}{\mathrm{COP}_{\mathrm{ac}}}
$$

\section{Heat pump and electric heater}

When the CRM or the electric heater is heating the tram, the stated relationships of this subsection apply. In contrast to the cooling mode, equations are a bit simpler because condensation does not occur. Figure $5.4 \mathrm{c}$ shows a schematic sketch of the electric heater.

Energy balance It suffices to consider only dry air because the absolute humidity does not change

$$
\dot{m}_{\mathrm{SUP}} \cdot c_{\mathrm{air}} \cdot T_{\mathrm{AAC}}+\dot{Q}_{\mathrm{hp}}+\dot{Q}_{\mathrm{eh}}=\dot{m}_{\mathrm{SUP}} \cdot c_{\mathrm{air}} \cdot T_{\mathrm{SUP}} \cdot
$$

Electrical power consumption The COP allows calculating the electrical power consumption

$$
\begin{aligned}
& P_{\mathrm{hp}}=\frac{\dot{Q}_{\mathrm{hp}}}{\mathrm{COP}_{\mathrm{hp}}} \\
& P_{\mathrm{eh}}=\frac{\dot{Q}_{\mathrm{eh}}}{\mathrm{COP}_{\mathrm{eh}}} .
\end{aligned}
$$


The power consumption of the entire air conditioning system

The sum of the electrical power consumption is

$$
P_{\text {tot }}=P_{\text {fan }}+P_{\text {ac }}+P_{\mathrm{hp}}+P_{\text {eh }} .
$$

The modelling considers the electrical power consumption implicitly for other auxiliaries (for example valves and the controller). Auxiliaries necessary for running the unit are analysed along with the parts during power measurements.

\subsubsection{Optimisation problem}

This subsection transforms the control variable of the master controller in an energy-optimal way into the control variable of the air conditioning system by the slave controller. The special challenge of the slave controller is that most parts of the air conditioning system cannot be modelled continuously (as common in control engineering). So the controller must consider the individual states directly and has to satisfy many constraints. Since the objective is linear, the problem is a mixed integer linear problem (MILP). In general, it can be written as

$$
\begin{array}{cll}
\text { minimise } & c^{T} x+d & \\
\text { subject to } & \mathbf{A} x \leq b, & \mathbf{A} \in \mathbb{R}^{p \times m+n+r}, b \in \mathbb{R}^{p} \\
& \mathbf{A}_{\mathrm{eq}} x=b_{e q}, & \mathbf{A}_{\mathbf{e q}} \in \mathbb{R}^{q \times m+n+r}, b_{e q} \in \mathbb{R}^{q} \\
& x \in\left[x^{-}, x^{+}\right] & \\
& x=[\phi, \varphi, \delta]^{T}, & \phi \in \mathbb{R}^{m}, \varphi \in \mathbb{N}^{n}, \delta \in \mathbb{B}^{r}
\end{array}
$$

The optimisation problem follows from the equations described in Section 5.4.1.

Equation (5.50) describes the energy consumption of the air conditioning system and Section 5.3 explains how to calculate the master controller. The decision variables with the constraints are mentioned in the individual paragraphs and are summarised at the end. The slave controller can influence the following variables of the HVAC:

- the mass flow of outdoor air $\dot{m}_{\mathrm{ODA}}$,

- the mass flow of recirculation air $\dot{m}_{\mathrm{IDA}}$,

- the heat dissipated by the heat pump $\dot{Q}_{\mathrm{hp}}$,

- the heat absorbed by the CRM $\dot{Q}_{\mathrm{ac}}$ and

- the heat dissipated by the electric heater $\dot{Q}_{\mathrm{eh}}$.

The optimisation problem needs to satisfy the following constraints:

- constraints on comfort to guarantee the thermal comfort for the passengers,

- the state constraints on the air conditioning parts for preventing any damage,

- the conditions necessary for the FSM, preventing forbidden or impossible switching modes and

- the constraints on modes that describe the physical relations in each mode. 


\section{Constraints on comfort}

The controller also needs to consider various comfort constraints. [55] lists the considered constraints. Constraints apply to

- acceptable supply air temperature,

- minimum outdoor airflow,

- maximum air speed,

- and the maximum acceptable concentration of carbon dioxide in the indoor air.

The following paragraphs describe how these constraints are satisfied. [65] was used to derive the constraints.

Constraints on the supply air temperature There are upper and lower boundaries for the supply air temperature [60]. The minimum supply air temperature $T_{\text {SUP }}^{\mathrm{lb}} \geq 10^{\circ} \mathrm{C}$ is always lower than or equal to the minimum feasible indoor air temperature $T_{\mathrm{IDA}}^{\mathrm{lb}} \geq 12^{\circ} \mathrm{C}$. This is true if the outdoor air temperature is higher than $-14^{\circ} \mathrm{C}$. If that is not the case, the system is not (completely) controllable. $T_{\text {SUP }}^{\mathrm{lb}} \leq T_{\text {IDA }}^{\mathrm{lb}}$ must apply. The maximum supply air temperature $T_{\text {SUP }}^{\mathrm{ub}} \leq 60^{\circ} \mathrm{C}$ is always higher than the highest (feasible) indoor air temperature $T_{\mathrm{IDA}}^{\mathrm{ub}} \leq 40^{\circ} \mathrm{C} . T_{\text {SUP }}^{\mathrm{ub}} \geq T_{\mathrm{IDA}}^{\mathrm{ub}}$ must apply. So the inequalities follow

$$
\begin{aligned}
& -\varphi_{\mathrm{ODA}} \cdot \dot{m}_{\mathrm{ODA}}^{\mathrm{ub}} \cdot c_{\mathrm{air}} \cdot\left(T_{\mathrm{SUP}}^{\mathrm{ub}}-T_{\mathrm{IDA}}\right) \quad \ldots \\
& -\varphi_{\mathrm{IDA}} \cdot \dot{m}_{\mathrm{IDA}}^{\mathrm{ub}} \cdot c_{\mathrm{air}} \cdot\left(T_{\mathrm{SUP}}^{\mathrm{ub}}-T_{\mathrm{IDA}}\right) \leq \Delta h_{\mathrm{SUP}} \quad \text { and } \\
& \varphi_{\mathrm{ODA}} \cdot \dot{m}_{\mathrm{ODA}}^{\mathrm{ub}} \cdot c_{\mathrm{air}} \cdot\left(T_{\mathrm{SUP}}^{\mathrm{lb}}-T_{\mathrm{IDA}}\right) \quad \ldots \\
& +\varphi_{\mathrm{IDA}} \cdot \dot{m}_{\mathrm{IDA}}^{\mathrm{ub}} \cdot c_{\mathrm{air}} \cdot\left(T_{\mathrm{SUP}}^{\mathrm{lb}}-T_{\mathrm{IDA}}\right) \leq \Delta h_{\mathrm{SUP}} \cdot
\end{aligned}
$$

Both mass flows $\dot{m}_{\mathrm{ODA}}$ and $\dot{m}_{\mathrm{IDA}}$ were replaced by the matching terms $\varphi_{\mathrm{ODA}} \cdot \dot{m}_{\mathrm{ODA}}^{\mathrm{ub}}$ respectively $\varphi_{\mathrm{IDA}} \cdot \dot{m}_{\mathrm{IDA}}^{\mathrm{ub}} \cdot \varphi_{\mathrm{ODA}}, \varphi_{\mathrm{IDA}} \in[0,20] \subseteq \mathbb{N}$ applies and with $a^{u b}$ the maximum respectively $a^{l b}$ the minimum value of variable $a$ is denoted. In that case, the upper boundary of the mass flow of supply air and recirculation air is described. $\varphi_{\mathrm{ODA}}$ and $\varphi_{\mathrm{IDA}}$ represent the levels of the outdoor air respectively recirculation air.

Constraints on the supply air mass flow Similar to the supply air temperature, constraints on the supply air mass flow exist [60]. Thus, it is necessary to keep a minimum outdoor air mass flow. A lower bound therefore follows, and from the maximum mass flow rate of the fan an upper bound follows

$$
\begin{gathered}
\varphi_{\mathrm{ODA}} \cdot \dot{m}_{\mathrm{ODA}}^{\mathrm{ub}}+\varphi_{\mathrm{IDA}} \cdot \dot{m}_{\mathrm{IDA}}^{\mathrm{ub}} \leq \dot{m}_{\mathrm{SUP}}^{\mathrm{ub}} \quad \text { and } \\
-\varphi_{\mathrm{ODA}} \cdot \dot{m}_{\mathrm{ODA}}^{\mathrm{ub}}-\varphi_{\mathrm{IDA}} \cdot \dot{m}_{\mathrm{IDA}}^{\mathrm{ub}} \leq-\dot{m}_{\mathrm{SUP}}^{\mathrm{b}}
\end{gathered}
$$

The constraints for the maximum supply air mass flow $\dot{m}_{\mathrm{SUP}}^{\mathrm{ub}}$ are temperature dependent to satisfy the maximum airspeed in the train.

Constraints on the carbon dioxide concentration of the indoor air For the carbon dioxide concentration of the indoor air, no constraints exist according to EN 14750 [60], but a recommendation can be found in EN 13779 [88, Tab. A.10]. Under the assumption of category IDA 3 (moderate indoor 
air quality), an increase of the carbon dioxide concentration in the indoor air of $800 \mathrm{ppm}$ is feasible. Under the assumption that the outdoor air concentration $\sigma_{\mathrm{ODA}}$ is $300 \mathrm{ppm}$, the maximum feasible indoor air concentration is $\sigma_{\mathrm{IDA}}^{\mathrm{ub}}=1100$. The relationships follow from the balance equation (see Equation (5.36)). The balance can be reformulated to Equation (5.56)

$$
\dot{m}_{\mathrm{ODA}} \cdot\left(\sigma_{\mathrm{ODA}}-\sigma_{\mathrm{IDA}}\right)=\dot{m}_{\mathrm{SUP}} \cdot\left(\sigma_{\mathrm{SUP}}-\sigma_{\mathrm{IDA}}\right) .
$$

Equation (5.56) can substitute the right side of Equation (5.8). This yields Equation (5.57).

$$
\dot{m}_{\mathrm{ODA}} \cdot\left(\sigma_{\mathrm{ODA}}-\sigma_{\mathrm{IDA}}\right)=\Delta \sigma_{\mathrm{SUP}}
$$

The master controller satisfies the constraint $\Delta \sigma_{\text {SUP }} \leq 0$. Moreover, the concentration of carbon dioxide in the indoor air is higher than or equal to the outdoor air concentration. Through relaxation of the equation

$$
\dot{m}_{\mathrm{ODA}} \cdot \underbrace{\left(\sigma_{\mathrm{ODA}}-\sigma_{\mathrm{IDA}}\right)}_{\text {negative }}=\underbrace{\Delta \sigma_{\mathrm{SUP}}}_{\text {negative }}
$$

one gets the inequality

$$
\varphi_{\mathrm{ODA}} \cdot \dot{m}_{\mathrm{ODA}}^{\mathrm{ub}} \cdot\left(\sigma_{\mathrm{ODA}}-\sigma_{\mathrm{IDA}}\right) \leq \Delta \sigma_{\mathrm{SUP}}
$$

Equation (5.59) guarantees a minimum outdoor air mass flow into the tram.

\section{State constraints of the parts}

As in Section 5.3, where constraints were stated for the states, constraints are now defined for the parts of the air conditioning system.

Power constraints of the electric heater If turned on, a minimum and maximum power dissipation has to be considered for the electric heater

$$
\begin{aligned}
\varphi_{\mathrm{eh}}-n_{\mathrm{eh}}^{\mathrm{ub}} \cdot \delta_{\mathrm{eh}} \leq 0 & \text { and } \\
-\varphi_{\mathrm{eh}}+n_{\mathrm{eh}}^{\mathrm{lb}} \cdot \delta_{\mathrm{eh}} \leq 0 . &
\end{aligned}
$$

In which $\varphi_{\mathrm{eh}}$ is the used setting of the electric heater, $n_{\mathrm{eh}}^{\mathrm{lb}}$ the minimum respectively $n_{\mathrm{eh}}^{\mathrm{ub}}$ the maximum setting of the electric heater. $\delta_{\text {eh }}$ is a binary variable, which states if the electric heater is turned on. A setting unequal to zero $\varphi_{\mathrm{eh}} \neq 0$ will only be possible if the electric heater is switched on and $\delta_{\text {eh }}=1$ applies.

Rotational speed constraint of the compressor A minimum and maximum rotational speed must be considered if the compressor is on

$$
\begin{aligned}
\varphi_{\text {comp }}-n_{\text {comp }}^{\mathrm{ub}} \cdot \delta_{\text {comp }} \leq 0 \text { and } \\
-\varphi_{\text {comp }}+n_{\text {comp }}^{\mathrm{lb}} \cdot \delta_{\text {comp }} \leq 0 .
\end{aligned}
$$

$\varphi_{\text {comp }}$ is the chosen setting of the compressor. The explanations for the power constraints on the electric heater apply analogously. 
Constraints on the cooling cycle Constraints for the cooling cycle follow from the power balance. The spread (or absorbed) heat flow of the cooling cycle is proportional to the rotational speed of the compressor. When the compressor of the cooling cycle is running and the unit is in cooling mode, it follows that

$$
\phi_{\mathrm{ac}} \cdot \frac{\dot{Q}_{\mathrm{ac}}^{\mathrm{ub}}}{\mathrm{COP}_{\mathrm{ac}}}=f \cdot \varphi_{\mathrm{comp}}
$$

and in heat pump mode

$$
\phi_{\mathrm{hp}} \cdot \frac{\dot{Q}_{\mathrm{hp}}^{\mathrm{ub}}}{\mathrm{COP}_{\mathrm{hp}}}=f \cdot \varphi_{\mathrm{comp}} .
$$

applies. On the right side of Equation (5.64) and Equation (5.65) the power consumption of the compressor is written, in which $f$ describes the factor for calculating the power consumption from the compressor setting $\varphi_{\text {comp. }}$. The left side of both equations is also a power consumption, but in this case defined by the cooling power (that is the heating power) of the CRM. $\phi_{\mathrm{ac}}, \phi_{\mathrm{hp}} \in \mathbb{R}$ is a continuous auxiliary variable in the range of $[0,1]$. These equations under constraints can be reformulated as the following inequalities in cooling mode respectively in heating mode

$$
\begin{aligned}
& \phi_{\mathrm{ac}} \cdot \dot{Q}_{\mathrm{ac}}^{\mathrm{ub}}-\mathrm{COP}_{\mathrm{ac}} \cdot f \cdot \varphi_{\mathrm{comp}} \leq U_{\mathrm{ac}} \cdot\left(1-\delta_{\mathrm{CH}}\right) \text { and } \\
& \phi_{\mathrm{hp}} \cdot \dot{Q}_{\mathrm{ac}}^{\mathrm{ub}}-\mathrm{COP}_{\mathrm{hp}} \cdot f \cdot \varphi_{\mathrm{comp}} \leq U_{\mathrm{hp}} \cdot\left(1-\delta_{\mathrm{HC}}\right) .
\end{aligned}
$$

They can be transformed into

$$
\begin{gathered}
\phi_{\mathrm{ac}} \cdot \dot{Q}_{\mathrm{ac}}^{\mathrm{ub}}-\mathrm{COP}_{\mathrm{ac}} \cdot f \cdot \varphi_{\mathrm{comp}}+U_{\mathrm{ac}} \cdot \delta_{\mathrm{CH}} \leq U_{\mathrm{ac}} \quad \text { and } \\
\phi_{\mathrm{hp}} \cdot \dot{Q}_{\mathrm{ac}}^{\mathrm{ub}}-\mathrm{COP}_{\mathrm{hp}} \cdot f \cdot \varphi_{\mathrm{comp}}+U_{\mathrm{hp}} \cdot \delta_{\mathrm{HC}} \leq U_{\mathrm{hp}} .
\end{gathered}
$$

$\delta_{\mathrm{CH}}$ is a binary variable which is 1 when the CRM is in cooling mode or 0 otherwise. $\delta_{\mathrm{HC}}$ is 1 in the heating mode of the CRM. Equation (5.70) allows estimating the upper bound of the cooling mode $U_{\mathrm{ac}}$ and Equation (5.71) allows estimating the upper bound of the heat pump mode $U_{\mathrm{hp}}$

$$
\begin{aligned}
U_{\mathrm{ac}} & =\dot{Q}_{\mathrm{ac}}^{\mathrm{ub}} \quad \text { and } \\
U_{\mathrm{hp}} & =\dot{Q}_{\mathrm{hp}}^{\mathrm{ub}} .
\end{aligned}
$$

\section{Conditions for the FSM}

This subsection draws up the logic If-Else conditions as they appear in an FSM. The first part states the conditions of the cooling cycle and then the conditions of the four modes (heating, cooling, ventilation and dehumidifying).

Cooling cycle When the compressor is working, either the heat pump or the cooling mode of the CRM must be selected. Conversely, it follows that the compressor must be active when the heat pump or the cooling mode is active. In logic this can be expressed as

$$
\begin{aligned}
\mathscr{L}_{\mathrm{comp}} & \Rightarrow \mathscr{L}_{\mathrm{HC}} \vee \mathscr{L}_{\mathrm{CH}} \quad \text { and } \\
\left(\mathscr{L}_{\mathrm{HC}} \vee \mathscr{L}_{\mathrm{CH}}\right) & \Rightarrow \mathscr{L}_{\mathrm{comp}}
\end{aligned}
$$


But not both of the modes can be active at the same time

$$
\neg\left(\mathscr{L}_{\mathrm{HC}} \wedge \mathscr{L}_{\mathrm{CH}}\right) .
$$

The authors of [65] show how to transform logical expressions into inequalities. Equation (5.72), Equation (5.73) and Equation (5.74) therefore follow

$$
\begin{aligned}
\delta_{\mathrm{HC}}-\delta_{\text {comp }} & \leq 0, \\
\delta_{\mathrm{CH}}-\delta_{\text {comp }} & \leq 0, \\
\delta_{\text {comp }}-\delta_{\mathrm{HC}}-\delta_{\mathrm{CH}} & \leq 0 \text { and } \\
\delta_{\mathrm{CH}}+\delta_{\mathrm{HC}} & \leq 1,
\end{aligned}
$$

Besides that, the absorbed heat flow of the cooling cycle is equal to 0 , when the cooling respectively heat pump mode is selected. The logical relationships are

$$
\begin{aligned}
& \mathscr{L}_{\text {comp }} \wedge \mathscr{L}_{\mathrm{CH}} \Rightarrow \dot{Q}_{\mathrm{hp}} \quad \text { and } \\
& \mathscr{L}_{\text {comp }} \wedge \mathscr{L}_{\mathrm{HC}} \Rightarrow \dot{Q}_{\mathrm{ac}} .
\end{aligned}
$$

The inequalities describing these effects on the cooling mode are

$$
\begin{aligned}
\phi_{\mathrm{ac}}-\delta_{\text {comp }} & \leq 0 \text { and } \\
\phi_{\mathrm{ac}}-\delta_{\mathrm{CH}} & \leq 0,
\end{aligned}
$$

and for the heat pump mode

$$
\begin{gathered}
\phi_{\mathrm{hp}}-\delta_{\mathrm{comp}} \leq 0 \quad \text { and } \\
\phi_{\mathrm{hp}}-\delta_{\mathrm{HC}} \leq 0
\end{gathered}
$$

apply.

Heating If the HVAC is in the heating mode, either the compressor must be turned on and in this heat pump mode or the electric heater must be active

$$
\mathscr{L}_{\mathrm{H}} \Rightarrow\left(\mathscr{L}_{\text {comp }} \wedge \mathscr{L}_{\mathrm{HC}}\right) \vee \mathscr{L}_{\text {eh }} .
$$

The heat pump can only work if the compressor is also running

$$
\mathscr{L}_{\text {HC }} \Rightarrow \mathscr{L}_{\text {comp }}
$$

In addition, the mode heating is not active if the compressor is working but the cooling mode was selected

$$
\mathscr{L}_{\text {comp }} \wedge \mathscr{L}_{\mathrm{CH}} \Rightarrow \neg \mathscr{L}_{\mathrm{H}} \text {. }
$$


In this case, the dehumidification mode must be selected. From this, the inequalities follow

$$
\begin{gathered}
\delta_{\mathrm{H}}-\delta_{\text {comp }}-\delta_{\text {eh }} \leq 0 \\
\delta_{\mathrm{H}}-\delta_{\mathrm{HC}}-\delta_{\text {eh }} \leq 0 \text { and } \\
\delta_{\mathrm{HC}}-\delta_{\mathrm{comp}} \leq 0 .
\end{gathered}
$$

The $3^{\text {rd }}$ equation is identical with Equation (5.75) and was not numbered again.

Cooling If the HVAC is in cooling mode, the compressor must be switched on and in the cooling mode. It therefore follows that the electric heater must not be active

$$
\mathscr{L}_{\mathrm{C}} \Rightarrow \mathscr{L}_{\text {comp }} \wedge \mathscr{L}_{\mathrm{CH}} \wedge \neg\left(\mathscr{L}_{\text {eh }}\right)
$$

So the inequalities follow

$$
\begin{aligned}
\delta_{\mathrm{C}}-\delta_{\text {comp }} & \leq 0, \\
\delta_{\mathrm{C}}-\delta_{\mathrm{CH}} & \leq 0 \quad \text { and } \\
\delta_{\mathrm{C}}+\delta_{\text {eh }} & \leq 1 .
\end{aligned}
$$

Ventilation If the HVAC is in the ventilation mode, the compressor and the electric heater must be switched off

$$
\mathscr{L}_{\mathrm{V}} \Rightarrow \neg\left(\mathscr{L}_{\text {comp }}\right) \wedge \neg\left(\mathscr{L}_{\text {eh }}\right)
$$

So the inequalities follow

$$
\begin{array}{r}
\delta_{\mathrm{V}}+\delta_{\text {comp }} \leq 1 \\
\delta_{\mathrm{V}}+\delta_{\text {eh }} \leq 1
\end{array}
$$

Dehumidifying If the HVAC is in the dehumidification mode, the compressor must be switched on and in the cooling mode and the electric heater must be active

$$
\mathscr{L}_{\mathrm{D}} \Rightarrow \mathscr{L}_{\mathrm{comp}} \wedge \mathscr{L}_{\mathrm{CH}} \wedge \mathscr{L}_{\mathrm{eh}}
$$

So the inequalities follow

$$
\begin{aligned}
\delta_{\mathrm{D}}-\delta_{\text {comp }} & \leq 0, \\
\delta_{\mathrm{D}}-\delta_{\mathrm{CH}} & \leq 0 \quad \text { and } \\
\delta_{\mathrm{D}}-\delta_{\text {eh }} & \leq 0 .
\end{aligned}
$$




\section{Constraints on the air conditioning modes}

The functions of the HVAC can be directly used as modes

$$
\text { Mode }= \begin{cases}\delta_{\mathrm{V}}, & \text { Ventilation } \\ \delta_{\mathrm{H}}, & \text { Heating } \\ \delta_{\mathrm{C}}, & \text { Cooling } \\ \delta_{\mathrm{D}}, & \text { Dehumidification }\end{cases}
$$

Exactly one of these modes must be active. Consequently,

$$
\delta_{\mathrm{H}}+\delta_{\mathrm{C}}+\delta_{\mathrm{V}}+\delta_{\mathrm{D}}=1
$$

applies. The following paragraphs gather the conditions for the various modes.

Heating If air is heated, the energy supplied by the HVAC is equal to the necessary amount given by the master controller.

Energy balance in the heating mode The equation is

$$
h_{\mathrm{MIA}}+\sum_{i} \dot{Q}_{i}=h_{\mathrm{SUP}} \quad i \in H, H P .
$$

To the enthalpy of dry air applies that $h=\dot{m}_{\text {air }} \cdot c_{\text {air }} \cdot T$ is. Then,

$$
\dot{m}_{\mathrm{MIA}} \cdot c_{\mathrm{air}} \cdot T_{\mathrm{MIA}}+\sum_{i} \dot{Q}_{i}=\dot{m}_{\mathrm{SUP}} \cdot c_{\mathrm{air}} \cdot T_{\mathrm{SUP}} \quad i \in H, H P
$$

follows. According to Equation (5.33), it follows from the mass balance of the mixing chamber, it follows that mixing air can be replaced. This results in

$$
\dot{m}_{\mathrm{IDA}} \cdot c_{\mathrm{air}} \cdot T_{\mathrm{IDA}}+\dot{m}_{\mathrm{ODA}} \cdot c_{\mathrm{air}} \cdot T_{\mathrm{ODA}}+\sum_{i} \dot{Q}_{i}=\dot{m}_{\mathrm{SUP}} \cdot c_{\mathrm{air}} \cdot T_{\mathrm{SUP}} \quad i \in H, H P .
$$

Now the equation is divided by $c_{\text {air }} \cdot \dot{m}_{\text {SUP }}$. Thus,

$$
\frac{\dot{m}_{\mathrm{IDA}}}{\dot{m}_{\mathrm{SUP}}} \cdot T_{\mathrm{IDA}}+\frac{\dot{m}_{\mathrm{ODA}}}{\dot{m}_{\mathrm{SUP}}} \cdot T_{\mathrm{ODA}}+\frac{\sum_{i} \dot{Q}_{i}}{\dot{m}_{\mathrm{SUP}} \cdot c_{\mathrm{air}}}=T_{\mathrm{SUP}} \quad i \in H, H P
$$

follows. The expressions of the mass flows are substituted by the outdoor air ratio according to Equation (5.37) and the recirculation air ratio according to Equation (5.38). One gets

$$
\alpha \cdot T_{\mathrm{ODA}}-\alpha \cdot T_{\mathrm{IDA}}+\frac{\sum_{i} \dot{Q}_{i}}{\dot{m}_{\mathrm{SUP}} \cdot c_{\mathrm{air}}}=T_{\mathrm{SUP}}-T_{\mathrm{IDA}} \quad i \in H, H P .
$$

The outdoor air ratio is substituted back and the equation is multiplied by $c_{\text {air }} \cdot \dot{m}_{\mathrm{SUP}}$

$$
\dot{m}_{\mathrm{ODA}} \cdot c_{\mathrm{air}} \cdot\left(T_{\mathrm{ODA}}-T_{\mathrm{IDA}}\right)+\sum_{i} \dot{Q}_{i}=\Delta h_{\mathrm{SUP}} \quad i \in H, H P,
$$


follows, when the manipulation variable of the master controller is applied to Equation (5.6). The heat pump and the electric heater supply the necessary heat flow in heating. The dissipated heat flow of the electric heater is given as

$$
\dot{Q}_{\mathrm{eh}}=\phi_{\mathrm{eh}} \cdot \dot{Q}_{\mathrm{eh}}^{\mathrm{ub}}
$$

For the dissipated heat flow of the heat pump

$$
\dot{Q}_{\mathrm{hp}}=\phi_{\mathrm{hp}} \cdot \dot{Q}_{\mathrm{hp}}^{\mathrm{ub}}
$$

follows analogously. The energy balance is therefore

$$
\varphi_{\mathrm{ODA}} \cdot \dot{m}_{\mathrm{ODA}}^{\mathrm{ub}} \cdot\left(T_{\mathrm{ODA}}-T_{\mathrm{IDA}}\right)+\phi_{\mathrm{hp}} \cdot \frac{\dot{Q}_{\mathrm{hp}}^{\mathrm{ub}}}{c_{\mathrm{air}}}+\phi_{\mathrm{eh}} \cdot \frac{\dot{Q}_{\mathrm{eh}}^{\mathrm{ub}}}{c_{\mathrm{air}}}=\frac{\Delta h_{\mathrm{SUP}}}{c_{\mathrm{air}}} .
$$

Inequalities in the heating mode The energy balance will only be valid if the HVAC is in the heating mode. Thus, the two inequalities

$$
\begin{gathered}
\varphi_{\mathrm{ODA}} \cdot \dot{m}_{\mathrm{ODA}}^{\mathrm{ub}} \cdot\left(T_{\mathrm{ODA}}-T_{\mathrm{IDA}}\right)+\phi_{\mathrm{hp}} \cdot \frac{\dot{Q}_{\mathrm{hp}}^{\mathrm{ub}}}{c_{\mathrm{air}}} \ldots \\
+\phi_{\mathrm{eh}} \cdot \frac{\dot{Q}_{\mathrm{eh}}^{\mathrm{ub}}}{c_{\mathrm{air}}}+U_{\mathrm{H}} \cdot \delta_{\mathrm{H}} \leq U_{\mathrm{H}}+\frac{\Delta h_{\mathrm{SUP}}}{c_{\mathrm{air}}} \text { and } \\
-\varphi_{\mathrm{ODA}} \cdot \dot{m}_{\mathrm{ODA}}^{\mathrm{ub}} \cdot\left(T_{\mathrm{ODA}}-T_{\mathrm{IDA}}\right)-\phi_{\mathrm{hp}} \cdot \frac{\dot{Q}_{\mathrm{hp}}^{\mathrm{ub}}}{c_{\mathrm{air}}} \ldots \\
-\phi_{\mathrm{eh}} \cdot \frac{\dot{Q}_{\mathrm{eh}}^{\mathrm{ub}}}{c_{\mathrm{air}}}-L_{\mathrm{H}} \cdot \delta_{\mathrm{H}} \leq-L_{\mathrm{H}}-\frac{\Delta h_{\mathrm{SUP}}}{c_{\mathrm{air}}}
\end{gathered}
$$

follow. The newly introduced variables $U_{\mathrm{H}}$ and $L_{\mathrm{H}}$ are bounds of Equation (5.111), in which $U_{\mathrm{H}}$ is the upper and $L_{\mathrm{H}}$ the lower bound. Those bounds follow from worst case estimations. They are given as

$$
\begin{aligned}
& L_{\mathrm{H}}=\dot{m}_{\mathrm{ODA}}^{\mathrm{ub}} \cdot\left(T_{\mathrm{ODA}}^{\mathrm{lb}}-T_{\mathrm{IDA}}^{\mathrm{ub}}\right)+\frac{\dot{Q}_{\mathrm{hp}}^{\mathrm{lb}}}{c_{\mathrm{air}}}+\frac{\dot{Q}_{\mathrm{eh}}^{\mathrm{lb}}}{c_{\mathrm{air}}}-\frac{\Delta h_{\mathrm{SUP}}^{\mathrm{ub}}}{c_{\mathrm{air}}} \text { and } \\
& U_{\mathrm{H}}=\dot{m}_{\mathrm{ODA}}^{\mathrm{ub}} \cdot\left(T_{\mathrm{ODA}}^{\mathrm{ub}}-T_{\mathrm{IDA}}^{\mathrm{lb}}\right)+\frac{\dot{Q}_{\mathrm{hp}}^{\mathrm{ub}}}{c_{\mathrm{air}}}+\frac{\dot{Q}_{\mathrm{eh}}^{\mathrm{ub}}}{c_{\mathrm{air}}}-\frac{\Delta h_{\mathrm{SUP}}^{\mathrm{lb}}}{c_{\mathrm{air}}} .
\end{aligned}
$$

This method guarantees that Equation (5.111) is valid in all other modes, too.

Cooling The cooling mode is more difficult to handle than the heating mode. The master controller satisfies both manipulated variables $\Delta h_{\mathrm{SUP}}$ and $\Delta Y_{\mathrm{SUP}}$ by one actuator (the CRM). 
Energy balance in the cooling mode The energy balance of dry air is

$$
h_{\mathrm{MIA}}-\dot{Q}_{\mathrm{ac}, \mathrm{sen}}=h_{\mathrm{SUP}} \text {. }
$$

The mass balance of water is

$$
Y_{\mathrm{MIA}}-\dot{m}_{\mathrm{ac}}=Y_{\mathrm{SUP}},
$$

in which $\dot{m}_{\mathrm{ac}}$ is the water removed by the HVAC. Equation (5.116) applies after some transformations

$$
\varphi_{\mathrm{ODA}} \cdot \dot{m}_{\mathrm{ODA}}^{\mathrm{ub}} \cdot c_{\mathrm{air}} \cdot\left(T_{\mathrm{ODA}}-T_{\mathrm{IDA}}\right)-\dot{Q}_{\mathrm{ac}, \mathrm{sen}}=\Delta h_{\mathrm{SUP}}
$$

and with Equation (5.117)

$$
\varphi_{\mathrm{ODA}} \cdot \dot{m}_{\mathrm{ODA}}^{\mathrm{ub}} \cdot\left(Y_{\mathrm{ODA}}-Y_{\mathrm{IDA}}\right)-m_{\mathrm{ac}}=\Delta Y_{\mathrm{SUP}}
$$

follows. The enthalpy of humid air (see Equation (2.10)) can be calculated from the sum of enthalpies of dry air and water. Both Equation (5.118) and Equation (5.119) are now in the same unit and can be added up. One gets

$$
\begin{gathered}
\varphi_{\mathrm{ODA}} \cdot \dot{m}_{\mathrm{ODA}}^{\mathrm{ub}} \cdot\left[c_{\mathrm{air}} \cdot\left(T_{\mathrm{ODA}}-T_{\mathrm{IDA}}\right)+\Delta h_{\mathrm{V}} \cdot\left(Y_{\mathrm{ODA}}-Y_{\mathrm{IDA}}\right)\right] \ldots \\
-\phi_{\mathrm{ac}} \cdot \dot{Q}_{\mathrm{ac}}^{\mathrm{ub}}=\Delta h_{\mathrm{SUP}}+\Delta h_{\mathrm{V}} \cdot \Delta Y_{\mathrm{SUP}},
\end{gathered}
$$

where the absorbed heat flow of the cooling cycle $\phi_{\mathrm{ac}} \cdot \dot{Q}_{\mathrm{ac}}^{\mathrm{ub}}$ replaces the sensible heat flow $\dot{Q}_{\mathrm{ac}, \mathrm{sen}}$ and the latent heat flow $\Delta h_{\mathrm{V}} \cdot \dot{m}_{\mathrm{ac}}$. Another way to gain this relation starts from Equation (5.45), in which the mixing air according to Equation (5.34) and Equation (5.35) is replaced, so that Equation (5.6) and Equation (5.7) can substitute them.

Inequalities in the cooling mode The energy balance in cooling will only be valid if the HVAC is in the cooling mode

$$
\begin{array}{r}
\varphi_{\mathrm{ODA}} \cdot \dot{m}_{\mathrm{ODA}}^{\mathrm{ub}} \cdot\left[c_{\mathrm{air}} \cdot\left(T_{\mathrm{ODA}}-T_{\mathrm{IDA}}\right)+\Delta h_{\mathrm{V}} \cdot\left(Y_{\mathrm{ODA}}-Y_{\mathrm{IDA}}\right)\right] \ldots \\
-\phi_{\mathrm{ac}} \cdot \dot{Q}_{\mathrm{ac}}^{\mathrm{ub}}+U_{\mathrm{C}} \cdot \delta_{\mathrm{C}} \leq U_{\mathrm{C}}+\Delta h_{\mathrm{SUP}}+\Delta h_{\mathrm{V}} \cdot \Delta Y_{\mathrm{SUP}} \\
-\varphi_{\mathrm{ODA}} \cdot \dot{m}_{\mathrm{ODA}}^{\mathrm{ub}} \cdot\left[c_{\mathrm{air}} \cdot\left(T_{\mathrm{ODA}}-T_{\mathrm{IDA}}\right)+\Delta h_{\mathrm{V}} \cdot\left(Y_{\mathrm{ODA}}-Y_{\mathrm{IDA}}\right)\right] \ldots \\
+\phi_{\mathrm{ac}} \cdot \dot{Q}_{\mathrm{ac}}^{\mathrm{ub}}-L_{\mathrm{C}} \cdot \delta_{\mathrm{C}} \leq-L_{\mathrm{C}}-\Delta h_{\mathrm{SUP}}-\Delta h_{\mathrm{V}} \cdot \Delta Y_{\mathrm{SUP}}
\end{array}
$$

The following relations apply to the upper bound $U_{\mathrm{C}}$ respectively lower bound $L_{\mathrm{C}}$

$$
\begin{aligned}
& L_{\mathrm{C}}=\dot{m}_{\mathrm{ODA}}^{\mathrm{ub}} \cdot\left[c_{\mathrm{air}} \cdot\left(T_{\mathrm{ODA}}^{\mathrm{lb}}-T_{\mathrm{IDA}}^{\mathrm{ub}}\right)+\Delta h_{\mathrm{V}} \cdot\left(Y_{\mathrm{ODA}}^{\mathrm{lb}}-Y_{\mathrm{IDA}}^{\mathrm{ub}}\right)\right] \ldots \\
& -\dot{Q}_{\mathrm{ac}}^{\mathrm{ub}}-\Delta h_{\mathrm{SUP}}^{\mathrm{ub}}-\Delta h_{\mathrm{V}} \cdot \Delta Y_{\mathrm{SUP}}^{\mathrm{ub}} \text { and } \\
& U_{\mathrm{C}}=\dot{m}_{\mathrm{ODA}}^{\mathrm{ub}} \cdot\left[c_{\mathrm{air}} \cdot\left(T_{\mathrm{ODA}}^{\mathrm{ub}}-T_{\mathrm{IDA}}^{\mathrm{lb}}\right)+\Delta h_{\mathrm{V}} \cdot\left(Y_{\mathrm{ODA}}^{\mathrm{ub}}-Y_{\mathrm{IDA}}^{\mathrm{lb}}\right)\right] \ldots \\
& -\dot{Q}_{\mathrm{ac}}^{\mathrm{ub}}-\Delta h_{\mathrm{SUP}}^{\mathrm{lb}}-\Delta h_{\mathrm{V}} \cdot \Delta Y_{\mathrm{SUP}}^{\mathrm{lb}} \text {. }
\end{aligned}
$$

Ventilation The presented method uses a temperature control in the ventilation mode, so that, the energy balance is similar as in the cooling and the heating mode. 
Energy balance in the ventilation mode The equation will follow similarly to the energy balance in heating (see Equation (5.111)) if the sum of dissipated heat flows is equal to 0. One gets Equation (5.125)

$$
\varphi_{\mathrm{ODA}} \cdot \dot{m}_{\mathrm{ODA}}^{\mathrm{ub}} \cdot\left(T_{\mathrm{ODA}}-T_{\mathrm{IDA}}\right)=\frac{\Delta h_{\mathrm{SUP}}}{c_{\mathrm{air}}}
$$

Inequalities in the ventilation mode The energy balance in ventilation will only be valid if the HVAC is in the ventilation mode

$$
\begin{gathered}
\varphi_{\mathrm{ODA}} \cdot \dot{m}_{\mathrm{ODA}}^{\mathrm{ub}} \cdot\left(T_{\mathrm{ODA}}-T_{\mathrm{IDA}}\right)+U_{\mathrm{V}} \cdot \delta_{\mathrm{V}} \leq U_{\mathrm{V}}+\frac{\Delta h_{\mathrm{SUP}}}{c_{\mathrm{air}}}, \\
-\varphi_{\mathrm{ODA}} \cdot \dot{m}_{\mathrm{ODA}}^{\mathrm{ub}} \cdot\left(T_{\mathrm{ODA}}-T_{\mathrm{IDA}}\right)-L_{\mathrm{V}} \cdot \delta_{\mathrm{V}} \leq-L_{\mathrm{V}}-\frac{\Delta h_{\mathrm{SUP}}}{c_{\mathrm{air}}} .
\end{gathered}
$$

The following relationships apply to the upper bound $U_{\mathrm{V}}$ and lower bound $L_{\mathrm{V}}$

$$
\begin{aligned}
& L_{\mathrm{V}}=\dot{m}_{\mathrm{ODA}}^{\mathrm{ub}} \cdot\left(T_{\mathrm{ODA}}^{\mathrm{lb}}-T_{\mathrm{IDA}}^{\mathrm{ub}}\right)-\frac{\Delta h_{\mathrm{SUP}}^{\mathrm{ub}}}{c_{\mathrm{air}}} \text { and } \\
& U_{\mathrm{V}}=\dot{m}_{\mathrm{ODA}}^{\mathrm{ub}} \cdot\left(T_{\mathrm{ODA}}^{\mathrm{ub}}-T_{\mathrm{IDA}}^{\mathrm{lb}}\right)-\frac{\Delta h_{\mathrm{SUP}}^{\mathrm{lb}}}{c_{\mathrm{air}}}
\end{aligned}
$$

Dehumidification In this mode, both temperature and humidity control are possible simultaneously, but this needs a high energy consumption.

Energy balance in the dehumidification mode In dehumidification the energy balance applies analogous to cooling (see Equation (5.120)), but, in addition, the electric heater can also produce the heat flow

$$
\begin{array}{r}
\varphi_{\mathrm{ODA}} \cdot \dot{m}_{\mathrm{ODA}}^{\mathrm{ub}} \cdot\left[c_{\mathrm{air}} \cdot\left(T_{\mathrm{ODA}}-T_{\mathrm{IDA}}\right)+\Delta h_{\mathrm{V}} \cdot\left(Y_{\mathrm{ODA}}-Y_{\mathrm{IDA}}\right)\right] \ldots \\
-\phi_{\mathrm{ac}} \cdot \dot{Q}_{\mathrm{ac}}^{\mathrm{ub}}+\phi_{\mathrm{eh}} \cdot \dot{Q}_{\mathrm{eh}}^{\mathrm{ub}}=\Delta h_{\mathrm{SUP}}+\Delta h_{\mathrm{V}} \cdot \Delta Y_{\mathrm{SUP}} .
\end{array}
$$

Inequalities in the dehumidification mode The energy balance in dehumidification will only be valid if the HVAC is in the dehumidifying mode

$$
\begin{gathered}
\varphi_{\mathrm{ODA}} \cdot \dot{m}_{\mathrm{ODA}}^{\mathrm{ub}} \cdot\left[c_{\mathrm{air}} \cdot\left(T_{\mathrm{ODA}}-T_{\mathrm{IDA}}\right)+\Delta h_{\mathrm{V}} \cdot\left(Y_{\mathrm{ODA}}-Y_{\mathrm{IDA}}\right)\right] \ldots \\
-\phi_{\mathrm{ac}} \cdot \dot{Q}_{\mathrm{ac}}^{\mathrm{ub}}+\phi_{\mathrm{eh}} \cdot \dot{Q}_{\mathrm{eh}}^{\mathrm{ub}}+U_{\mathrm{D}} \cdot \delta_{\mathrm{D}} \leq U_{\mathrm{D}}+\Delta h_{\mathrm{SUP}}+\Delta h_{\mathrm{V}} \cdot \Delta Y_{\mathrm{SUP}} \text { and } \\
-\varphi_{\mathrm{ODA}} \cdot \dot{m}_{\mathrm{ODA}}^{\mathrm{ub}} \cdot\left[c_{\mathrm{air}} \cdot\left(T_{\mathrm{ODA}}-T_{\mathrm{IDA}}\right)+\Delta h_{\mathrm{V}} \cdot\left(Y_{\mathrm{ODA}}-Y_{\mathrm{IDA}}\right)\right] \ldots \\
+\phi_{\mathrm{ac}} \cdot \dot{Q}_{\mathrm{ac}}^{\mathrm{ub}}-\phi_{\mathrm{eh}} \cdot \dot{Q}_{\mathrm{eh}}^{\mathrm{ub}}-U_{\mathrm{D}} \cdot \delta_{\mathrm{D}} \leq-L_{\mathrm{D}}-\Delta h_{\mathrm{SUP}}-\Delta h_{\mathrm{V}} \cdot \Delta Y_{\mathrm{SUP}} .
\end{gathered}
$$


The following relationships apply to the upper $U_{\mathrm{D}}$ and lower bound $L_{\mathrm{D}}$

$$
\begin{array}{r}
L_{\mathrm{D}}=\dot{m}_{\mathrm{ODA}}^{\mathrm{ub}} \cdot\left[c_{\mathrm{air}} \cdot\left(T_{\mathrm{ODA}}^{\mathrm{lb}}-T_{\mathrm{IDA}}^{\mathrm{ub}}\right)+\Delta h_{\mathrm{V}} \cdot\left(Y_{\mathrm{ODA}}^{\mathrm{lb}}-Y_{\mathrm{IDA}}^{\mathrm{ub}}\right)\right] \ldots \\
-\dot{Q}_{\mathrm{ac}}^{\mathrm{ub}}+\dot{Q}_{\mathrm{eh}}^{\mathrm{lb}}-\Delta h_{\mathrm{SUP}}^{\mathrm{ub}}-\Delta h_{\mathrm{V}} \cdot \Delta Y_{\mathrm{SUP}}^{\mathrm{ub}} \text { and } \\
U_{\mathrm{D}}=\dot{m}_{\mathrm{ODA}}^{\mathrm{ub}} \cdot\left[c_{\mathrm{air}} \cdot\left(T_{\mathrm{ODA}}^{\mathrm{ub}}-T_{\mathrm{IDA}}^{\mathrm{lb}}\right)+\Delta h_{\mathrm{V}} \cdot\left(Y_{\mathrm{ODA}}^{\mathrm{ub}}-Y_{\mathrm{IDA}}^{\mathrm{lb}}\right)\right] \ldots \\
-\dot{Q}_{\mathrm{ac}}^{\mathrm{ub}}+\dot{Q}_{\mathrm{eh}}^{\mathrm{ub}}-\Delta h_{\mathrm{SUP}}^{\mathrm{lb}}-\Delta h_{\mathrm{V}} \cdot \Delta Y_{\mathrm{SUP}}^{\mathrm{lb}} \cdot
\end{array}
$$

\section{Definition of the optimisation variable}

The decision variable $x$ of the optimisation problem follows from the continuous, integer and binary variables of

$$
x=\left[\phi_{\mathrm{ac}}, \phi_{\mathrm{hp}}, \varphi_{\mathrm{eh}}, \varphi_{\mathrm{comp}}, \varphi_{\mathrm{ODA}}, \varphi_{\mathrm{IDA}}, \delta_{\mathrm{H}}, \delta_{\mathrm{V}}, \delta_{\mathrm{C}}, \delta_{\mathrm{D}}, \delta_{\mathrm{comp}}\right]^{T} .
$$

\section{Cost function}

Costs follow from the energy consumption of the parts and Equation (5.50) sums up the power consumption of the electric heater, the compressor and the supply air fan. For the individual switchable parts follows

$$
\begin{aligned}
P_{\mathrm{eh}} & =\varphi_{\mathrm{eh}} \cdot \dot{Q}_{\mathrm{eh}}^{\mathrm{ub}}, \\
P_{\mathrm{comp}} & =f \cdot \varphi_{\mathrm{comp}} \text { and } \\
P_{\mathrm{fan}} & =k_{\text {venti }} \cdot\left(\varphi_{\mathrm{ODA}}+\varphi_{\mathrm{IDA}}\right)+d_{\text {venti }} .
\end{aligned}
$$

It is of not interest whether the heat pump $P_{\mathrm{hp}}$ or the cooling mode $P_{\mathrm{ac}}$ causes the energy consumption of the compressor. $P_{\text {comp }}$ summarises both and so the linear cost function follows

$$
\begin{aligned}
c^{T} & =\left[0,0, k_{H C}, f, k_{\mathrm{venti}} \cdot \dot{m}_{\mathrm{ODA}}^{\mathrm{ub}}, k_{\mathrm{venti}} \cdot \dot{m}_{\mathrm{IDA}}^{\mathrm{ub}}, 0,0,0,0,0,0,0\right] \\
d & =d_{\text {venti }}
\end{aligned}
$$

When optimising for $x$, the optimisation can neglect $d$ because it does not influence the decision variable. If the value of the cost function is used further, the term can be added later.

\subsubsection{Simplified optimisation problem}

Although drafting the optimisation problem presented in Section 5.4.2 already provides good results, sometimes there are difficulties in finding a feasible solution. Especially the split of the compressor power into cooling and heat pump power leads to problems. In addition, decreasing the number of variables reduces the calculation time. A new choice of $x$ achieves this improvement.

Figure 5.5 schematically represents the idea of this simplification. Based on the modes of Section 5.4.2, the other binary variables are analysed. There are no changes to the modes of cooling, ventilation and dehumidification. In the heating mode, two possibilities arise for the electric heater because it can be switched on or off. These two modes are split further by running the compressor. One gets three new modes from the old heating mode. Switching off the electric heater 


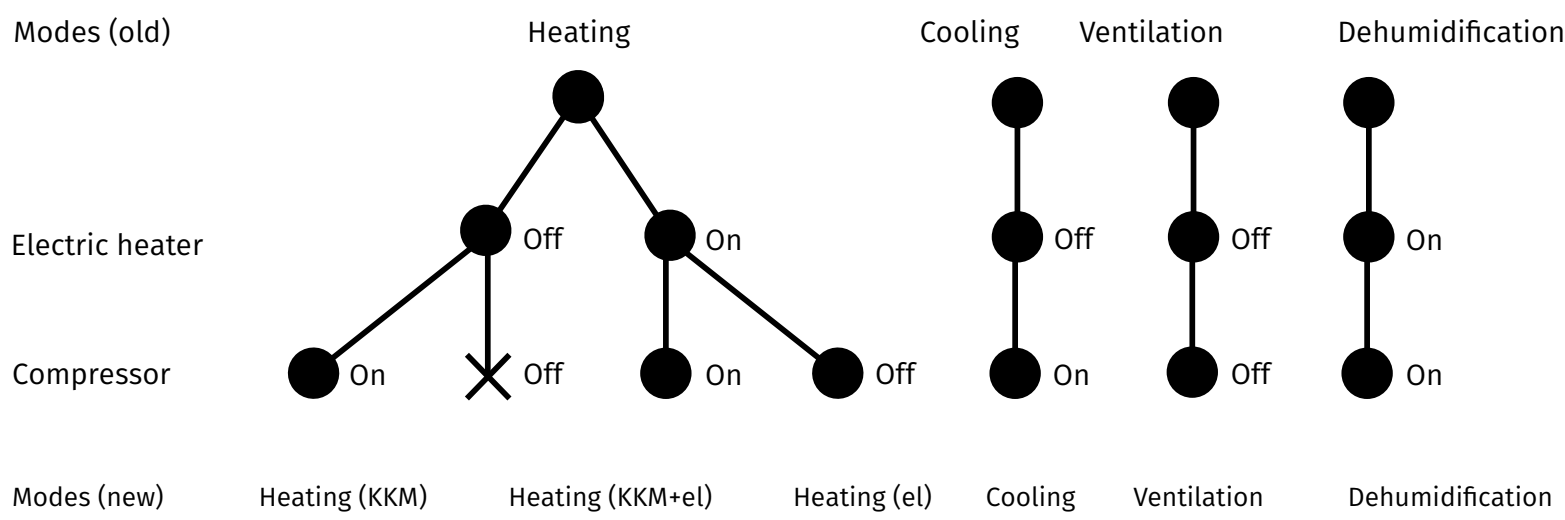

Figure 5.5. - Simplifying the optimisation problem

and the compressor at the same time is not possible in heating, this is the ventilation mode. In each of the new heating modes a different method is used: only the heat pump, the electric heater together with the heat pump together and only the electric heater. This results in six new modes. Based on this idea, the redefined $x$ is

$$
x=\left[\delta_{\mathrm{H}}, \delta_{\mathrm{E}}, \delta_{\mathrm{B}}, \delta_{\mathrm{C}}, \delta_{\mathrm{V}}, \delta_{\mathrm{D}}, \varphi_{\mathrm{eh}}, \varphi_{\mathrm{comp}}, \varphi_{\mathrm{ODA}}, \varphi_{\mathrm{IDA}}\right] .
$$

Due to these new modes, the split of the compressor power by the variables $\delta_{\mathrm{CH}}$ respectively $\delta_{\mathrm{HC}}$ can be dropped. In the modes of heating (el), heating $(e l+C R M)$ and dehumidification the electric heater is always switched on and in all other modes off. $\delta_{\text {eh }}$ can therefore be dropped as well. In addition, the continuous variables $\phi_{\mathrm{ac}}$ and $\phi_{\mathrm{hp}}$ can also be dropped because of the changed modes. This results in a new objective of the new form

$$
c=\left[0,0,0,0,0,0, k_{H C}, f, k_{\text {venti }} \cdot m_{O D A}^{+}, k_{\text {venti }} \cdot m_{I D A}^{+}\right]^{T} .
$$

The optimisation problem has become an integer linear problem (ILP) because it no longer contains continuous variables.

\section{Comfort conditions}

The constraints described in Section 5.4.2 are still valid here. The state constraints of the parts must be adjusted to the changed variables.

\section{State constraints of the parts}

The power constraint of the electric heater and the rotational speed constraint of the compressor must be adapted.

Power constraints of the electric heater The electric heater must satisfy the minimum and maximum power dissipation if it is used. This is valid in the modes of heating (el), heating (el+CRM) and 
dehumidification.

$$
\begin{aligned}
\varphi_{\mathrm{eh}}+n_{\mathrm{eh}}^{\mathrm{ub}} \cdot \delta_{i} & \leq 2 \cdot n_{\mathrm{eh}}^{\mathrm{ub}} \quad i \in E, B, D, \\
-\varphi_{\mathrm{eh}}+n_{\mathrm{eh}}^{\mathrm{lb}} \cdot \delta_{i} \leq 0 \quad i \in E, B, D . &
\end{aligned}
$$

Rotational speed constraint of the compressor If used, the compressor must satisfy the maximum and the minimum rotational speed. It is one of the modes of heating (CRM), heating $(C R M+e l)$, cooling and dehumidification

$$
\begin{gathered}
\varphi_{\mathrm{comp}}+n_{\mathrm{comp}}^{\mathrm{ub}} \cdot \delta_{i} \leq 2 \cdot n_{\mathrm{comp}}^{\mathrm{ub}} \quad i \in H, B, K, D, \\
-\varphi_{\mathrm{comp}}+n_{\mathrm{comp}}^{\mathrm{lb}} \cdot \delta_{i} \leq 0 \quad i \in H, B, K, D .
\end{gathered}
$$

Requirements of modes

As described above, the mode follows from a set of six possibilities (see Figure 5.5).

$$
\text { Mode }= \begin{cases}\delta_{\mathrm{H}}, & \text { Heating }(\mathrm{CRM}) \\ \delta_{\mathrm{E}}, & \text { Heating (el) } \\ \delta_{\mathrm{B}}, & \text { Heating }(\mathrm{CRM}+\mathrm{el}) \\ \delta_{\mathrm{C}}, & \text { Cooling } \\ \delta_{\mathrm{V}}, & \text { Ventilation } \\ \delta_{\mathrm{D}}, & \text { Dehumidification }\end{cases}
$$

Exactly one of these modes must be active. It follows

$$
\delta_{\mathrm{H}}+\delta_{\mathrm{E}}+\delta_{\mathrm{B}}+\delta_{\mathrm{C}}+\delta_{\mathrm{V}}+\delta_{\mathrm{D}}=1 .
$$

The following paragraphs derive the conditions of the individual modes.

Heating (CRM+el) To get the energy balance in heating $(C R M+e l)$, one has to replace the term $\phi_{\mathrm{hp}} \cdot \dot{Q}_{\mathrm{hp}}$ in Equation (5.111) with the help of Equation (5.65). Thus, the energy balance follows

$$
\varphi_{\mathrm{ODA}} \cdot \dot{m}_{\mathrm{ODA}}^{\mathrm{ub}} \cdot\left(T_{\mathrm{ODA}}-T_{\mathrm{IDA}}\right)+\varphi_{\mathrm{comp}} \cdot \frac{f \cdot \mathrm{COP}_{\mathrm{hp}}}{c_{\mathrm{air}}}+\varphi_{\mathrm{eh}} \cdot \frac{\dot{Q}_{\mathrm{eh}}^{\mathrm{ub}}}{c_{\mathrm{air}}}=\frac{\Delta h_{\mathrm{SUP}}}{c_{\mathrm{air}}}
$$


The two inequalities

$$
\begin{array}{r}
\varphi_{\mathrm{ODA}} \cdot \dot{m}_{\mathrm{ODA}}^{\mathrm{ub}} \cdot\left(T_{\mathrm{ODA}}-T_{\mathrm{IDA}}\right)+\varphi_{\mathrm{comp}} \cdot \frac{f \cdot \mathrm{COP}_{\mathrm{hp}}}{c_{\mathrm{air}}} \ldots \\
+\phi_{\mathrm{eh}} \cdot \frac{\dot{Q}_{\mathrm{eh}}^{\mathrm{ub}}}{c_{\mathrm{air}}}+U_{\mathrm{B}} \cdot \delta_{\mathrm{B}} \leq U_{\mathrm{B}}+\frac{\Delta h_{\mathrm{SUP}}}{c_{\mathrm{air}}} \text { and } \\
-\varphi_{\mathrm{ODA}} \cdot \dot{m}_{\mathrm{ODA}}^{\mathrm{ub}} \cdot\left(T_{\mathrm{ODA}}-T_{\mathrm{IDA}}\right)-\varphi_{\mathrm{comp}} \cdot \frac{f \cdot \mathrm{COP}_{\mathrm{hp}}}{c_{\mathrm{air}}} \ldots \\
-\phi_{\mathrm{eh}} \cdot \frac{\dot{Q}_{\mathrm{eh}}^{\mathrm{ub}}}{c_{\mathrm{air}}}-L_{\mathrm{B}} \cdot \delta_{\mathrm{B}} \leq-L_{\mathrm{B}}-\frac{\Delta h_{\mathrm{SUP}}}{c_{\mathrm{air}}} .
\end{array}
$$

follow from Equation (5.148). The values of $U_{\mathrm{B}}$ und $L_{\mathrm{B}}$ are identical with the values of $U_{\mathrm{H}}$ and $L_{\mathrm{H}}$ from Equation (5.114).

Heating (CRM) If heating is only possible with the CRM, Equation (5.114) simplifies to

$$
\varphi_{\mathrm{ODA}} \cdot \dot{m}_{\mathrm{ODA}}^{\mathrm{ub}} \cdot\left(T_{\mathrm{ODA}}-T_{\mathrm{IDA}}\right)+\varphi_{\mathrm{comp}} \cdot \frac{f \cdot \mathrm{COP}_{\mathrm{hp}}}{c_{\mathrm{air}}}=\frac{\Delta h_{\mathrm{SUP}}}{c_{\mathrm{air}}},
$$

from which both inequalities

$$
\begin{array}{r}
\varphi_{\mathrm{ODA}} \cdot \dot{m}_{\mathrm{ODA}}^{\mathrm{ub}} \cdot\left(T_{\mathrm{ODA}}-T_{\mathrm{IDA}}\right)+\varphi_{\mathrm{comp}} \cdot \frac{f \cdot \mathrm{COP}_{\mathrm{hp}}}{c_{\mathrm{air}}} \ldots \\
+U_{\mathrm{H}} \cdot \delta_{\mathrm{H}} \leq U_{\mathrm{H}}+\frac{\Delta h_{\mathrm{SUP}}}{c_{\mathrm{air}}} \text { and } \\
-\varphi_{\mathrm{ODA}} \cdot \dot{m}_{\mathrm{ODA}}^{\mathrm{ub}} \cdot\left(T_{\mathrm{ODA}}-T_{\mathrm{IDA}}\right)-\varphi_{\mathrm{comp}} \cdot \frac{f \cdot \mathrm{COP}_{\mathrm{hp}}}{c_{\mathrm{air}}} \ldots \\
-L_{\mathrm{H}} \cdot \delta_{\mathrm{H}} \leq-L_{\mathrm{H}}-\frac{\Delta h_{\mathrm{SUP}}}{c_{\mathrm{air}}} .
\end{array}
$$

follow. The upper and lower bounds are

$$
\begin{aligned}
& L_{\mathrm{H}}=\dot{m}_{\mathrm{ODA}}^{\mathrm{ub}} \cdot\left(T_{\mathrm{ODA}}^{\mathrm{lb}}-T_{\mathrm{IDA}}^{\mathrm{ub}}\right)+\frac{\dot{Q}_{\mathrm{hp}}^{\mathrm{lb}}}{c_{\mathrm{air}}}-\frac{\Delta h_{\mathrm{SUP}}^{\mathrm{ub}}}{c_{\mathrm{air}}} \text { and } \\
& U_{\mathrm{H}}=\dot{m}_{\mathrm{ODA}}^{\mathrm{ub}} \cdot\left(T_{\mathrm{ODA}}^{\mathrm{ub}}-T_{\mathrm{IDA}}^{\mathrm{lb}}\right)+\frac{\dot{Q}_{\mathrm{hp}}^{\mathrm{ub}}}{c_{\mathrm{air}}}-\frac{\Delta h_{\mathrm{SUP}}^{\mathrm{lb}}}{c_{\mathrm{air}}} .
\end{aligned}
$$

Heating (el) If heating should only be done electrically, Equation (5.148) simplifies to

$$
\varphi_{\mathrm{ODA}} \cdot \dot{m}_{\mathrm{ODA}}^{\mathrm{ub}} \cdot\left(T_{\mathrm{ODA}}-T_{\mathrm{IDA}}\right)+\varphi_{\mathrm{eh}} \cdot \frac{\dot{Q}_{\mathrm{eh}}^{\mathrm{ub}}}{c_{\mathrm{air}}}=\frac{\Delta h_{\mathrm{SUP}}}{c_{\mathrm{air}}} .
$$


Consequently, the inequalities

$$
\begin{gathered}
\varphi_{\mathrm{ODA}} \cdot \dot{m}_{\mathrm{ODA}}^{\mathrm{ub}} \cdot\left(T_{\mathrm{ODA}}-T_{\mathrm{IDA}}\right)+\phi_{\mathrm{eh}} \cdot \frac{\dot{Q}_{\mathrm{eh}}^{\mathrm{ub}}}{c_{\mathrm{air}}}+U_{\mathrm{E}} \cdot \delta_{\mathrm{E}} \leq U_{\mathrm{E}}+\frac{\Delta h_{\mathrm{SUP}}}{c_{\mathrm{air}}} \text { and } \\
-\varphi_{\mathrm{ODA}} \cdot \dot{m}_{\mathrm{ODA}}^{\mathrm{ub}} \cdot\left(T_{\mathrm{ODA}}-T_{\mathrm{IDA}}\right)-\phi_{\mathrm{eh}} \cdot \frac{\dot{Q}_{\mathrm{eh}}^{\mathrm{ub}}}{c_{\mathrm{air}}}-L_{\mathrm{E}} \cdot \delta_{\mathrm{E}} \leq-L_{\mathrm{E}}-\frac{\Delta h_{\mathrm{SUP}}}{c_{\mathrm{air}}} .
\end{gathered}
$$

follow from that.

\subsubsection{HVAC model}

The HVAC model is used for calculating the currently applied manipulated variables, although a distinction is made between the two groups of modes. One contains heating and ventilation, the other cooling and dehumidification.

\section{Heating and ventilation}

For the modes of heating and ventilation, the matching energy balances (see Equation (5.111) respectively Equation (5.125)) easily transform to $\Delta h_{\text {SUP }}$

$$
\Delta h_{\mathrm{SUP}}=\varphi_{\mathrm{ODA}} \cdot \dot{m}_{\mathrm{ODA}}^{\mathrm{ub}} \cdot c_{\mathrm{air}} \cdot\left(T_{\mathrm{ODA}}-T_{\mathrm{IDA}}\right)+\phi_{H P} \cdot Q_{H P}^{\mathrm{ub}}+\phi_{H C} \cdot Q_{H C}^{\mathrm{ub}} \cdot
$$

The two other manipulated variables $\Delta Y_{\text {SUP }}$ and $\Delta \sigma_{\text {SUP }}$ follow from the transformation of the matching balance equation. Thus, Equation (1.25) allows to directly calculate $\Delta \sigma_{\text {SUP }}$ and for $\Delta Y_{\text {SUP }}$ follows analogously

$$
\Delta Y_{\mathrm{SUP}}=\dot{m}_{\mathrm{ODA}} \cdot\left(Y_{\mathrm{ODA}}-Y_{\mathrm{IDA}}\right) .
$$

This approach is possible because the heating and ventilation modes of the HVAC do not affect the absolute humidity. The balance equations of the manipulated variables can thus be solved independently of each other.

\section{Cooling and dehumidification}

In cooling and dehumidification, the energy balance (see Equation (5.120) respectively Equation (5.130)) cannot be transformed so easily. Since both manipulated variables $\Delta h_{\text {SUP }}$ and $\Delta Y_{\text {SUP }}$ appear together in one equation, another condition has to be found. This condition follows from the heat exchanger calculations in cooling [39]. For the heat exchanger of the CRM, the matching energy balance applies (cooling: Equation (5.120) and dehumidification: Equation (5.130)). The energy balance in cooling is

$$
\begin{array}{r}
\dot{m}_{\mathrm{ODA}} \cdot\left[c_{\mathrm{air}} \cdot\left(T_{\mathrm{ODA}}-T_{\mathrm{IDA}}\right)+\Delta h_{V o} \cdot\left(Y_{\mathrm{ODA}}-Y_{\mathrm{IDA}}\right)\right] \ldots \\
-\phi_{A C} \cdot Q_{A C}^{\mathrm{ub}}=\Delta h_{\mathrm{SUP}}+\Delta h_{V o} \cdot \Delta Y_{\mathrm{SUP}} .
\end{array}
$$


The terms of $\Delta h_{\text {SUP }}$ und $\Delta Y_{\text {SUP }}$ are substituted according to Equation (5.6) and Equation (5.7). After some transformations one gets

$$
\begin{gathered}
\alpha \cdot\left[c_{\mathrm{air}} \cdot\left(T_{\mathrm{ODA}}-T_{\mathrm{IDA}}\right)+\Delta h_{V o} \cdot\left(Y_{\mathrm{ODA}}-Y_{\mathrm{IDA}}\right)\right]+\Delta h_{V o} \cdot Y_{\mathrm{IDA}} \cdots \\
-\mathrm{COP}_{\mathrm{AC}} \cdot \frac{k_{r p m}}{\dot{m}_{\mathrm{SUP}}} \cdot \varphi_{\text {comp }}+c_{\mathrm{air}} \cdot T_{\mathrm{IDA}}=c_{\mathrm{air}} \cdot T_{\mathrm{SUP}}+\Delta h_{V o} \cdot Y_{\mathrm{SUP}}
\end{gathered}
$$

The mixing state of air before the heat exchanger follows from the outdoor and indoor air temperatures according to the equations

$$
\begin{aligned}
T_{\mathrm{MIA}} & =\frac{\dot{m}_{\mathrm{ODA}}}{\dot{m}_{\mathrm{SUP}}} \cdot T_{\mathrm{ODA}}+\frac{\dot{m}_{\mathrm{IDA}}}{\dot{m}_{\mathrm{SUP}}} \cdot T_{\mathrm{IDA}}, \\
Y_{\mathrm{MIA}} & =\frac{\dot{m}_{\mathrm{ODA}}}{\dot{m}_{\mathrm{SUP}}} \cdot Y_{\mathrm{ODA}}+\frac{\dot{m}_{\mathrm{IDA}}}{\dot{m}_{\mathrm{SUP}}} \cdot Y_{\mathrm{IDA}} .
\end{aligned}
$$

The temperature $T_{\mathrm{ac}}$ is the surface temperature of the heat exchanger. Suitable for this surface temperature, an absolute humidity $Y_{\mathrm{ac}}$ can be calculated since the air in the boundary layer is saturated $\varphi=1$. The states at the exit of the heat exchanger follow from the law of the lever.

$$
\begin{aligned}
& \left(T_{\mathrm{MIA}}-T_{\mathrm{ac}}\right) \cdot \beta+T_{\mathrm{AAC}}=T_{\mathrm{MIA}}, \\
& \left(Y_{\mathrm{MIA}}-Y_{\mathrm{ac}}\right) \cdot \beta+Y_{\mathrm{AAC}}=Y_{\mathrm{MIA}} .
\end{aligned}
$$

The electric heater reheats the air leaving the heat exchanger in the dehumidification mode. So the states of the supply air can be calculated

$$
\begin{aligned}
T_{\mathrm{SUP}}-T_{\mathrm{AAC}} & =\frac{k_{H C}}{c_{\mathrm{air}} \cdot \dot{m}_{\mathrm{SUP}}} \cdot \varphi_{H C}, \\
Y_{\mathrm{SUP}}-Y_{\mathrm{AAC}} & =0 .
\end{aligned}
$$

The listed equations of Equation (5.162), Equation (5.165), Equation (5.166), Equation (5.167) and Equation (5.168) represent a linear system of equations of the form

$$
\mathbf{A} x=\mathbf{b}
$$

From the solution of the linear system, $T_{\text {SUP }}$ and $Y_{\text {SUP }}$ follow directly so that $\Delta h_{\text {SUP }}$ and $\Delta Y_{\text {SUP }}$ can be calculated. Since Equation (5.169) must often be solved during a simulation run, it is more efficient to analytically calculate $\mathbf{A}^{-1}$ only once.

\subsubsection{Solution approach}

The optimisation problems presented and described in Section 5.4.2 and Section 5.4.3 need to be solved by a proper method. For that purpose, three different solution approaches are discussed

- Complete numbering

- Exact solution by an external solver

- Heuristic

In addition, the pros and cons of the individual methods are discussed. 


\begin{tabular}{crrrrrr} 
Table 5.1. - Number of solutions of the MILP (Complete numberin \\
\hline- & $\mathscr{H}$ & $\mathscr{E}$ & $\mathscr{B}$ & $\mathscr{V}$ & $\mathscr{C}$ & $\mathscr{D}$ \\
\hline$\dot{m}_{\text {ODA }}$ & 20 & 20 & 20 & 20 & 20 & 20 \\
$\dot{m}_{\text {IDA }}$ & 21 & 21 & 21 & 21 & 1 & 1 \\
$\varphi_{\text {comp }}$ & 13 & 1 & 1 & 1 & 13 & 1 \\
$\varphi_{H C}$ & 1 & 100 & 100 & 1 & 1 & 100 \\
$\Pi$ & 5460 & 42000 & 42000 & 420 & 260 & 2000 \\
\hline
\end{tabular}

\section{Complete numbering}

As the manipulated variables of the HVAC are discretized, the complete numbering of the solution space allows finding the solution. It finds the best possible solution because this method calculates all possible solutions, but needs much computational effort. This calculation takes about one minute on a current PC (Intel Core i7 $860 @ 2.80 \mathrm{GHz}$ ). For the controller, with a sampling time of $10 \mathrm{~s}$, this method is not applicable. However, for a preliminary study and a first proof of the equations this method can be used, if calculating all solutions needs a reasonable time. Table 5.1 shows a more detailed approach that analyses the number of possible solutions, separately for each mode.

\section{External solver}

Equation (5.51) gives the (mixed) integer optimisation problem. Section 5.4.2 and Section 5.4.3 introduce the description of equations and inequalities. Now the problem can be solved by using a proper solver. As Matlab has no own solver for that problem class, an external solver called lpsolve [11] was used. lpsolve is a free solver for mixed integer problems. It is in $\mathrm{C} / \mathrm{C}++$, has many different interfaces (also including Matlab) and, depending on the given problem, the solver finds a solution in about $100 \mathrm{~ms}$ to $1 \mathrm{~s}$.

However, debugging mixed integer problems is difficult and complex. Already typing errors might lead to a not solvable problem. Every inequality has to be evaluated manually by inserting a known solution (calculated by complete numbering, for example). Another disadvantage of this approach is that the solver is provided as a shared library which cannot be used on the RCPP. Instead of dynamic linking, static linking would also be possible, but that will cause juristic problems with the software license. As consequence, the approach was not pursued.

\section{Heuristic}

The heuristic solves the (mixed) integer linear problem. The problem is split into two parts, which reduces the problem size.

- Estimating the HVAC mode

- Calculating the control variables of the HVAC

Estimating the HVAC mode The following modes exist for the air conditioning - $\mathscr{H}$ - Heating (CRM), 


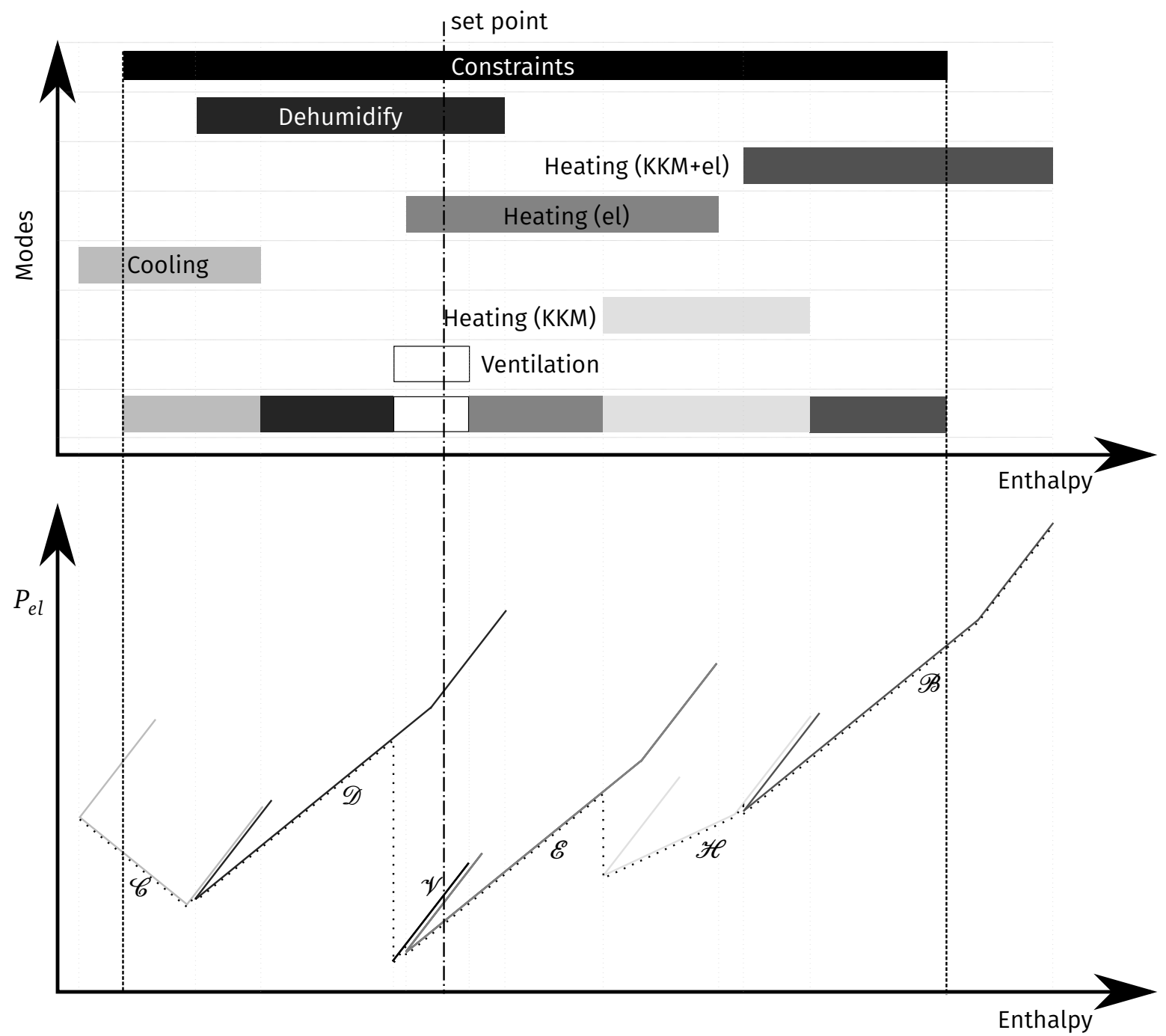

Figure 5.6. - Both methods for estimating the HVAC mode

- $\mathscr{E}$ - Heating (el),

- $\mathscr{B}$ - Heating (CRM+el),

$-\mathscr{V}$ - Ventilation,

- $\mathscr{C}$ - Cooling and

- $\mathscr{D}$ - Dehumidification.

Two different approaches were tested for estimating the HVAC mode.

- Prioritising modes by expert knowledge

- 2D constraints

The following section presents both approaches and discusses their pros and cons.

Prioritising modes by expert knowledge The basic idea of this method is that one can assign a fixed priority to the individual modes. This assumes that it is always more energy efficient if 
ventilation is used instead of Heating (el), for example.

For that purpose, the upper and lower boundaries of the supplied energy $\Delta h_{\text {SUP }}$ are calculated for each mode. As the boundaries can overlap, ranking is used to achieve a unique assignment. The sequence is (beginning from the best mode):

1. $\mathscr{V}$ - Ventilation

The most energy conserving mode because only the fan consumes electrical power.

2. $\mathscr{H}$ - Heating (CRM)

If heating is needed, this will be the most efficient way.

3. $\mathscr{C}$ - Cooling

In general, the COP during cooling is smaller than during heating.

4. $\mathscr{E}$ - Heating (el)

The original version of heating has a COP of (almost) 1 .

5. $\mathscr{B}$ - Heating $(\mathrm{CRM}+\mathrm{el})$ If needed, heating can be done electrically and by the CRM at the same time.

6. $\mathscr{D}$ - Dehumidification

The unfavourable case, in which both cooling and electric heating is on.

In addition, the optimisation considers constraints on the supply air temperature. Preferring the mode with a lower number removes the overlaps. For a given set point $\Delta h_{\mathrm{SUP}}$ a unique mode can be determined now. Figure 5.6 (above) graphically shows this calculation. A thick beam shows the feasible areas of the modes. They are drawn from the best mode (Ventilation) at the bottom to the worst mode (Dehumidification) at the top. The unique assignment is shown below the modes, the constraint for the supply air temperature above. The advantage is, that it promotes an easy and intuitive calculation and achieves a fast execution time. The disadvantage is, that the current energy consumption is not calculated. The assumption about the energy consumption may be wrong, which is especially true for the boundary area of the modes.

2D constraints Acting on the disadvantages of the first method, a second method was developed. One can see it in Figure 5.6 (below). The P-h diagram depicts the different modes as areas. Only the edges with the least power consumption of these areas are interesting. So the minimum of all edges is sought. Figure 5.6 (below) shows the minimum as a black dotted line. The following paragraph briefly explains the calculation. The four vertices of each mode are calculated

- minimum supplied enthalpy and minimum outdoor airflow $\mathscr{P}_{1}\left(h^{\mathrm{lb}}, \dot{m}_{\mathrm{ODA}}^{\mathrm{lb}}\right)$

- minimum supplied enthalpy and maximum outdoor airflow $\mathscr{P}_{2}\left(h^{\mathrm{lb}}, \dot{m}_{\mathrm{ODA}}^{\mathrm{ub}}\right)$

- maximum supplied enthalpy and minimum outdoor airflow $\mathscr{P}_{3}\left(h^{\mathrm{ub}}, \dot{m}_{\mathrm{ODA}}^{\mathrm{ub}}\right)$

- maximum supplied enthalpy and maximum outdoor airflow $\mathscr{P}_{4}\left(h^{\mathrm{ub}}, \dot{m}_{\mathrm{ODA}}^{\mathrm{lb}}\right)$

With the minimum supplied enthalpy, for example, the enthalpy by the minimum compressor power during cooling is meant here. The calculated vertices may not satisfy the constraints. In the ventilation mode both points $\mathscr{P}_{1}$ and $\mathscr{P}_{4}$ respectively $\mathscr{P}_{2}$ and $\mathscr{P}_{3}$ coincide with each other. A square results in every other mode, when the points are connected by lines. The lines are $\overline{\mathscr{P}_{2} \mathscr{P}_{1}}$, $\overline{\mathscr{P}_{1} \mathscr{P}_{4}}$ and $\overline{\mathscr{P}_{4} \mathscr{P}_{3}}$. The distance $\overline{\mathscr{P}_{3} \mathscr{P}_{2}}$ may be omitted. 
Calculating the manipulated variables of the HVAC This paragraph describes the heuristic which converts the set point of the master controller into the discrete switching modes of the HVAC $x_{\text {MILP }}$. Depending on the chosen mode, a different algorithm is used, in which the basic idea remains the same.

It is always iterated over the outdoor airflow and, if it can be changed, over the recirculation airflow. The conditions of the supply air are estimated for the given supply airflow. The remaining manipulated variable (depending on the mode) can usually be calculated now. One gets the wanted switching conditions $x_{\text {MILP }}$ by rounding up or down. The energy consumption, the supply air conditions and the currently supplied enthalpy are calculated for all of these $i$ switching possibilities. Calculating a switching condition is stopped as early as possible, to waste as little as possible computation time with infeasible solutions. A switching possibility is infeasible if the constraint of the supply air temperature is violated, for example. From all valid solutions, the solution with the lowest possible energy consumption $P_{\text {eh }}$ and a small difference to the set point is selected. This happens by solving the unrestricted optimisation problem

$$
\min _{i} J=P_{\mathrm{eh}}(i)+\left(h_{\mathrm{MILP}}(i)-h_{\mathrm{MPC}}\right)^{2}
$$

in $i$, in which $i$ is the index of the switching possibilities calculated before.

The following paragraphs explain the individual parts of the heuristic in detail. They discuss the characteristics of each heuristic part and explain the heuristic further by pseudo-code.

Heuristic for the cooling mode In the cooling mode, the CRM works in cooling and the compressor is switched on. Due to the constraints of the HVAC, the supply air only consists of outdoor air. The remaining control variables are the outdoor airflow and the level of the compressor.

Algorithm 1 shows the pseudo-code for the cooling heuristic; some changes are made to the default template. As there are only a few possibilities in the cooling mode (see Table 5.1), the algorithm calculates all possibilities and chooses the most fitting one.

Dehumidification In the dehumidification mode the CRM works in cooling and the compressor is switched on. The smallest compressor rotational speed is selected definitely. Due to the constraints of the HVAC, the supply air must only consist of outdoor air, recirculation air is not possible. The remaining manipulated variables are the outdoor airflow and the levels of the electric heater.

Ventilation In the ventilation mode the compressor is switched off. Due to the constraints of the HVAC, the supply air must only consist of outdoor air and the electric heater must be turned off. The only remaining control variable is the outdoor airflow.

Heating (CRM) In the heating (CRM) mode the CRM works in heating mode and the compressor is switched on. The electric heater is turned off. The supply air can be mixed from the recirculation air and the outdoor air. So one gets three control variables: outdoor airflow, recirculation airflow and compressor level.

Heating (el) In the heating (el) mode the CRM is switched off. Heat is only supplied by the electric heater. Recirculation air and outdoor air mix to supply air. So three control variables follow: 


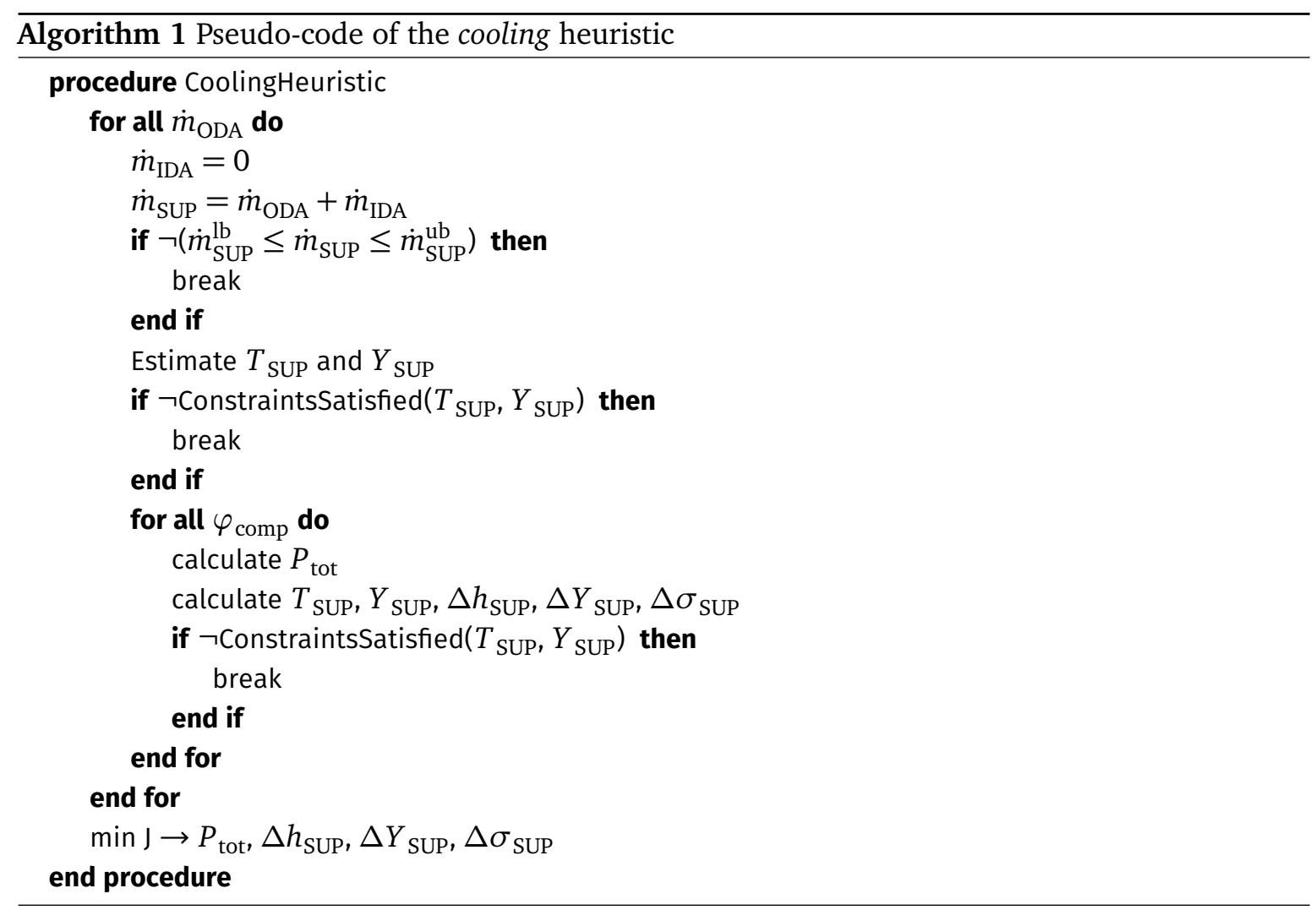

outdoor airflow, recirculation airflow and the level of the electric heater.

Heating (el+CRM) In the heating (el+CRM) mode the CRM works with the highest compressor level. The electric heater is turned on. The supply air may consist of recirculation air and outdoor air. So the control variables follow: outdoor airflow, recirculation airflow and the level of the electric heater.

\section{Improvement heuristic}

This improvement heuristic was developed based on a suggestion from our project partners to prevent the use of the dehumidification and heating (el) modes. To achieve the previously defined objective of the optimisation problem (at first an optimal comfort and then a low energy consumption), this method need not be used. The performed simulation suggests that the method is rarely used. The sought solutions are left below the targeted solution in Figure 5.2. It is not guaranteed, whether this solutions also lies on the Pareto-front.

The basic idea of this improvement heuristic is preventing the dehumidification and heating (el) mode by switching to neighbouring modes. In doing so, various constraints have to be considered. A conventional approach for this behaviour can be achieved using a 2-point controller with hysteresis, this work uses a method based on the idea of the MPC (see Section 2.3.3). 
Objective Figure 5.6 shows that there are two possibilities for preventing the dehumidification mode, either by choosing the cooling mode or the ventilation mode. In both cases, the thermal comfort worsens because the controller supplies either too much or too little enthalpy. The described relation analogously applies to the mode heating (el), the neighbouring modes are ventilation and heating $(\mathrm{kkm})$. A sequence of the control variable is to be found over the horizon $T$ which satisfies the thermal comfort constraints. There are only two possibilities for the control variable $u$, that is $u \in\left[u_{1}, u_{2}\right]$.

Optimisation problem The optimisation problem is also an MPC problem

$$
\begin{aligned}
\min & \sum_{t=1}^{T} \iota \cdot\left(x^{\text {set }}(t)-x(t)\right)^{2} \\
\text { subject to } & x(t+1)=\mathbf{A}(t) x(t)+\mathbf{B}(t) u(t)+Z(t) \\
& x^{\mathrm{lb}}(t) \leq x(t) \leq x^{\mathrm{ub}}(t), \quad u \in\left[u_{1}, u_{2}\right] .
\end{aligned}
$$

\subsection{Implementation}

This subsection describes the implementation of the controller. Based on an executable Simulink version, the algorithms are ported to the chosen RCPP. This section explains the used hardware and then discusses the used software for this hardware.

\subsubsection{Hardware}

The original plan was that Vossloh Kiepe (VK) set up the developed controller algorithms on the HVAC controller. Already the first estimations of the needed execution time showed that this approach would not succeed. The Vienna University of Technology (VUT) therefore bought an RCPP to set up the algorithms. The controller platform allows directly connecting all extra needed sensors. A proper hardware has to be chosen and a protocol must be implemented for the data exchange between the HVAC and the controller.

\section{Controller platform}

The product MicroAutobox II from dSpace was bought as RCPP because of its good link to Matlab and Simulink. The algorithms developed in Matlab can be ported with a maintainable effort to the controller platform. The datasheet [26] lists the technical specification of the platform.

\section{Sensors}

Most of the needed sensors are directly connected to the HVAC controller. However, there was no interface left for

- the sun sensor [104] and

- the Global Positioning System (GPS) receiver [40]. 


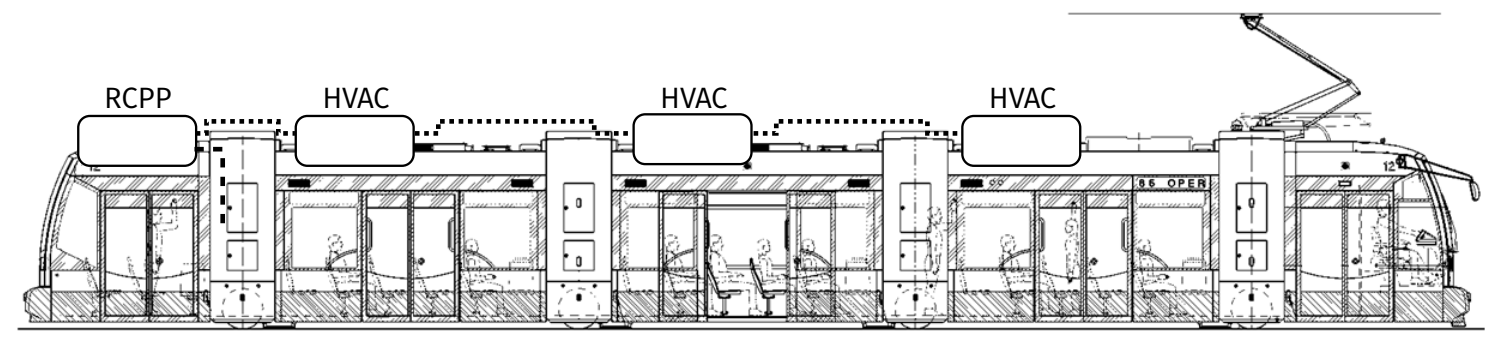

Figure 5.7. - Composition of the RCPP and the HVAC controllers on the tram. The figure shows the Controller Area Network (CAN) cable between the RCPP and the HVAC controller as a black dotted line and the power supply of the RCPP as a dashed line.

The sun sensor measures the angels and the intensity of the incident sunlight and the GPS receiver is needed for the disturbance rejection.

\section{Communication}

A bus system called CAN was used for the communication between the HVAC and the controller. The first HVAC works as the bus master. Figure 5.7 shows the composition used. A connection to the bus of the tram (Multi-function Vehicle Bus (MVB)) is possible but was not done due to safety reasons.

\subsubsection{Software}

The advantage of the MicroAutobox II is the extensive support of Simulink and therefore also of embedded Matlab. Embedded Matlab is a small subset of all Matlab functions, which are available in Simulink. It does not include both solvers for linear and quadratic problems linprog respectively quadprog. [107] contains a list of the available functions. The following paragraphs describe setting up the controllers, sensors and the bus communication.

\section{Controller}

The above-mentioned restrictions require to use the solution approach described in Section 5.3. Based on the work of Wang and Boyd [119], the C implementation was extended, as described in Section 5.3. After specifying the C function for the MPC in Simulink, embedded Matlab code allows calling the function. The used libraries blas and lapack [3] were compiled using the dSpace toolchain (compiler and linker) and were also specified in Simulink. The slave controller was implemented in Matlab and was directly called from Simulink as embedded code. A C implementation would have been possible but was not necessary because of the fast execution time. 


\section{Sensors}

The solar radiation sensor requires to develop a special communication protocol based on the specifications of the manufacturer. The RCPP needs to send a command by a serial link to the sensor. The following answer contains the measurements. A simple parsing state machine allows parsing the reply string to get the measurement. A standardised protocol called NMEA-0183 [4] exists for communicating with the GPS receiver, which is supported by the used GPS receiver [40]. dSpace has already set up the protocol as an S-function, which is provided as free download [27]. So, this implementation is used. 



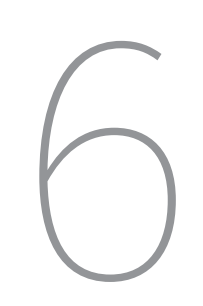

\section{Results}

This chapter summarises all the results of this thesis. The first section shows the results of the parameter estimation (see Chapter 4). Then it describes validating the estimated parameters for the developed models (see Chapter 3), using the first climatic wind tunnel measurements and the first on-site measurements. The next subsection shows simulation results of the controller design (see Chapter 5) individually for the master and slave controller. The controller runs on the RCPP and the RCPP is integrated into the tram. The achieved results are shown with the help of representative experiments for the second climatic wind tunnel measurements. These experiments compare energy consumptions of the first and the second climatic wind tunnel measurements. In the end, the chapter compares the calculated annual energy consumption of the current and the new tram.

\subsection{Model Validation}

The described model of the vehicle and the HVAC (see Chapter 3) must be validated. Both the validation results obtained using climatic wind tunnel measurements and the validation results from the on-site measurements are shown and discussed.

Hofstädter and Kozek [51] had already published the results shown in this section. The authors of [97] provided measurement data from climatic wind tunnel experiments, sampling time was $10 \mathrm{~s}$.

\subsubsection{Validation using Climatic Wind Tunnel Measurement Data}

Environmental conditions varied as follows

1. Outdoor air temperature: $-20^{\circ} \mathrm{C}$ to $35^{\circ} \mathrm{C}$

2. Passenger simulation: 0 to 136 persons (maximum capacity) and

3. Sun radiation: from $0 \mathrm{~W} / \mathrm{m}^{2}$ to $800 \mathrm{~W} / \mathrm{m}^{2}$.

[95] shows a more detailed summary of the conducted experiments. Due to the vertical layering of air in the tram, the air temperature range is quite large. Nevertheless, the simulation results stay between maximum (Max) and minimum (Min) measurements for stable environmental conditions 

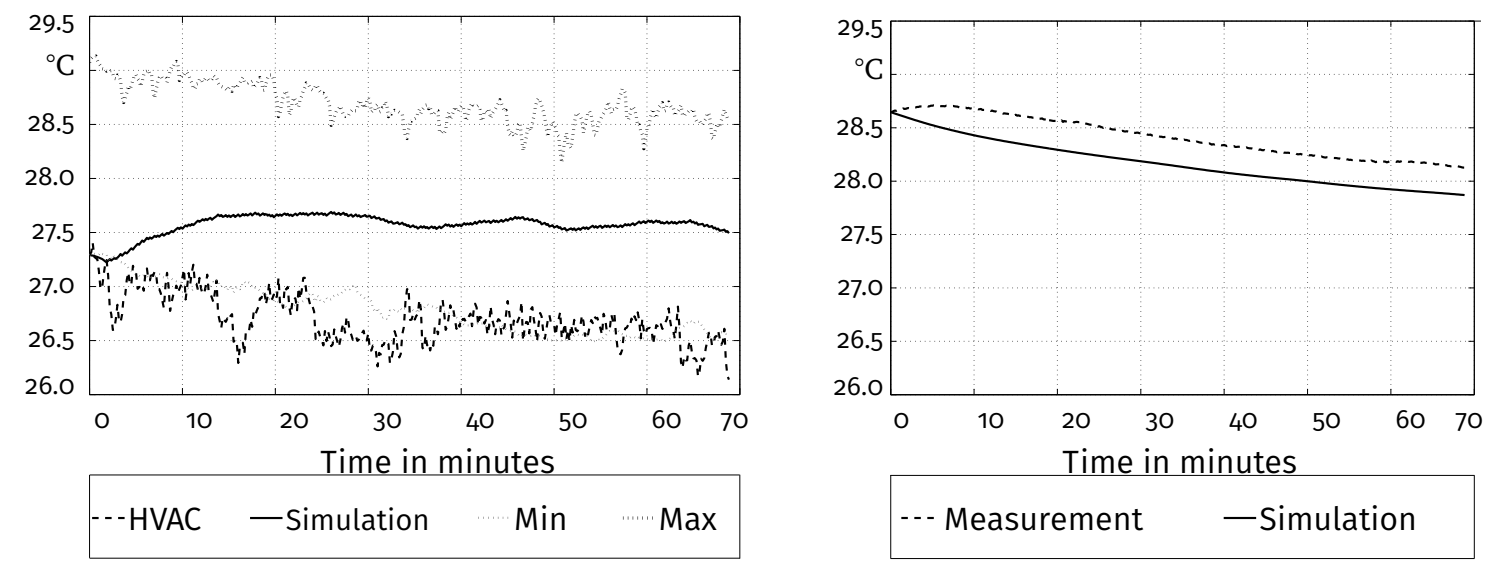

Figure 6.1. - Section 2 - left: indoor air temperature, right: temperature of car body Environmental conditions: $T_{\mathrm{ODA}}=28^{\circ} \mathrm{C}$, sun simulation: $800 \mathrm{~W} / \mathrm{m}^{2}$, passenger simulation: o persons

Simulation results stay between minimum and maximum measurements. The deviation is small for the temperature of the car body.

as seen in Figure 6.1. Even in dynamic conditions, the simulation deviation is small, considering the long simulation time (see Figure 6.2). The air conditioning model is accurate in both heating and cooling. Since cooling is harder to simulate, Figure 6.3 shows a simulation result of a $28^{\circ} \mathrm{C}$ cooling experiment.

The tram model can calculate the indoor air temperature quite accurately for most working points, and the HVAC model provides satisfying results in stationary working points.

\subsubsection{Validation using On-Site Measurement Data}

Validating models by on-site measurement data was mainly affected by the merging of several measurement data from different sources. This was necessary to study miscellaneous influences and their effects on the temperatures inside the tram.

For that purpose, an automatic evaluation of the measurement data was done, which explains all available input variables for the trams. Running the simulation needs these data. Annex B (see Appendix B.1) shows an example of those simulation reports. They provide the following information:

- Environmental conditions (weather, a map of the travelled route, distance-time graph, outdoor air temperature, solar radiation on to the tram, number of passengers (load))

- Tram (measurement and simulation of the indoor air temperature, the relative humidity and the thermal comfort stated as the PMV and the PPD)

- HVAC (Temperature and humidity of the supply air, energy consumption of $400 \mathrm{~V}$ and $600 \mathrm{~V}$ consumers as well as several comparisons of the measured and simulated consumption)

- Controller (global state of the controller)

The simulation model usually turns out to be acceptably robust against occurring unknown disturbances. 

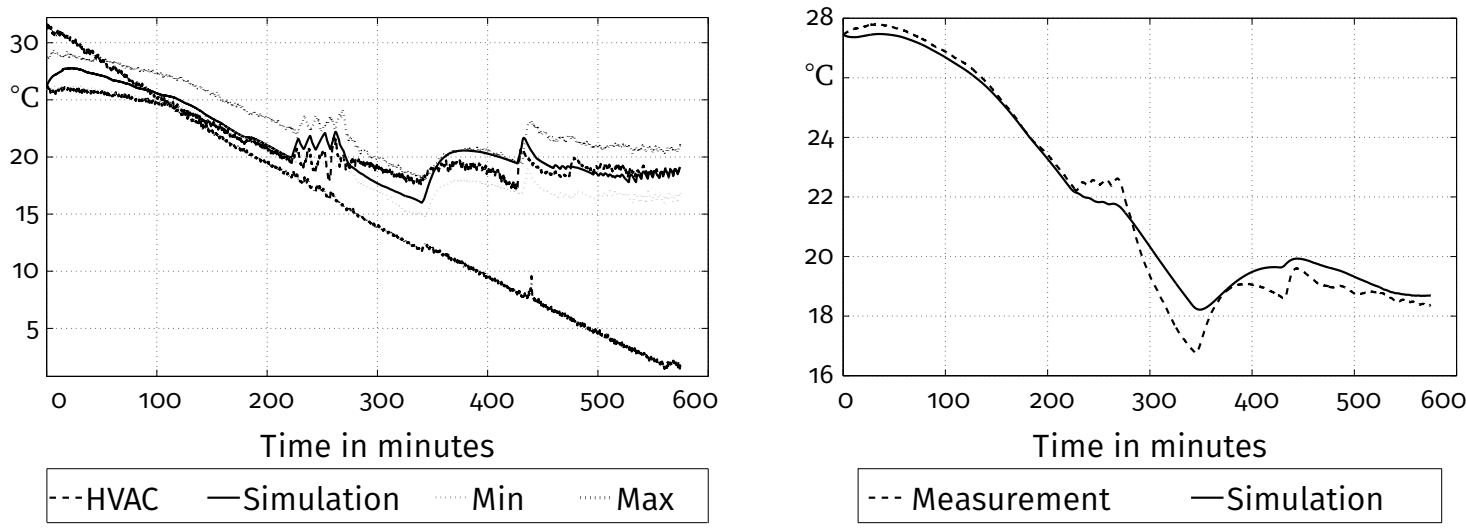

Figure 6.2. - Section 2 - left: indoor air temperature, right: temperature of car body Environmental conditions: $\mathrm{T}_{\mathrm{ODA}}: 0^{\circ} \mathrm{C}$ to $28^{\circ} \mathrm{C}$, sun simulation: $600 \mathrm{~W} / \mathrm{m}^{2}$, passenger simulation: 43 persons

Simulation results stay between minimum and maximum measurements. The deviation is small for the temperature of the car body.

\subsection{Grey-box modelling}

Hofstädter et al. [56] had already published the results described in this section. In fact, this section is a literal quote of the "results" chapter of this paper. The text was only slightly adapted in the choice of words and some grammar and spelling mistakes were corrected.

First, this section compares the first-order and the second-order model. Then it discusses results for the estimated parameters $\left(C, k, C_{v e h}, K_{v e h}\right)$ and generalises the estimated parameters for individual vehicles for different vehicle classes.

\subsubsection{Model order}

A first-order and a second-order grey box model are estimated for the tram Tram2 using special experiments. Time series plots compare measurement data to simulation results in with Figure 6.4. Both models can satisfactorily reproduce the measurement data. Since second-order models are almost only used in literature and provide a slightly better model fit, they are used for all trains.

Estimating a black box model would achieve an even better fit to measurement data. But this approach loses the analytical structure of the model. A generalisation of the model is no longer possible, and no conclusion could be drawn for similar trains. Thus, the second-order model used for all trains is a grey box model.

\subsubsection{Estimated parameters}

Measurement data from the CWT were sighted, preprocessed and input into the developed software for the existing experiments. Table 4.1 show an outline about the conducted and evaluated experiments. Many experiments had to be discarded because measurement data did not meet the underlying assumptions (see Section 4.1.4). 

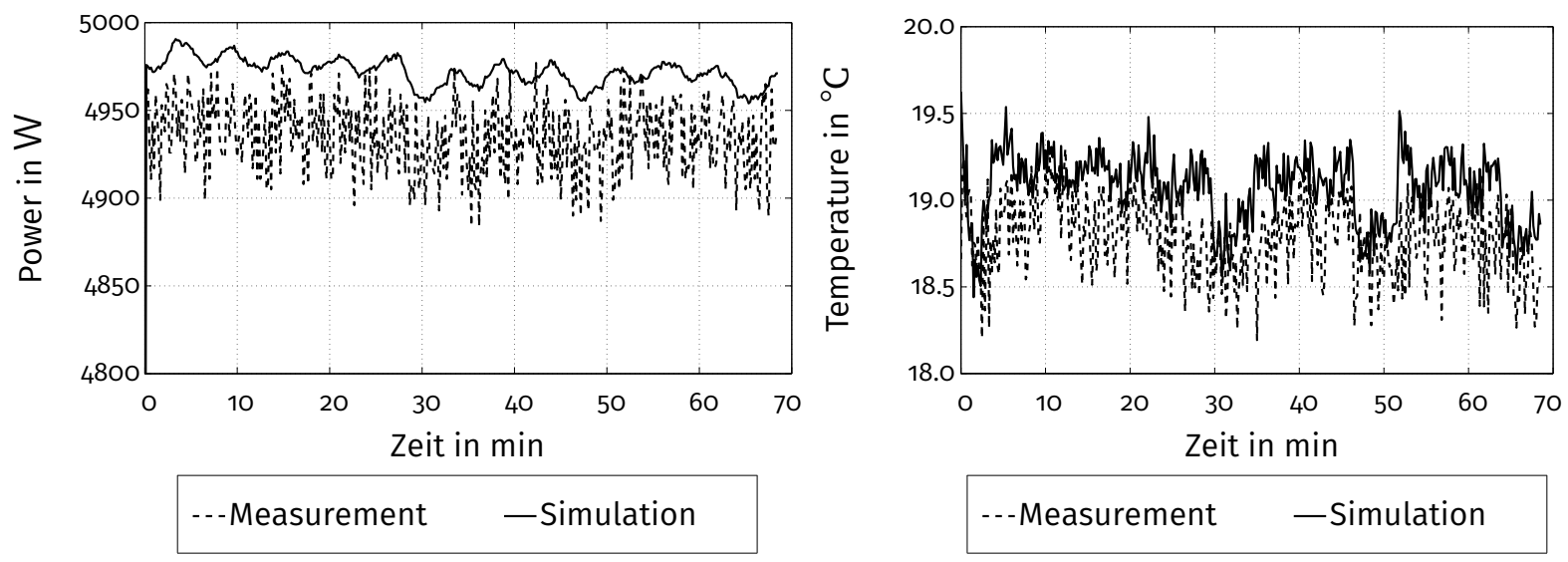

Figure 6.3. - left: power consumption of compressor and supply air fan; right: supply air temperature Environmental conditions: $T_{\mathrm{ODA}}: 28{ }^{\circ} \mathrm{C}$, sun simulation: $800 \mathrm{~W} / \mathrm{m}^{2}$, passenger simulation: o persons - deviation of power consumption is about $1 \%$, deviation of supply air temperature in measurement accuracy band

Table 4.2 shows a summary of average values for all estimated parameters of all identified trains.

Using the estimated parameters, the simulation model was established. Figure 6.5 shows a comparison between simulation and measurement of the indoor air temperature for two metros. Parameters for a model with small deviations could be estimated for all trains, as both examples show. The deviation between measurement and simulation is similarly small as for Tram2, but the considered temperature range is smaller.

\subsubsection{Generalized parameters}

Besides absolute results, the specific heat capacity $c_{v e h}$ of the car body is calculated by

$$
c_{v e h}=\frac{C_{v e h}}{m_{v e h}}
$$

where $m_{v e h}$ is the mass of the train.

Results are grouped by train type and plotted in Figure 6.6a. The figure uses the train type because features of a train correlate usually well with the train type. So the assumption is that various features of the train, such as number of seats, type of seats (with a fabric cover or plastic only) and interior decoration can be mapped into the train class. For example, metros and trams mostly contain seats made of plastic, while mainline trains have more comfortable seats with fabric cover. For the two classes, tram and mainline, no further conclusion is possible because of the limited data set. Figure 1.3a shows that two classes, metros and regional trains, differ from each other. The mean value of the specific heat capacity for metros is $372 \mathrm{~J} /(\mathrm{kg} \mathrm{K})$, and for regional trains it is $603 \mathrm{~J} /(\mathrm{kg} \mathrm{K})$. Due to the small numbers of measurements and the high variance inside the train classes, it cannot be statistically proven that the difference is significant. Another 


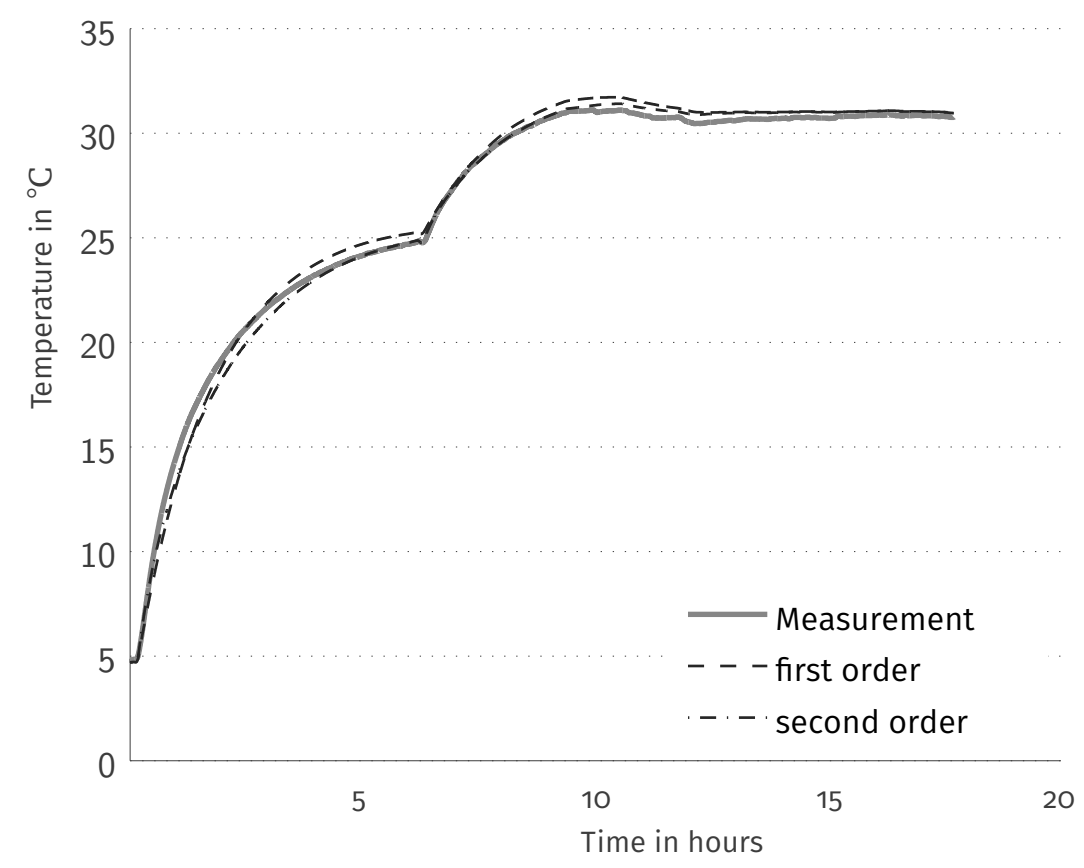

Figure 6.4. - Tram2: Comparison between different models (Simulation) and measurement using data from step responses

generalised result is the ratio $\delta$ of the two heat capacities, which can be calculated by

$$
\delta=\frac{C_{v e h}}{C} .
$$

Figure $6.6 \mathrm{~b}$ shows the ratio for different train classes. Table 6.1 lists the matching numbers. The ratio increases from tram through metro and regional to mainline. Just as expected since mainline trains are a lot heavier than trams and metros. So their car body has a larger heat capacity.

\subsubsection{Plausibility check}

Since only a small amount of data is available, a cross-validation of models is not possible. Instead, a plausibility check of results is done. There is a maximum of one usable experiment for each train and this experiment is used for the parameter estimation. Table 6.1 and Figure 6.6a show that values for the specific heat capacity for the train $c_{v e h}$ range from $175 \mathrm{~J} /(\mathrm{kg} \mathrm{K})$ to $703 \mathrm{~J} /(\mathrm{kg} \mathrm{K})$. Values are exactly in the expected range. The train especially needs steel $(c \approx 477 \mathrm{~J} /(\mathrm{kg} \mathrm{K})$ ) and aluminium $(c \approx 896 \mathrm{~J} /(\mathrm{kg} \mathrm{K}))$ in larger quantities during building.

\subsubsection{Conclusion}

This section presents the estimation of heat capacities and heat transfer coefficients for two dynamic thermal rail train models. It uses different sources of measurement data. While special 


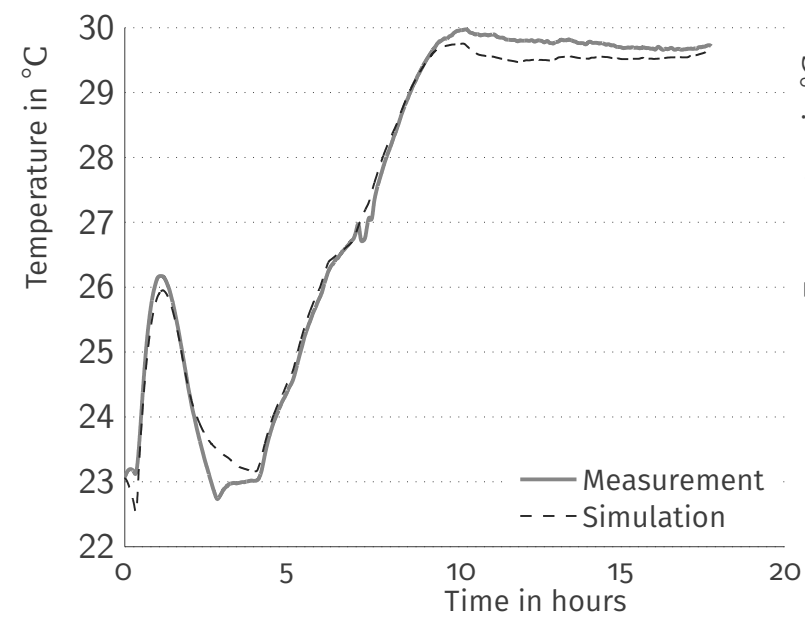

(a) Metro1

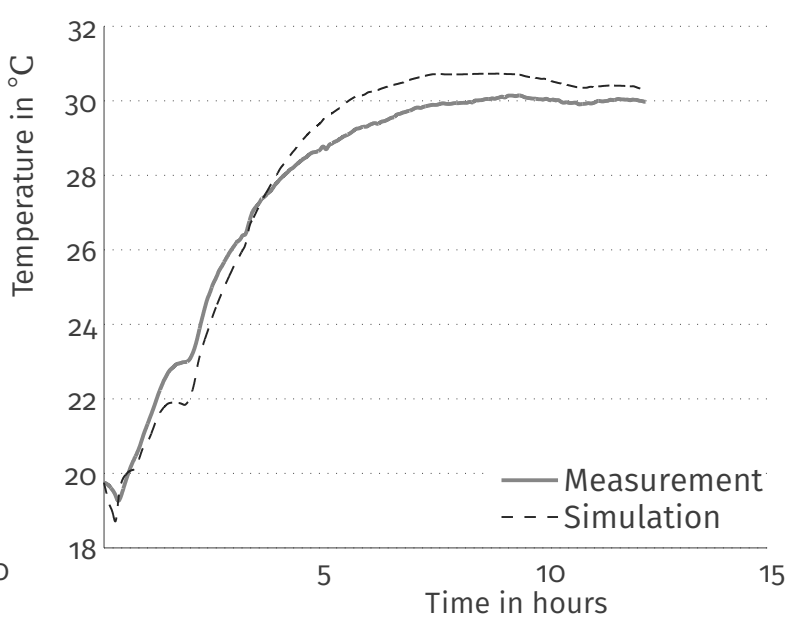

(b) Metro5

Figure 6.5. - Comparison between estimated models (simulation) and measurements for indoor air temperature using data from standard commissioning

experiments are designed for the estimation, some existing measurements can sometimes be used, too, and comparable results are obtained. Simulation results are in good agreement with measurement data if underlying model assumptions are met. This section provides parameters for all relevant passenger train classes: Tram, Metro, Regional and Mainline. It also shows that grouping into these train classes is justified. The plausibility check shows that the gained results are in the expected range and correlate with the train construction. The estimation results and the generalised parameters provide the first step towards a basis for future thermal train models for all relevant train classes.

This provides a basis for a wide application in the industry: The train manufacturer can fall back on the proposed parameters for the dynamic energy consumption calculation (which is already done occasionally) until those parameters have been measured in the climatic wind tunnel.

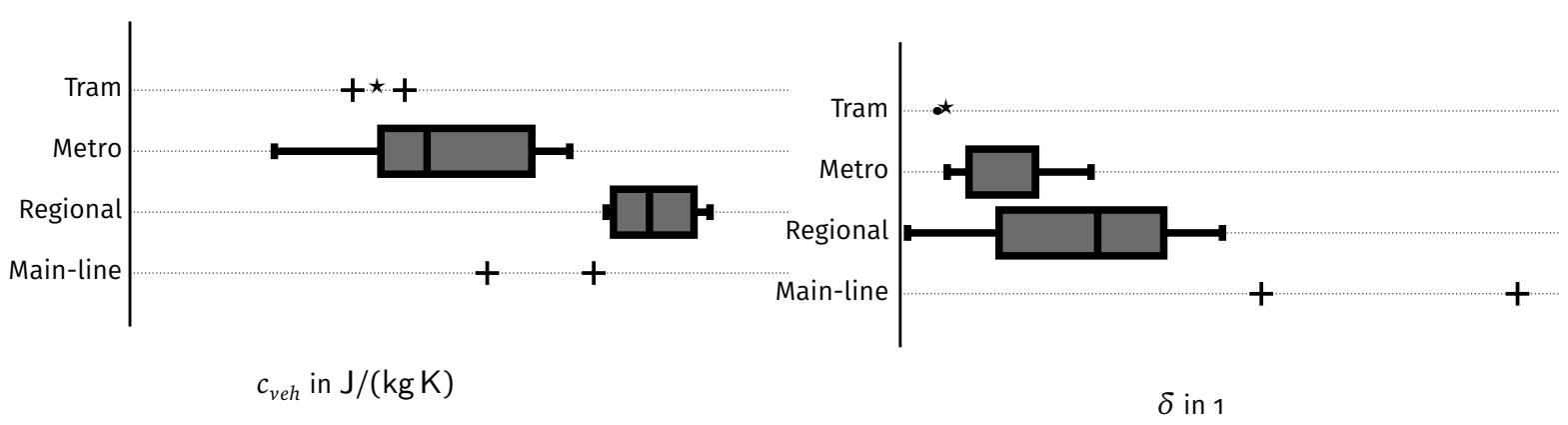
$\begin{aligned} & \text { (a) Specific heat capacity of the vehicle body } c_{v e h} \text { versus } \\ & \text { vehicle class }\end{aligned}$
(b) Ratio of the two heat capacities $\delta$ versus vehicle class

Figure 6.6. - Boxplots of generalised parameters for each vehicle class 


\begin{tabular}{|c|c|c|}
\hline Name & $\begin{array}{c}c_{v e h} \\
\mathrm{~J} /(\mathrm{kgK})\end{array}$ & $\begin{array}{l}\delta \\
1\end{array}$ \\
\hline Tram1 & 270 & 0.56 \\
\hline Tram2 & 333 & 0.55 \\
\hline Metro1 & 533 & 2.05 \\
\hline Metro2 & 304 & 1.04 \\
\hline Metro3 & 175 & 0.71 \\
\hline Metro4 & 487 & 2.89 \\
\hline Metro5 & 360 & 2.05 \\
\hline Regio1 & 664 & 0.11 \\
\hline Regio2 & 577 & 4.88 \\
\hline Regio3 & 595 & 3.1 \\
\hline Regio4 & 703 & 2.88 \\
\hline Main1 & 562 & 5.47 \\
\hline Main2 & 433 & 9.35 \\
\hline
\end{tabular}

Calculations will be easier to understand for the customers and operators of trains if they use consistent parameters as a standard for the thermal train model. With the proposed parameters, numerical values for the dynamic thermal train models are available to the manufacturer during the design phase of the HVAC. In addition, the proposed dynamic thermal train models are the basis for further optimisation of all thermal parts of the train. Thus, a modern controller (that is a model-based predictive controller) can be designed which uses the proposed models for energy consumption optimisation. The proposed parameters support the designer because a laborious analytical calculation is not needed. Measurements of heat capacity and heat transfer on all future trains would be favourable.

\subsection{Simulation results of the controller}

The simulation results presented in this section of the controller are common result. Changes in one part of the controller have almost always an effect on the other part.

The first subsection shows the simulation results of the master controller. The control objective is maximising the thermal comfort, in the specific case a small deviation of the current temperature from the set point temperature, in which various constraints have to be considered. The slave controller converts the set point of the master controller in an energy-optimal way. This section shows two approaches: Based on a full enumeration of all possibilities, the entire solution set is pictured. The diagram of the heuristic shows that one needs to search only a small part of the solution set to find an equal solution. 


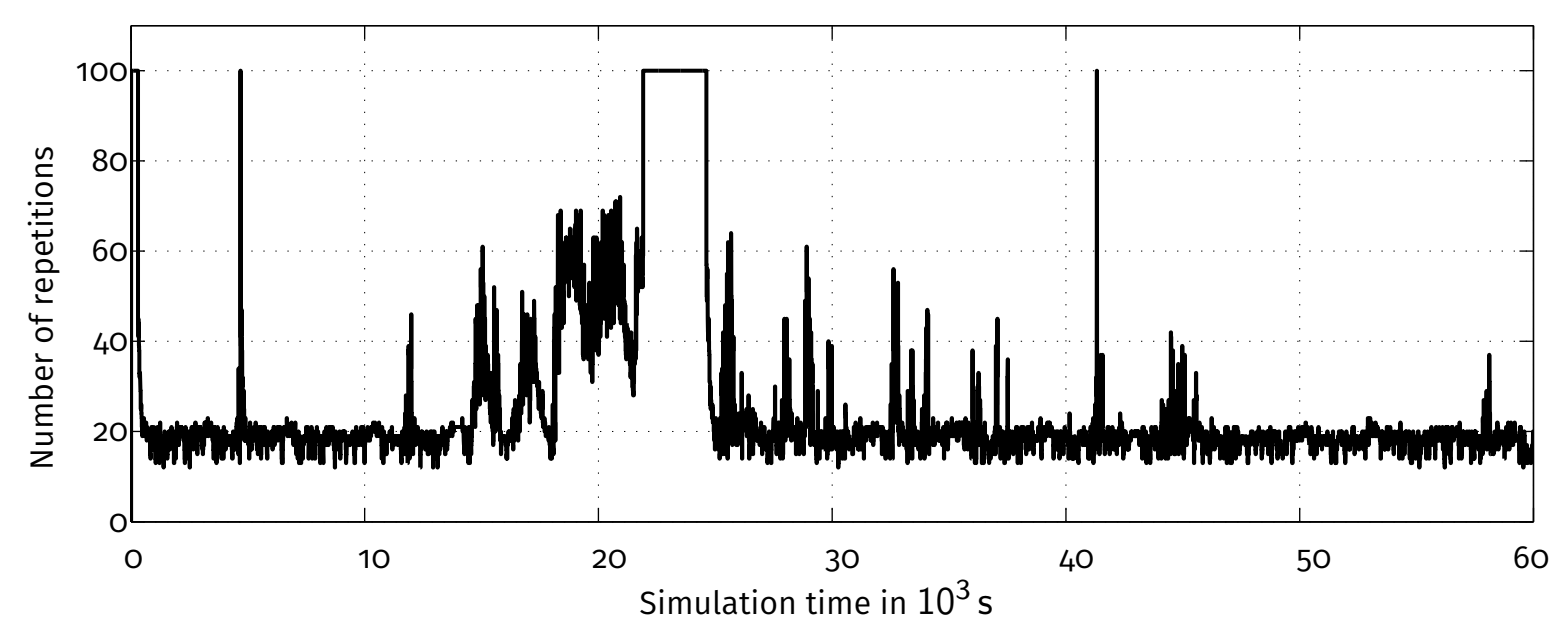

Figure 6.7. - Number of repetitions for the master controller (measurement file: 260820110447)

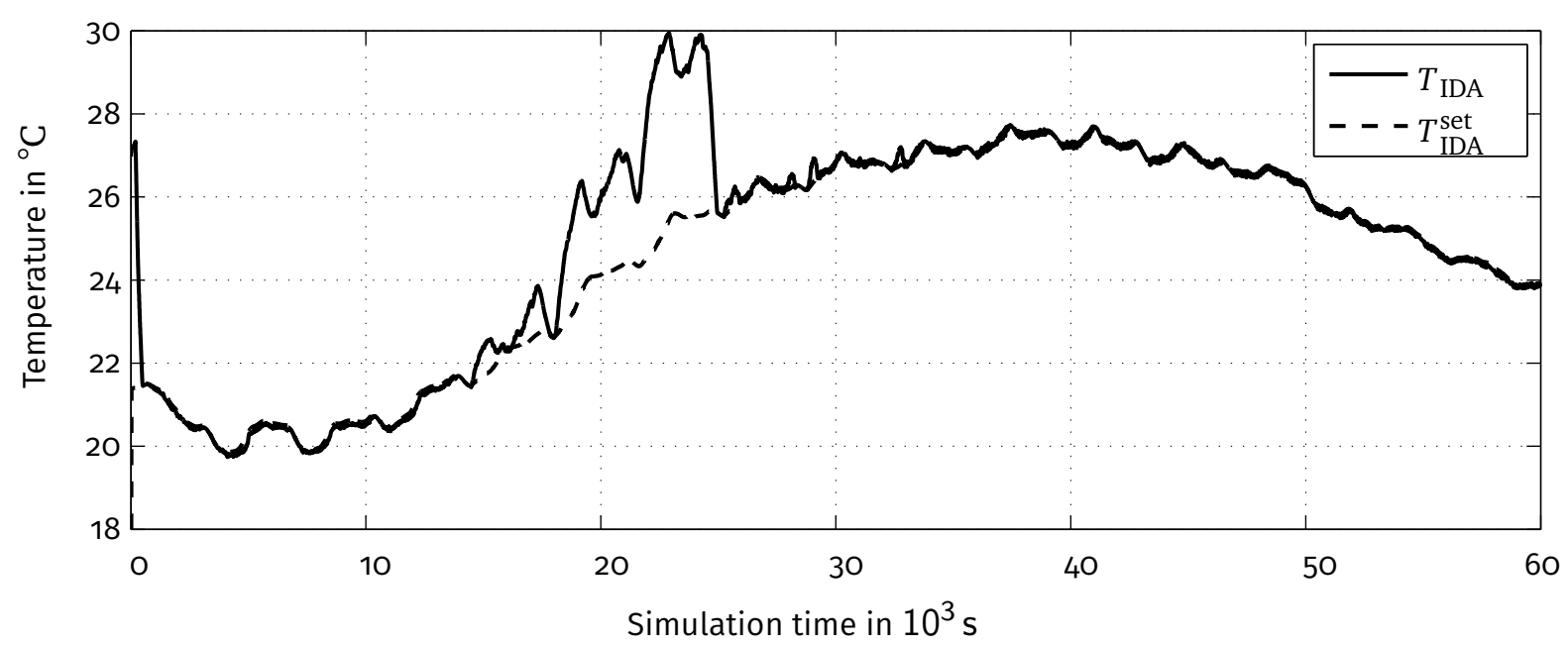

Figure 6.8. - Indoor air temperature of the simulated summer day (measurement file: 260820110447)

\subsubsection{Master controller}

For the MPC the weighting matrices $\mathbf{Q}$ and $\mathbf{R}$ need to be chosen adequately. In an online MPC exists another variable, i.e. the allowed calculation time. Normally, the allowed calculation time results in the number of allowed iterations or repetitions. These two values express the same, the allowed calculation time is easier to compare between different algorithms and the number of repetitions is easier to measure. So this section will use the number of repetitions.

The set up MPC has yet another parameter $\kappa$ which weights violations of the constraints. The solution approach deliberately accepts solutions that do not satisfy the constraints. Usually, these solutions are only intermediate results during a repetition. A combination of unfavourable circumstances (inadequate control variable and reaching the maximum number of repetitions) can lead to these intermediate results becoming the final result. This circumstance is shown on the basis of a hot summer day. The parameters of the HVAC are constant. 


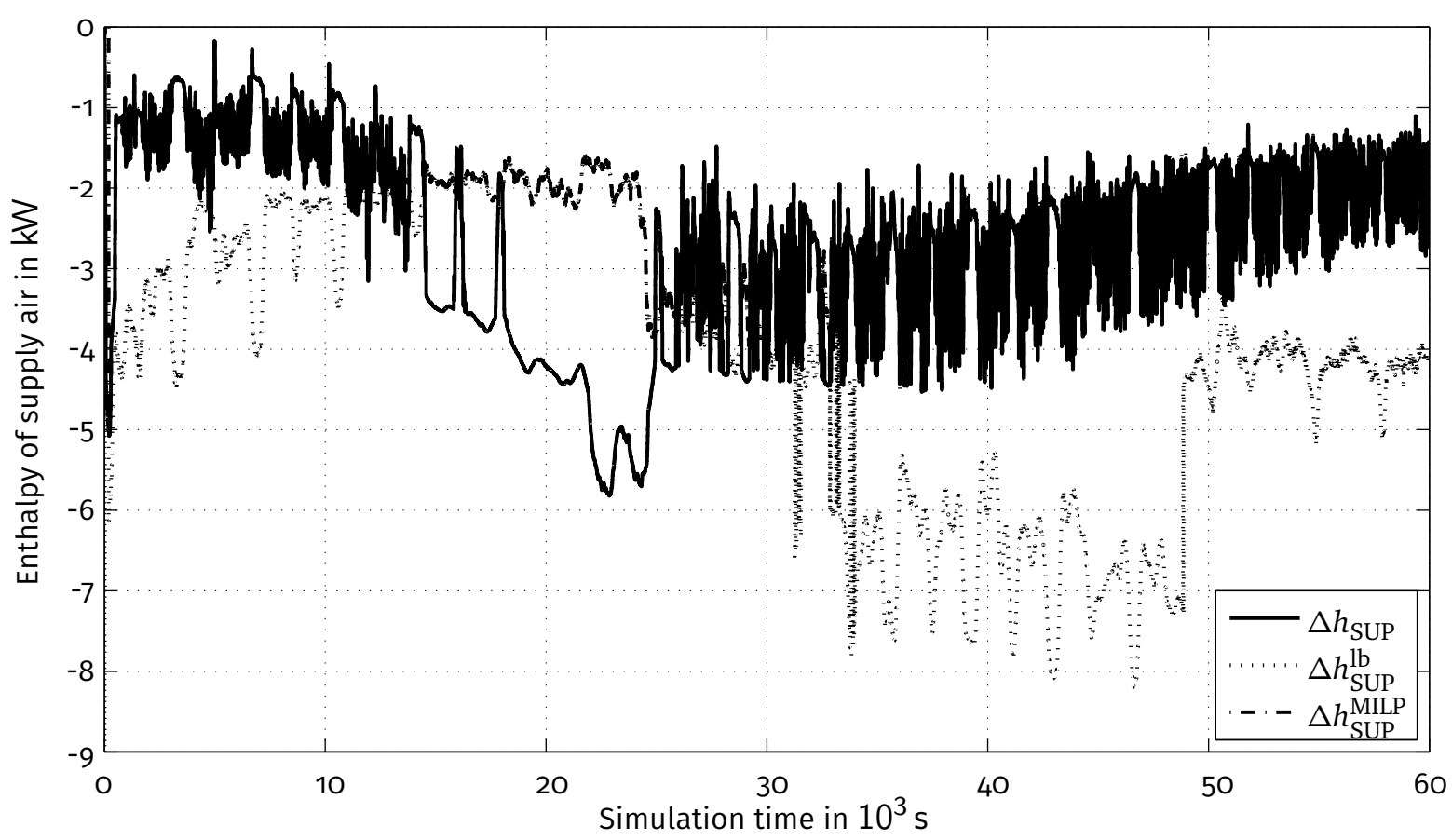

Figure 6.9. - Comparison for enthalpies of supply air for the master $\left(\Delta h_{\mathrm{SUP}}\right)$ and slave controller $\left(\triangle h_{S U P}^{M I L P}\right)$

Figure 6.7 shows the number of repetitions. Figure 6.8 shows the current value and the set-point of the IDA of the vehicle and in Figure 6.9 one recognises one of the control variables. The MPC sets $\Delta h_{\text {SUP }}, \Delta h_{\text {SUP }}^{\mathrm{lb}}$ is the lower bound and the slave controller converts it into the control variable $\Delta h_{\mathrm{SUP}}^{\mathrm{MILP}} . \Delta h_{\mathrm{SUP}}^{\mathrm{ub}}$ does not matter in this example. The controller achieves an excellent result during the first $10000 \mathrm{~s}$. Figure 6.8 shows no deviations between the set point and the current value. The number of repetitions is low. $\Delta h_{\text {SUP }}^{\mathrm{lb}}$ increases as well because of the increasing outdoor air temperature. For the first period, the controller satisfies the constraints, a control error is not (yet) visible. From $15000 \mathrm{~s}$ on, the MPC violates the constraints considerably (Figure 6.9), the number of repetitions increases and a control error arises which is still small. From $21000 \mathrm{~s}$ on, the control error violates the constraint on the indoor air temperature of $2{ }^{\circ} \mathrm{C}$. The number of repetitions reaches its maximum number. The solution quality is inadequate if the controller reaches the maximum number of repetitions during a longer time period. However, the controller returns to proper operation after this period, which is of high importance. As shown in Figure 6.9, the slave controller always satisfies the constraints on the control variable.

\subsubsection{Slave controller}

Results for the solution approach of full enumeration and heuristic are shown for the slave controller. Section 5.4.5 describes the used method. 


\section{Full enumeration}

Figure 6.10 shows the full enumeration of the MILP with constant COP. The enthalpy of the supply air $\Delta h_{\text {SUP }}$ in $\mathrm{kW}$ is plotted against the $\mathrm{x}$-axis and the energy consumption against the y-axis, also in $\mathrm{kW}$. To recognise the individual solutions, the number of drawn solution candidates $\mathscr{U}$ was reduced, which it eases to distinct the modes of operation. So one recognises from left to right:

Cooling - C

$\times$ Dehumidification - D

- Ventilation - V

* Heating (el) - E

+ Heating $(C R M)-\mathrm{H}$

- Heating (el+CRM) - B

Figure 6.11 shows that individual modes are not strictly separated any more because of the use of the $Q_{\mathrm{ac}} / Q_{\mathrm{hp}}$ performance map.

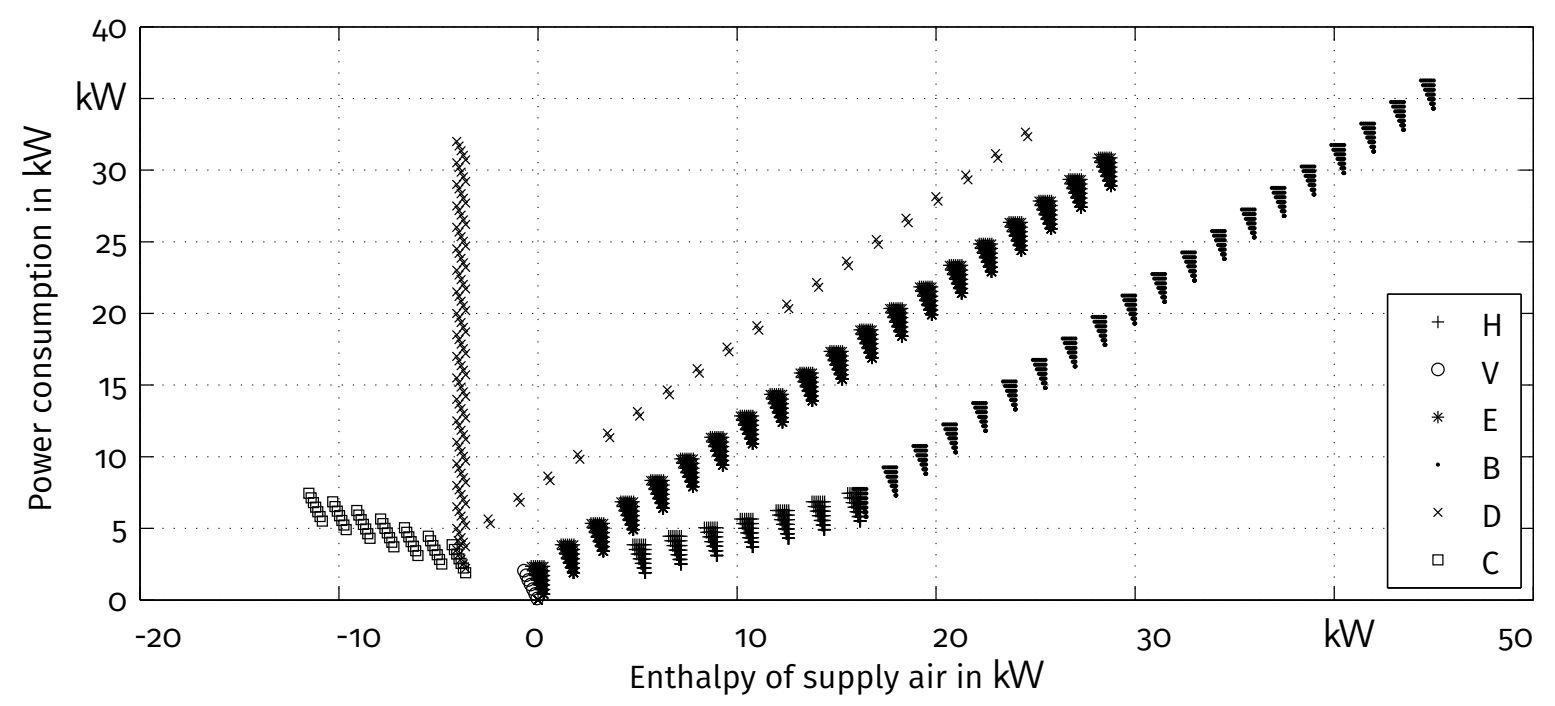

Figure 6.10. - Full enumeration of the MILP with constant COP

Heuristic

Due to the use of the heuristic, one only needs to search a small part of the solution set. Two examples (one summer and one winter day) show how the heuristic works. Figure 6.12 (the upper figure) depicts the boundaries of the modes, as seen in Figure 6.10. The figure does not show the modes Cooling and Dehumidification because they are not possible on the shown winter day. The figure below of Figure 6.12 magnifies the relevant P-h range to realise the individual solutions. Only a few solutions need to be calculated.

One recognises the same behaviour for a summer day in Figure 6.13. The Dehumidification mode supplies the needed enthalpy because compensating by electric heater is more efficient than delivering a higher mass flow of (warm) outdoor air. In general, experts consider using the dehumidification mode as a mistake by the controller. For the given conditions, however, it is the 


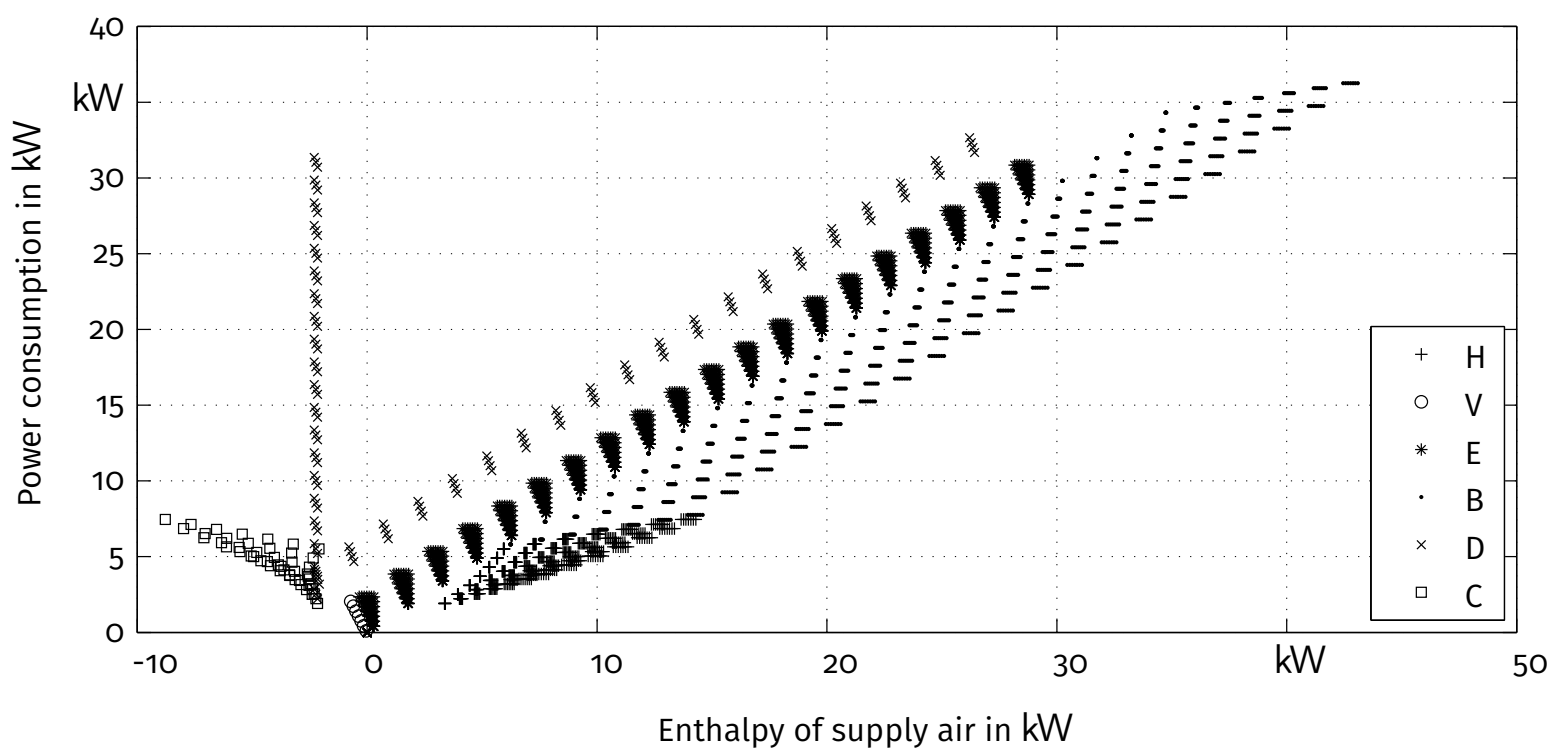

Figure 6.11. - Full enumeration of the MILP with variable COP

most efficient way. 

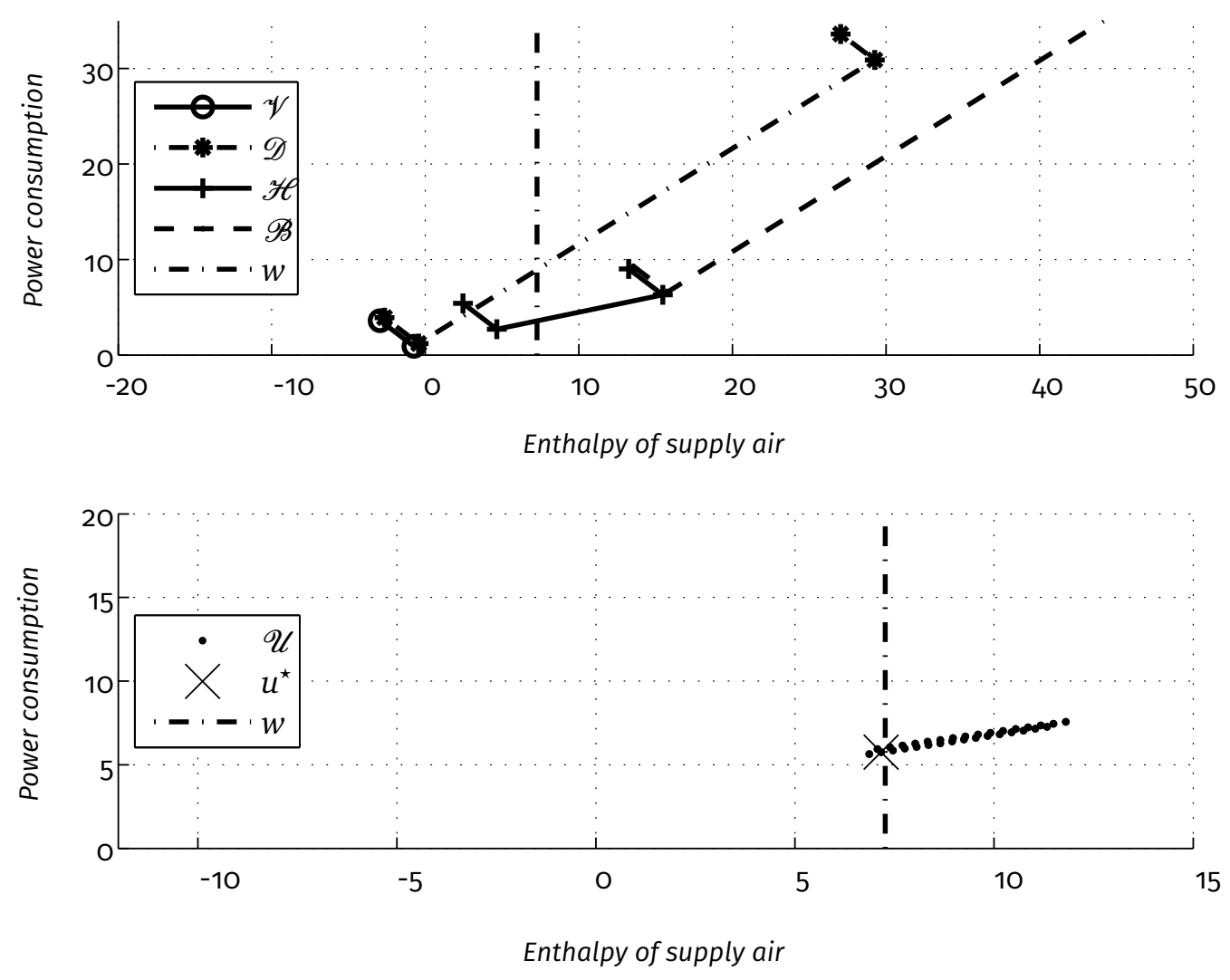

Figure 6.12. - 2D MILP of a winter day 

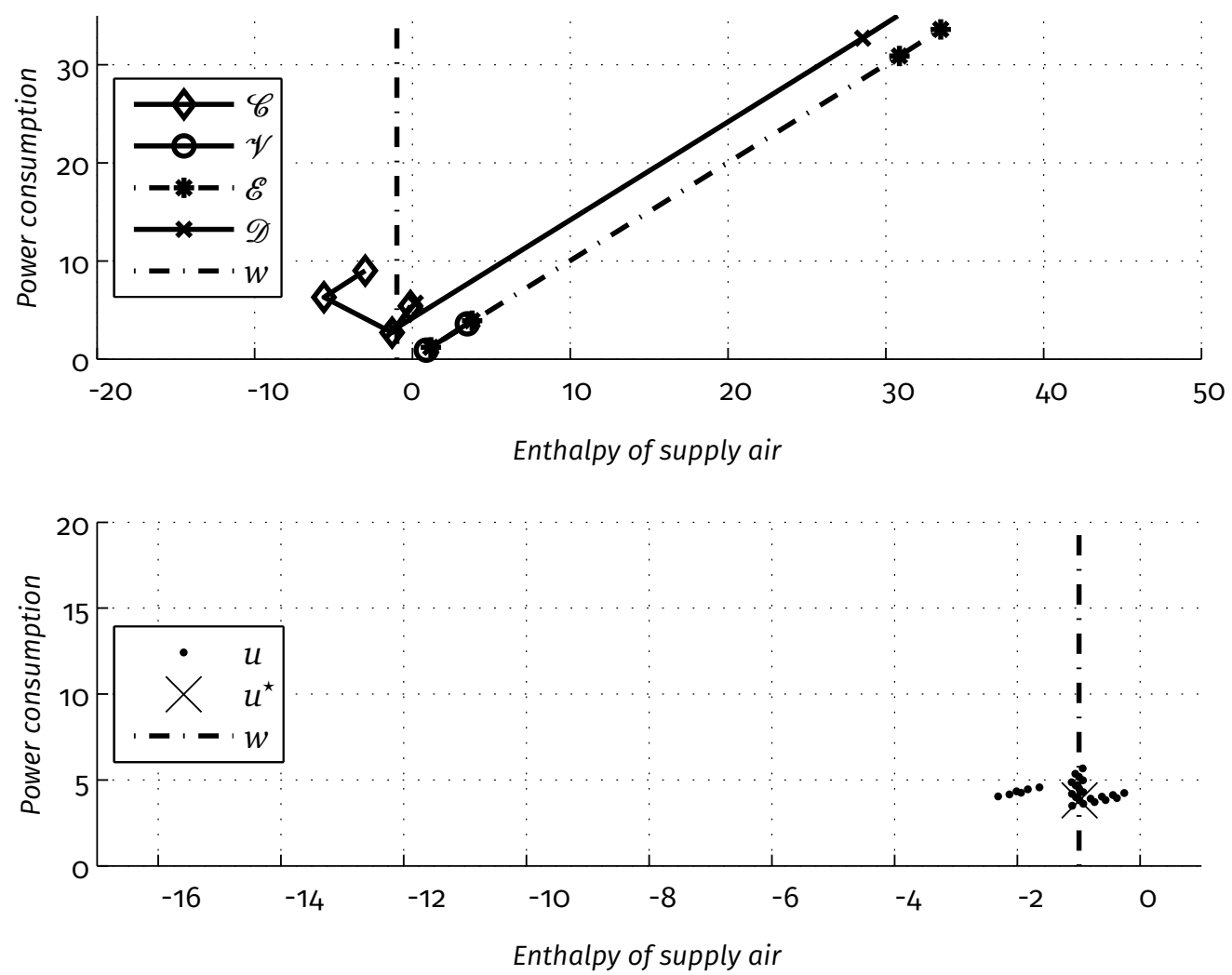

Figure 6.13. -2D MILP of a summer day 


\subsection{Controller Validation}

Hofstadter et al. [57] already presented the results shown in this section.

Because of the extent of the determination and the necessary description of the achieved results on the one hand and the low relevance to the controller validation on the other hand, the determination of the performance map is described in Appendix A.3.

The tram was transported to the CWT in Vienna. The testing institute contains two climatic wind tunnels, in which the larger one has a test section of $100 \mathrm{~m}$ and the smaller one a test section of about $34 \mathrm{~m}$ in length. The larger one allows wind speeds of up to $300 \mathrm{~km} / \mathrm{h}$, and the maximum wind speed of the smaller wind tunnel is $120 \mathrm{~km} / \mathrm{h}$, more than enough for tram tests. Both tunnels allow solar simulation from $250 \mathrm{~W} / \mathrm{m}^{2}$ to $1000 \mathrm{~W} / \mathrm{m}^{2}$, a temperature range of $-45^{\circ} \mathrm{C}$ to $60^{\circ} \mathrm{C}$ and a humidity range of $10 \%$ to $98 \%$. [44]

The CWT's team equipped the tram with several temperature, humidity, and energy sensors as well as load simulators during preparation. The tram underwent many experiments confirming the overall system (tram, HVAC, and controller) during a twelve-day measurement campaign. More than 100 experiments were conducted in the climatic wind tunnel, for measuring the performance map, for validating the HVAC and the controller. [96] provides a more detailed description. This subsection discusses the relevant measurements for the controller.

\subsubsection{Individual tests}

This section exemplifies six individual tests, four stationary tests and two dynamic tests. Usually, much effort is put into testing the HVAC's limits that are agreed by contract. However, these limits hardly occur in daily operation. The presented energy-saving HVAC needs a different approach.

It is known from pretests that the HVAC works between its limits $\left(-20^{\circ} \mathrm{C}\right.$ to $\left.35^{\circ} \mathrm{C}\right)$. Testing focuses on the most frequent operating conditions. These conditions depend on the city, the train and on how it is operated. They cannot be generalized and must be specifically determined for other use cases.

The test schedule contains the following temperatures:

a) $-10^{\circ} \mathrm{C}$

A winter day in Vienna can be as cold as $-10^{\circ} \mathrm{C}$. The service time of the tram starts at 4:30 and ends at 0:30. The tram is hardly occupied at these times and due to building's shade there is almost no irradiation. The HVAC should use Heating (el) to temper the tram.

b) $5{ }^{\circ} \mathrm{C}$

This test shows the off-peak hours of a spring or autumn day. $5^{\circ} \mathrm{C}$ is also the lowest temperature at which the heat pump can be used reasonably. The HVAC should use Heating (CRM) to temper the tram.

c) $20^{\circ} \mathrm{C}$

Conditions of a spring or autumn day are tested here. $20^{\circ} \mathrm{C}$ is the most common outdoor air temperature. The average number of passengers is 42. (All seats are taken). The HVAC should just use Ventilation or Cooling. Dehumidification should be avoided. 
Table 6.2. - Listing of current and new experiments for the calculation of the energy consumption. The mean value of the $0^{\circ} \mathrm{C}$ und $10^{\circ} \mathrm{C}$ experiments is the reference for $5^{\circ} \mathrm{C}$.

\begin{tabular}{cccccc}
\hline No. & $T_{\text {ODA }}$ & Load simulation & Sun simulation & actual & new \\
\hline a & $-10^{\circ} \mathrm{C}$ & o Persons & $0 \mathrm{~W} / \mathrm{m}^{2}$ & v13 & v68 \\
b & $5^{\circ} \mathrm{C}$ & o Persons & $0 \mathrm{~W} / \mathrm{m}^{2}$ & mean(v2,v78) & v71 \\
c & $20^{\circ} \mathrm{C}$ & 42 Persons & $0 \mathrm{~W} / \mathrm{m}^{2}$ & v24 & v101 \\
d & $28^{\circ} \mathrm{C}$ & 42 Persons & $0 \mathrm{~W} / \mathrm{m}^{2}$ & v28 & v82 \\
e & $0{ }^{\circ} \mathrm{C}-28^{\circ} \mathrm{C}$ & O Persons & $0 \mathrm{~W} / \mathrm{m}^{2}$ & v44 & v91 \\
f & $28^{\circ} \mathrm{C}-0^{\circ} \mathrm{C}$ & o Persons & $700 \mathrm{~W} / \mathrm{m}^{2}$ & v42 & v89 \\
\hline
\end{tabular}

d) $28^{\circ} \mathrm{C}$

These are the conditions of a summer day with the average number of passengers in the tram.

e, f) $0^{\circ} \mathrm{C}$ to $28^{\circ} \mathrm{C}$ respectively $28^{\circ} \mathrm{C}$ to $0^{\circ} \mathrm{C}$

Show (almost) the complete operating range of the HVAC in a short testing time. The HVAC should choose the correct HVAC mode over the entire range.

Table 6.2 shows environmental conditions of the conducted tests.

Table 6.3. - Resulting energy consumption of conducted tests.

\begin{tabular}{|c|c|c|c|c|c|c|c|c|c|c|c|}
\hline \multirow{2}{*}{\multicolumn{2}{|c|}{ No. }} & \multirow{2}{*}{$\begin{array}{r}P_{\text {fan }} \\
\mathrm{kW}^{1}\end{array}$} & \multirow{2}{*}{$\begin{array}{l}P_{\text {eh }} \\
\mathrm{kW}^{1}\end{array}$} & \multirow{2}{*}{$\begin{array}{l}P_{\text {comp }} \\
\mathrm{kW}^{1}\end{array}$} & \multirow{2}{*}{$\begin{array}{l}P_{\text {tot }} \\
\mathrm{kW}^{1}\end{array}$} & \multirow{2}{*}{$\begin{array}{l}\text { Saving } \\
\%\end{array}$} & \multirow{2}{*}{\multicolumn{5}{|c|}{\begin{tabular}{ccccccccc} 
& \multicolumn{3}{c}{$P_{\mathrm{fan}}$} & \multicolumn{3}{c}{$P_{\mathrm{HC}}$} & \multicolumn{3}{c}{$P_{\text {comp }}$} \\
0 & 1 & 2 & 3 & 4 & 5 & 6 & $\mathrm{~kW}^{1}$ & 8
\end{tabular}}} \\
\hline & & & & & & & & & & & \\
\hline \multirow[b]{2}{*}{$a$} & current & 0.84 & 6.89 & 0 & 7.79 & - & \multirow{2}{*}{\multicolumn{5}{|c|}{ 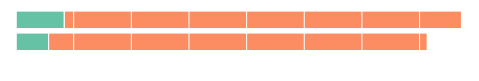 }} \\
\hline & new & 0.57 & 6.57 & 0 & 7.14 & 9 & & & & & \\
\hline \multirow{2}{*}{ b } & current & 0.90 & 2.98 & 0 & 3.88 & - & \multirow{2}{*}{\multicolumn{3}{|c|}{ || }} & & \\
\hline & new & 1.07 & 0 & 1.57 & 2.64 & 32 & & & & & \\
\hline \multirow{2}{*}{$C$} & current & 1.13 & 0.03 & 1.70 & 2.86 & - & \multirow{2}{*}{\multicolumn{3}{|c|}{ 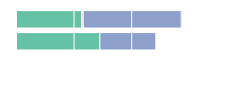 }} & & \\
\hline & new & 1.45 & 0 & 0.97 & 2.42 & 15 & & & & & \\
\hline \multirow{2}{*}{$d$} & current & 1.21 & 0.01 & 2.81 & 4.03 & - & \multirow{2}{*}{\multicolumn{3}{|c|}{ |l||l|| }} & & \\
\hline & new & 1.12 & 0 & 2.62 & 3.82 & 5 & & & & & \\
\hline \multirow{2}{*}{ e } & current & 0.90 & 1.27 & 0.26 & 2.43 & - & \multirow{2}{*}{ || } & & & & \\
\hline & new & 0.85 & 0.43 & 0.81 & 2.09 & 14 & & & & & \\
\hline \multirow{2}{*}{ f } & current & 0.94 & 1.05 & 0.88 & 2.87 & - & \multirow{2}{*}{ 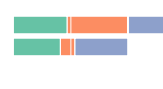 } & & & & \\
\hline & new & 0.82 & 0.26 & 0.92 & 1.99 & 31 & & & & & \\
\hline
\end{tabular}

${ }^{1}$ Energy consumption of the component as mean value per hour in $\mathrm{kW} \mathrm{h/h}$

Figures for each test show the IDA temperature time series against the test time for each test. Besides the mean temperature $T_{\text {IDA }}$, hey also show the minimum and the maximum temperatures $\left(T_{\mathrm{IDA}}^{\mathrm{lb}}\right.$ respectively $T_{\mathrm{IDA}}^{\mathrm{ub}}$ ) at different measuring positions of the tram. The temperature range that satisfies the thermal comfort has a white background, everything else has a grey background. The 
shown signals are temperature measurements of the CWT. Measurements from the HVAC used for control are not available. This phenomenon shows the problem of inhomogeneous temperature distribution in the passenger compartment.

In addition, the power consumption of the individual parts (fan, compressor, and electric heater) as well as the overall power consumption are listed as hourly mean energy consumption in $\mathrm{kWh} / \mathrm{h}$ for every test. Reference values are taken from measurements conducted on the same vehicle without modifications two years earlier. Results are presented graphically and tabularly (Table 6.3).

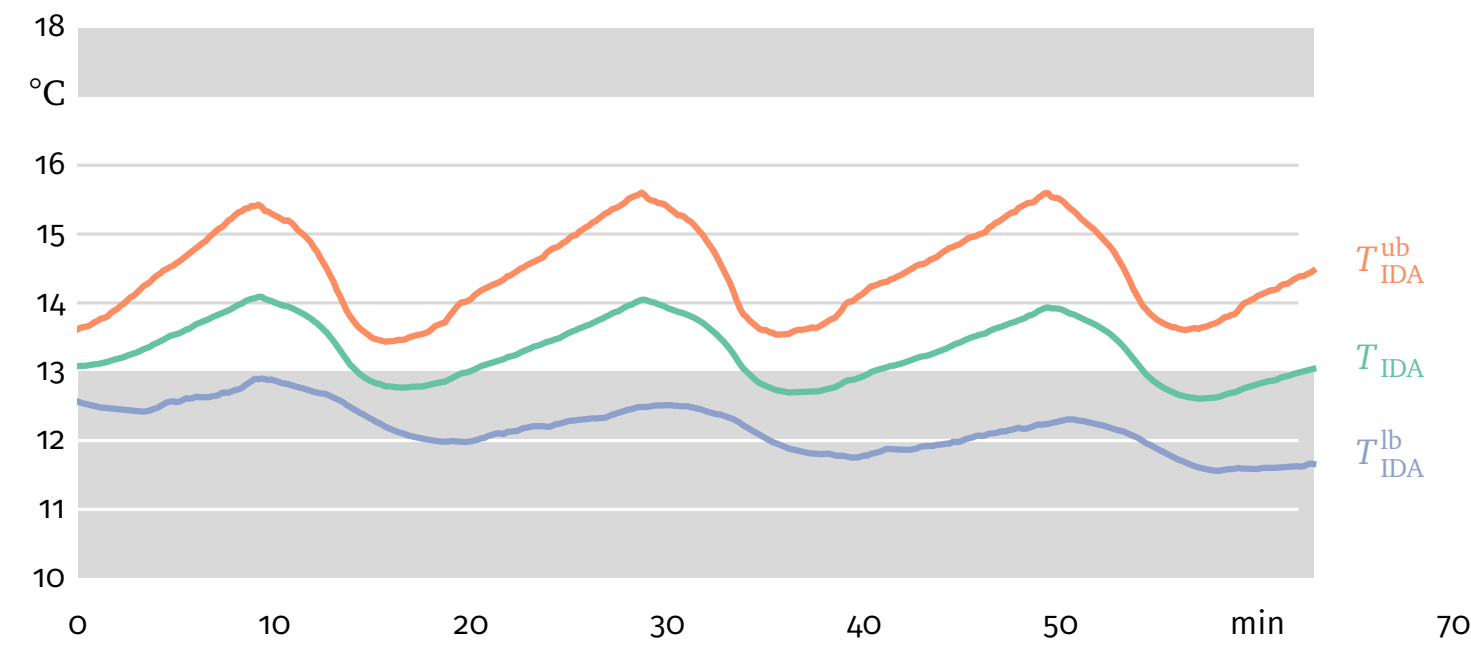

Figure 6.14. - Test conditions: $T_{\mathrm{ODA}}=-10^{\circ} \mathrm{C}$, Load simulation: o persons, sun simulation: $0 \mathrm{~W} / \mathrm{m}^{2}$

Figure 6.14 shows the mean IDA temperature varying by $1.5^{\circ} \mathrm{C}$ just above the limit. Only for short periods, the mean temperature is below the limit. The IDA range is moderate with $2{ }^{\circ} \mathrm{C}$ to $3{ }^{\circ} \mathrm{C}$. The pre-emptive controller can take advantage of the fine-grained control possibilities, which allows the supply air fan to reduce the mass flow. The total energy consumption is reduced by $9 \%$ or $0.65 \mathrm{kWh} / \mathrm{h}$.

b

The used Archimedean controller successfully suppresses Heating (el) by switching between Heating (CRM) and Ventilation modes. It achieves an acceptable mean temperature and an energy saving of $32 \%$ or $1.24 \mathrm{kWh} / \mathrm{h}$. However, the temperature varies by $4{ }^{\circ} \mathrm{C}$ in $20 \mathrm{~min}$, which might be unpleasant for the passengers (see Figure 6.15). More over, the IDA temperature range increases rapidly from (almost) zero to $3^{\circ} \mathrm{C}$ in $20 \mathrm{~min}$.

C

The Archimedean controller successfully suppresses Dehumidification by switching between Cooling and Ventilation. The achieved mean temperature is acceptable and during alternating the 

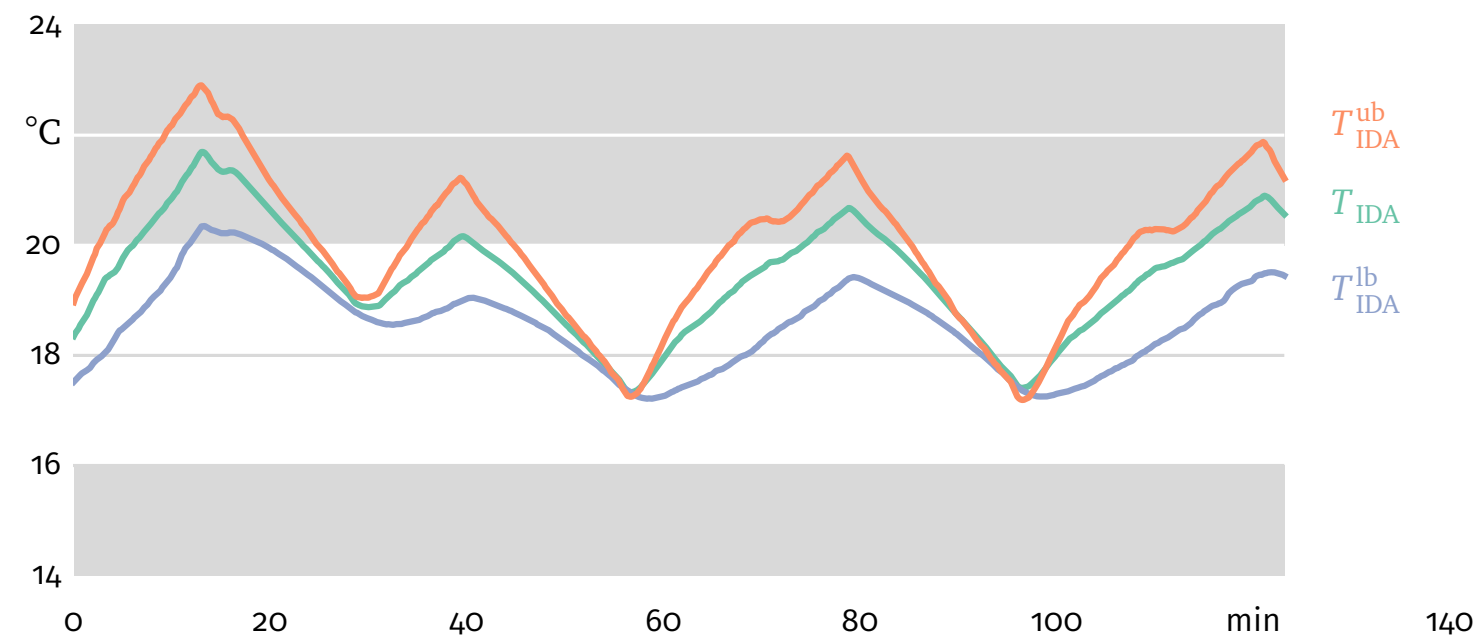

Figure 6.15. - Test conditions: $T_{\mathrm{ODA}}=5^{\circ} \mathrm{C}$, Load simulation: o persons, sun simulation: $0 \mathrm{~W} / \mathrm{m}^{2}$

temperature varies only by $0.7^{\circ} \mathrm{C}$ (see Figure 6.16). The mean IDA temperature is stable. $T_{\text {IDA }}^{\mathrm{ub}}$ slowly increases and the difference to the mean IDA also increases. This occurs because the tail section does not have its own HVAC but is supplied by the third HVAC. An energy saving of $15 \%$ or $0.44 \mathrm{kWh} / \mathrm{h}$ is achieved.

d

The energy saving is low with $5 \%$ or $0.206 \mathrm{kWh} / \mathrm{h}$, but the HVAC works near its design point during testing. So, no improvement was expected. Figure 6.17 shows that the temperature varies by $1.5^{\circ} \mathrm{C}$. The temperature constraints are violated several times, the violation is quite small $\left(0.7^{\circ} \mathrm{C}\right)$. The tail effect described in c) is present again.

e

The proper controller is always used and switching between both controllers works smoothly. Thermal comfort constraints are satisfied, except during switching between Heating (CRM) and Ventilation (as seen in No. b). Figure 6.18 shows Heating (el) during the first hour. The HVAC alternates between Ventilation and Heating (CRM) for the next four hours. Then the HVAC ventilates for about an hour until it alternates between Ventilation and Cooling. The achieved energy saving is $14 \%$ or $0.332 \mathrm{kWh} / \mathrm{h}$.

Switching between new controllers works smoothly again. The thermal comfort is near the upper end of the feasible range, but is acceptable. Figure 6.19 shows alternating between Cooling and Ventilation, then a distinctive Ventilation phase and again alternating between Heating (CRM) and Ventilation at the end. There is only a short Heating (el) phase in the end. Energy saving is as high as $31 \%$ or $0.874 \mathrm{kWh} / \mathrm{h}$. 

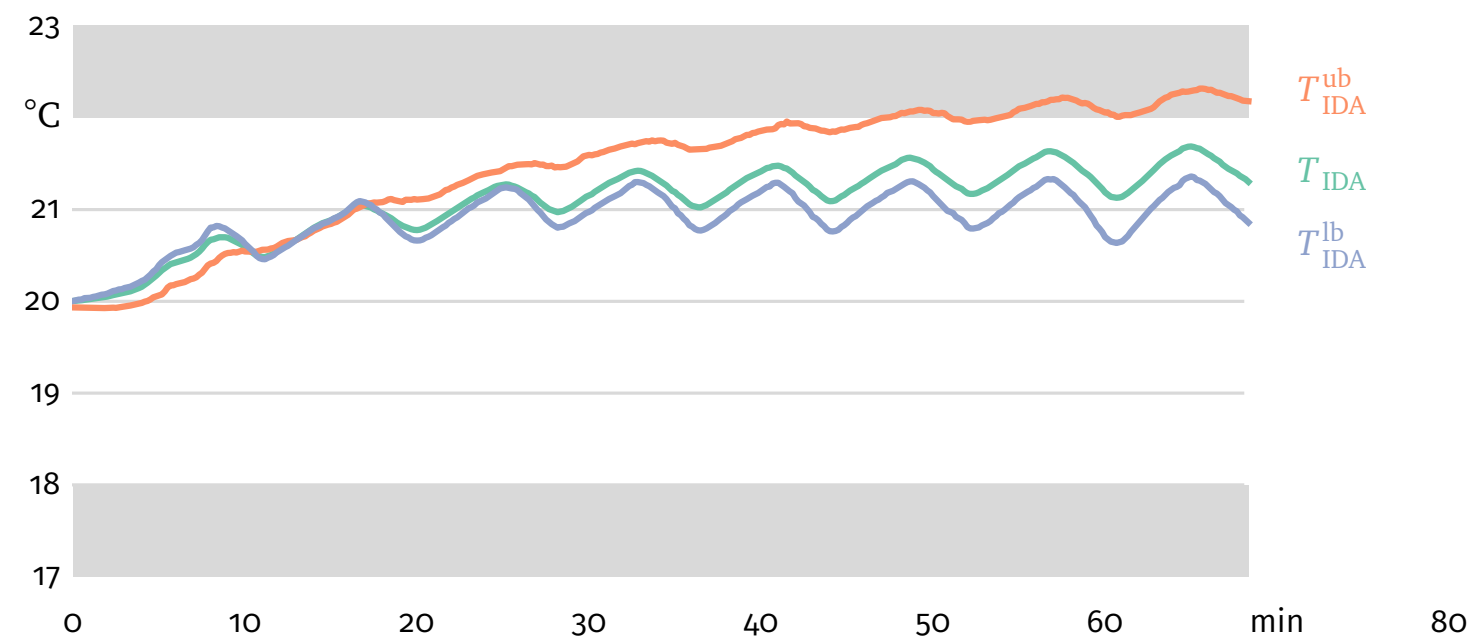

Figure 6.16. - Test conditions of v101 $T_{\text {ODA }}=20^{\circ} \mathrm{C}$, Load simulation 42 persons, sun simulation $0 \mathrm{~W} / \mathrm{m}^{2}$

\subsubsection{Annual energy consumption}

The calculation of the annual energy consumption for the actual [95] and the new vehicle [96] was done in both cases according to a method by proposed by Richter [97]. In doing so, the proposed method of [97] was used to calculate the annual energy consumption from individual measurements.

To apply this method, it is necessary to measure several temperature ramps $\left(0^{\circ} \mathrm{C}\right.$ to $28^{\circ} \mathrm{C}$ at different solar radiation intensities $\left(0 \mathrm{~W} / \mathrm{m}^{2}\right.$ and $\left.700 \mathrm{~W} / \mathrm{m}^{2}\right)$ and different load levels $(0$ persons and 42 persons). From individual experiments a weighted mean value is calculated. One obtains the power consumption of the vehicle versus the ODA temperature. Figure 6.20 shows the power consumption of the actual and the new system. The energy saving of the new system compared to the old one are plotted in Figure 6.21 versus the ODA temperature. The power consumption of the individual ODA temperatures is now weighted with the absolute frequency of the temperatures in one year, to calculated the annual energy consumption. Taking into account the temperature distribution of the years 2010/2011 an annual savings of $26 \%$ follows, this correspond to about $8500 \mathrm{~kW} \mathrm{~h}$ per train [96]. [96] writes, that without consideration of the actual annual temperature distribution savings of $32 \%$ follow.

\subsubsection{Conclusions of the controller validation}

Transport accounts for much of the overall energy consumption, and the second largest energy consumer in rail vehicles is the HVAC. Energy saving HVACs (including demand-based ventilation, heat pumps and fine-grained control of their parts) need a proper control because of the increased difficulty of the control problem. This paper models for all aspects of the thermal comfort problem in trams (trams, HVAC and thermal comfort) and proposes a controller design of two different approaches. The first approach splits the control problem to facilitate calculation. An MPC controls the tram model and calculates an auxiliary control variable. Then a method based on integer linear 


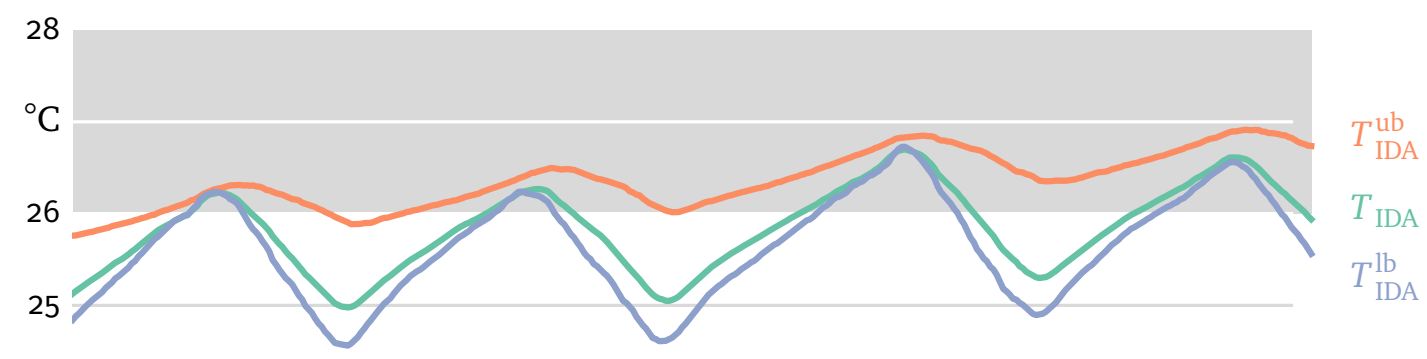

24

23

22

30

40

50

$\min$

70

Figure 6.17. - Test conditions: $T_{\mathrm{ODA}}=28^{\circ} \mathrm{C}$, Load simulation 42 persons, sun simulation $0 \mathrm{~W} / \mathrm{m}^{2}$

optimisation calculates switching commands for the HVAC. The second approach prevents only unwanted conditions using a model predictive control based method. All libraries and algorithms were set up on the dSpace Autobox and built into a tram. The Viennese climatic wind tunnel tested the train during a twelve-day measuring campaign. Individual tests showed savings from $9 \%$ to $32 \%$ and an estimated annual energy saving of $32 \%$. The research has proved that the proposed approach works well, although, it needs minor adjustments (satisfy constraints and prevent overshooting during the Archimedean approach) for broad application. 


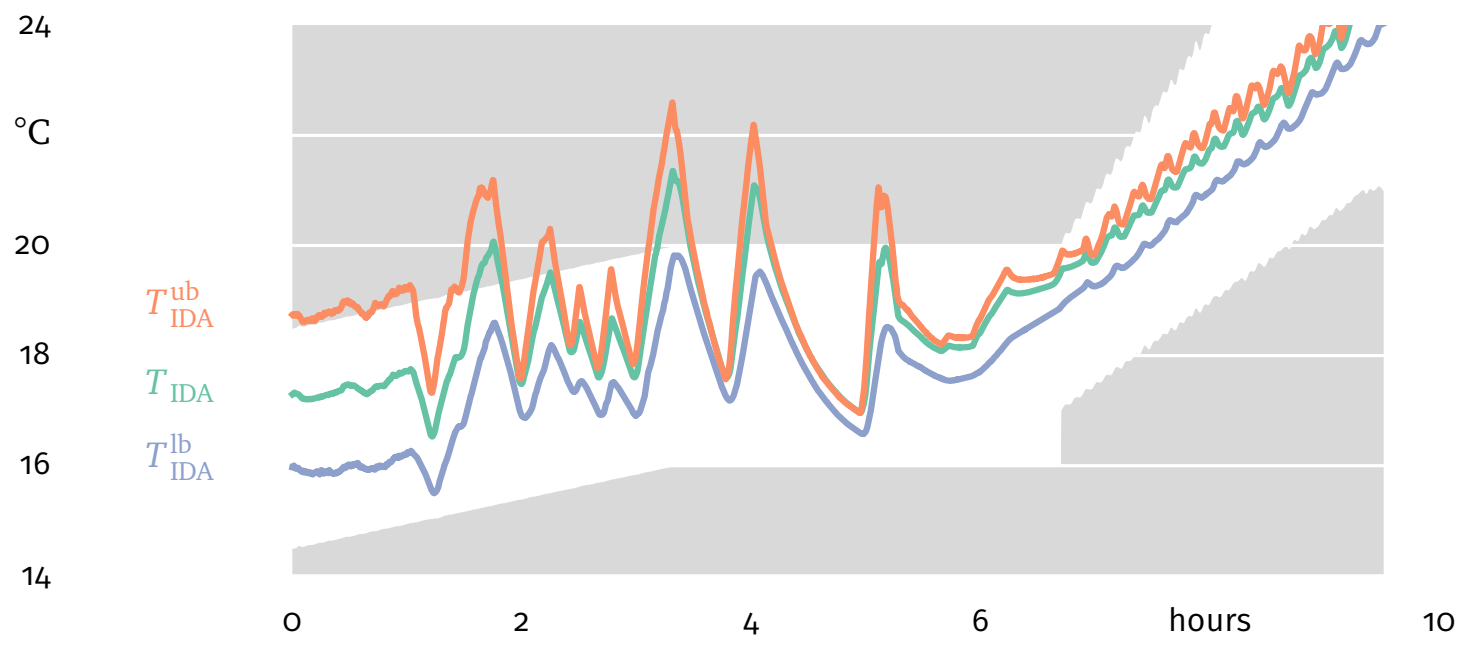

Figure 6.18. - Test conditions: $T_{\text {ODA }}=0{ }^{\circ} \mathrm{C}$ to $28^{\circ} \mathrm{C}$, Load simulation o persons, sun simulation $0 \mathrm{~W} / \mathrm{m}^{2}$

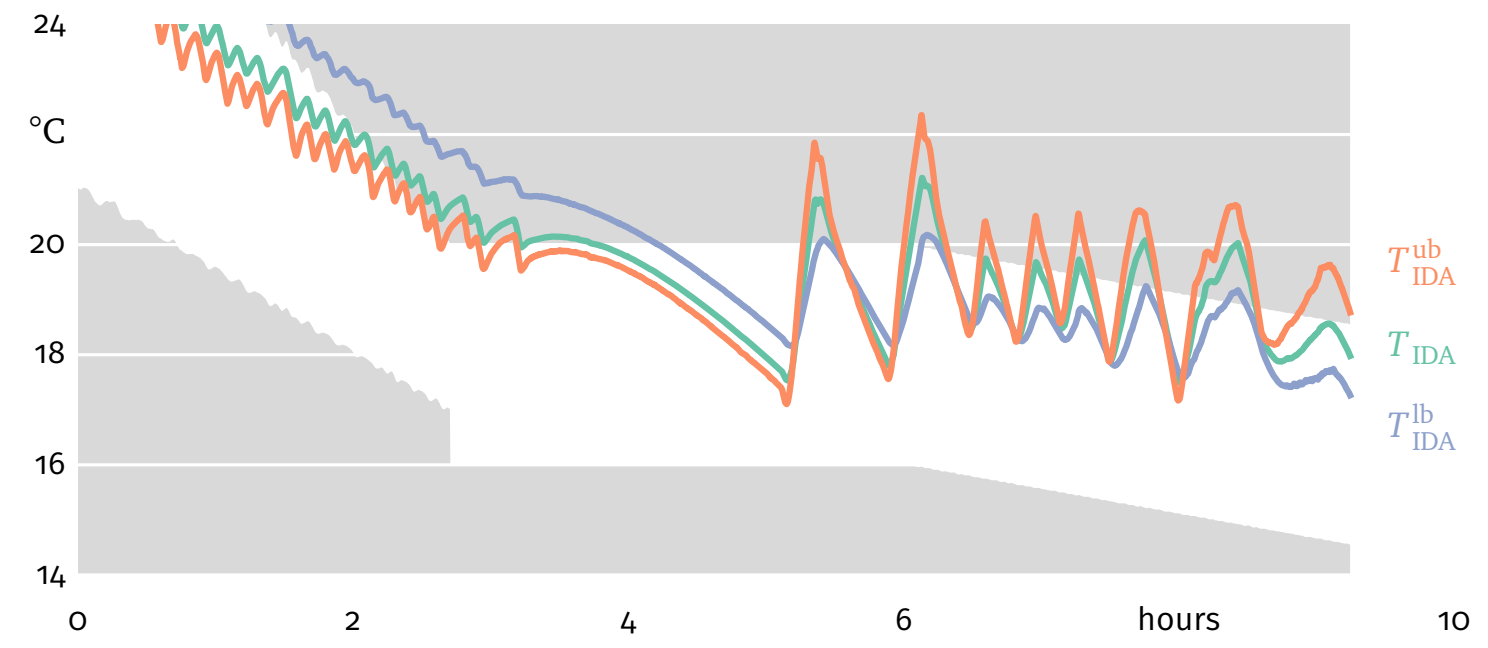

Figure 6.19. - Test conditions: $T_{\mathrm{ODA}}=0{ }^{\circ} \mathrm{C}$ to $28^{\circ} \mathrm{C}$, Load simulation o persons, sun simulation $700 \mathrm{~W} / \mathrm{m}^{2}$ 


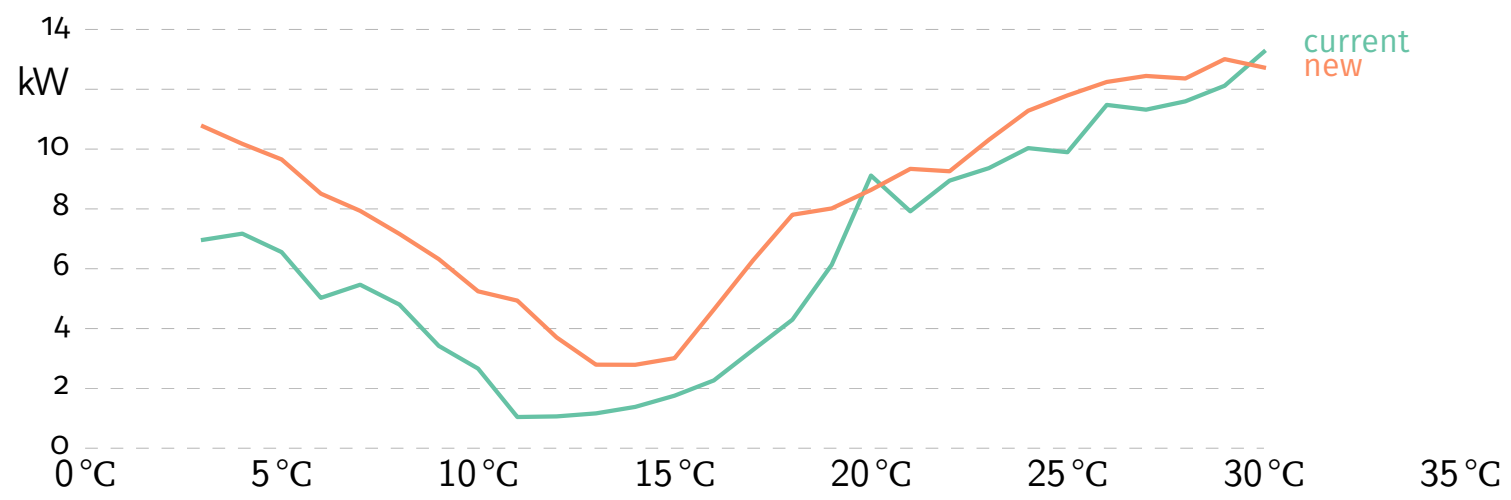

Figure 6.20. - Comparison of the representative power consumption of HVACs between the current and the new system plotted against the outdoor air temperature

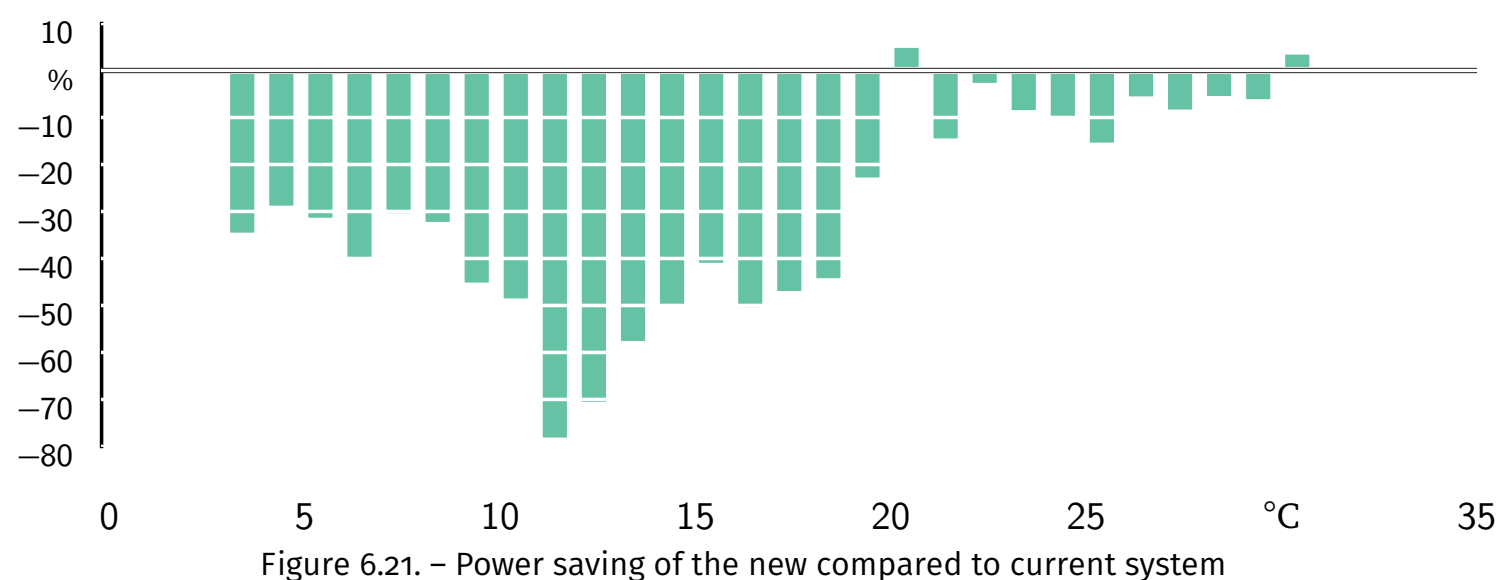

Figure 6.21. - Power saving of the new compared to current system 



\section{Outlook}

The discussed control concept has achieved improved solutions for the problem of thermal comfort. However, further questions have arisen or it became clear, that accompanying measures could even further improve the control result. These measures include:

Design $\neq$ maximum conditions Acceptance test check the maximum values of the permissible working conditions. Normally these maximum values do not agree with the usual working conditions. So, the resulting air conditioning works in the entire range but the performance for often occurring conditions is random.

Producers should design their air conditioning for often occurring conditions and not for rarely occurring maximum values. Indeed, the Producer must guarantee a save operation for the entire working range. But acceptance tests should also check often occurring conditions. This needs detailed knowledge of (future) environmental conditions because simple estimations do not suffice.

A dynamic air conditioning model A dynamic air conditioning model could further improve the control result. The current approach needs some assumptions. A dynamic model allows reducing these (conservative) assumptions.

Systematic function tests An air conditioning undergoes detailed and systematic functional checks and tests before the producer delivers it. This allows the producer to find and correct errors as early as possible. The controller should undergo the same functional tests. A hardware in the loop test stand can perform these checks.

Representative measurement of the indoor air temperature Measuring the representative indoor air temperature of the train by the air conditioning is difficult. Usually, a more or less constant error arises independence of different variables and climatic wind tunnel experiments allow to estimate them. Because of the higher complexity of the air conditioning this (constant) correction is not enough. A measurement of the indoor air temperature which better approaches the reference measurement is necessary.

Modelling of the air duct During designing the model the author assumed, that air conditions of the air entering the train, are identical to the air conditions of air leaving the air conditioning. So, this work neglects the air ducts. This leads to a systematic error for conventional air conditioning 
but it is roughly the same for all working modes. Because of the variable airflow of the new air conditioning, the delay time of air in the air ducts changes and for low airflows the described error increases. Air conditions with variable airflow must consider the air ducts.

Guaranteed minimum energy consumption The current control concept, consisting of a master and a slave controller, guarantees that air conditioning systems achieve the smallest possible energy consumption for the current moment. However, over the control horizon there might be a solution with a lower energy consumption and nevertheless satisfies the constraints. Figure 5.2 shows clearly, the solution with the lowest possible energy consumption is at the intersection of the Pareto front and the minimum permissible comfort. Thus, the only criterion for optimisation is minimising the energy consumption. Then, thermal comfort is an optimisation constraint. Although this theory led to critical discussions, it is the author's opinion that this theory allows for more energy savings. Equation (7.1) shows a sketch of the resulting formula. Currently, this problem is not solvable in 10 s with the available computational power of an air conditioning controller.

$$
\begin{aligned}
& \text { search for } x(i), u(i), u_{k}(i), x_{k}(i) \quad i=1 \ldots T \\
& \text { minimise } P_{e l}=\sum_{i=1}^{T} k_{k} \cdot x_{k}(i) \\
& \text { subject to thermal comfort } \\
& x(i)^{T} \mathbf{Q} x(i)+q x(i)-P M V^{l b} \leq 0 \\
& -x(i)^{T} \mathbf{Q} x(i)-q x(i)+P M V^{u b} \leq 0 \\
& \text { Vehicle model } \\
& x(i+1)=\mathbf{A} x(i)+\mathbf{B} u(i)+\mathbf{E} z(i)+v(i) \\
& M(i)= \begin{cases}V & u_{V}^{l b}(i) \leq u_{1}(i) \leq u_{V}^{u b}(i) \\
H & u_{H}^{l b}(i) \leq u_{1}(i) \leq u_{H}^{u b}(i) \\
C & u_{C}^{l b}(i) \leq u_{1}(i) \leq u_{C}^{u b}(i) \\
E & u_{E}^{l b}(i) \leq u_{1}(i) \leq u_{E}^{u b}(i) \\
B & u_{B}^{l b}(i) \leq u_{1}(i) \leq u_{B}^{u b}(i) \\
D & u_{D}^{l b}(i) \leq u_{1}(i) \leq u_{D}^{u b}(i)\end{cases} \\
& \text { control error } \\
& \left(u(i)-u_{k}(i)\right)^{2} \leq u_{e}^{u b} \\
& \text { HVAC } \\
& x_{k}(i)=g(M(i), x(i), u(i), z(i)) \\
& u_{k}(i)= \begin{cases}f_{V H E B}\left(x(i), x_{k}(i), z(i)\right) & V, H, E, B \\
f_{C D}\left(x_{k}(i), z(i)\right) & C, D\end{cases}
\end{aligned}
$$




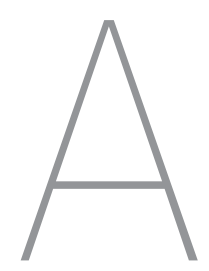

\section{Parameters}

This chapter lists more parameters for the used models and states the switching conditions of the FSM. Section 3.3 describes the HVAC and this section lists further parameters for the electric heater and the cooling cycle of the CRM. Assumptions made in Chapter 5 for the MILP are summarised in a table below.

\section{A.1. Finite state machine switching conditions}

This section describes the switching conditions of the FSM exhaustively. Section 3.4 explains the structure of the FSM. The following lists describes each switching conditions of the FSM

- Global FSM (Section 3.4.1),

- Electric heater (Section 3.4.2),

- Compressor (Section 3.4.3),

- Bypass valve (Section 3.4.4),

- Supply air fan (Section 3.4.5) and

- Cooling air fan (Section 3.4.6).

Table A.1. - Switching conditions of the global state machine

\begin{tabular}{cc}
\hline Description & Conditions \\
\hline B1, B4, B9 & status_switch is auto \\
B2, B6, B8 & status_switch is off \\
B3, B5, B7 & status_switch is preheat \\
B10 & $T_{O D A}>21$ and $\left(T_{O D A}>7\right.$ and $\left.T_{I D A}^{s e t}+2<T_{I D A}\right)$ \\
B11 & not B10 \\
\hline
\end{tabular}

Another variable $T_{S U P}^{\text {set }}$ need to be defined before the switching conditions of the electric heater FSM can be listed. It gives the set point temperature for the supply air and follows from

$$
T_{S U P}^{s e t}=\frac{1}{5}\left(T_{I D A}^{s e t}-T_{I D A}\right) T_{I D A}^{s e t}+\frac{5\left(T_{I D A}^{s e t}-T_{O D A}\right)}{T_{I D A}^{s e t}+20}+T_{I D A}^{s e t} .
$$


Table A.2. - Switching conditions of the electric heater state machine (SM)

\begin{tabular}{cl}
\hline Description & Conditions \\
\hline B1 & not $(\mathrm{B} 2$ or B4) \\
B2 & $T_{S U P}^{\text {set }}-T_{S U P}>4$ and $T_{I D A}<T_{I D A}^{\text {set }}$ and $\left(T_{O D A}<10\right.$ or $\left.T_{I D A}<10\right)$ \\
B4 & $T_{S U P}^{\text {set }}-T_{S U P}>3$ and $T_{I D A}<T_{I D A}^{\text {set }}$ \\
B5, B9 & $T_{S U P}^{\text {set }}-T_{S U P}>6$ and $T_{I D A}<T_{I D A}^{\text {set }}$ and $t_{i S} \geq 30 \mathrm{~s}$ \\
B7 & B2 and $t_{\text {iS }} \geq 30 \mathrm{~s}$ \\
B8, B14 & $\left(T_{S U P}^{\text {set }} \leq T_{S U P}\right.$ or $\left.T_{I D A} \geq T_{I D A}^{\text {set }}\right)$ and $t_{i S} \geq 30 \mathrm{~s}$ \\
B15 & $\left(T_{S U P}^{\text {set }}-T_{S U P}>6\right.$ and $T_{I D A}<T_{I D A}^{\text {set }}$ and $\left(T_{O D A} \geq 10\right.$ or $\left.\left.T_{I D A} \geq 10\right)\right)$ \\
B3, B6, B10, & and $t_{i S} \geq 30 \mathrm{~s}$ \\
B11, B12, B13, & False \\
B16 & \\
\hline
\end{tabular}

Table A.3. - Switching conditions of the compressor FSM

\begin{tabular}{cc}
\hline Description & Conditions \\
\hline B1 & $T_{I D A}>T_{I D A}^{\text {set }}+1$ and $T_{O D A}>10$ \\
B2 & $T_{I D A}<T_{I D A}^{\text {set }}-1$ \\
B3 & $T_{I D A}>T_{I D A}^{\text {set }}+1$ and $T_{O D A}>10$ and $t_{i S} \geq 300 \mathrm{~s}$ \\
B4 & $T_{I D A}<T_{I D A}^{\text {set }}-1$ and $t_{i S} \geq 60 \mathrm{~s}$ \\
\hline
\end{tabular}

Table A.4. - Switching conditions of the bypass valve FSM

\begin{tabular}{cc}
\hline Description & Conditions \\
\hline B1 & $\left(T_{I D A}<T_{I D A}^{\text {set }}\right.$ or $\left.T_{\text {SUP }} \leq 3\right)$ and $s_{\text {comp }}$ is 2 \\
B2 & not $\left(\left(T_{I D A}<T_{I D A}^{\text {set }}\right.\right.$ or $\left.T_{S U P} \leq 3\right)$ and $s_{\text {comp }}$ is 2$)$ \\
B3 & $\left(T_{I D A}<T_{I D A}^{\text {set }}\right.$ or $\left.T_{S U P} \leq 3\right)$ and $s_{\text {comp }}$ is 2 and $t_{i S} \geq 30 \mathrm{~s}$ \\
B4 & $\left(\left(T_{I D A}>T_{I D A}^{\text {set }}+1\right.\right.$ or $\left.T_{S U P} \geq 8\right)$ or $s_{\text {comp }}$ is 1$)$ and $t_{i S} \geq 60 \mathrm{~s}$ \\
\hline
\end{tabular}


Table A.5. - Switching conditions of the supply air fan FSM

\begin{tabular}{ccc}
\hline & \multicolumn{2}{c}{ Conditions } \\
\cline { 2 - 3 } Description & heating & cooling \\
\hline B1 & not $(B 2$ or $B 3)$ & $T_{I D A}>25$ or $T_{I D A}-T_{I D A}^{\text {set }}>1$ \\
B2 & $T_{O D A}<-10$ & $s_{\text {comp }}$ is 1 \\
B3 & $T_{I D A}-T_{I D A}^{\text {set }}>1$ & $\operatorname{not~}(B 2$ or $B 3)$ \\
B4 & $T_{O D A}<-10$ & $s_{\text {comp }}$ is 1 \\
B5 & $T_{I D A}-T_{I D A}^{\text {set }}>3$ & $T_{I D A}>25$ or $T_{I D A}-T_{I D A}^{\text {set }}>3$ \\
B6 & $T_{I D A}-T_{I D A}^{\text {set }} \leq-1$ & $T_{I D A} \leq 25$ or $T_{I D A}-T_{I D A}^{\text {set }} \leq-1$ \\
B7 & $T_{I D A}-T_{I D A}^{\text {set }}>3$ & $T_{I D A}>25$ or $T_{I D A}-T_{I D A}^{\text {TSt }}>3$ \\
B8 & $T_{O D A} \geq-10$ & $s_{\text {comp }}$ is 2 \\
B9 & $T_{O D A}<-10$ & $s_{\text {comp }}$ is 1 \\
\hline
\end{tabular}




\section{A.2. HVAC parameters}

Section 3.3.1 describes how to calculate the electric heater. Equation (3.29) allows calculating the dissipated heat flow [101]. Table A.6 lists the necessary parameters. This section also lists further parameters for the CRM that is described in Section 3.3.2.

Table A.6. - Parameters of the electric heater (Heater $=\operatorname{struct}() ;)$

\begin{tabular}{ccc}
\hline Parameters & Value & Description \\
\hline$d_{1}$ & $0.0085 \mathrm{~m}$ & Diameter of the heating bar \\
$d_{2}$ & $0.002 \mathrm{~m}$ & Diameter of the heating bar incl. fins \\
$s_{1}$ & $0.041 \mathrm{~m}$ & Distance between heating bars \\
$s_{2}$ & $0.032 \mathrm{~m}$ & Distance between heating bar rows \\
$s_{3}$ & $0.002 \mathrm{~m}$ & Fin thickness \\
$n_{a}$ & 6 & Number of heating bars per row \\
$n_{b}$ & 5 & Number of heating bar rows \\
$n_{c}$ & 120 & Number of fins per bar \\
$n_{1}$ & 10 & Number of bars of the $1^{\text {st }}$ stage \\
$n_{2}$ & 20 & Number of bars of the $2^{\text {nd }}$ stage \\
$t_{r}$ & $0.003 \mathrm{~m}$ & Distance between fins \\
$l_{\text {length }}$ & $0.650 \mathrm{~m}$ & Length of a heating bar \\
$l_{\text {width }}$ & $0.275 \mathrm{~m}$ & Width of the electric heater \\
fluchtend & true & Aligned arrangement \\
$\lambda_{\text {Ri }}$ & $200 \mathrm{~W} /(\mathrm{m} \mathrm{K})$ & thermal conductivity coefficient of fins \\
$\lambda_{S t}$ & $330 \mathrm{~W} /(\mathrm{m} \mathrm{K})$ & thermal conductivity coefficient of heating bars \\
$R_{\text {Stab0 }}$ & $490.8 \Omega$ & electrical resistance of heating bars \\
$\eta_{1}$ & 0.7 & Efficiency of the $1^{\text {st }}$ stage \\
$\eta_{2}$ & $3 \mathrm{~kJ} / \mathrm{K}$ & Efficiency of the $2^{\text {nd }}$ stage \\
$C_{1}$ & $6 \mathrm{~kJ} / \mathrm{K}$ & Heat capacity of the $1^{\text {st }}$ stage \\
$C_{2}$ & & Heat capacity of the $2^{\text {nd }}$ stage \\
\hline & &
\end{tabular}


Table A.7. - Parameters of the cooling cycle (CoolingCycle. = struct ();)

\begin{tabular}{|c|c|c|}
\hline Parameters & Value & Description \\
\hline$T_{\text {subcooled }}$ & $2^{\circ} \mathrm{C}$ & subcooled temperature \\
\hline$T_{\text {overheated }}$ & $10^{\circ} \mathrm{C}$ & overheating temperature \\
\hline$\eta_{s v}$ & 0.4563 & Compressor efficiency in the operating point \\
\hline$\dot{m}_{K M, k o r r 1}$ & $0.5845 \mathrm{~m}^{3} / \mathrm{s}$ & Correcting summand with Bypass \\
\hline$\dot{m}_{K M, k o r r 2}$ & $0.7028 \mathrm{~m}^{3} / \mathrm{s}$ & Correcting summand without bypass \\
\hline$d_{a}$ & $0.00921 \mathrm{~m}$ & Outer diameter of the pipe \\
\hline$d_{i}$ & $0.0085 \mathrm{~m}$ & Inner diameter of the pipe \\
\hline$s_{1}$ & $0.02829 \mathrm{~m}$ & $\begin{array}{l}\text { Distance between two pipes perpendicular to } \\
\text { flow direction (Longitudinal splitting) }\end{array}$ \\
\hline$s_{2}$ & $0.01353 \mathrm{~m}$ & $\begin{array}{l}\text { Distance between to pipes in } \\
\text { flow direction (Horizontal splitting) }\end{array}$ \\
\hline$s_{3}$ & $0.0001 \mathrm{~m}$ & fin thickness \\
\hline$t_{r}$ & $0.00285 \mathrm{~m}$ & Distance between fins \\
\hline$n_{a}$ & 8 & Numbers of pipes per pipe row \\
\hline$n_{b}$ & 5 & Number of pipe rows \\
\hline$n_{c}$ & 238 & Number of fines per pipe \\
\hline$l_{\text {length }}$ & $0.700 \mathrm{~m}$ & Length of the heat exchanger (pipe length) \\
\hline$l_{\text {width }}$ & $0.300 \mathrm{~m}$ & Width of the heat exchanger \\
\hline$l_{\text {depth }}$ & $0.108 \mathrm{~m}$ & Depth of the heat exchanger \\
\hline fluchtend & False & Aligned arrangement \\
\hline$\lambda_{R i}$ & $200 \mathrm{~W} /(\mathrm{m} \mathrm{K})$ & Distance between heating bars \\
\hline$\lambda_{S t}$ & $330 \mathrm{~W} /(\mathrm{m} \mathrm{K})$ & Distance between heating bars \\
\hline
\end{tabular}




\section{A.3. Parameters of the tram}

The heat transfer coefficient was calculated for individual parts of the Ultra Low Floor (ULF).

Table A.8. - Calculated heat transfer coefficient for individual tram parts as a function of the tram speed

\begin{tabular}{lrrrr}
\hline \multirow{2}{*}{ Description } & \multicolumn{4}{c}{ Speed in $\mathrm{km} / \mathrm{h}$} \\
\cline { 2 - 5 } & 0 & 12 & 50 & 80 \\
\hline Bow & 2.73 & 3.63 & 4.39 & 4.54 \\
Portal bow and tail & 1.77 & 2.23 & 2.61 & 2.68 \\
Middle section bow and tail & 2.41 & 3.16 & 3.79 & 3.92 \\
Joint portal & 2.67 & 3.54 & 4.28 & 4.43 \\
Middle section & 2.36 & 3.09 & 3.72 & 3.84 \\
Joint portal & 2.67 & 3.54 & 4.28 & 4.43 \\
Middle section bow and tail & 2.41 & 3.16 & 3.79 & 3.92 \\
Portal bow and tail & 1.77 & 2.23 & 2.61 & 2.68 \\
Tail & 2.44 & 3.21 & 3.87 & 4 \\
\hline
\end{tabular}




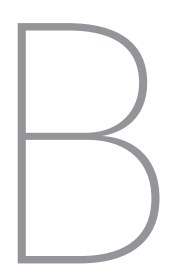

\section{More results}

\section{B.1. Daily report}

This section shows an example of a daily report. The original daily reports are in the project language (German). Some of the daily reports are summarised in [50].

\section{B.1.1. Environmental conditions}

In environmental conditions, all (measured) variables are summarised, which affect the electrical power consumption of the HVAC and the thermal comfort of passengers in the tram during a measurement day.

\section{Weather}

The weather is one of the major effects on the power consumption of the HVAC. The shown data are used as comparison and addition for the measurement data [103].
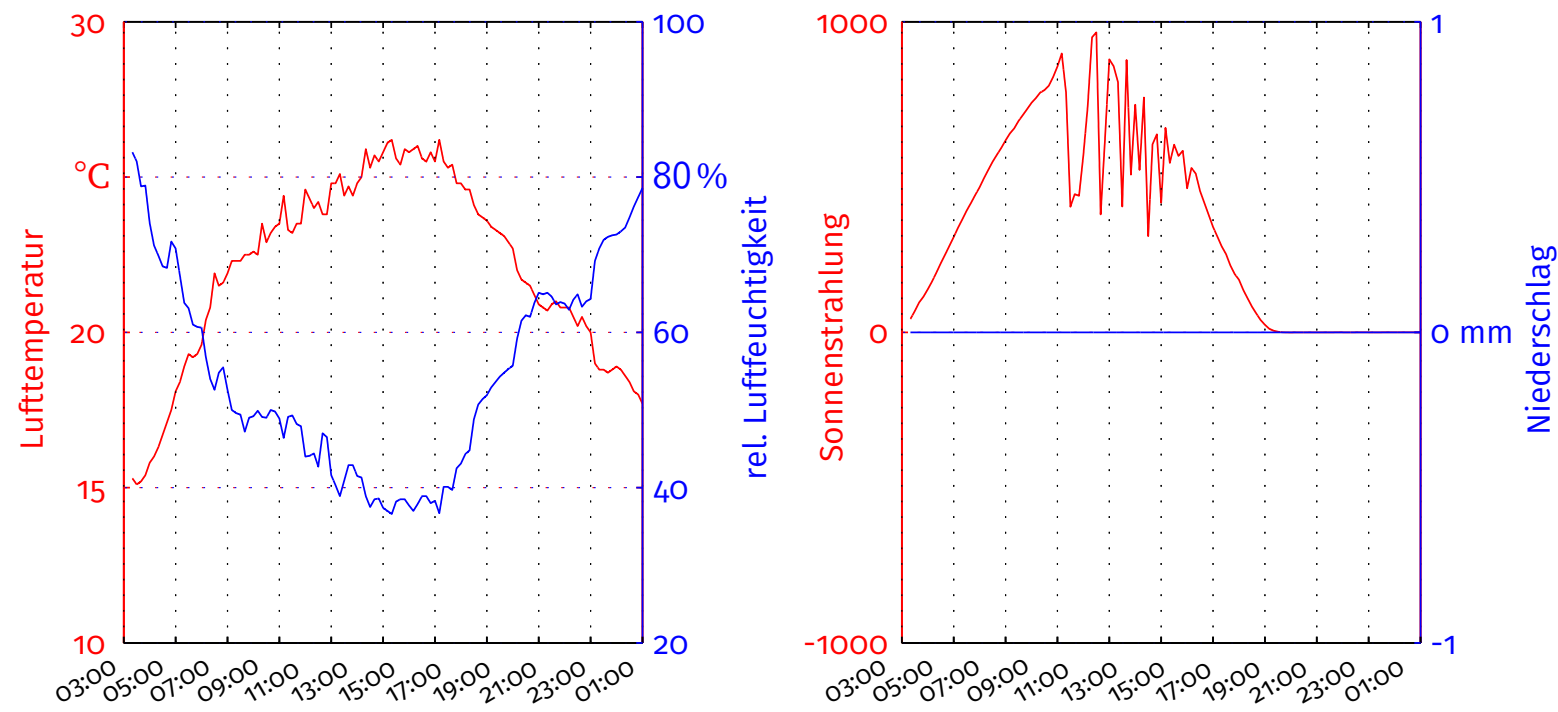

Figure B.1. - left: conditions of the outdoor air (temperature and relative humidity), right: solar radiation and rain 


\section{Travelled route}

The travelled route has another major effect on the power consumption of the HVAC. The tram mainly drove on line 62 .

City map The distance travelled by the tram was printed onto a city map [92].

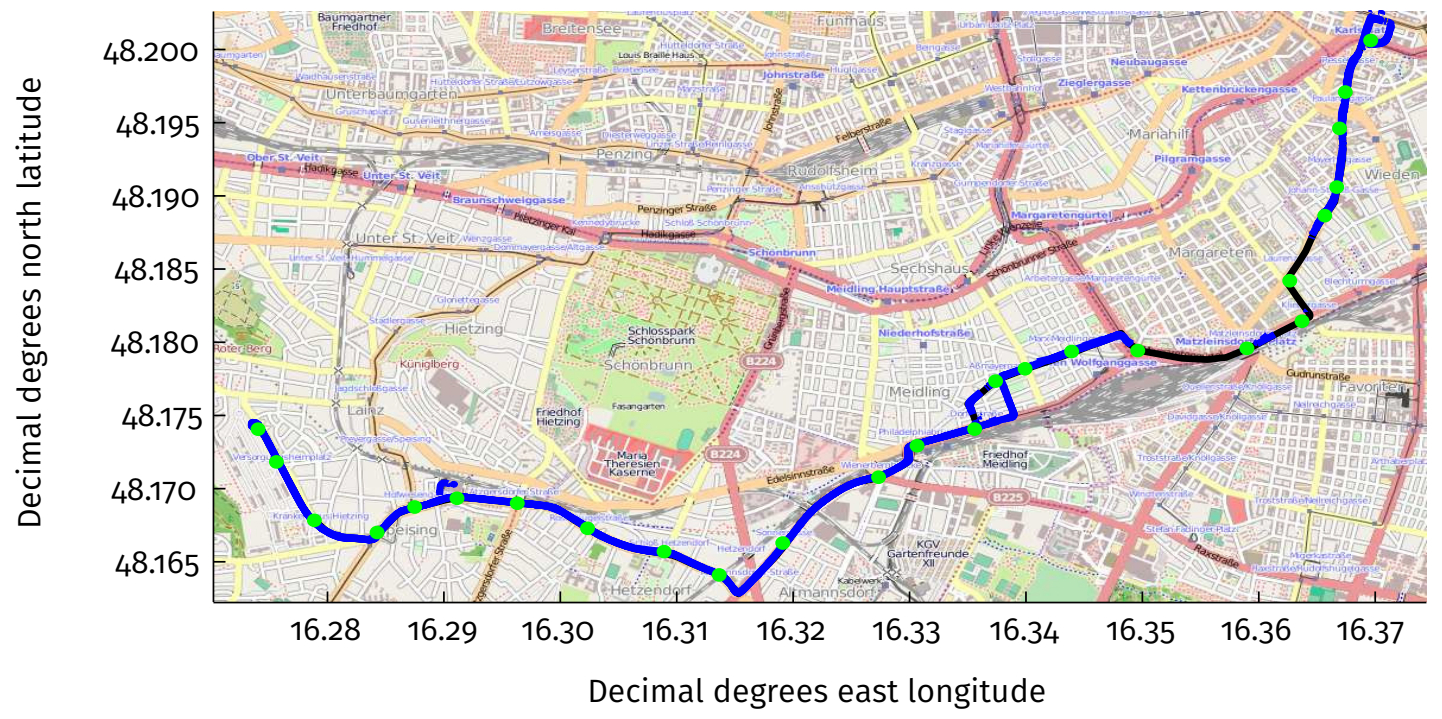

Figure B.2. - Travelled Route, + Route of the tram (line 62), - travelled route of the tram, $\bullet$ stops

Distance-time graph The following figure shows the distance of the tram to the $1^{\text {st }}$ stop. The stations of line 62 (out of town) are plotted on the y-axis. Therefore, the location of the tram is specified during the test time.

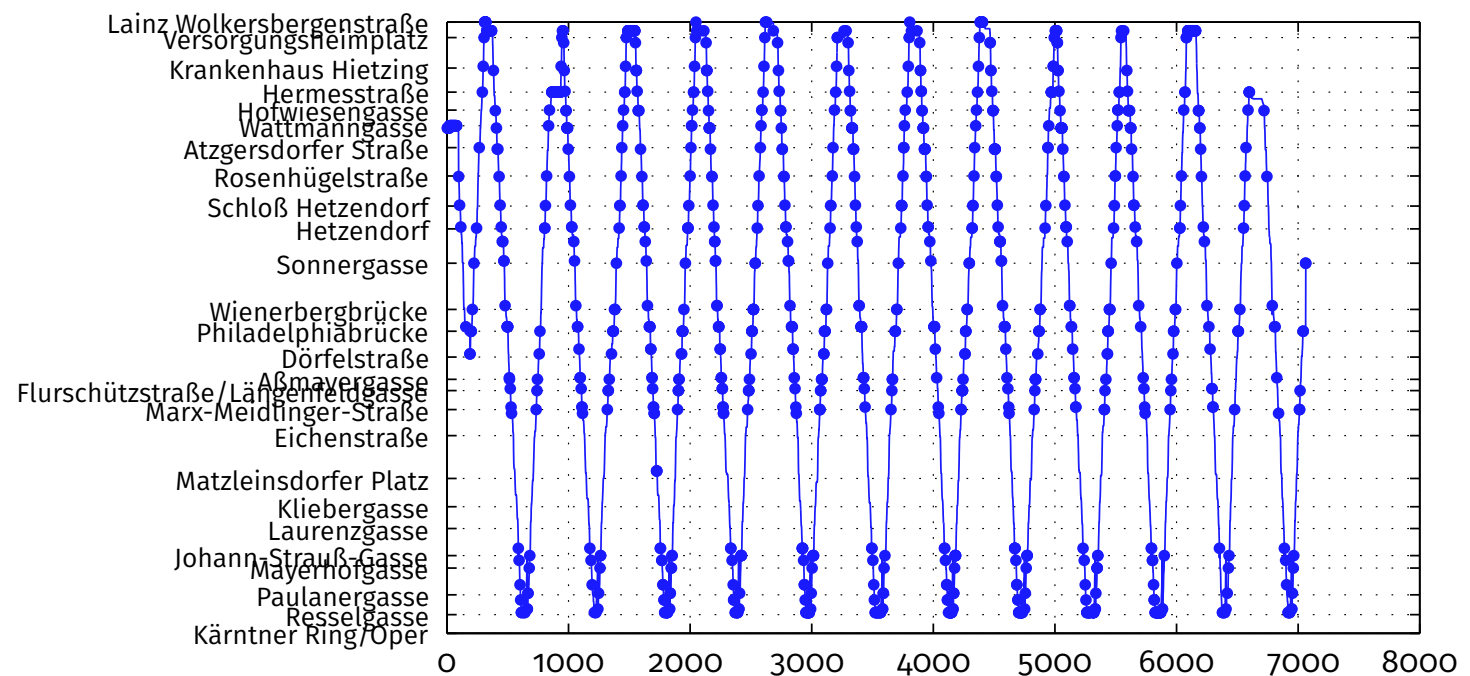

Figure B.3. - Distance-time graph shows the location of the tram for the test time. Stops are marked as •. 
Many variables have an effect in the tram. The following environmental conditions were measured

- the outdoor air temperature,

- the solar radiation on the tram, and

- the number of passengers.

The conditions are shown for every section ( $1-3)$ separately.

Outdoor air temperature The ODA of the tram was measured in the incoming air [95].
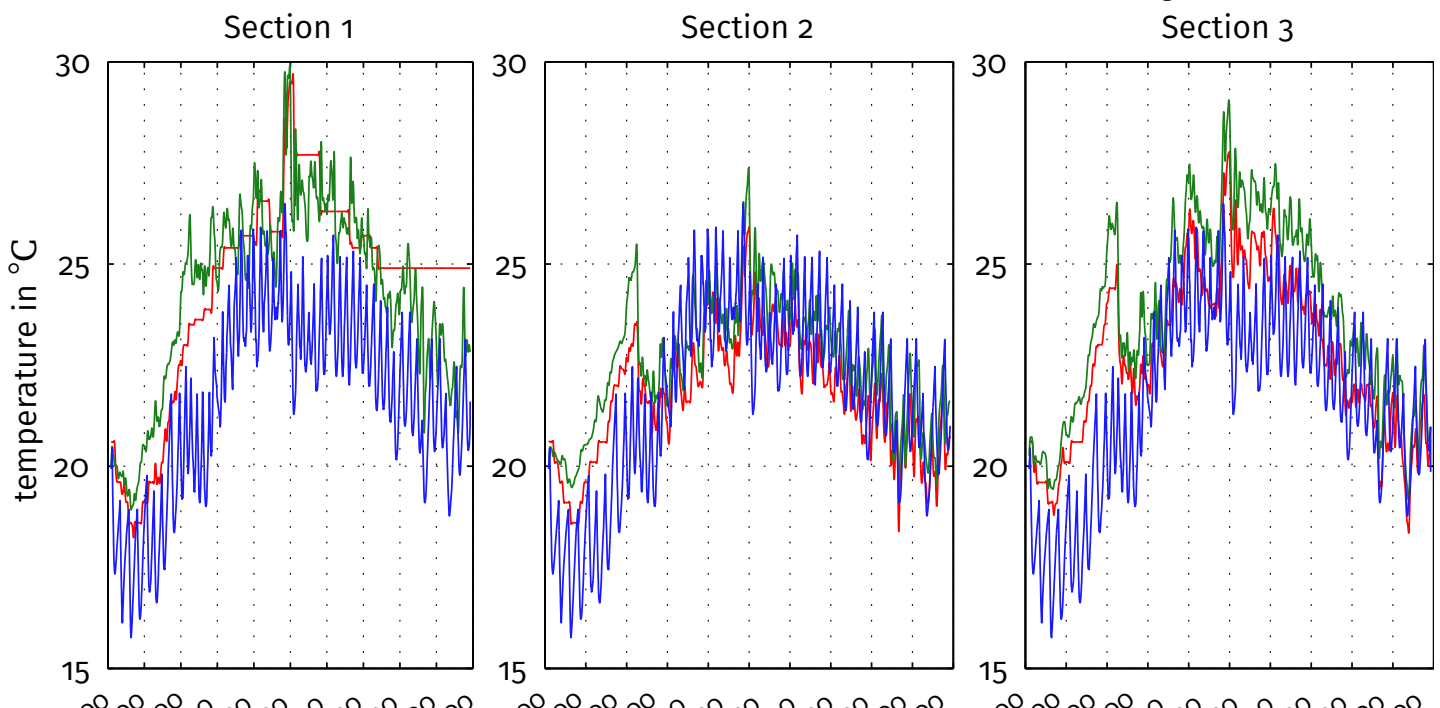

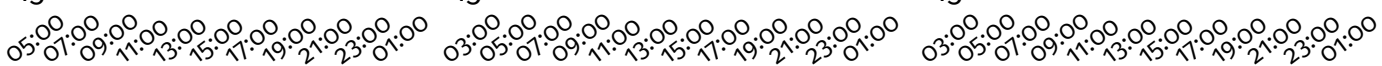

Figure B.4. - Outdoor air temperature for every section $(1-3)$ of the tram

Solar radiation on to the tram The solar radiation on to the (measurement by Rail Tec Arsenal Fahrzeugversuchsanlage GmbH (RTA)) the tram is already given as heat flow. The calculation is done according to Equation (3.12).
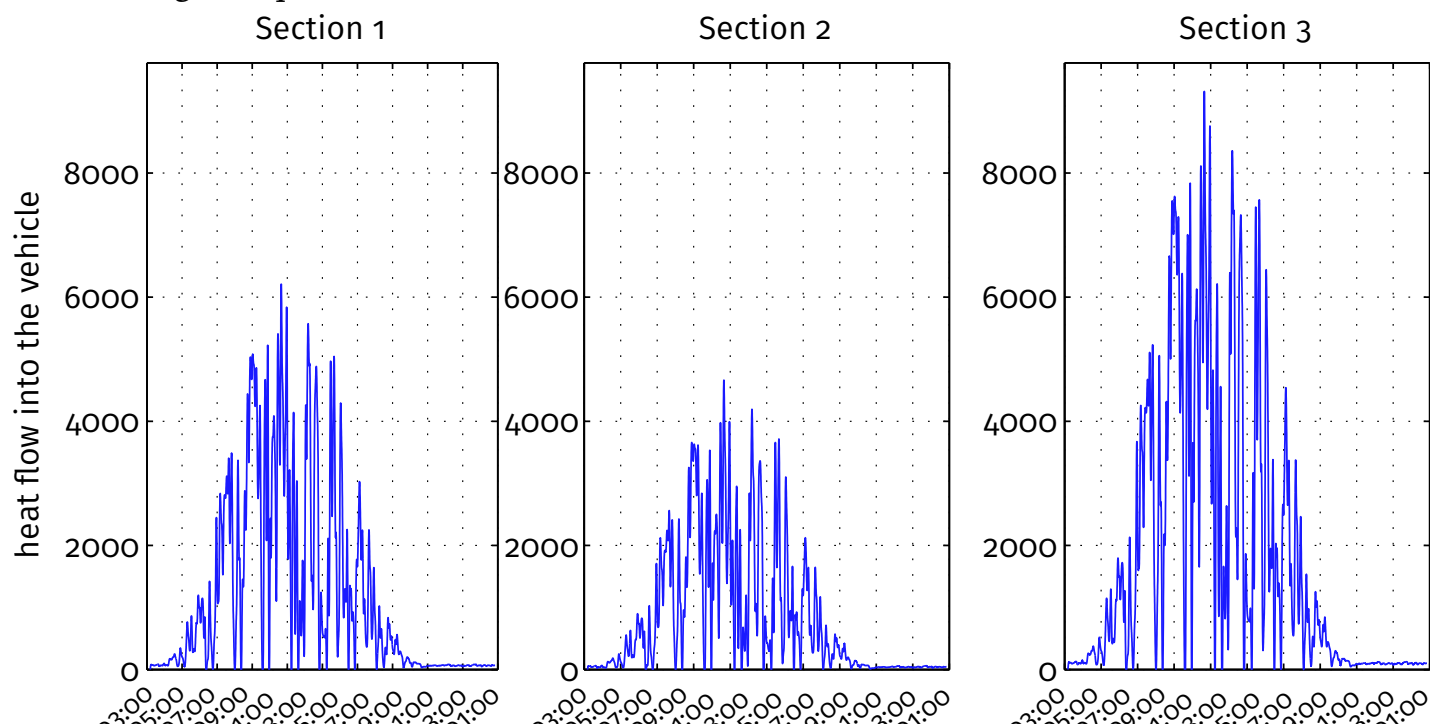

Figure B.5. - Solar radiation onto the vehicle for every section (1-3) of the tram 
Number of passengers The load signal of the tram (measurement by Siemens AG Österreich (SAGOE)) was converted to a number of persons inside the tram.
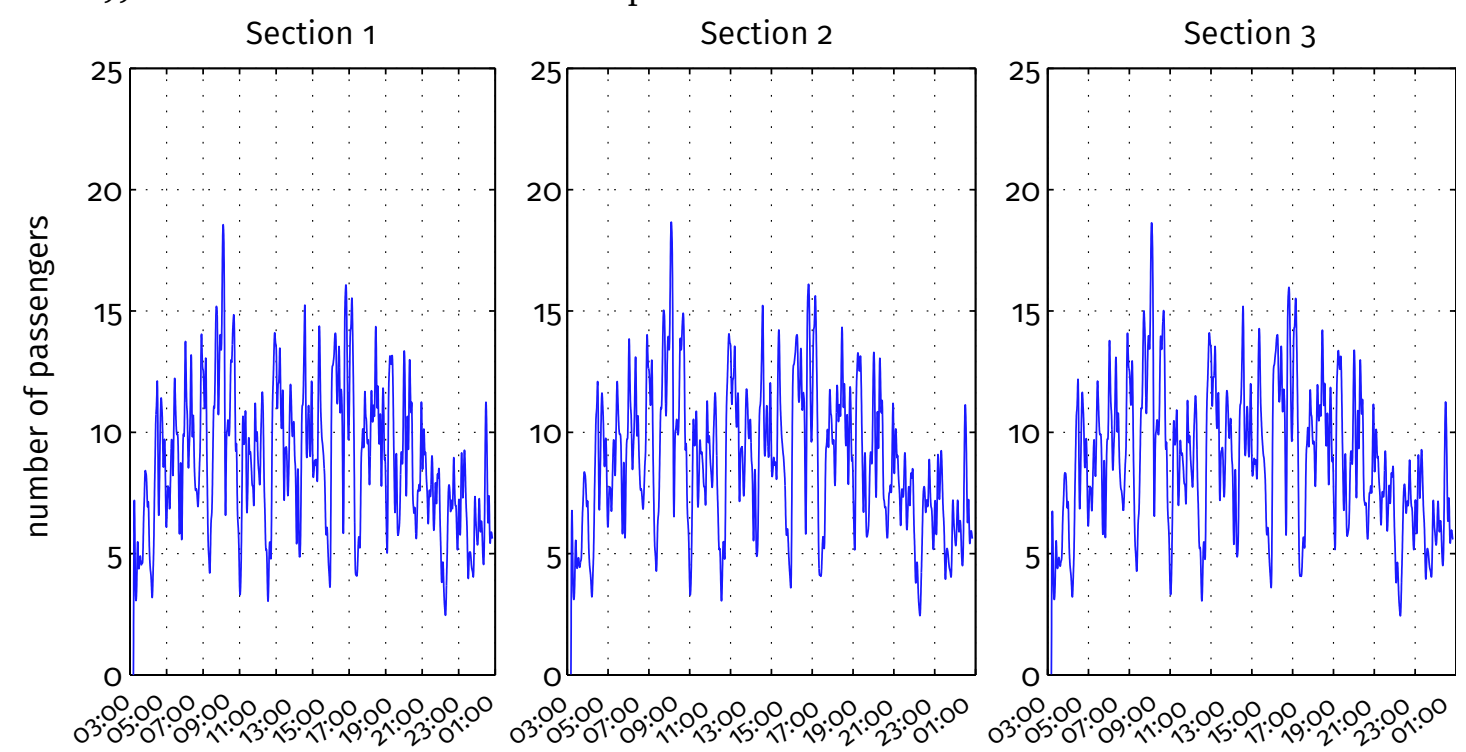

Figure B.6. - Number of Passengers for every section (1-3) of the tram

\section{B.1.2. Tram}

Conditions of the indoor air

The conditions of the indoor air can be clearly described by two variables (and the air pressure). The temperature and the relative humidity are specified.

Indoor air temperature With the inputs given above the indoor air temperature was calculated for every section of the tram (see Equation (3.1)).
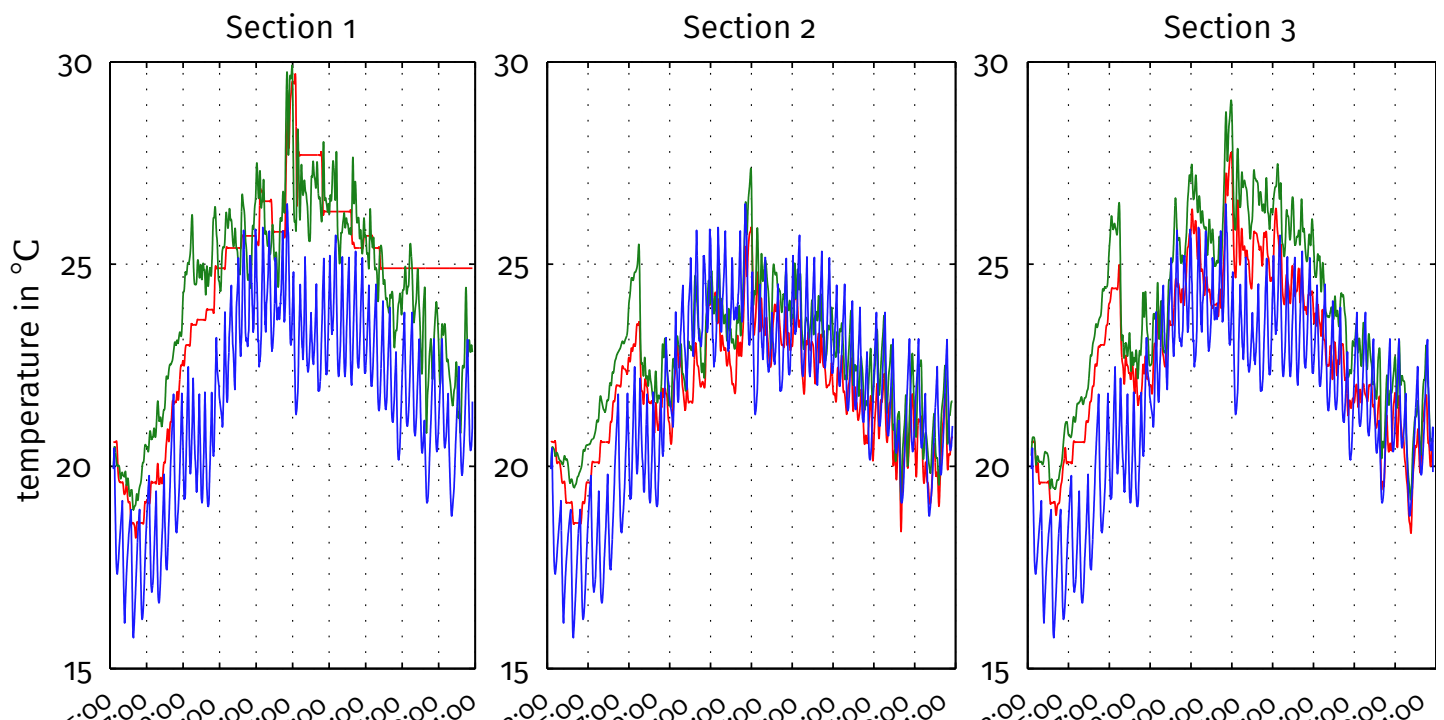

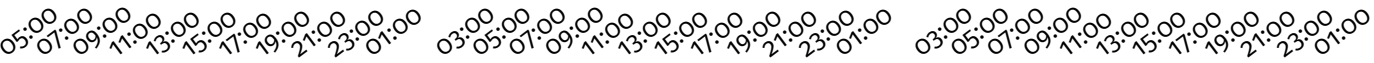
Figure B.7. - Indoor air temperature in every section(1-3) of the tram 
Relative humidity of the indoor air With the inputs given above, the humidity of the indoor air was calculated for every section of the tram (see Chapter 3 ).

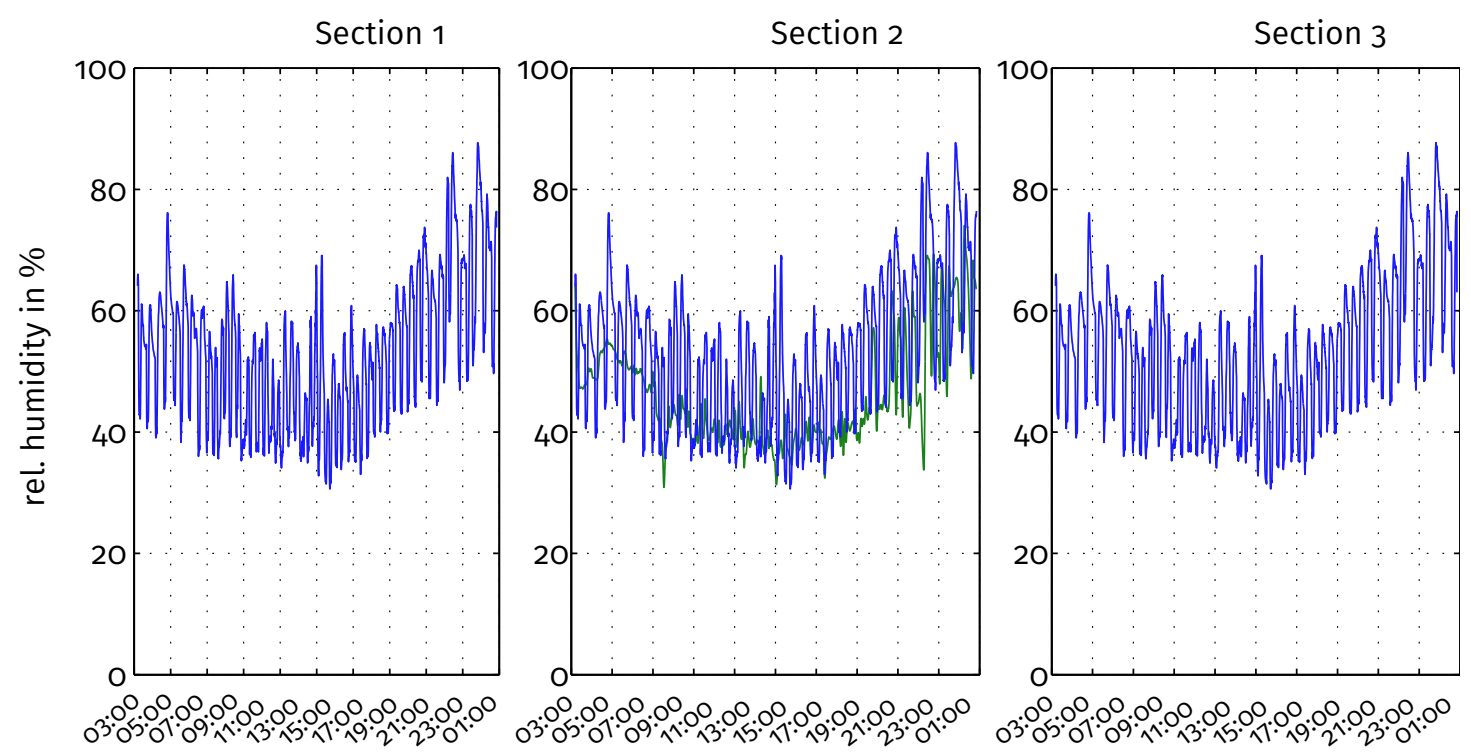

Figure B.8. - Relative humidity of the indoor air for every section(1-3) of the tram

\section{Thermal comfort}

Predicted mean vote (PMV) The predicted mean vote was calculated from the temperature and the relative humidity of the indoor air as well as the outdoor air temperature[61]. The desired range $(-0.5 \leq$ PMV $\leq 0.5)$ is highlighted in green.
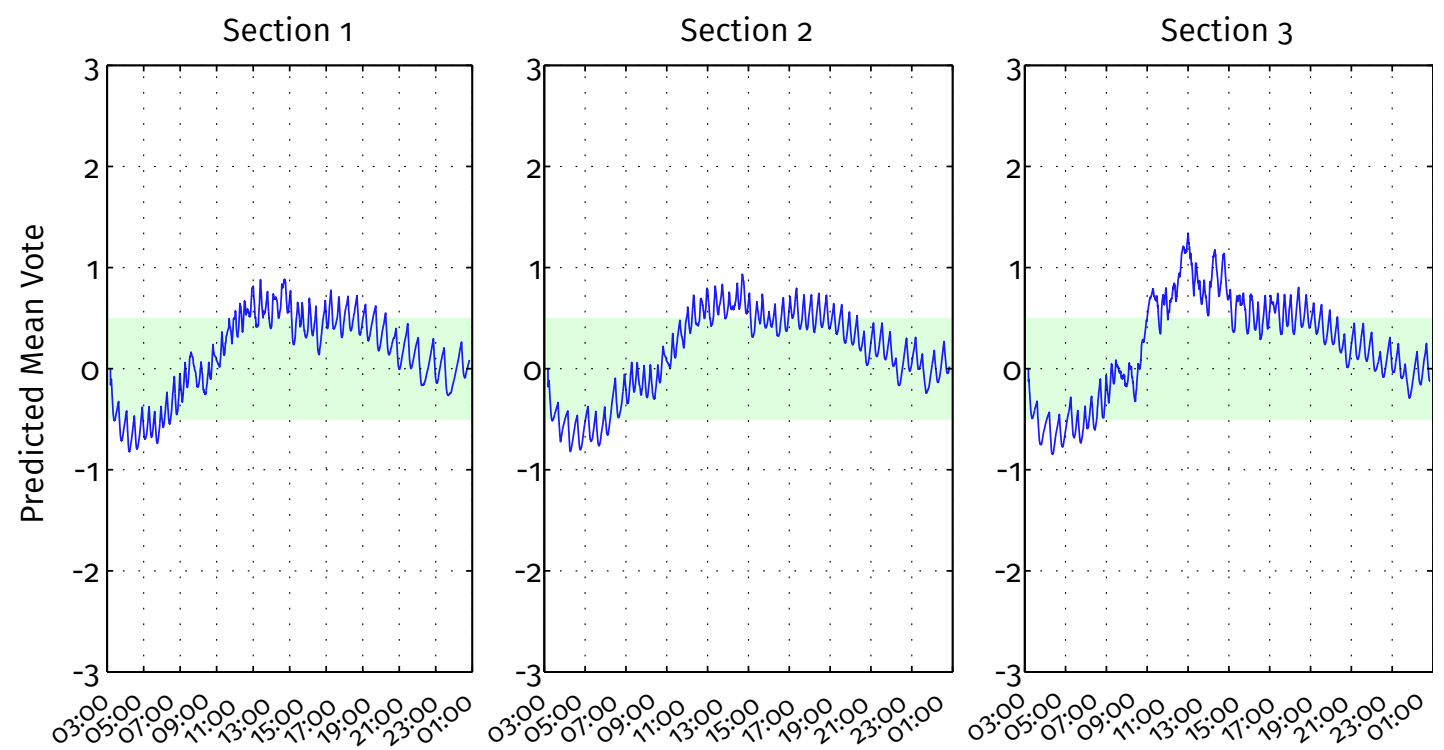

Figure B.9. - Thermal comfort given as predicted mean vote 
Percentage persons dissatisfied (PPD) The given percentage of dissatisfied persons refers to the persons that are dissatisfied with the thermal comfort. This value follows from the PMV [61].

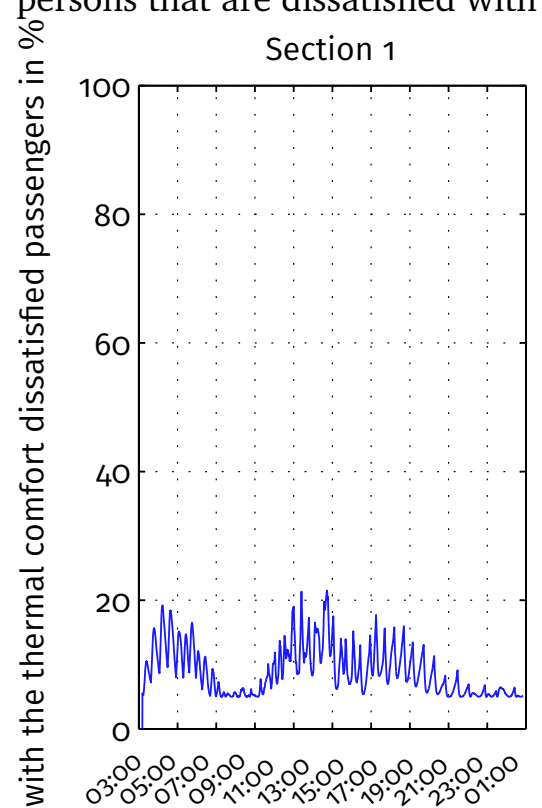

Section 2

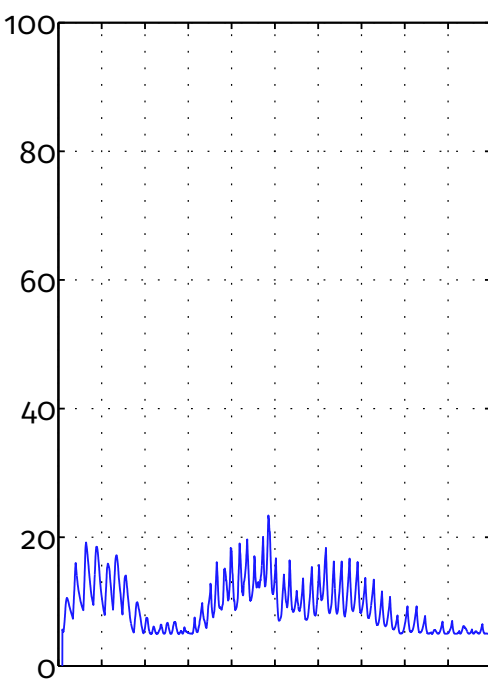

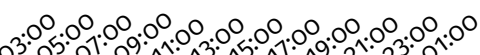
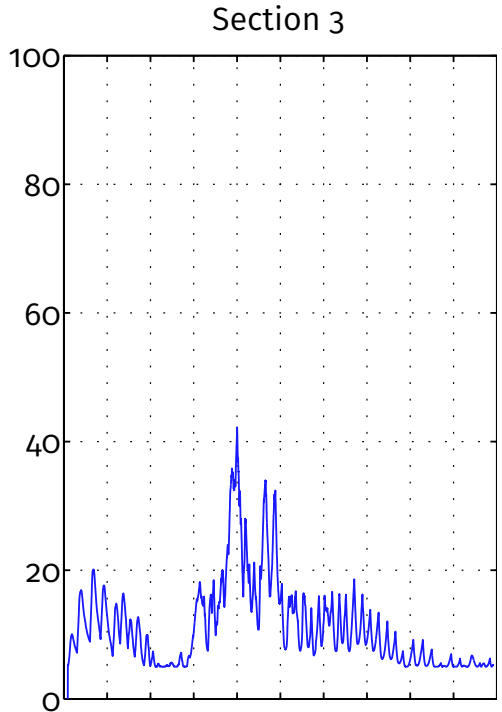

(1) Figure B.10. - The percentage shown refers to, with the thermal comfort, dissatisfied passengers.

\section{B.1.3. HVAC}

The most important informations about the HVAC are the condition of the supply air and the energy consumption. They are shown in detail.

\section{Condition of the supply air}

Again, the condition of the supply air is specified by two variables (and the air pressure). Therefore, the temperature and the relative humidity are shown.
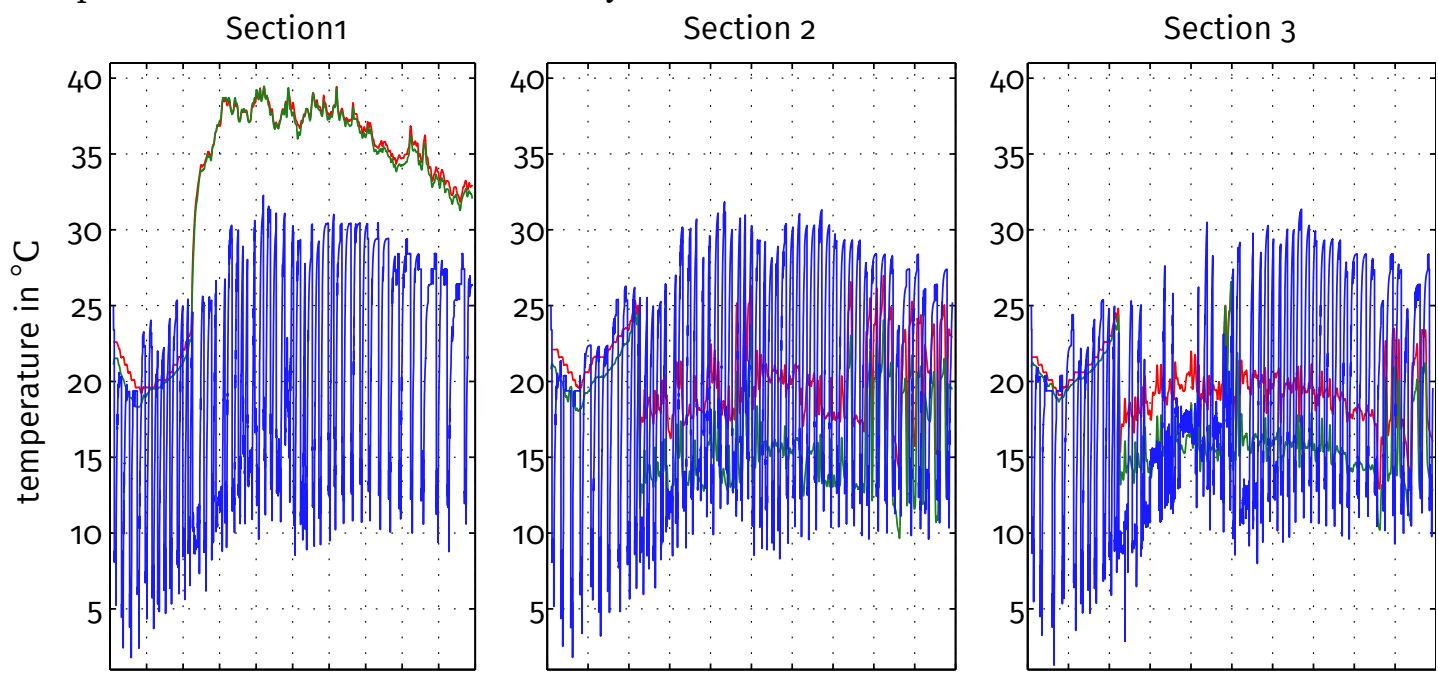

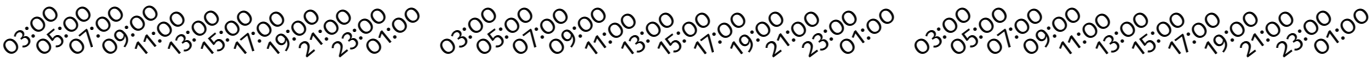
Figure B.11. - Temperature of the supply air for every section(1-3) of the tram 

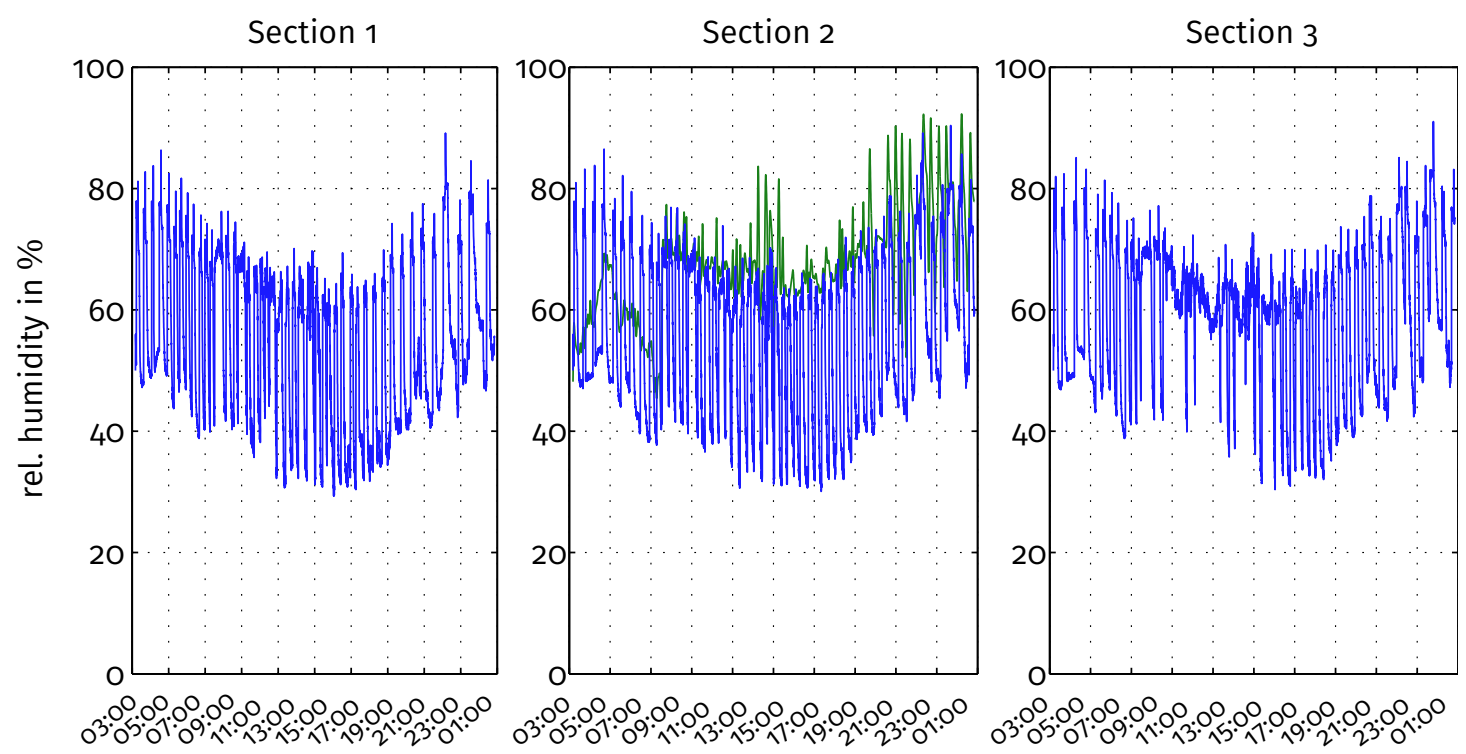

Figure B.12. - Relative humidity of the supply air for every section(1-3) of the tram

\section{Power consumption}

There are two voltage levels ( $400 \mathrm{~V}$ and $600 \mathrm{~V}$ ) in the HVAC. They are shown separately. In addition, the difference between the measured and the simulated power consumption are depicted.

$400 \mathrm{~V}$ power consumption The $400 \mathrm{~V}$ consumer are the supply air fan of the HVAC, the compressor, and the cooling air fan of the CRM.
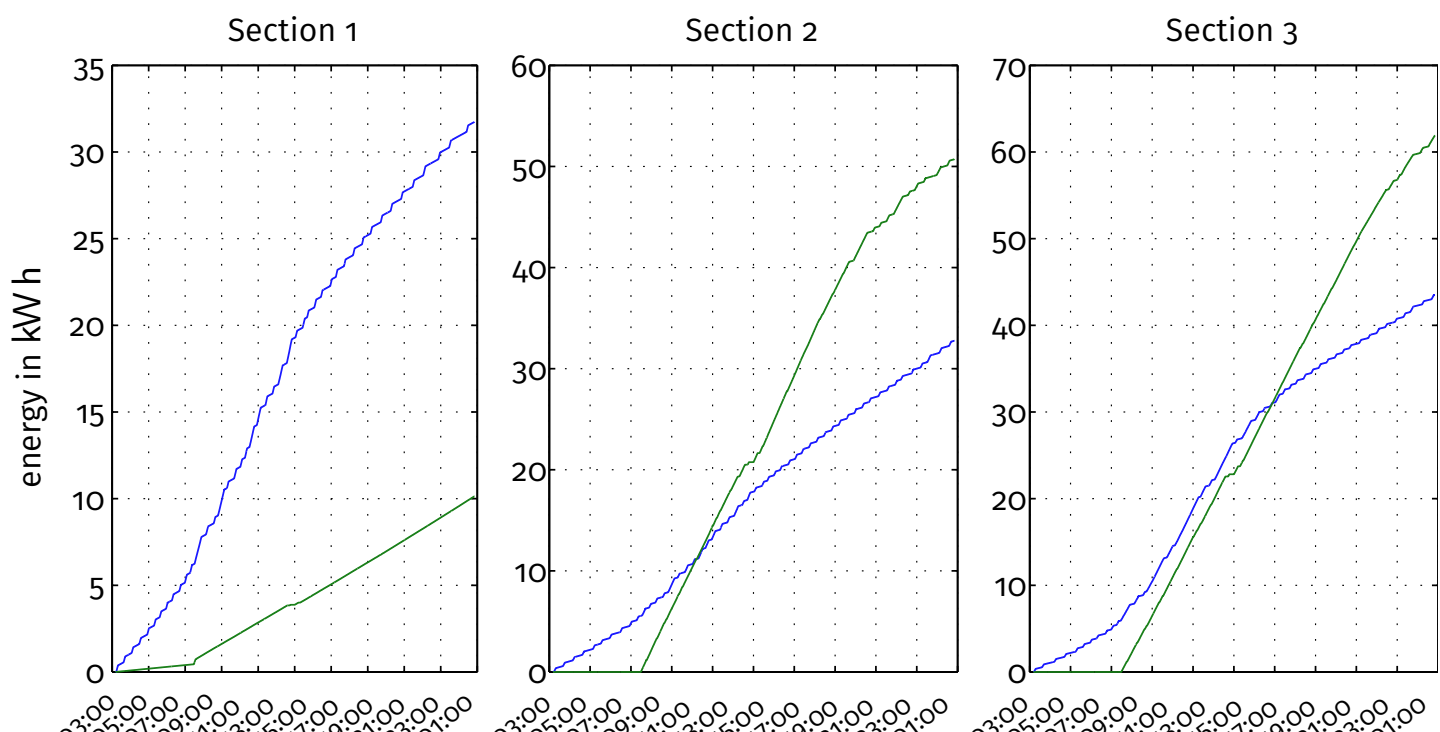

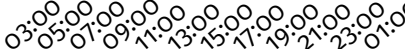

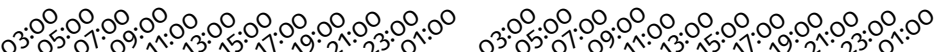

Figure B.13. - Energy consumption of the $400 \mathrm{~V}$ consumer for every section(1-3) of the tram 
$600 \mathrm{~V}$ power consumption The only $600 \mathrm{~V}$ consumer of the HVAC is the electric heater.
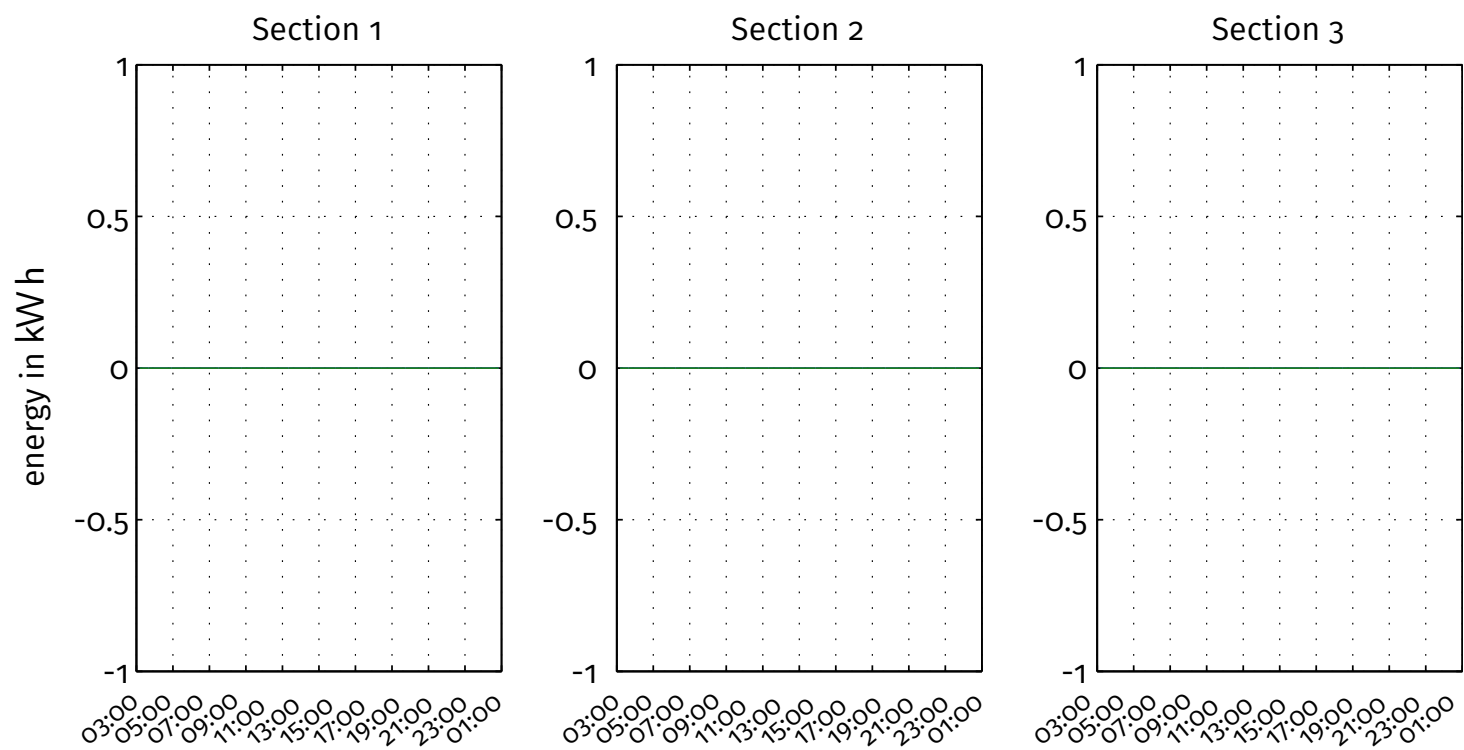

Figure B.14. - Energy consumption of the $600 \mathrm{~V}$ consumer for every section(1-3) of the tram

Comparison between simulation and measurement Comparison of the power consumption for all HVACs for the given time.

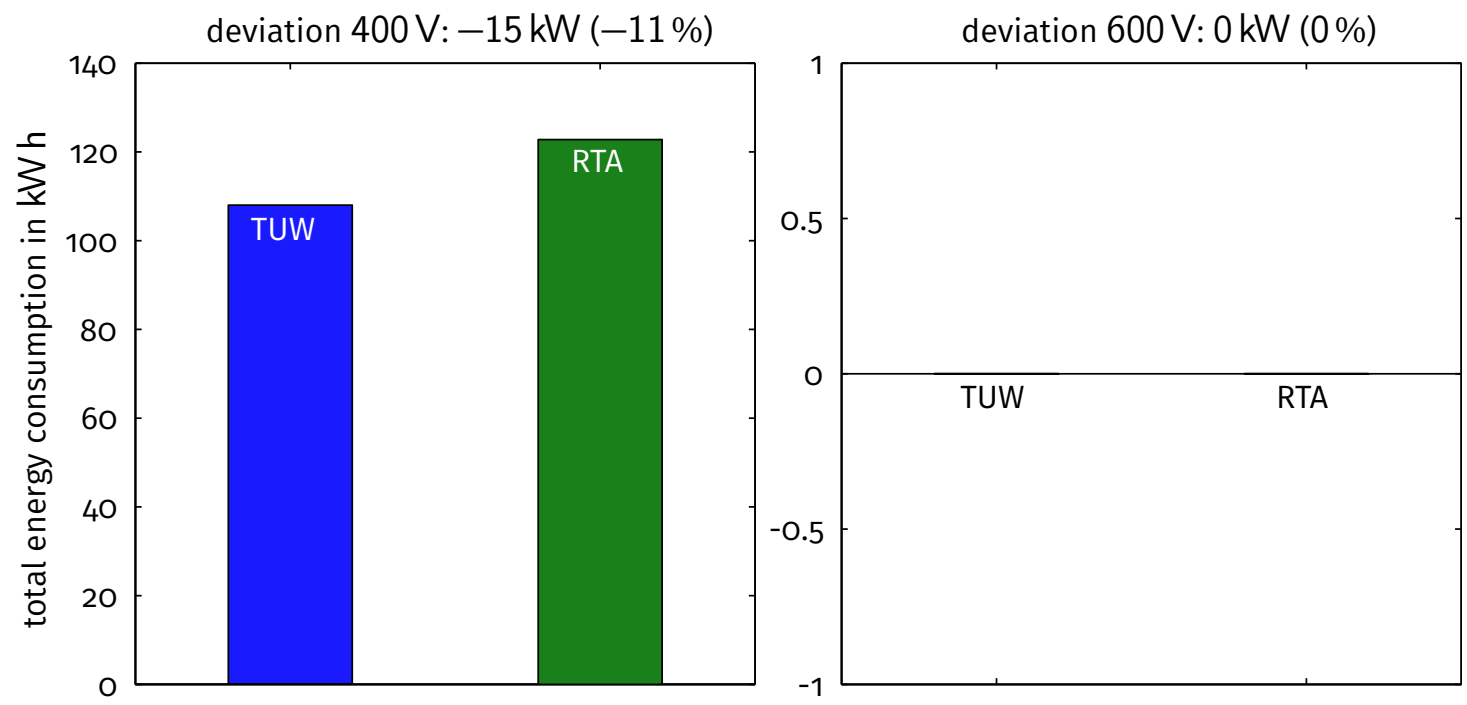

Figure B.15. - Comparison between simulation and measurement: left: $400 \mathrm{~V}$ consumer, right: $600 \mathrm{~V}$ consumer. 


\section{Control}

The state of the controller is calculated from the influences of the environment and the conditions of the indoor air temperature. The most important state is the global state (Heating/Cooling) of the controller.

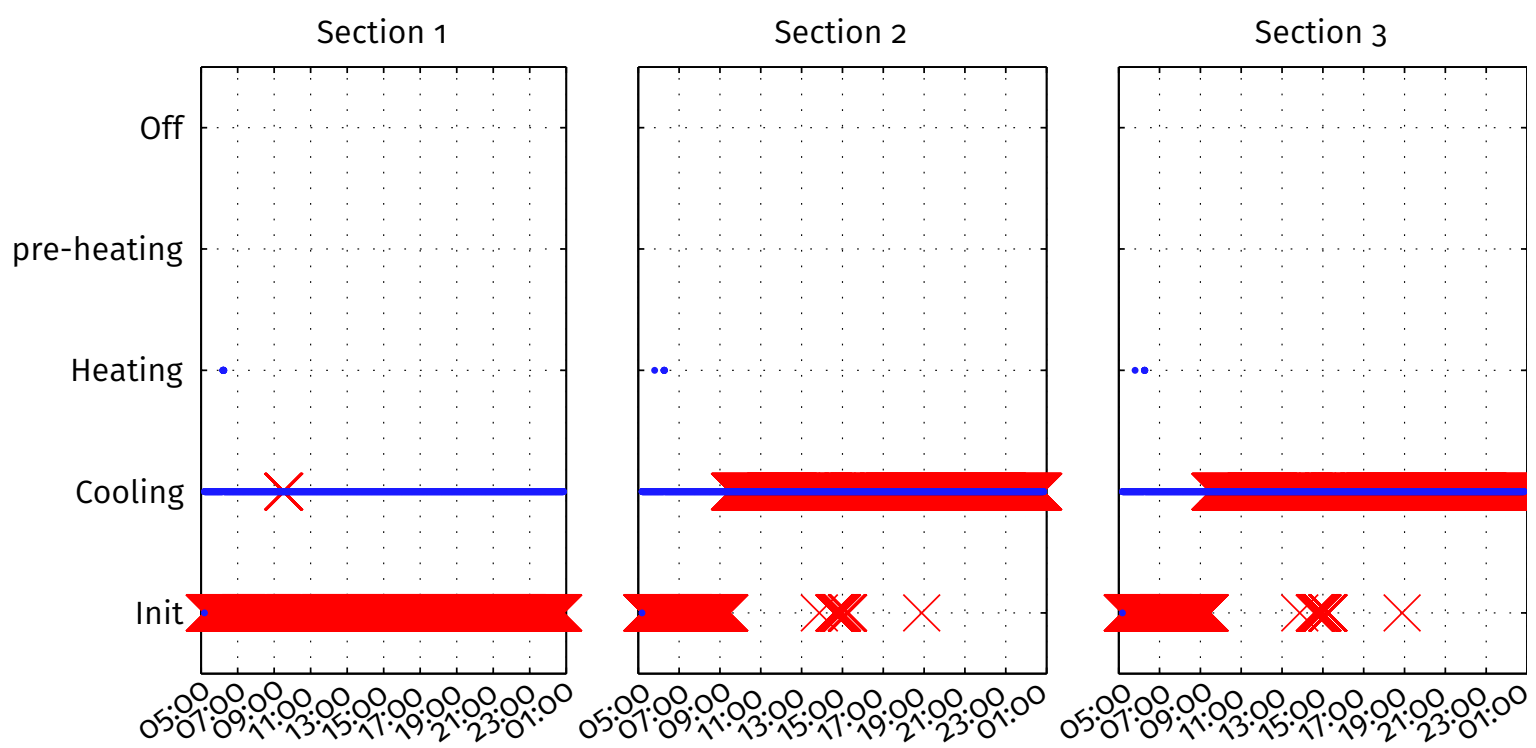

Figure B.16. - global state of the controller for every section(1-3) of the tram

\section{B.2. Performance maps of the CRM}

The proposed control algorithm needs to know the supplied respectively absorbed heat by the CRM as accurately as possible. It was not possible to rely on empirical values or calculation modes because of the implemented innovations. Therefore, the supplied respectively absorbed heat had to be measured.For this purpose, measurements were used which were conducted in the frame of function and performance tests of the HVAC by Vossloh Kiepe Austria GmbH (VKA) (Source 1). The primary objective of the measurements was to verify the functionality of the CRM at design conditions. As no previous knowledge was available it was particularly important that the measurements of the performance map cover the whole operating range of the heat pump and air conditioning. This was not satisfied by existing measurements, therefore additional measurements were conducted (Source 2). Measurements were conducted on two units in parallel and the average value was used for each measurement.

The output of the model is the supplied respectively absorbed heat. The model input is the temperature of the ODA, the compressor's frequency and the mass flow of the supply air fan. Table B. 3 shows the measurement data of the heat pump and Table B.5 shows the measurements of the air condition. In both cases the table also lists the measurement's source. 
The supplied respectively absorbed heat of the CRM can be calculated from

$$
\begin{gathered}
\dot{Q}=k_{1}+k_{2} T_{O D A}+k_{3} \phi_{c o m p}+k_{4} \dot{m}_{S U P} \cdots \\
+k_{5} T_{O D A}^{2}+k_{6} T_{O D A} \varphi_{c o m p}+k_{7} \varphi_{c o m p}^{2}+k_{8} \varphi_{c o m p} \dot{m}_{S U P}
\end{gathered}
$$

Table B.2a gives the estimated factors of the heat pump's performance map and Table B.2b the factors of the air condition's performance map are given. Factors were calculated by least-squares estimation.

Figure B.17 shows a visualisation of the heat pump's performance map and Figure B.19 shows the air condition's performance map. The heat absorption or heat dissipation is plotted against the compressor's frequency in $\mathrm{Hz}$ and the outdoor air in ${ }^{\circ} \mathrm{C}$. The plane is shown as a grid for the smallest air mass flow. Only grid elements are shown as points for all further combinations. Figure B.18 shows a comparison between measurements and performance map for the heat pump and Figure B.20 shows a comparison for the air condition. It can be seen that the error in heat pump operation is smaller than $\pm 2 \mathrm{~kW}$ and in air condition operation it is smaller than $\pm 1.5 \mathrm{~kW}$.

Table B.1. - Factors of the performance maps for the CRM

(a) in heat pump operation

\begin{tabular}{cr}
\hline Factor & Value \\
\hline$k_{1}$ & -6143.6493 \\
$k_{2}$ & 1209.3195 \\
$k_{3}$ & 89.4526 \\
$k_{4}$ & 4766.6724 \\
$k_{5}$ & -49.5705 \\
$k_{6}$ & -1.4199 \\
$k_{7}$ & -0.28205 \\
$k_{8}$ & 118.5537 \\
\hline
\end{tabular}

(b) in air condition operation

\begin{tabular}{cr}
\hline Factor & Value \\
\hline$k_{1}$ & -4031.6397 \\
$k_{2}$ & 521.2184 \\
$k_{3}$ & 47.2751 \\
$k_{4}$ & -6218.3084 \\
$k_{5}$ & -7.3183 \\
$k_{6}$ & 0.33241 \\
$k_{7}$ & -0.48299 \\
$k_{8}$ & 192.6419 \\
\hline
\end{tabular}




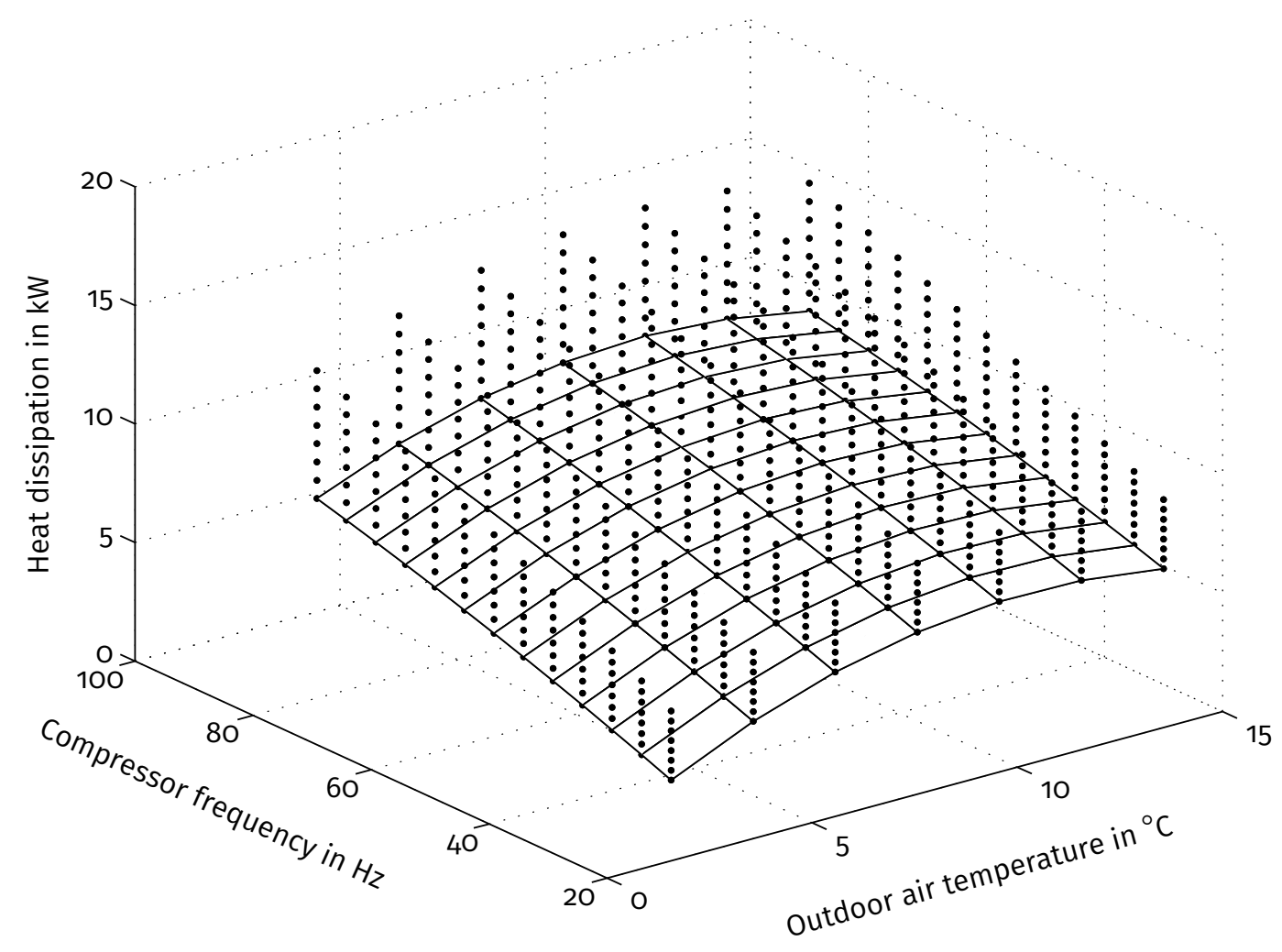

Figure B.17. - Performance Map in Heat Pump Mode 


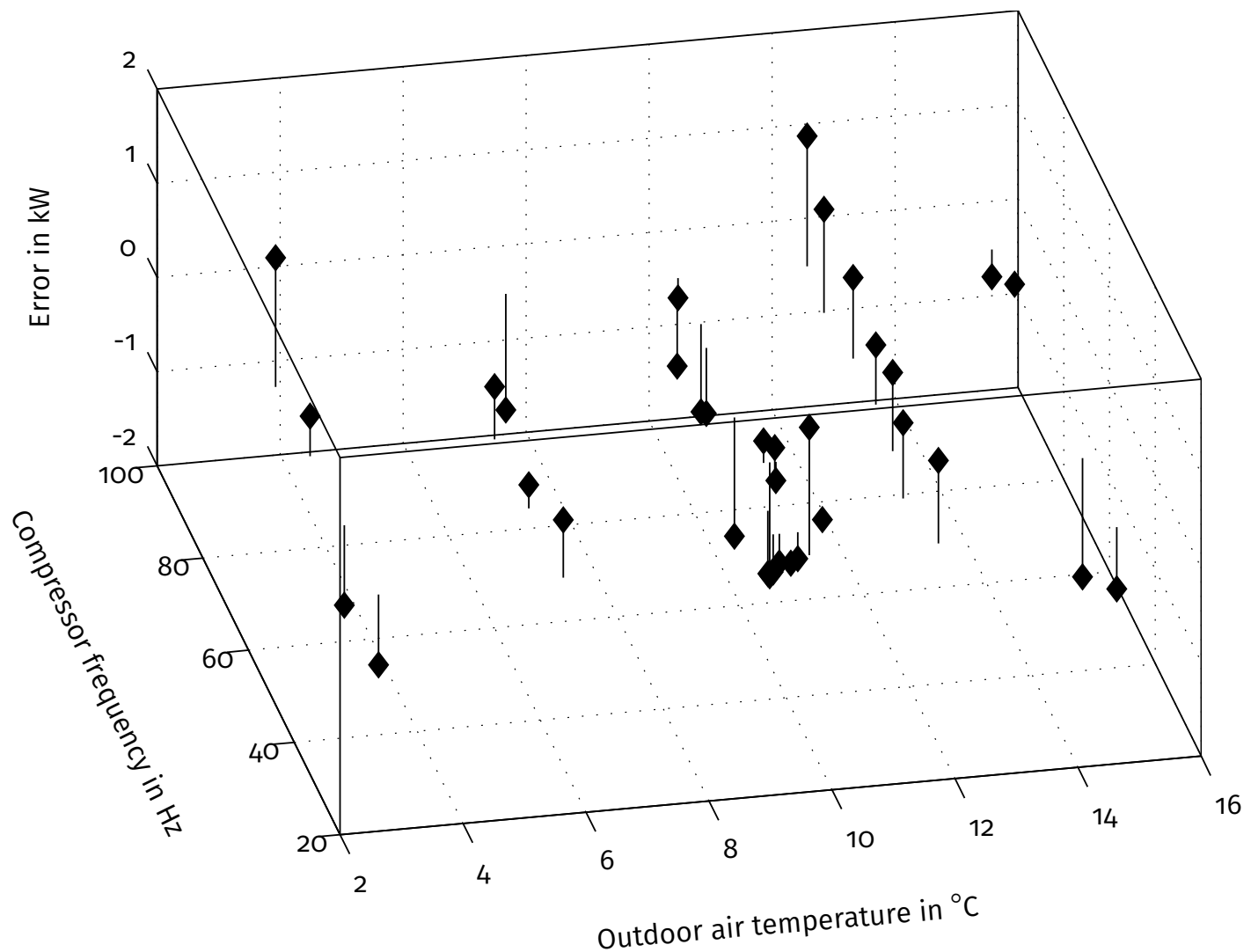

Figure B.18. - Error between HP performance map and measurement data (Q_HP_est-Q_HP_meas) 


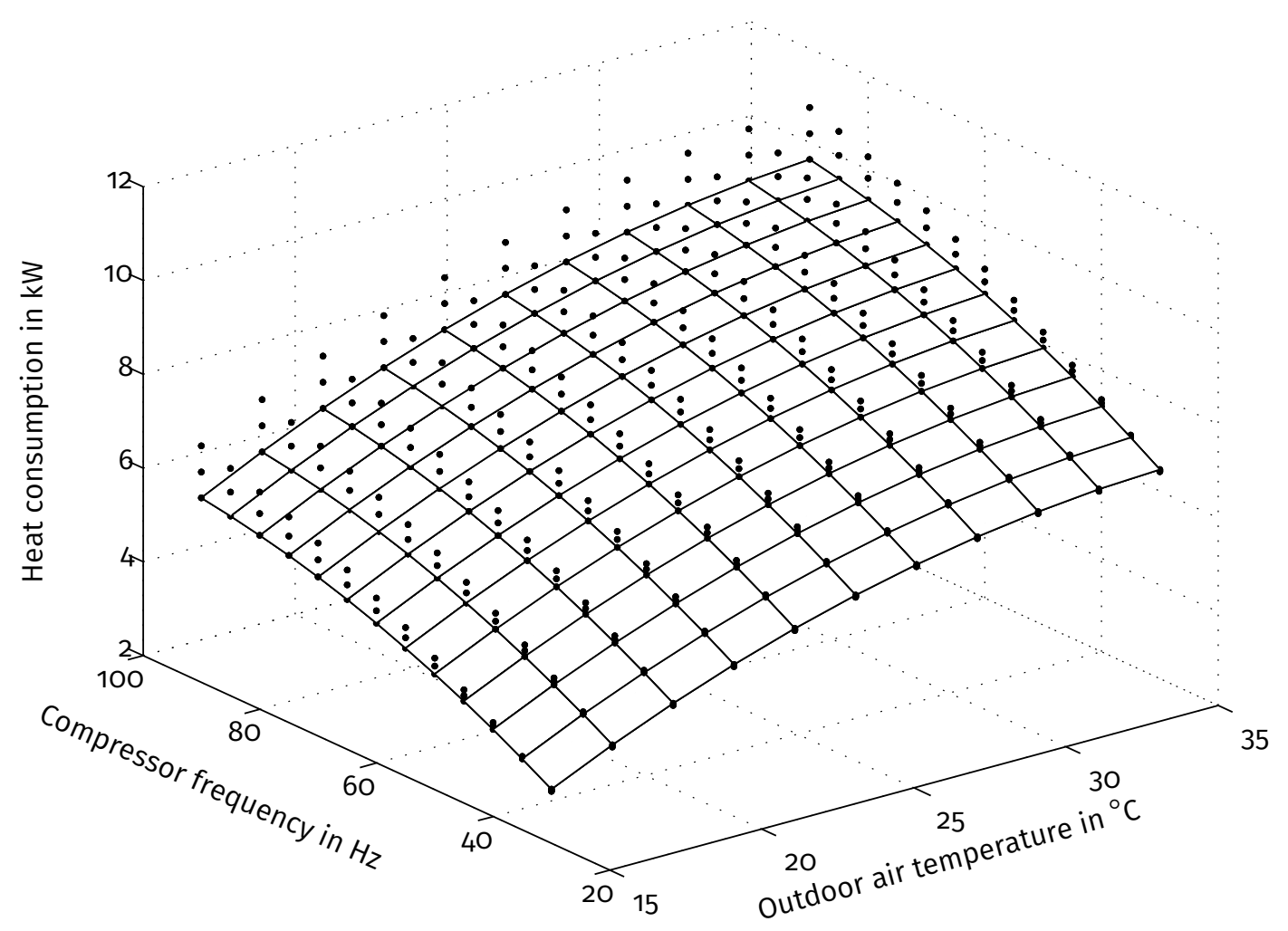

Figure B.19. - Performance Map in Air Conditioning Mode 


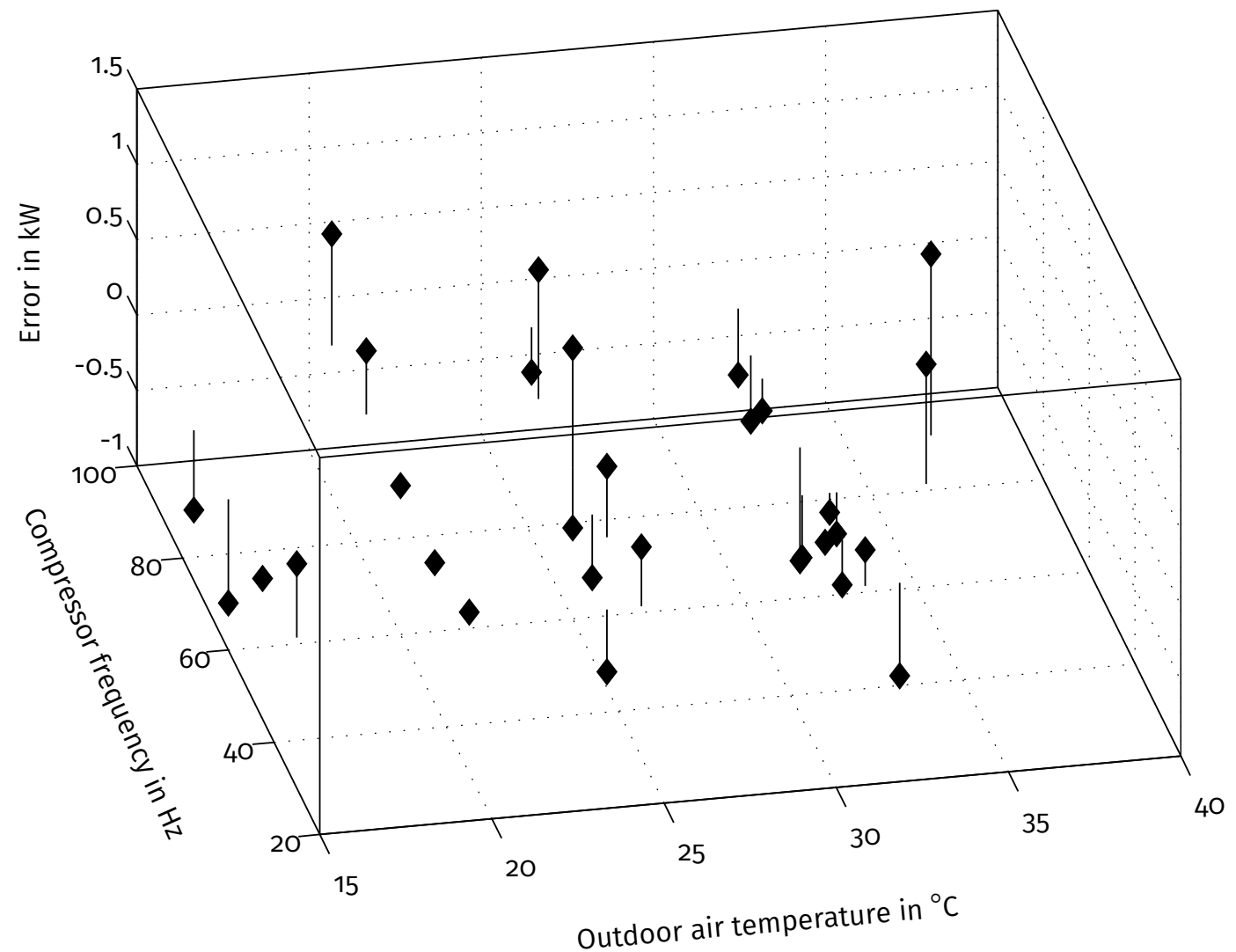

Figure B.20. - Error between AC Performance map and measurement data (Q_AC_est-Q_AC_meas) 
Table B.3. - Measurements for the heat pump performance map

\begin{tabular}{|c|c|c|c|c|c|}
\hline $\begin{array}{r}\text { Temperature } \\
{ }^{\circ} \mathrm{C}\end{array}$ & $\begin{array}{r}\text { rel. Humidity } \\
\%\end{array}$ & $\begin{array}{r}\text { Frequency } \\
\mathrm{Hz}\end{array}$ & $\begin{array}{r}\text { Supply air flow } \\
\mathrm{m}^{3}\end{array}$ & $\begin{array}{r}\text { Heat Performance } \\
\mathrm{kW}\end{array}$ & $\begin{array}{r}\text { Source } \\
-\end{array}$ \\
\hline 9.9 & 44.3 & 60 & 1973 & 13.55 & 1 \\
\hline 10.0 & 41.5 & 75 & 1973 & 14.73 & 1 \\
\hline 10.1 & 40.2 & 80 & 1973 & 15.52 & 1 \\
\hline 9.9 & 39.5 & 85 & 1973 & 15.78 & 1 \\
\hline 10.1 & 38.5 & 90 & 1973 & 15.85 & 1 \\
\hline 10.1 & 46.4 & 50 & 1973 & 12.30 & 1 \\
\hline 10.0 & 41.9 & 50 & 1973 & 10.85 & 1 \\
\hline 9.7 & 44.1 & 40 & 1973 & 10.42 & 1 \\
\hline 9.7 & 44.8 & 35 & 1973 & 9.40 & 1 \\
\hline 9.7 & 45.1 & 30 & 1973 & 8.48 & 1 \\
\hline 10.2 & 42.8 & 50 & 1973 & 11.31 & 1 \\
\hline 7.3 & 46.9 & 90 & 1100 & 11.77 & 1 \\
\hline 15.2 & 35.9 & 80 & 1800 & 12.84 & 1 \\
\hline 15.2 & 35.5 & 90 & 1800 & 14.16 & 1 \\
\hline 12.1 & 48.6 & 30 & 1800 & 7.26 & 1 \\
\hline 11.9 & 48.8 & 40 & 1800 & 8.58 & 1 \\
\hline 12.1 & 46.8 & 50 & 1800 & 9.72 & 1 \\
\hline 12.2 & 45.2 & 60 & 1800 & 11.04 & 1 \\
\hline 12.2 & 44.2 & 70 & 1800 & 11.88 & 1 \\
\hline 12.1 & 43.2 & 80 & 1800 & 12.66 & 1 \\
\hline 12.2 & 41.9 & 90 & 1800 & 13.32 & 1 \\
\hline 9.6 & 53 & 3 & 967 & 6.48 & 1 \\
\hline 10 & 52.5 & 35 & 810 & 5.97 & 1 \\
\hline 10.4 & 51.3 & 35 & 1800 & 8.58 & 1 \\
\hline 3 & 80 & 30 & 1500 & 4.250 & 2 \\
\hline 3 & 80 & 45 & 1500 & 6.2 & 2 \\
\hline 3 & 80 & 60 & 1500 & 6.65 & 2 \\
\hline 3 & 80 & 75 & 1500 & 7.3 & 2 \\
\hline 6 & 80 & 30 & 1500 & 5.05 & 2 \\
\hline 6 & 80 & 45 & 1500 & 7.2 & 2 \\
\hline 6 & 80 & 60 & 1500 & 8.55 & 2 \\
\hline 10 & 80 & 30 & 1500 & 5.8 & 2 \\
\hline 10 & 80 & 45 & 1500 & 8.45 & 2 \\
\hline 15 & 80 & 30 & 1500 & 7.45 & 2 \\
\hline 15 & 80 & 45 & 1500 & 9.65 & 2 \\
\hline
\end{tabular}


Table B.5. - Measurement data for the AC performance map

\begin{tabular}{|c|c|c|c|c|c|}
\hline $\begin{array}{r}\text { Temperature } \\
{ }^{\circ} \mathrm{C}\end{array}$ & $\begin{array}{r}\text { rel. Humidity } \\
\%\end{array}$ & $\begin{array}{r}\text { Frequency } \\
\mathrm{Hz}\end{array}$ & $\begin{array}{r}\text { Supply air flow } \\
\mathrm{m}^{3}\end{array}$ & $\begin{array}{r}\text { Heat Performance } \\
k W\end{array}$ & $\begin{array}{r}\text { Source } \\
-\end{array}$ \\
\hline 31.6 & 41 & 60 & 1100 & 9.57 & 1 \\
\hline 31.5 & 45 & 75 & 1100 & 9.97 & 1 \\
\hline 31.5 & 44.5 & 80 & 1250 & 10.92 & 1 \\
\hline 31.8 & 43 & 90 & 1250 & 11.51 & 1 \\
\hline 31 & 46 & 50 & 1100 & 8.43 & 1 \\
\hline 32 & 43 & 50 & 1250 & 8.54 & 1 \\
\hline 31 & 47 & 40 & 1250 & 7.25 & 1 \\
\hline 31.5 & 46 & 40 & 1250 & 7.58 & 1 \\
\hline 31.5 & 46 & 30 & 1250 & 6.00 & 1 \\
\hline 32.5 & 44 & 30 & 1250 & 6.92 & 1 \\
\hline 31.8 & 47 & 50 & 1250 & 8.38 & 1 \\
\hline 35.4 & 42.5 & 60 & 810 & 7.29 & 1 \\
\hline 34.6 & 47.9 & 50 & 810 & 7.10 & 1 \\
\hline 24.9 & 61.5 & 50 & 810 & 7.32 & 1 \\
\hline 25 & 60.5 & 60 & 810 & 7.86 & 1 \\
\hline 25.8 & 53.4 & 90 & 810 & 8.96 & 1 \\
\hline 24 & 61.5 & 30 & 810 & 5.78 & 1 \\
\hline 15 & 60 & 30 & 1250 & 2.6 & 2 \\
\hline 15 & 60 & 45 & 1250 & 4.6 & 2 \\
\hline 15 & 60 & 60 & 1250 & 6.45 & 2 \\
\hline 15 & 60 & 75 & 1250 & 7.3 & 2 \\
\hline 20 & 60 & 30 & 1250 & 4.4 & 2 \\
\hline 20 & 60 & 45 & 1250 & 6.0 & 2 \\
\hline 20 & 60 & 60 & 1250 & 7.2 & 2 \\
\hline 20 & 60 & 75 & 1250 & 7.8 & 2 \\
\hline 20 & 60 & 90 & 1250 & 8.3 & 2 \\
\hline 25 & 60 & 30 & 1250 & 5.08 & 2 \\
\hline 25 & 60 & 45 & 1250 & 6.5 & 2 \\
\hline 25 & 60 & 60 & 1250 & 7.45 & 2 \\
\hline 25 & 60 & 75 & 1250 & 8.45 & 2 \\
\hline
\end{tabular}




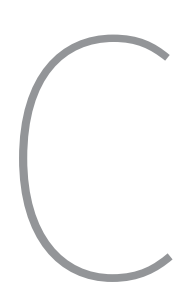

Lists 


\section{List of Acronyms}

CAN Controller Area Network

COA cooling air

COP coefficient of performance

CRM compression refrigeration machine

cS change of state

CWT climatic wind tunnel

EHA exhausted air

EHA Fortluft

EN European Norm

FSM finite state machine

GPS Global Positioning System

HKL Heizungs-, Klima- und Lüftungsanlage

HVAC heating, ventilation and air conditioning system

IDA indoor air

IDA Innenraumluft

KWK Klima-Wind-Kanal

LEA leakage air

LEA Leckageluft

MIA mixing air

MILP mixed integer linear problem

MPC model predictive controller

MVB Multi-function Vehicle Bus

ODA outdoor air

ODA Außenluft

PEM prediction error method

$\phi_{\text {ac }}$ Auxiliary Variable of the air condition 
$\phi_{\text {eh }}$ Auxiliary Variable of the electrical heater

$\phi_{\mathrm{hp}}$ Auxiliary Variable of the heat pump

PMV predicted mean vote

PPD percentage persons dissatisfied

QP quadratic problem

RCA recirculation air

RCA Umluft

RCPP Rapid Controller Prototyping Platform

RTA Rail Tec Arsenal - Fahrzeugversuchsanlage GmbH

SAGOE Siemens AG Österreich

SDPT short-distance public transport

SM state machine

SUP supply air

SUP Zuluft

ULF Ultra Low Floor

VK Vossloh Kiepe

VKA Vossloh Kiepe Austria GmbH

VUT Vienna University of Technology

WP work package 


\section{List of Figures}

1.1. Vehicle energy balance of a driving cycle of the Oslo metro [106, Figure 4.53] . . 1

2.1. Risk-Return-Diagram. 7 and 8 are efficient portfolios. 1,2 and 9 are not efficient [105,

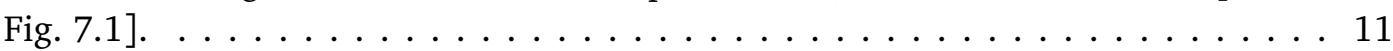

3.1. Closed control loop of the climatisation of the vehicle . . . . . . . . . 17

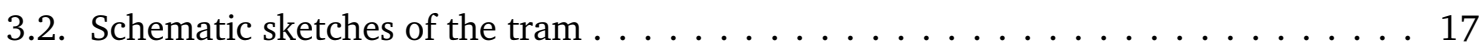

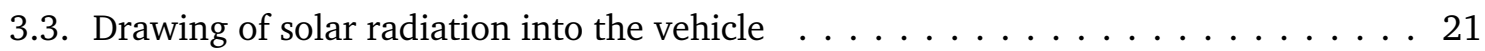

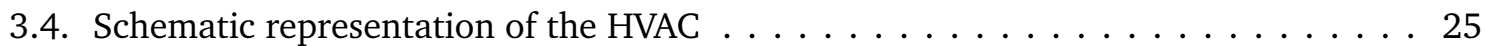

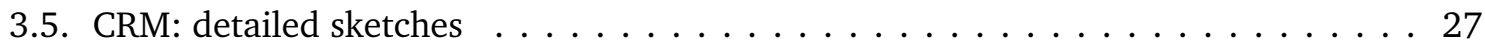

3.6. Schematic diagram of the global FSM of the HVAC $\ldots \ldots \ldots \ldots$

3.7. Schematic diagrams of various finite state machine . . . . . . . . . . . . 31

4.1. Schematic illustration of the second-order thermal vehicle model . . . . . . . . . 36

4.2. Chronological sequence of $x$ and $u$ for special and existing experiments (schematic

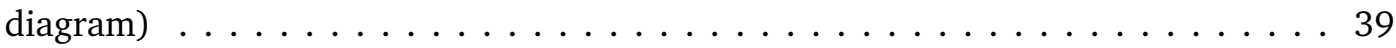

4.3. Occurring indoor air temperature versus outdoor air temperature with original set-point curve and new calculated curve . . . . . . . . . . . . . . . 47

4.4. Number of hours for a given outdoor air temperature . . . . . . . . . . . . . 48

4.5. Indoor air temperature versus outdoor air temperature, measurement points and set point curve . . . . . . . . . . . . . . . . . . . . . . . . 49

4.6. Comparing estimated with original PPD for a $T_{\text {IDA }}$ and $T_{\text {veh }}$ temperature range with

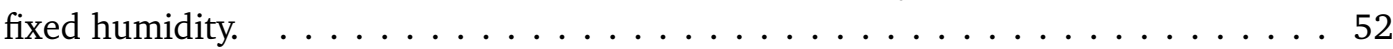

5.1. Current control loop. The current control loop depends on the operational mode. It shows the control while in Heating mode with an operating electric heater. . . . . . 53

5.2. Pareto optimality of the thermal comfort control problem . . . . . . . . . . 55

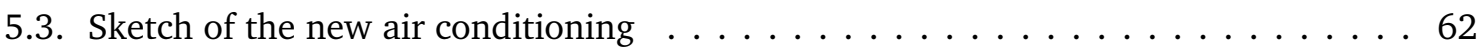

5.4. Schematic diagrams of different parts of the air conditioning system . . . . . 63

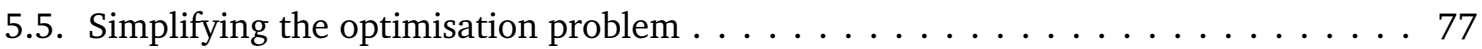

5.6. Both methods for estimating the HVAC mode . . . . . . . . . . . . . . . 83

5.7. Composition of the RCPP and the HVAC controllers on the tram. The figure shows the CAN cable between the RCPP and the HVAC controller as a black dotted line and the power supply of the RCPP as a dashed line. . . . . . . . . . . . . . . 88

6.1. Section 2 - left: indoor air temperature, right: temperature of car body Environmental conditions: $T_{\mathrm{ODA}}=28^{\circ} \mathrm{C}$, sun simulation: $800 \mathrm{~W} / \mathrm{m}^{2}$, passenger simulation: 0 persons Simulation results stay between minimum and maximum measurements. The deviation is small for the temperature of the car body. . . . . . . . . . . . . . 92

6.2. Section 2 - left: indoor air temperature, right: temperature of car body Environmental conditions: $T_{\mathrm{ODA}}: 0^{\circ} \mathrm{C}$ to $28^{\circ} \mathrm{C}$, sun simulation: $600 \mathrm{~W} / \mathrm{m}^{2}$, passenger simulation: 43 persons Simulation results stay between minimum and maximum measurements. The deviation is small for the temperature of the car body. . . . . . . . . . . . . . 93

6.3. left: power consumption of compressor and supply air fan; right: supply air temperature Environmental conditions: $T_{\mathrm{ODA}}: 28^{\circ} \mathrm{C}$, sun simulation: $800 \mathrm{~W} / \mathrm{m}^{2}$, passenger simulation: 0 persons - deviation of power consumption is about $1 \%$, deviation of supply air temperature in measurement accuracy band $\ldots \ldots \ldots \ldots \ldots$ 
6.4. Tram2: Comparison between different models (Simulation) and measurement using data from step responses . . . . . . . . . . . . . . . . . . 95

6.5. Comparison between estimated models (simulation) and measurements for indoor air temperature using data from standard commissioning . . . . . . . . . . 96

6.6. Boxplots of generalised parameters for each vehicle class . . . . . . . . . . . 96

6.7. Number of repetitions for the master controller (measurement file: 260820110447) 98

6.8. Indoor air temperature of the simulated summer day (measurement file: 260820110447) 98

6.9. Comparison for enthalpies of supply air for the master $\left(\Delta h_{\mathrm{SUP}}\right)$ and slave controller

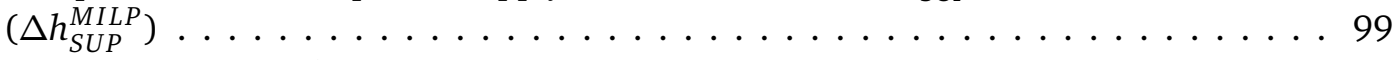

6.10. Full enumeration of the MILP with constant COP . . . . . . . . . . . . . . . 100

6.11.Full enumeration of the MILP with variable COP . . . . . . . . . . . . . . 101

$6.12 .2 \mathrm{D}$ MILP of a winter day . . . . . . . . . . . . . . . . . . . . . 102

$6.13 .2 \mathrm{D}$ MILP of a summer day . . . . . . . . . . . . . . . . . . 103

6.14. Test conditions: $T_{\mathrm{ODA}}=-10^{\circ} \mathrm{C}$, Load simulation: 0 persons, sun simulation: $0 \mathrm{~W} / \mathrm{m}^{2} 106$

6.15. Test conditions: $T_{\mathrm{ODA}}=5^{\circ} \mathrm{C}$, Load simulation: 0 persons, sun simulation: $0 \mathrm{~W} / \mathrm{m}^{2} .107$

6.16. Test conditions of $\mathrm{v} 101 \mathrm{~T}_{\mathrm{ODA}}=20^{\circ} \mathrm{C}$, Load simulation 42 persons, sun simulation

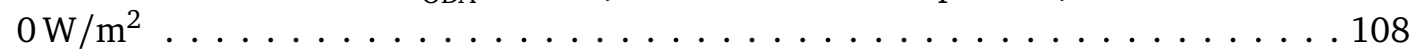

6.17. Test conditions: $T_{\mathrm{ODA}}=28^{\circ} \mathrm{C}$, Load simulation 42 persons, sun simulation $0 \mathrm{~W} / \mathrm{m}^{2} 109$

6.18. Test conditions: $T_{\mathrm{ODA}}=0{ }^{\circ} \mathrm{C}$ to $28^{\circ} \mathrm{C}$, Load simulation 0 persons, sun simulation

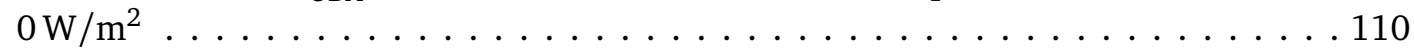

6.19. Test conditions: $T_{\mathrm{ODA}}=0^{\circ} \mathrm{C}$ to $28^{\circ} \mathrm{C}$, Load simulation 0 persons, sun simulation

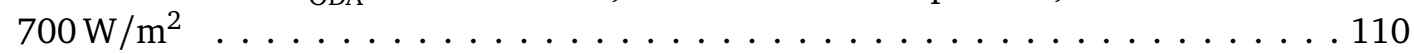

6.20. Comparison of the representative power consumption of HVACs between the current and the new system plotted against the outdoor air temperature . . . . . . . . . 111

6.21. Power saving of the new compared to current system . . . . . . . . . . . . 111

B.1. left: conditions of the outdoor air (temperature and relative humidity), right: solar radiation and rain . . . . . . . . . . . . . . . . . . . . . . 121

B.2. Travelled Route, + Route of the tram (line 62), - travelled route of the tram, • stops 122

B.3. Distance-time graph shows the location of the tram for the test time. Stops are marked as . . . . . . . . . . . . . . . . . . . . . . . 122

B.4. Outdoor air temperature for every section $(1-3)$ of the tram . . . . . . . . 123

B.5. Solar radiation onto the vehicle for every section (1-3) of the tram . . . . . . . 123

B.6. Number of Passengers for every section (1-3) of the tram . . . . . . . . . . . . 124

B.7. Indoor air temperature in every section(1-3) of the tram . . . . . . . . . . . . 124

B.8. Relative humidity of the indoor air for every section(1-3) of the tram . . . . . . . 125

B.9. Thermal comfort given as predicted mean vote . . . . . . . . . . . . . 125

B.10.The percentage shown refers to, with the thermal comfort, dissatisfied passengers. . 126

B.11.Temperature of the supply air for every section(1-3) of the tram . . . . . . . . 126

B.12.Relative humidity of the supply air for every section(1-3) of the tram . . . . . . . 127

B.13.Energy consumption of the $400 \mathrm{~V}$ consumer for every section(1-3) of the tram . . 127

B.14.Energy consumption of the $600 \mathrm{~V}$ consumer for every section(1-3) of the tram . . 128

B.15.Comparison between simulation and measurement: left: $400 \mathrm{~V}$ consumer, right:

$600 \mathrm{~V}$ consumer. . . . . . . . . . . . . . . . . . . . . . . . . 128

B.16.global state of the controller for every section(1-3) of the tram . . . . . . . . . 129

B.17.Performance Map in Heat Pump Mode . . . . . . . . . . . . . . . . . . . . . 131 
B.18.Error between HP performance map and measurement data (Q_HP_est-Q_HP_meas) 132

B.19.Performance Map in Air Conditioning Mode . . . . . . . . . . . . . . . . . 133

B.20.Error between AC Performance map and measurement data (Q_AC_est-Q_AC_meas) 134 


\section{List of Tables}

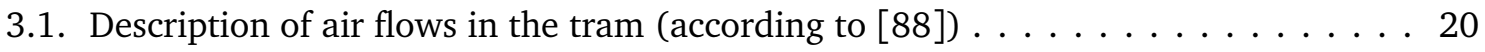

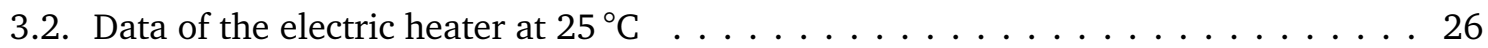

3.3. Volume flow of the supply air fan in $\mathrm{m}^{3} \mathrm{~h}^{-1} \ldots \ldots \ldots \ldots$. . . . . . . . 29

4.1. Overview of the available and evaluated experiments for the estimation of heat capacities and heat transfer coefficient . . . . . . . . . . . . . . 41

4.2. Heat transfer coefficients and heat capacities of existing vehicles . . . . . . . . 43

4.3. Parameter of various set point curves . . . . . . . . . . . . . . . . . 50

4.4. Parameters of the polynomial curve with 4 parameters $\ldots \ldots \ldots \ldots \ldots$

5.1. Number of solutions of the MILP (Complete numbering) . . . . . . . . . . . 82

6.1. Generalised parameters for existing trains . . . . . . . . . . . . . . 97

6.2. Listing of current and new experiments for the calculation of the energy consumption. The mean value of the $0^{\circ} \mathrm{C}$ und $10^{\circ} \mathrm{C}$ experiments is the reference for $5^{\circ} \mathrm{C}$. .105

6.3. Resulting energy consumption of conducted tests. . . . . . . . . . . . 105

A.1. Switching conditions of the global state machine . . . . . . . . . . . . 115

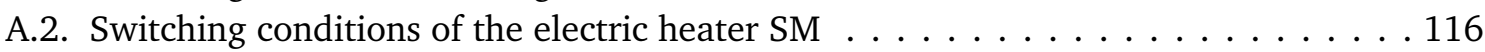

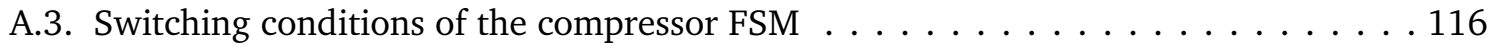

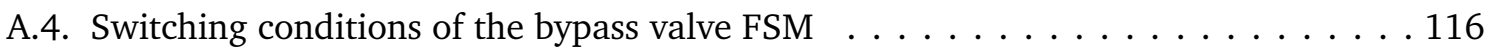

A.5. Switching conditions of the supply air fan FSM . . . . . . . . . . . . 117

A.6. Parameters of the electric heater (Heater $=\operatorname{struct}() ; \ldots \ldots \ldots \ldots \ldots 118$

A.7. Parameters of the cooling cycle (CoolingCycle. = struct ()$; \ldots \ldots \ldots 119$

A.8. Calculated heat transfer coefficient for individual tram parts as a function of the tram speed . . . . . . . . . . . . . . . . . . . . . . . 120

B.1. Factors of the performance maps for the CRM . . . . . . . . . . . . . . . 130

B.3. Measurements for the heat pump performance map . . . . . . . . . . . . . 135

B.5. Measurement data for the AC performance map . . . . . . . . . . . . 136 


\section{List of subscripts and}

\section{superscripts}

AAC After air condition

ac Air condition

air Air

aux Auxiliaries

в Heating (CRM+el)

c Cooling

cH Heating-cooling

CO2 Carbon dioxide

comp Compressor

cond Condensation

cool Cool

D Dehumidification

door Door

E Heating (el)

eh Electrical heater

evap Evaporation

EHA Exhausted air

exheat Exheat

fan Fan

н Heating (CRM)

HC Cooling-heating

hp Heat pump

IDA Indoor air

in In

км Refrigerant corr Correcting factor

light Light

loss Losses from the vehicle to the environment

lb Lower bound of ...

meas Measurement

MILP Mixed-integer linear program

MIA Mixed Indoor air

MPC Model predictive control

oDA Outdoor air

out Out

pas, $\mathrm{CO} 2 \mathrm{CO} 2$ amount of passengers

pas,lat Latent heat of passengers

pas,sen Sensible heat of passengers

pas Passengers

PMv Predicted mean vote

PPD Percentage persons dissatisfied

sol Solar radiation

sec Section

res Residual

s Saturated

sen Sensible

set Set point

sup Supply air

tot Total

ub Upper bound of ...

vapour Vapour

veh Vehicle

v Ventilation

w Water 


\section{List of symbols}

A State (or system) matrix of the state space system

$\alpha$ Ratio of outdoor air

$\alpha$ Flow conditions

$\mathscr{B}$ Heating (el+CRM) mode

B Input matrix of the state space system

$b$ Constants for the constraints

$\mathscr{C}$ Cooling mode

C Output matrix of the state space system

$C$ Heat capacity

c Specific heat capacity

COP Coefficient of performance

$\bar{C}$ Heat capacity total

$\mathscr{D}$ Dehumidification mode

D Feedthrough (or feedforward) matrix of the state space system

$\delta$ Binary control variable.

Usually the volume is further described by an index.

$\Delta u$ Change of the control variable

$\mathscr{E}$ Heating (el) mode

$E$ Energy

$\dot{E}$ Enthalpy flow

$e$ Control error

$\boldsymbol{\varepsilon}$ Prediction error

$f$ Coefficient or scaling factor

$\mathscr{H}$ Heating (CRM) mode

$h$ Enthalpy

$\Delta h_{\text {SUP }}$ The control variable of the MPC problem, effects the temperature

$\Delta h_{\mathrm{v}}$ Evaporation heat of water

$i$ Index

$J$ Objective function

K Noise matrix

$k$ Heat transfer coefficient

$\kappa$ MPC parameter which weights violations of the constraints

$l$ Length

$\lambda$ heat conductivity

$L$ Lower bound

$\mathscr{L}$ Logic

$m$ Mass $\dot{m}$ Mass flow

$n$ Number of ...

$N$ Number of thermal systems

$O$ Surface

$\mathbf{O}_{\text {sol }}$ Power of solar radiation

$\mathscr{P}$ A point in the diagram

$P$ Electric power consumption

$p$ A pressure

$\varphi$ Discrete control variable of the HVAC

$\phi$ Auxiliary variable

$\varphi$ Relative humidity of air

PMV Predicted mean vote

PPD Percentage persons dissatisfied

Q Weighting matrix for the control error

$\dot{Q}$ Heat flow

$Q$ Heat

q Linear Weighting vector of the control error $\dot{q}$ specific Heat flow

$\dot{q}_{\text {sol }}$ Power of solar radiation

$\mathbf{R}$ Weighting matrix for the control vector

$\mathbf{R}^{2}$ Coefficient of determination

$R$ An electrical resistance.

$\rho$ Density

$S$ Entrophy

$\sigma$ Carbon dioxide concentration

SS Sum of squares

$\Delta \sigma_{\text {SUP }}$ The control variable of the MPC problem, effects the $\mathrm{CO} 2$ concentration

$T$ Temperature

$t$ Continuous time

$\tau$ Control horizon

$\boldsymbol{\theta}$ Parameter vector

$\Delta T_{\text {med }}$ Thermodynamic medium temperature

$\mathscr{U}$ Solution candidates

$u$ Linear control variable

$U$ Upper bound

$\mathscr{V}$ Ventilating mode

$U$ Voltage

$V$ A volume

$\dot{V}$ Volume flow

$v$ Vehicle speed

$\dot{W}$ Work by time

$W$ Work

$w$ Reference vector, set-point

$x$ State vector of the state space system 
$\xi$ Angle of solar radiation

$\zeta$ Height angle of solar radiation

$Y$ Absolute humidity of air

$y$ Output vector of the state space system

$\bar{y}$ Means of the output of the model $y$ Output vector of the model

$\hat{y}$ Predicted output of the model

$z$ Control vector of the state space system

$z$ Disturbance vector of the state space system 


\section{Bibliography}

[1] Abdul Afram and Farrokh Janabi-Sharifi. Review of modeling methods for HVAC systems. Applied Thermal Engineering, 67(2):507-19, 2014. ISSN 1359-4311. doi: http://dx.doi. org/10.1016/j.applthermaleng.2014.03.055.

[2] H. Amri, Raphael N. Hofstädter, and M. Kozek. Energy efficient design and simulation of a demand controlled heating and ventilation unit in a metro vehicle. In IEEE Forum on Integrated and Sustainable Transportation System (FISTS), pages 7-12, June 2011. doi: 10.1109/FISTS.2011.5973605.

[3] E. Anderson, Z. Bai, C. Bischof, S. Blackford, J. Demmel, J. Dongarra, J. Du Croz, A. Greenbaum, S. Hammarling, A. McKenney, and D. Sorensen. LAPACK Users' Guide. Society for Industrial and Applied Mathematics, Philadelphia, PA, third edition, 1999. ISBN 0-89871447-8 (paperback).

[4] National Marine Electronics Association. NMEA 0183, The Standard for Interfacing Marine Electronics. Standard NMEA 0183 V 4.00, National Marine Electronics Association, NMEA, 7 Riggs Avenue, Severna Park, MD 21146, 112008.

[5] Hans Dieter Baehr and Stephan Kabelac. Thermodynamik - Grundlagen und technische Anwendungen. Springer, Heidelberg, 14 edition, 2009.

[6] Hans Dieter Baehr and Karl Stephan. Wärme-und Stoffübertragung. Springer DE, 2008.

[7] Reiner Baehr, Hans Dieter und Tillner-Roth. Thermodynamische Eigenschaften umweltverträglicher Kältemittel. Springer, Heidelberg, 1995.

[8] VDI-Gesellschaft Bauen und Gebäudetechnik. Berechnung von Kühllast und Raumtemperaturen von Räumen und Gebäuden (VDI-Kühllastregeln). Norm VDI 2078:2012 Entwurf, Verein Deutscher Ingenieure e.V., Düsseldorf, 032012.

[9] Guillermo Bejarano, Carlos Vivas, Manuel G Ortega, and Manuel Vargas. Suboptimal hierarchical control strategy to improve energy efficiency of vapour-compression refrigeration systems. Applied Thermal Engineering, 125:165-184, 2017.

[10] Thomas Berger and Willibald Böck. Radverkehrserhebung Wien - Entwicklungen, Merkmale und Potenziale, 2010. URL http://www.wien.gv .at/stadtentwicklung/studien/ pdf/b008167.pdf.

[11] Michel Berkelaar, Kjell Eikland, and Peter Notebaert. Open source (Mixed-Integer) Linear Programming system, 2004. URL http://lpsolve. sourceforge.net/5.5/. Multiplatform, pure ANSI C / POSIX source code, Lex/Yacc based parsing; visited on September 4th 2013.

[12] Bart Beusen, Bart Degraeuwe, and Patrick Debeuf. Energy savings in light rail through the optimization of heating and ventilation. Transportation Research Part D: Transport and Environment, 23(0):50-4, 2013. ISSN 1361-9209. doi: http://dx.doi.org/10.1016/j.trd. 2013.03.005. 
[13] Thilo Blasse. Rail vehicle with an air-permeable wall for internal ventilation by displacement or source ventilation, 05 2015. EP 2868502.

[14] Florin Bode, Ilinca Nastase, Paul Danca, and Amina Meslem. The influence of the inlet angle of vehicle air diffuser on the thermal comfort of passengers. In ENERGY and ENVIRONMENT (CIEM), 2017 International Conference on, pages 442-446. IEEE, 2017.

[15] Anneliese Böttiger. Regelungstechnik: Eine Einführung für Ingenieure und Naturwissenschaftler. Oldenbourg, 1991.

[16] Romain Bourdais and Hervé Guéguen. Distributed predictive control for complex hybrid system. the refrigeration system example. IFAC Proceedings Volumes, 43(8):388-394, 2010.

[17] Stefan P. Boyd and L. Vandenberghe. Convex optimization. Cambridge Univ Pr, 2004.

[18] J. Steven Brown and Piotr A. Domanski. Review of alternative cooling technologies. Applied Thermal Engineering, 64(2):252-62, 2014. ISSN 1359-4311. doi: http://dx.doi.org/10. 1016/j.applthermaleng.2013.12.014.

[19] Michael Canori. Anzahl der installierten lampen. mündliche Mitteilung, 2010.

[20] Charles M. Close and Dean K. Frederick. Modeling and Analysis of Dynamic Systems. John Wiley \& Sons, Inc., second edition, 1994. ISBN 0471125172.

[21] J. Dahl and L. Vandenberghe. Cvxopt: A python package for convex optimization, 2005. URL http: //www. ee.ucla.edu/ vandenbe/cvxopt.

[22] Danfoss. statische Verdichterkennlinie des MTZ64-4. zur Verfügung gestellt durch VosslohKiepe, 2008.

[23] DIN EN 13129-2:2004-10. Railway applications - Air conditioning for main line rolling stock - Part 2: Type tests, 10 2004. German Version.

[24] DIN EN 14750-2:2006-08. Railway applications - Air conditioning for urban and suburban rolling stock - Part 2: Type tests, 08 2006. German Version.

[25] DIN V 18599. Energy efficiency of buildings - Calculation of the net, final and primary energy demand for heating, cooling, ventilation, domestic hot water and lighting, 2011. Prestandard, German Version.

[26] dSPACE GmbH. Hardware Installation and Configuration, 2011. Kapitel /chapter Data Sheet Micro AutoBox II 1401/1511.

[27] dSPACE GmbH. Receiving GPS Data with the MicroAutoBox, 2013. URL http://www.dspace.com/en/ltd/home/support/kb/supapnot/mabxappnote/ appnotegps .cfm. [Online; 2013-10-30].

[28] Christian Dullinger, Walter Struckl, and Martin Kozek. A modular thermal simulation tool for computing energy consumption of hvac units in rail vehicles. Applied Thermal Engineering, 78:616-629, 2015. 
[29] Projektkonsortium EcoTram. Förderantrag: Energieoptimierung der thermischen Fahrzeugsysteme bei Schienenfahrzeugen. Formularteil A, 2009.

[30] Projektkonsortium EcoTram. Förderantrag: Energieoptimierung der thermischen Fahrzeugsysteme bei Schienenfahrzeugen - Evaluierung. Formularteil A, 2011.

[31] Matthew S Elliott and Bryan P Rasmussen. Model-based predictive control of a multievaporator vapor compression cooling cycle. In American Control Conference, 2008, pages 1463-1468. IEEE, 2008.

[32] Nikolaus Euler-Rolle. Blackbox Modellierungen im Rahmen des Projekts EcoTram. Projektarbeit, 2011.

[33] European Comission. A sustainable future for transport - towards an integrated, technologyled and user-friendly system, 2009. URL http://ec.europa.eu/transport/ strategies/2009_future_of_transport_en.htm. pp. 26.

[34] European Commission. EUROPE 2020: A European strategy for smart, sustainable and inclusive growth. Publications Office of the European Union, Brussels, 3 2010. EN Version.

[35] European Parliament and the Council. Directive 2010/31/EU of the European Parliament and of the Council of 19 May 2010 on the energy performance of buildings (recast), 2010.

[36] B.S. Everitt. Cambridge Dictionary of Statistics. Cambridge University Press, 1998. ISBN 9780521593465.

[37] Felix Felgner, Rolf Merz, and Lothar Litz. Modular modelling of thermal building behaviour using modelica. Mathematical and Computer Modelling of Dynamical Systems, 12(1):35-49, 2006.

[38] Carlos E Garcia, David M Prett, and Manfred Morari. Model predictive control: theory and practice-a survey. Automatica, 25(3):335-348, 1989.

[39] Anton Glaninger. Skriptum zur Vorlesung: Lüftung und Klima. Sommersemester 2009, 2009.

[40] GlobalSat Technology Corporation. Product User Manual: RS232 GPS Receiver BR-355 GlobalSat, 2013. URL http://www.globalsat.com.tw/globalsat_admin/new_file_ download . php?Pact=FileDownLoad\&Pval=943. [Online; 2013-10-30].

[41] Jörg Grote, Karl-Heinrich ; Feldhusen. Dubbel; Taschenbuch für den Maschinenbau. Springer Berlin Heidelberg: Berlin, Heidelberg, Berlin, Heidelberg, 2011. ISBN 978-3-642-17305-9.

[42] L. Grüne, S. Sager, F. Allgöwer, H. G. Bock, and M. Diehl. Vorausschauend planen, gezielt handeln - über die Regelung und Steuerung technischer Prozesse. In V. Mehrmann M. Grötschel, K. Lucas, editor, Produktionsfaktor Mathematik, pages 27 - 62. Springer Verlag, 2009. URL http://num.math.uni-bayreuth.de/de/publications/2009/gruene_et_ al_prod_faktor_math_2009/gruene_et_al_prod_faktor_math_2009.pdf. DOI: 10.1007/978-3-540-89435-3. 
[43] Inc. Gurobi Optimization. Gurobi optimizer reference manual, 2014. URL http://www . gurobi.com.

[44] Gabriel Haller. Der neue klima-wind-kanal in wien. In ZEVrail Glasers Annalen 126, 38. Grazer Schienenfahrzeugtagung, pages 22-7. Institute of Railway Engineering and Transport Economy, Graz University of Technology, 2002.

[45] Gabriel Haller. The Benefits of Climatic Testing of Rail Vehicles. Foreign Rolling Stock, 3:11, 2009.

[46] Hinrich Helms and Udo Lambrecht. The potential contribution of light weighting to reduce transport energy consumption. The International Journal of Life Cycle Assessment, 12(1): 58-64, 2007.

[47] Klaus Hiller, Emanuel Bunger, and Erwin Firschau. New locomotives for shunting and line operations. Rail Technology Review, 52:24-28, 09 2012. ISSN 1869-7801.

[48] Raphael Hofstädter and Martin Kozek. Deliverable zum AP 1.5.1: Spezifikationen (des Reglers) festlegen. Projektunterlagen EcoTram, 2010.

[49] Raphael Hofstädter and Martin Kozek. Deliverable zum AP 1.5.2 und 1.5.3: Beschreibung des gesamten Simulationsmodells. Projektunterlagen EcoTram, 2011.

[50] Raphael Hofstädter and Martin Kozek. Simulationsberichte eines Tages. Projektunterlagen EcoTram, 2011.

[51] Raphael Hofstädter and Martin Kozek. Holistic Thermal Simulation Model of a Tram. In Proceedings of the 2nd International Conference on Computer Modelling and SimulationCSSim2011, 2011. ISBN 9788021443204. Talk: CSSim2011, 2nd International Conference on Computer Modelling and Simulation, Brno, Czech Republic; 2011-09-05 - 2011-09-07.

[52] Raphael Hofstädter and Martin Kozek. Extension of a Thermal Simulation Model for a Tram and Simulation with On-Site Measurement Data. In Preprints Mathmod 2012 Vienna, Abstract Volume, page 78, 2012. URL http://publik.tuwien.ac.at/files/ PubDat_206933.pdf. Talk: MATHMOD 2012 - 7th Vienna International Conference on Mathematical Modelling, Vienna; 2012-02-14 - 2012-02-17.

[53] Raphael Hofstädter and Martin Kozek. Thermal Vehicle Model Report (Part of Deliverable d 2.1). Project documents OSIRIS, 2013.

[54] Raphael Hofstädter, Martin Kozek, and Gregor Richter. Modular thermodynamic modelling of a light rail vehicle. In Book of extendes abstracts, pages 100-102, 2010. ISBN 978-9491085-02-4. Talk: Railways and Environment, Delft; 2010-12-16 - 2010-12-17.

[55] Raphael N. Hofstädter and Martin Kozek. Spezifikation des Reglers festlegen. Deliverable im Projekt EcoTram zum Arbeitspaket 2.3.1, 2012.

[56] Raphael N Hofstädter, Thomas Zero, Christian Dullinger, Gregor Richter, and Martin Kozek. Heat capacity and heat transfer coefficient estimation for a dynamic thermal model of rail vehicles. Mathematical and Computer Modelling of Dynamical Systems, pages 1-14, 2016. 
[57] Raphael N. Hofstadter, Jorge Amaya, and Martin Kozek. Energy optimal control of thermal comfort in trams. Applied Thermal Engineering, 143:812 - 821, 2018. ISSN 1359-4311. doi: https://doi.org/10.1016/j.applthermaleng.2018.07.084. URL http: //www.sciencedirect.com/science/article/pii/S1359431117378201.

[58] Tobias Gybel Hovgaard, Lars FS Larsen, Kristian Edlund, and John Bagterp Jørgensen. Model predictive control technologies for efficient and flexible power consumption in refrigeration systems. Energy, 44(1):105-116, 2012.

[59] Normenausschluss Schienenfahrzeuge im DIN. Bahnanwendungen - Luftbehandlung in Schienenfahrzeugen des Fernverkehrs - Teil 2: Typprüfungen. Norm DIN EN 13129-2:2004, DIN Deutsches Institut für Normung e.V., Berlin, 102004.

[60] Normenausschluss Schienenfahrzeuge im DIN. Bahnanwendung - Luftbehandlung in Schienenfahrzeugen des innerstädtischen und regionalen Nahverkehrs - Teil 1 Behaglichkeitsparameter. Norm DIN EN 14750-1:2006, DIN Deutsches Institut für Normung e.V., Berlin, 082006.

[61] Normenausschuss Ergonomie im DIN. Ergonomie der thermischen Umgebung - Analytische Bestimmung und Interpretation der thermischen Behaglichkeit durch Berechnung des PMVund des PPD-Indexes und Kriterien der lokalen thermischen Behaglichkeit. Norm DIN EN ISO 7730:2005, DIN Deutsches Institut für Normung e.V., Berlin, 052006.

[62] Intergovernmental Panel on Climate Change. Climate Change: The IPCC scientific assessment. Cambridge University Press, Cambridge, Great Britain, 1990. ISBN 0-521-40720-6. ca. 414 pages.

[63] Christoph Isenschmid, Stefan Menth, and Peter Oelhafen. Energieverbrauch und Einsparpotential des S-Bahn-Gliederzugs RABe 525 "Nina" der BLS AG. Eisenbahn-Revue, 8-9:398-403, 2013.

[64] Lars Johannesson, Mattias Asbogard, and Bo Egardt. Assessing the potential of predictive control for hybrid vehicle powertrains using stochastic dynamic programming. IEEE Transactions on Intelligent Transportation Systems, 8(1):71-83, 2007.

[65] J. Kallrath. Gemischt-ganzzahlige Optimierung: Modellierung in der Praxis: mit Fallstudien aus Chemie, Energiewirtschaft, Metallgewerbe, Produktion und Logistik. Vieweg+ Teubner, Braunschweig u.a., 2002.

[66] J. Kennedy and R. Eberhart. Particle swarm optimization. In IEEE International Conference on Neural Networks, 1995. Proceedings, volume 4, pages 1942-8, Nov 1995. doi: 10.1109/ ICNN.1995.488968.

[67] Fabian Kennel, Daniel Gorges, and Steven Liu. Energy management for smart grids with electric vehicles based on hierarchical mpc. IEEE Transactions on industrial informatics, 9 (3):1528-1537, 2013.

[68] Michaela Killian, Barbara Mayer, and Martin Kozek. Hierarchical fuzzy mpc concept for building heating control. IFAC Proceedings Volumes, 47(3):12048-12055, 2014. 
[69] Joyce Kim, Stefano Schiavon, and Gail Brager. Personal comfort models-a new paradigm in thermal comfort for occupant-centric environmental control. Building and Environment, 2018.

[70] Wolfram Knörr, Christoph Heidt, and Alexander Schacht. Aktualisierung „Daten- und Rechenmodell: Energieverbrauch und Schadstoffemissionen des motorisierten Verkehrs in Deutschland 1960-2030“ (TREMOD) für die Emissionsberichterstattung 2013 (Berichtsperiode 1990-2011). Endbericht, FKZ-No. 36016 037, Umweltbundesamt, Postfach 1406, 06813 Dessau, 092012.

[71] Yassine Koubaa. Application of least-squares techniques for induction motor parameters estimation. Mathematical and Computer Modelling of Dynamical Systems, 12(4):363-75, 2006.

[72] Martin Kozek and Raphael N. Hofstädter. Model predictive control for hvac in light rail and metro vehicles. In International Workshop: Urban and Suburban Rail Vehicles - New Challenges for Climatic Testing. Vienna Climatic Wind Tunnel, 2012.

[73] Daniel Kristanto and Thananchai Leephakpreeda. Energy conversion for thermal comfort and air quality within car cabin. In IOP Conference Series: Materials Science and Engineering, volume 187, page 012037. IOP Publishing, 2017.

[74] Lars FS Larsen, Tobias Geyer, and Manfred Morari. Hybrid model predictive control in supermarket refrigeration systems. IFAC Proceedings Volumes, 38(1):313-318, 2005.

[75] Bin Li, Richard Otten, Vikas Chandan, William F Mohs, Jeff Berge, and Andrew G Alleyne. Optimal on-off control of refrigerated transport systems. Control Engineering Practice, 18 (12):1406-1417, 2010.

[76] Wenhua Li and Jian Sun. Numerical simulation and analysis of transport air conditioning system integrated with passenger compartment. Applied Thermal Engineering, 50(1):37-45, 2013. ISSN 1359-4311. doi: http://dx.doi.org/10.1016/j.applthermaleng.2012.05.030.

[77] Weiwei Liu, Qihong Deng, Wenjie Huang, and Rui Liu. Variation in cooling load of a moving air-conditioned train compartment under the effects of ambient conditions and body thermal storage. Applied Thermal Engineering, 31(7):1150-62, 2011. ISSN 1359-4311. doi: http://dx.doi.org/10.1016/j.applthermaleng.2010.12.010.

[78] L. Ljung. System Identification: Theory for the User. Pearson Education, 1998. ISBN 9780132440530 .

[79] Lennart Ljung. Prediction error estimation methods. Circuits, Systems and Signal Processing, 21(1):11-21, 2002. ISSN 0278-081X. doi: 10.1007/BF01211648.

[80] J. Löfberg. Yalmip : A toolbox for modeling and optimization in MATLAB. In Proceedings of the CACSD Conference, Taipei, Taiwan, 2004. URL http://users.isy.liu.se/johanl/ yalmip. 
[81] Uwe Maaßen. Energieflussbild 2015 für die Bundesrepublik Deutschland in PJ. Technical report, AG Energiebilanzen e.V., Berlin Charlottenburg, 07 2016. URL www. ag-energiebilanzen.de. (German).

[82] MAGNA. Kuli: Thermal management simulation software, 06 2014. URL http://www . kuli.at.

[83] Jacques Marachlian, Riad Benelmir, Amin El Bakkali, and Gérard Olivier. Exergy based simulation model for vehicle HVAC operation. Applied Thermal Engineering, 31(5):696-700, 2011. ISSN 1359-4311. doi: http://dx.doi.org/10.1016/j.applthermaleng.2010.10.001. URL http://www. sciencedirect.com/science/article/pii/S135943111000428X.

[84] David Marcos, Francisco J. Pino, Carlos Bordons, and Jos é J. Guerra. The development and validation of a thermal model for the cabin of a vehicle. Applied Thermal Engineering, 66(2):646-56, 2014. ISSN 1359-4311. doi: http://dx.doi.org/10.1016/j.applthermaleng. 2014.02.054.

[85] MATLAB. version 7.10.0 (R2010a). The MathWorks Inc., Natick, Massachusetts, 2010.

[86] Barbara Mayer, Michaela Killian, and Martin Kozek. A branch and bound approach for building cooling supply control with hybrid model predictive control. Energy and Buildings, 128: 553-566, 2016. ISSN 0378-7788. doi: http://doi.org/10.1016/j.enbuild.2016.07.027. URL http://www.sciencedirect.com/science/article/pii/S0378778816306235.

[87] Rawaida Muhammad, Muhammad Khiril Kamaruddin, and Yeoh Poh See. Influence of passenger car air conditioner system thermostat level setting to fuel consumption and thermal comfort. In Engineering Applications for New Materials and Technologies, pages 183-195. Springer, 2018.

[88] Normenausschluss Heiz- und Raumlufttechnik im DIN. Lüftung von Nichtwohngebäuden - Allgemeine Grundlagen und Anforderungen für Lüftungs- und Klimaanlagen und Raumkuehlsysteme. Norm DIN EN 13779, DIN Deutsches Institut für Normung e.V., Berlin, 092007.

[89] Normenausschluss im DIN. Glas im Bauwesen - Bestimmung der lichttechnischen und strahlungsphysikalischen Kenngrößen von Verglasungen. Technical Report DIN EN 410, DIN Deutsches Institut für Normung e.V., Berlin, 2011.

[90] Normenausschuss Chemischer Apparatebau im DIN. Fließschemata für verfahrenstechnische Anlagen - Allgemeine Regeln. Norm DIN EN 13779, DIN Deutsches Institut für Normung e.V., Berlin, 032001.

[91] Katsuhiko Ogata. Discrete-Time Control Systems (2nd Edition). Prentice Hall, 2 edition, 1 1995. ISBN 9780130342812.

[92] OpenstreetMap. Die freie Wiki-Weltkarte, 2011. URL http://www.openstreetmap. org/. last visited on 2011-08-30. 
[93] J.P. Powell, A. González-Gil, and R. Palacin. Experimental assessment of the energy consumption of urban rail vehicles during stabling hours: Influence of ambient temperature. Applied Thermal Engineering, 66(2):541-7, 2014. ISSN 1359-4311. doi: http://dx.doi.org/10.1016/j.applthermaleng.2014.02.057.

[94] Hermann Recknagel, Eberhard Sprenger, and Ernst-Rudolf Schramek. Taschenbuch für Heizung + Klimatechnik. Oldenbourg Industrieverlag, 2007.

[95] Gabriel Richter, Gregor und Haller. Energieverbrauchsmessungen an einer Straßenbahn Typ A1 Ulf im Rahmen des Forschungsprojekt EcoTram. RTA Rail Tec Arsenal Fahrzeugversuchsanlage GmbH, Wien, 2 edition, 11 2010. Zahl 825443.

[96] Gabriel Richter, Gregor und Haller. Energieverbrauchsmessungen an einer Straßenbahn Typ A1 Ulf im Rahmen des Forschungsprojekt EcoTram II. RTA Rail Tec Arsenal Fahrzeugversuchsanlage GmbH, Wien, 11 2013. Zahl 829788.

[97] Gregor Richter. Ecotram research project: How much energy is used by the HVAC of a tram? Measurements in the climatic wind tunnel and in service. Railvolution, 10(6), 2010.

[98] Nadja Richter. Daten zum Verkehr - Ausgabe 2012. Umweltbundesamt, Postfach 1406, 06813 Dessau, 10 2012. URL www. umweltbundesamt.de. (German).

[99] Michael Rosenberger, Birgit Akagündüz-Binder, Katharina Conrad, Mathis Falter, Rainer Hauswirth, Barbara Hetzmannseder, Josef Hutter, Martina Liebhart, Kurt Mittringer, Müllner Kirsten, Beatrix Rauscher, and Katharina Söpper. STEP 2025 - Urban Development Plan Vienna. Vienna City Administration, Municipal Department 18 (MA 18) - Urban Development and Planning, June 2014. URL https://www . wien.gv . at/stadtentwicklung/ studien/pdf/b008379b.pdf. 145 pages.

[100] J.P. Rugh, K. Bennion, A. Brooker, J. Langewisch, K. Smith, and J. Meyer. PHEV/EV integrated vehicle thermal management - development of a KULI model to assess combined cooling loops. In Institution of Mechanical Engineers (IMechE), editor, Vehicle Thermal Management Systems Conference and Exhibition (VTMS10), pages 649-660. Woodhead Publishing, 2011. ISBN 978-0-85709-172-7. doi: http://dx.doi.org/10.1533/ 9780857095053.8.649. URL http://www. sciencedirect.com/science/article/ pii/B9780857091727500567.

[101] Klaus Gerhard Schmidt. M1 Wärmeübergang an berippten Rohren. In VDI-Wärmeatlas, VDI-Buch, pages 1459-1465. Springer Berlin Heidelberg, 2013. ISBN 978-3-642-19980-6.

[102] Wilhelm Schneider, Stefan Haas, and Karl Ponweiser. Repetitorium Thermodynamik. Oldenbourg Verlag, 2012.

[103] Petra Seibert. Aktuelle meteorologische Messwerte, 12 2010. URL http://www . boku . ac . at/met/wetter/aktuell/. last viseted on 2011-09-01.

[104] ELMOS Semiconductor AG. Integrated Solar Angle Sensor (2 axis), E910.86 Evaluation Kit, 2012. Application Note. 
[105] Klaus Spremann. Portfoliomanagement. Oldenbourg Verlag, 2006.

[106] Walter Struckl. Green Line - Umweltgerechte Produktentwicklungsstrategien für Schienenfahrzeuge auf Basis der Lebenszyklusanalyse des Metrofahrzeuges Oslo. PhD thesis, Vienna University of Technology, 2007.

[107] Inc. The MathWorks. Embedded MATLAB Users' Guide. 3 Apple Hill Drive, Natick, MA 01760-2098, 2007.

[108] Bárbara Torregrosa-Jaime, Filip Bjurling, José M Corberán, Fausto Di Sciullo, and Jorge Payá. Transient thermal model of a vehicle's cabin validated under variable ambient conditions. Applied Thermal Engineering, 2014.

[109] Heinz Unbehauen. Regelungstechnik I - Klassische Verfahren zur Analyse und Synthese linearer kontinuierlicher Regelsysteme, Fuzzy-Regelsysteme. Vieweg, Braunschweig, 1982.

[110] Heinz Unbehauen. Regelungstechnik II - Zustandsregelung, digitale und nichtlineare Regelsysteme. Vieweg, Braunschweig/Wiesbaden, 1983.

[111] Normenausschuss Heiz und Raumlufttechnik. Heizungsanlagen in Gebäuden - Verfahren zur Berechnung der Norm-Heizlast. Norm DIN EN 12831:2003, DIN Deutsches Institut für Normung e.V., Berlin, 082003.

[112] Michael Canori und Walter Struckl. Katalog möglicher Umbaumaßnahmen erstellen. Deliverable zum Arbeitspaket 1.6.3, 2011.

[113] Peter JM Van Laarhoven and Emile HL Aarts. Simulated annealing. Springer, 1987.

[114] Aditya Velivelli, Daniel Guerithault, and Stefan Stöwe. Optimum seat cooling distribution for targeted human thermal comfort ${ }^{\circledR}$. SAE International Journal of Passenger Cars-Mechanical Systems, 10(2017-01-0170):128-134, 2017.

[115] Verordnung zur Änderung der Energieeinsparverordnung. Bundesgesetzblatt Jahrgang 2013 Teil I Nr. 67. Bundesanzeiger Verlag, pages 3951-90, 2013.

[116] Peter Von Böckh and Thomas Wetzel. Wärmeübertragung: Grundlagen und Praxis. Springer DE, 2011.

[117] Vossloh-Kiepe. Konstruktionszeichnung des Heizregisters. Zeichnungsnummer 98.290 338.001, 2008.

[118] Vossloh-Kiepe. Kiepe heißgasbypass. Ergebnisse 05.08.2011, 2011.

[119] Yang Wang and Stephen Boyd. Fast model predictive control using online optimization. Control Systems Technology, IEEE Transactions on, 18(2):267-278, 2010.

[120] Wiener Linien GmbH \& Co KG. 2014 - Facts and Figures. Vienna, 2014. URL https : / www . wienerlinien.at/media/files/2015/facts_and_figures_2014_151139.pdf. 
[121] Wikipedia. Kaskadenregelung - Wikipedia, Die freie Enzyklopädie, 2012. URL http: // de.wikipedia.org/w/index.php?title=Kaskadenregelung\&oldid=102912995. [Online; 2013-10-02].

[122] M. Zeller and U. Busweiler. VDI-Wärmeatlas, chapter Mk - Be- und Entfeuchten von Luft. Springer, Heidelberg, 10 edition, 2006.

[123] Tengfei Zhang and Qingyan (Yan) Chen. Novel air distribution systems for commercial aircraft cabins. Building and Environment, 42(4):1675 - 1684, 2007. ISSN 0360-1323. doi: http://dx.doi.org/10.1016/j.buildenv.2006.02.014. URL http://www . sciencedirect . com/science/article/pii/S0360132306000618.

[124] Zhenying Zhang, Shengjun Liu, and Lili Tian. Thermodynamic analysis of air cycle refrigeration system for chinese train air conditioning. Systems Engineering Procedia, 1(0):16-22, 2011. ISSN 2211-3819. doi: http://dx.doi.org/10.1016/j.sepro.2011.08.004. Engineering and Risk Management. 


\section{Curriculum vitae}

\section{Persönliche Daten}

\author{
Titel Dipl.-Ing. \\ Name Raphael Hofstädter \\ Anschrift Am Berg 38, \\ D-38229 Salzgitter, Deutschland \\ Telefon +4917678942591 \\ E-Mail r.hofstaedter@gmail.com
}

\author{
Geburtsdaten 22. März 1985 in Wien \\ Staatsbürgerschaft Österreich \\ Präsenzdienst abgeleistet, \\ in den Reservestand beordert \\ Familienstand verheiratet \\ Führerschein $A, B$
}

\section{Schulbildung und Studium}

Schulbildung

09/1995-06/1999

$09 / 1999-06 / 2004$

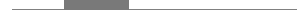

$10 / 2004-06 / 2005$

$10 / 2005-12 / 2009$

Abschlusszeugnis, Bundesgymnasium und Realgymnasium, Mödling.

Matura, Höhere Bundes-, Lehr- und Versuchanstalt für chemische Industrie, Wien, Biochemie, Bio- und Gentechnologie.

Ingenieurarbeit: Enantioselektive Bayer Villiger Oxidation von prochiralen Cyclobutanonen mit genetisch veränderten $\mathrm{E}$. coli

\section{Wehrdienst}

Grundwehrdiener, Österreichs Bundesheer, Allentsteig, Panzerartilleriebataillon 3.

Tätigkeit: Rechengehilfe der Artillerie, Dienstgrad: Korporal der Miliz

Freiwillige Meldung zur Ableistung von Kaderübungen und des Milizunteroffizierskurs 1

Ableistung einer Kaderübung 2012, in den Reservestand beordert 2014

\section{Studium}

Dipl.-Ing., Technische Universität Wien, Wien, Verfahrenstechnik (Apparate-, Anlagen- und Prozesstechnik).

Schwerpunkte: Umwelt und Ressourcenmanagement, Energietechnik

Diplomarbeit: Grundlage für das Regelungskonzept einer Ozonungsstufe für kommunales Abwasser

03/2010 - 11/2018 Dr. techn., Technische Universität Wien, Wien, Institut für Mechanik u. Mechatronik, Abteilung für Regelungstechnik u. Prozessautomatisierung.

Doktorarbeit: Energy optimal control of thermal comfort in trams

\section{Berufserfahrung und Praktika}

\section{Tätigkeit während des Diplomstudiums}

$12 / 2006-3 / 2008$

Chemotechniker - 10h, GWC Water Consultant GmbH, Wien, Analytisches Labor. Tätigkeit: Analyse von Kesselspeise- und Kühlwasserproben

SS 08 tSS 09 Tutor - 5h, Technische Universität Wien, Wien, Institut für Mechanik u. Mechatronik. Tätigkeit: Unterstützung der Lehre in der Laborübung Mess- und Schwingungstechnik 


\section{Berufserfahrung}

03/2010 - 12/2013 Projektassistent - 40h, Technische Universität Wien, Wien, Institut für Mechanik u. Mechatronik, Abteilung für Regelungstechnik u. Prozessautomatisierung.

Tätigkeit: Wissentschaftlicher Mitarbeiter in (inter)nationalen Forschungsprojekten

$01 / 2014-$ jetzt

Patent- und Innovationsmanager - 39h, Alstom Transport Deutschland GmbH, Salzgitter, Engineering, Method \& Tools.

Innovationsmanagement am Standort Salzgitter

Patentmanagement am Standort Salzgitter

\section{Sprachen}

Deutsch Muttersprache

Englisch Verstehen C1, Sprechen B2, Schreiben C1

Europäische Kompetenzstufe

\section{IT Kompetenzen}

Büroprogramme MS Office (Word, Excel, PowerPoint, Visio, Project), LibreOffice

Berechnungssoft. Matlab und Simulink, Maple

CAD AutoCAD (Grundkenntnisse)

Programmieren Python, PHP, $\mathrm{C} / \mathrm{C}++$

Weiteres ATTEX$_{\mathrm{E}} \mathrm{X}, \mathrm{HTML}, \mathrm{CSS}$

Salzgitter, 3. Oktober 2018 